\title{
Measurement and Interpretation of Moments of the Combined Hadronic Mass and Energy Spectrum in Inclusive Semileptonic B-Meson Decays
}

DISSERTATION

zur Erlangung des akademischen Grades

Doctor rerum naturalium

(Dr. rer. nat.)

vorgelegt

der Fakultät Mathematik und Naturwissenschaften

der Technischen Universität Dresden

von

Diplom-Physikerin Verena Klose geboren am 17. Juli 1979 in Hannover 
Gutachter : Prof. Dr. Klaus R. Schubert Prof. Dr. Heiko Lacker Prof. Dr. Thomas Mannel 


\section{Abstract}

This thesis presents first measurements of moments of the hadronic $n_{X}^{2}$ distribution measured in inclusive semileptonic decays of $B$ mesons to final states containing a charm quark, $B \rightarrow X_{c} \ell v$. The variable $n_{X}^{2}$ is a combination of the invariant mass of the charmed meson $m_{X}$, its energy in the $B$-meson rest-frame $E_{X, \mathrm{BRF}}$, and a constant $\tilde{\Lambda}=0.65 \mathrm{GeV}, n_{X}^{2}=m_{X}^{2} c^{4}-2 \tilde{\Lambda} E_{X, \mathrm{BRF}}+\tilde{\Lambda}^{2}$. The moments $\left\langle n_{X}^{k}\right\rangle$ with $k=2,4,6$ are measured as proposed by theory to constrain assumptions made in the theoretical description of inclusive observables in semileptonic $B$-meson decays. This description uses Heavy Quark Expansion (HQE), an effective QCD combined with an Operator Product Expansion.

The measurement is based on a sample of 231.6 million $e^{+} e^{-} \rightarrow \Upsilon(4 S) \rightarrow B \bar{B}$ events recorded with the BABAR experiment at the PEP-II $e^{+} e^{-}$-storage rings at SLAC. We reconstruct the semileptonic decay by identifying a charged lepton in events tagged by a fully reconstructed hadronic decay of the second $B$ meson. Correction procedures are derived from Monte Carlo simulations to ensure an unbiased measurement of the moments of the $n_{X}^{2}$ distribution. All moments are measured requiring minimum lepton momenta between $0.8 \mathrm{GeV} / c$ and $1.9 \mathrm{GeV} / c$ in the rest frame of the $B$ meson.

Performing a simultaneous fit to the measured moments $\left\langle n_{X}^{k}\right\rangle$ up to order $k=6$ combined with other measurements of moments of the lepton-energy spectrum in decays $B \rightarrow X_{c} \ell v$ and moments of the photon-energy spectrum in decays $B \rightarrow X_{s} \gamma$, we determine the quark-mixing parameter $\left|V_{c b}\right|$, the bottom and charm quark masses, the semileptonic branching fraction $\mathcal{B}\left(B \rightarrow X_{c} \ell v\right)$, and four non-perturbative heavy quark parameters. Using HQE calculations in the kinetic scheme up to order $1 / m_{b}^{3}$ we find

$$
\left|V_{c b}\right|=(41.65 \pm 0.43 \pm 0.40 \pm 0.58) \cdot 10^{-3} \text { and } m_{b}=(4.570 \pm 0.033 \pm 0.043) \mathrm{GeV} / c^{2},
$$

where the first uncertainty refers to experimental contributions, the second to uncertainties in the $\mathrm{HQE}$, and the third to theoretical uncertainties in the calculations of the semileptonic decay rate $\Gamma\left(B \rightarrow X_{c} \ell v\right)$. All obtained results are consistent with previous determinations. The inclusion of the moments $\left\langle n_{X}^{6}\right\rangle$ decreases the uncertainty on the HQE parameters $\mu_{\pi}^{2}$ and $\rho_{D}^{3}$. Furthermore, the theoretical treatment of higher order corrections in the HQE used for the moments $\left\langle m_{X}^{k}\right\rangle$ has been verified with these new measurements. 



\section{Kurzfassung}

Die vorliegende Arbeit präsentiert erste Messungen von Momenten der hadronischen $n_{X}^{2}$ Verteilung, gemessen in inklusiven semileptonischen $B$-Mesonenzerfällen in Endzustände mit einem charmQuark, $B \rightarrow X_{c} \ell v$. Die Variable $n_{X}^{2}$ ist eine Kombination der invarianten Masse des Mesons mit charm-Quark, $m_{X}$, seiner Energie im Ruhesystem des $B$-Mesons, $E_{X, \mathrm{BRF}}$, und einer Konstanten $\tilde{\Lambda}=0.65 \mathrm{GeV}, n_{X}^{2}=m_{X}^{2} c^{4}-2 \tilde{\Lambda} E_{X, \mathrm{BRF}}+\tilde{\Lambda}^{2}$. Diese Arbeit stellt die Messung der Momente $\left\langle n_{X}^{k}\right\rangle$ mit $k=2,4,6$ vor. Die theoretische Beschreibung von Observablen in semileptonischen $B$-Mesonenzerfällen erfordert Annahmen, welche durch diese Messung eingeschränkt werden können. Die Beschreibung benutzt die Heavy Quark Expansion (HQE), eine effektive QCD, die mit einer Operator Produkt Entwicklung kombiniert wird.

Die Messung basiert auf einem Datensatz von 231.6 Millionen $e^{+} e^{-} \rightarrow \Upsilon(4 S) \rightarrow B \bar{B}$ Ereignissen, die mit dem BABAR-Experiment an den PEP-II $e^{+} e^{-}$-Speicherringen am SLAC aufgezeichnet wurden. In Ereignissen, welche durch voll rekonstruierte hadronische Zerfälle des zweiten $B$ Mesons ausgewählt werden, wird der semileptonische Zerfall durch die Identifikation eines geladenen Leptons rekonstruiert. Aus Monte Carlo Simulationen werden Korrekturmethoden extrahiert, welche eine Messung der Momente der $n_{X}^{2}$ Verteilung ohne systematische Abweichung ermöglichen. Alle Momente werden mit minimalen Leptonimpulsen zwischen $0.8 \mathrm{GeV} / c$ und $1.9 \mathrm{GeV} / c \mathrm{im}$ Ruhesystem des $B$-Mesons gemessen.

Wir führen eine simultane Anpassung an die gemessenen Momente $\left\langle n_{X}^{k}\right\rangle$ bis zur Ordnung $k=6$ und an Messungen der Momente des Leptonenenergiespektrums in Zerfällen $B \rightarrow X_{c} \ell v$ und der Momente des Photonenenergiespektrums in Zerfällen $B \rightarrow X_{s} \gamma$ durch. Daraus bestimmen wir den Quarkmischungsparameter $\left|V_{c b}\right|$, die Massen der bottom- und charm-Quarks, das semileptonische Verzweigungsverhältnis $\mathcal{B}\left(B \rightarrow X_{c} \ell v\right)$ und vier nicht-perturbativen Parameter der HQE. Wir benutzen HQE Rechnungen im kinetischen Schema bis zur Ordnung $1 / m_{b}^{3}$ und finden

$$
\left|V_{c b}\right|=(41.65 \pm 0.43 \pm 0.40 \pm 0.58) \cdot 10^{-3} \text { und } m_{b}=(4.570 \pm 0.033 \pm 0.043) \mathrm{GeV} / c^{2},
$$

wobei der erste Fehler die experimentelle Unsicherheit angibt, der zweite die theoretische Unsicherheit und der dritte die zusätzliche theoretische Unsicherheit der Rechnungen der semileptonischen Zerfallsrate $\Gamma\left(B \rightarrow X_{c} \ell v\right)$. Alle Ergebnisse sind mit früheren Bestimmungen konsistent. Die Einbeziehung der Momente $\left\langle n_{X}^{6}\right\rangle$ verringert die Unsicherheit auf die HQE Parameter $\mu_{\pi}^{2}$ und $\rho_{D}^{3}$. Außerdem konnte die theoretische Behandlung von Korrekturen höherer Ordnung in der HQE für die Momente $\left\langle m_{X}^{k}\right\rangle$ verifiziert werden. 



\section{Contents}

\begin{tabular}{lll}
\hline Introduction & 1
\end{tabular}

2 Experimental Context of the Analysis 5

3 Theoretical Framework for Semileptonic B-Meson Decays and $\left|\mathbf{V}_{\mathbf{c b}}\right| \quad 9$

3.1 The Standard Model of Particle Physics . . . . . . . . . . . . . . . . 9

3.1.1 Mass Generation in the Standard Model . . . . . . . . . . . . 11

3.1.2 The Cabibbo-Kobayashi-Maskawa Matrix and the Unitarity Triangle 12

3.2 Heavy Quark Effective Theory . . . . . . . . . . . . . . . . . . 13

3.2 .1 General Remarks . . . . . . . . . . . . . . . . . . . . . . . 14

3.2 .2 From QCD to HQET . . . . . . . . . . . . . . . . 15

3.2.3 Description of Inclusive Semileptonic B-Meson Decays . . . . 17

3.2 .4 The Kinetic Scheme . . . . . . . . . . . . . . . . . . . . . . . . 19

3.3 Bottom Quark Mass Definitions . . . . . . . . . . . . . . . . 21

4 Outline of the Analysis Strategy 23

5 The BABAR Experiment 27

5.1 The PEP-II B-Meson Factory . . . . . . . . . . . . . . . . . . . . . . . . 27

5.2 The BABAR Detector . . . . . . . . . . . . . . . . . . . . . . . 28

5.2.1 Silicon Vertex Tracker . . . . . . . . . . . . . . . . 30

5.2 .2 Drift Chamber . . . . . . . . . . . . . . . 30

5.2 .3 Cherenkov Detector . . . . . . . . . . . . . . . 31

$5.2 .4 \quad$ Electromagnetic Calorimeter . . . . . . . . . . . . . . . . 31

5.2 .5 Instrumented Flux Return $\ldots \ldots \ldots$

6 Measurement of Moments of the Combined Mass-Energy Spectrum 35

6.1 Monte Carlo and BABAR Datasets . . . . . . . . . . . . . . . . 35

6.2 Reconstruction and Selection of the $B_{\text {reco }}$ Candidate . . . . . . . . . 36

$6.2 .1 \quad$ Kinematic Variables . . . . . . . . . . . . . . . . . . . 37

6.2 .2 Reconstruction Formalism . . . . . . . . . . . . . . . 38

6.2.3 Selection of one $B_{\text {reco }}$ Candidate per Event . . . . . . . . . . 39

6.3 Selection of Particles Originating from the Semileptonic Decay . . . . . . 39

6.3.1 Reconstruction of Charged Tracks . . . . . . . . . . . . . 40

6.3.2 Requirements for Neutral Particle Candidates . . . . . . . . . . . 41

6.3 .3 Particle Identification . . . . . . . . . . . . . . . . . . 43

6.4 Event Selection Criteria . . . . . . . . . . . . . . . . . . . . . . . . . . 45

6.5 Reconstruction of the Hadronic System . . . . . . . . . . . . . 47

6.5.1 Method of Least Squares with Constraints . . . . . . . . . . . 48

6.5.2 Linearization of Non-linear Problems . . . . . . . . . . . . 48 
6.5.3 Solution of the Linearized Problem . . . . . . . . . . . . . . . 49

6.5 .4 Use in This Analysis . . . . . . . . . . . . . . . . . . 50

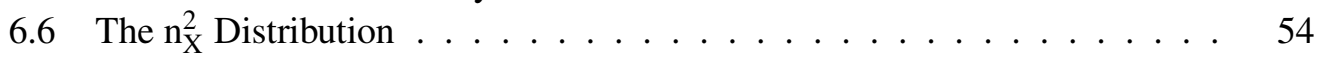

6.7 Background Subtraction $\ldots \ldots \ldots \ldots \ldots \ldots$

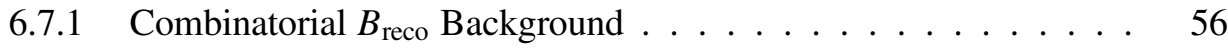

6.7 .2 Residual Background on the Signal Side . . . . . . . . . . . 57

6.7 .3 Verification of the Background Description . . . . . . . . . . 64

6.7 .4 Determination of Background Subtraction Weights . . . . . . 64

6.8 Extraction of Hadronic Moments . . . . . . . . . . . . . . . . . . . 67

$6.8 .1 \quad$ Situation Before Any Correction . . . . . . . . . . . . . 67

6.8 .2 Calibration Method . . . . . . . . . . . . . . . . . 70

6.8 .3 Remaining Bias Correction . . . . . . . . . . . . . . . . 74

6.8.4 Statistical Uncertainties and Correlations . . . . . . . . . . 74

6.8 .5 Calculation of Central Moments . . . . . . . . . . . 76

6.9 Investigations and Optimizations of the Moment Extraction . . . . . . 77

6.9.1 Comparison of Simulated with Data Distributions . . . . . . . . 77

6.9.2 Optimization of the Total Charge Requirement . . . . . . . . 81

6.9.3 Investigation of the Selected Region in $E_{\text {miss }}-c\left|\vec{p}_{\text {miss }}\right| \ldots \ldots$. . . 81

6.9 .4 Verification of the Calibration Procedure . . . . . . . . . 82

6.10 Results . . . . . . . . . . . . . . . . . . . . . . 85

$\begin{array}{llr}7 & \text { Evaluation of Systematic Uncertainties } & 89\end{array}$

7.1 Estimates for Systematic Uncertainties . . . . . . . . . . . . . . . . . 89

7.1.1 Statistical Uncertainty of the Combinatorial Background Subtraction 89

7.1.2 Statistical Uncertainty of the Simulated MC Sample . . . . . . . 89

7.1.3 Branching Fractions of Background Decays . . . . . . . . . . . . . 91

7.1 .4 Particle Identification . . . . . . . . . . . . . . . . . . . . . 92

7.1 .5 Track Selection Efficiency $\ldots \ldots \ldots$. . . . . . . . . . 93

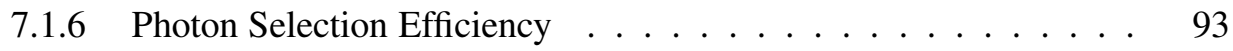

7.1 .7 Imperfection of the Moment Extraction Method . . . . . . . . . . 94

$7.1 .8 \quad$ Simulation Model of Signal Decays . . . . . . . . . . . . . . . 94

7.1 .9 Binning of Calibration Curves . . . . . . . . . . . . . . . 95

7.1 .10 Mismodeling of $E_{\text {miss }}-c\left|\vec{p}_{\text {miss }}\right| \ldots \ldots \ldots \ldots$. . . . . . . 97

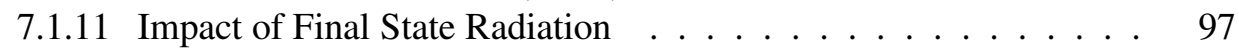

7.2 Summary of Systematic Uncertainties . . . . . . . . . . . . . . . . 98

7.3 Stability Studies . . . . . . . . . . . . . . . . . . . . . . . . 102

\begin{tabular}{llr}
\hline Interpretation of the Measurement & 105
\end{tabular}

8.1 The Fit Idea . . . . . . . . . . . . . . . . . . . . . . . . . . . . . . . . 105

8.2 Extraction Formalism . . . . . . . . . . . . . . . . . . 106

8.3 Theoretical Uncertainties . . . . . . . . . . . . . . . . . . . . 107

8.4 Experimental Correlations between Mass and Mass-Energy Moments . . 107

8.5 Results . . . . . . . . . . . . . . . . . . . . . . . . . . . . 108

8.5 .1 Experimental Input $\ldots \ldots \ldots \ldots \ldots$

$8.5 .2 \quad$ Fit Results . . . . . . . . . . . . . . . . . . . . . . . . 109 
8.5.3 Studies Using Different Input Moments . . . . . . . . . . . . 113

8.5 .4 Systematic Checks of the Fit Results . . . . . . . . . . . . . . 114

8.5.5 Comparison of Results with Previous Determinations . . . . . . . 117

9 Summary and Conclusion 121

\begin{tabular}{|ll} 
A Calibration Curves for $\mathrm{n}_{\mathrm{X}}^{4}$ and $\mathrm{n}_{\mathrm{X}}^{6}$ & 123
\end{tabular}

B Correlation Matrices for the Moments $n_{X}^{2}, n_{X}^{4}$, and $n_{X}^{6} \quad 127$

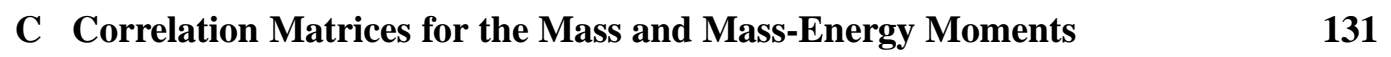

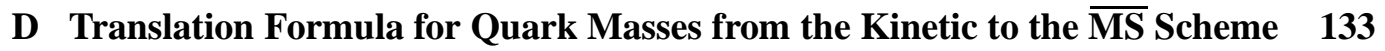

$\begin{array}{ll}\text { Bibliography } & 135\end{array}$ 



\section{List of Figures}

3.1 The Unitarity Triangle . . . . . . . . . . . . . . . . . . . . . . . . . 14

3.2 Feynman graphs of the decay $b \rightarrow c \ell v$ and $B \rightarrow X_{c} \ell v \ldots \ldots$

$4.1 \quad$ Illustration of the reconstructed event structure of the $\Upsilon(4 S)$ decay $\ldots .24$

5.1 Schematic view of the PEP-II storage rings . . . . . . . . . . . . 27

5.2 Integrated Luminosity for run1-6 . . . . . . . . . . . . . . . . . 28

5.3 Layout of the BABAR detector . . . . . . . . . . . . . . . . . . 29

5.4 SVT layout side view . . . . . . . . . . . . . . . . . . 30

5.5 Layout of the drift chamber . . . . . . . . . . . . . . . . 31

5.6 Schematic view of the DIRC $\ldots \ldots \ldots \ldots \ldots \ldots$

5.7 Longitudinal cross section of the EMC . . . . . . . . . . . 32

$6.1 \quad$ Number of $B_{\text {reco }}$ candidates per event . . . . . . . . . . . . . . . . . . . 39

6.2 Distributions of $m_{\mathrm{ES}}$ and $\Delta E$ for all $B_{\text {reco }}$ modes . . . . . . . . . . 39

6.3 Dependence of $m_{\text {miss }}^{2}$ and $E_{\text {miss }}-c\left|\vec{p}_{\text {miss }}\right|$ resolution on $\vec{p}_{v \text {,true }} \ldots \ldots . . .46$

6.4 Energy resolution of the $X_{c}$ system used for the kinematic fit. . . . . . . . 52

6.5 Resolution of all four components of the $X_{c}$ four-vector . . . . . . . . 53

6.6 Probability of $\chi^{2}$ for the final fit performed on data . . . . . . . . . 53

6.7 Resolution before and after the kinematic fit of $n_{X}^{2}, E_{X, \mathrm{BRF}}$, and $m_{X} \ldots . \quad 54$

$6.8 n_{X}^{2}$ distribution on generator level . . . . . . . . . . . . . 55

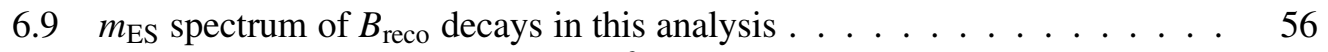

$6.10 m_{\mathrm{ES}}$ sideband scaling factors and $n_{X}^{2}$ distributions in the sideband . . . . 58

6.11 Breakdown of the residual background on the signal side . . . . . . 60

6.12 Breakdown of the residual background for subsets of the MC dataset . . . 61

6.13 Feynman graphs for the production of right-flavored of $D_{s}$ or $D$ mesons . 61

6.14 Plots for the background verification for charged $B$ mesons. . . . . . . . . 65

6.15 Plots for the background verification for neutral $B$ mesons. . . . . . . . 66

6.16 Measured $n_{X}^{2}$ spectra in data and background subtraction weights . . . . 68

6.17 MC Comparison of true and measured moments after the kinematic fit . . 69

6.18 MC Comparison of true and measured moments; electrons and muons . . 69

6.19 Comparison of the momentum misreconstruction for $e$ and $\mu$. . . . . 70

6.20 MC Comparison of true and measured moments; $\left|\vec{p}_{\ell}\right|$ restriction . . . . 70

6.21 Calibration curves for $n_{X}^{2}$; electrons . . . . . . . . . . . . . 72

6.22 Calibration curves for $n_{X}^{2}$; muons . . . . . . . . . . . . 73

6.23 Bias after application of the calibration on simulated signal decays . . . . 74

6.24 Data MC comparison for $E_{\text {miss }}-c\left|\vec{p}_{\text {miss }}\right| \ldots \ldots \ldots$. . . . . . . 78

6.25 Data MC comparison for the charged multiplicity of the $X_{c}$ system . . . 79

6.26 Data MC comparison for the neutral multiplicity of the $X_{c}$ system . . . 79

6.27 Data MC comparison for the neutral multiplicity binned in $E_{\text {neutral }} \ldots$. . . 80 
6.28 Data MC comparison for $n_{X}^{2} \ldots \ldots \ldots \ldots$. . . . . . . . . . . .

$6.29 Q_{\text {tot,event }}$ in the selected event sample in data $\ldots \ldots \ldots . \ldots . \ldots 82$

6.30 Mean and RMS of the distribution $n_{X, \text { true }}^{2}-n_{X, \text { reco }}^{2}$ in bins of $E_{\text {miss }}-c\left|\vec{p}_{\text {miss }}\right| \quad 83$

6.31 Verification of the analysis procedure on splitted MC samples . . . . . . 84

6.32 Calibration verification procedure with exclusive modes . . . . . . . . . . 84

6.33 Measured moments $\left\langle n_{X}^{2}\right\rangle,\left\langle n_{X}^{4}\right\rangle,\left\langle n_{X}^{6}\right\rangle$, and the central moments. . . . . . 86

6.34 Color map of correlation coefficients . . . . . . . . . . . . . 87

7.1 Moments Distribution performing calibration/bg. subtraction Toy studies . 90

7.2 Background component due to fake muons . . . . . . . . . . . . . 92

7.3 Variation of moments with track and neutral killing . . . . . . . . 93

7.4 Systematic variation due to signal branching fractions . . . . . . . . . . 96

7.5 Impact of $E_{\text {miss }}-c\left|\vec{p}_{\text {miss }}\right|$ cut variation . . . . . . . . . . . . . . . 97

7.6 Comparison of moments when excluding FSR events from the MC samples 98

7.7 Moments of independent subsets on data . . . . . . . . . . . 103

7.8 Normalized comparison of binned moments of independent subsets on data 104

8.1 Comparison of fit result and measurement for hadronic moments . . . . . 110

8.2 Comparison of fit result and measurement for lepton-energy moments . . 111

8.3 Comparison of fit result and measurement for photon-energy moments . . 111

8.4 Comparison of results for $\left|V_{c b}\right|$ and $m_{b}$ for the different performed fits . . 114

8.5 Comparison of results for $\mu_{\pi}^{2}$ and $\rho_{D}^{3}$ for the different performed fits . . 115

8.6 Comparison of two different fits in the $\mu_{\pi}^{2}-m_{b},\left|V_{c b}\right|-m_{b}$, and $\mu_{\pi}^{2}-\rho_{D}^{3}$ plane . 115

8.7 Pull and $\chi^{2}$ distributions for Toy MC studies of the HQE fit . . . . . . . . 116

8.8 Impact of theory uncertainties of fit results $\ldots \ldots \ldots \ldots$

8.9 Comparison of four different fits in the $\mu_{\pi}^{2}-m_{b}$ plane . . . . . . . . . . 119

A.1 Calibration curves for $n_{X}^{4}$; electrons . . . . . . . . . . . . 123

A.2 Calibration curves for $n_{X}^{4} ;$ muons . . . . . . . . . . . . . . . . 124

A.3 Calibration curves for $n_{X}^{6}$; electrons . . . . . . . . . . . . 125

A.4 Calibration curves for $n_{X}^{6}$; muons . . . . . . . . . . . . 126 


\section{List of Tables}

6.1 Summary of $b \rightarrow c \ell v$ branching fractions in MC $\ldots \ldots 36$

6.2 Summary of datasets used in the analysis . . . . . . . . . . . . . 37

6.3 Summary of rejection rates for the charged track selection criteria . . . . 42

6.4 Summary of rejection rates for the neutral candidate selection criteria . . 43

6.5 Number of events remaining after each event selection cut . . . . . . . . 47

6.6 Resolution before and after the kinematic fit of $n_{X}^{2}, E_{X, \mathrm{BRF}}$, and $m_{X} \ldots . .54$

6.7 Summary of scaling factors used to correct background processes in MC . $\quad 59$

6.8 Number of signal and background events for all cuts on $p_{\ell, \mathrm{BRF}} \ldots \ldots 5$

7.1 Variation factors of the relative branching fractions for signal decays . . . 95

7.2 Central values and uncertainties for the $\left\langle n_{X}^{2}\right\rangle$ for all cuts $p_{\ell \text { BRF }} \ldots \ldots$. . . 99

7.3 Central values and uncertainties for the $\left\langle n_{X}^{4}\right\rangle$ for all cuts $p_{\ell \text { BRF }} \ldots \ldots$

7.4 Central values and uncertainties for the $\left\langle n_{X}^{6}\right\rangle$ for all cuts $p_{\ell, \mathrm{BRF}} \ldots \ldots . .101$

8.1 HQE fit results with experimental and theoretical uncertainties. . . . . . . 112

B.1 Correlation matrix of the moments $\left\langle n_{X}^{2}\right\rangle$ and $\left\langle n_{X}^{4}\right\rangle \ldots \ldots \ldots \ldots$

B.2 Correlation matrix of the moments $\left\langle n_{X}^{2}\right\rangle,\left\langle n_{X}^{4}\right\rangle$, and $\left\langle n_{X}^{6}\right\rangle \ldots \ldots . \ldots 129$

C.1 Correlation matrix of the moments $\left\langle n_{X}^{2}\right\rangle,\left\langle n_{X}^{4}\right\rangle,\left\langle m_{X}^{2}\right\rangle$, and $\left\langle m_{X}^{4}\right\rangle \ldots 132$ 



\section{Introduction}

At the beginning of the 21st century, the Standard Model of particle physics represents our understanding of the fundamental nature of the universe. It describes the basic constituents all matter is made of, which are three families of quarks and leptons, and two of three forces, the strong force and the electroweak force, that act upon them. Gravity, the force acting on large scales, is negligible for the description of the interactions of elementary particles and is not incorporated in the Standard Model but described by general relativity. This fundamental approach has been developed in a strong interplay between experimental discoveries and precision measurements on the one hand and theoretical ideas on the other hand. The wish for a simple and consistent description of all observed phenomena has lead to a quantum field theory that successfully describes many physical observables. However, there are several shortcomings of the Standard Model that indicate that the current description is not "the end of the story". Still, there are questions unsolved nowadays, that are, for example, the origin of the matter-antimatter asymmetry in the universe, the origin of mass of elementary particles, and the somehow arbitrary number of three families of the existing particles. New experiments, such as those that will operate at the Large Hadron Collider at CERN from 2008 on, are built to answer some of these questions.

The mechanism of quark mixing is a fundamental pillar of the Standard Model. This formalism successfully describes transitions between the quark families. It makes use of the Cabibbo-Kobayashi-Maskawa Matrix, a $3 \times 3$ unitary matrix, which can be parametrized by four independent parameters. These parameters are four of the 18 free parameters of the Standard Model and have to be measured in experiments. A precise determination of the Standard Model parameters allows to test predictions derived from these input numbers. Furthermore, future theories beyond the Standard Model might have a reduced number of free parameters, thus, calculate one of the Standard Model parameters from more fundamental considerations. They can be tested by the precise determination of the parameters beforehand.

The BABAR experiment operating at the PEP-II $e^{+} e^{-}$-storage rings at the Stanford Linear Accelerator Center (SLAC) in California and its competitor, the Belle experiment at KEK in Japan, have played a leading role in the precise determination of Standard Model parameters, in particular those describing $C P$ violation in the Standard Model, which was found to be able to explain the observed $C P$ violation in decays of neutral $B$ mesons. Furthermore, the measurements of quark mixing parameters including bottom quarks, such as $\left|V_{c b}\right|$ and $\left|V_{u b}\right|$, describing the transition of a bottom quark into a charm or an up quark, respectively, have been driven significantly by both collaborations.

This thesis presents a determination of the quark mixing parameter $\left|V_{c b}\right|$. Moreover, the masses of the involved charm and bottom quarks, also free parameters of the Standard Model, are determined in this work. Properties of bottom quarks can be best measured in decays of $B$ mesons, bound states containing one bottom antiquark and a light up or down quark. The most successful method to determine these parameters in the last years uses inclusive semileptonic $B$-meson decays to hadronic final states containing a charm 
quark, $B \rightarrow X_{c} \ell v$. The measured observables, these are the total decay rate and moments of the lepton-energy and hadronic-mass distribution, can be described reliably by an effective theory of quantum chromodynamics (QCD) using an Operator Product Expansion. The theoretical predictions of this so-called Heavy Quark Expansion (HQE) are fitted to the measured moments and yield results for the quark masses and four additional HQE parameters. These are used to extract $\left|V_{c b}\right|$ from a calculation of the total semileptonic decay rate.

Moments of the hadronic-mass distribution have been measured by several collaborations so far [1-5]. However, theorists have proposed the measurement of another hadronic observable [6]. This variable $n_{X}^{2}$ is a combination of the hadronic mass $m_{X}$, its energy in the $B$-meson rest-frame $E_{X, \mathrm{BRF}}$, and a constant $\tilde{\Lambda}=0.65 \mathrm{GeV}$,

$$
n_{X}^{2}=m_{X}^{2} c^{4}-2 \tilde{\Lambda} E_{X, \mathrm{BRF}}+\tilde{\Lambda}^{2} .
$$

Calculations for moments of this $n_{X}^{2}$ distribution are expected to be better controlled theoretically and thereby yield a higher sensitivity to two of the four HQE parameters. As these are used in the determination of $\left|V_{c b}\right|$, its precision might also increase with exploiting the additional experimental information. Moreover, testing the HQE in these moments of the $n_{X}^{2}$ distribution allows to verify the treatment of higher order corrections appearing in the expansion of the hadronic-mass moments. This is due to the fact that the theoretical expansion of the $n_{X}^{2}$ moments converges better even without those corrections which are needed for the mass moments [7]. In summary, the Heavy Quark Expansion can be further tested by the measurement of the moments of the $n_{X}^{2}$ distribution and additional information on this theoretical framework can be gained. As moments of the $n_{X}^{2}$ distribution have not been measured so far, this thesis presents a first measurement of the moments $\left\langle n_{X}^{k}\right\rangle$ with $k=2,4,6$.

The measurement uses $B$ mesons produced in the reaction $e^{+} e^{-} \rightarrow \Upsilon(4 S) \rightarrow B \bar{B}$ at the PEP-II $e^{+} e^{-}$-storage rings at SLAC. Their decay products are detected with the BABAR detector. As this measurement aims at an inclusive measurement of all possible decays $B \rightarrow X_{c} \ell v$, we do not reconstruct the hadronic final state explicitly. Therefore, other criteria to associate measured particles to the hadronic system are needed. For that reason, the second $B$ meson in the event is reconstructed first in purely hadronic decay modes. Then, a charged lepton from the semileptonic $B$-meson decay is searched within the remaining particles. The rest of the measured particles are combined to form the hadronic system. Although from a theoretical point of view, a measurement as inclusive as possible is desirable, the reconstruction and identification of low energetic leptons is difficult. Furthermore, more leptons originating from background decays contribute at low lepton momenta. Therefore, the moments $\left\langle n_{X}^{k}\right\rangle(k=2,4,6)$ are measured as a function of the minimal lepton momentum, starting at $p_{\ell, \mathrm{BRF}} \geq 0.8 \mathrm{GeV} / c$ up to momenta of $p_{\ell, \mathrm{BRF}} \geq 1.9 \mathrm{GeV} / c$. This dependence of the moments on the minimal lepton momentum is also predicted by theory.

The measured moments $\left\langle n_{X}^{k}\right\rangle$ are added to the fit of theory predictions to the abovementioned lepton-energy and hadronic-mass moments. Their impact on the extracted parameters is studied and the fit procedure is verified. The extracted parameters are compared to other determinations.

This thesis is organized as follows. 
Chapter 2 gives a brief overview of the history and current status of measurements of the quark mixing parameter $\left|V_{c b}\right|$. Both, theoretical and experimental developments are discussed. The chapter ends with the recent state of the art of the determination of $\left|V_{c b}\right|$, yielding the basis for the presented analysis.

Chapter 3 introduces the theoretical concepts needed for the description of $B$-meson decays. Therefore, a short introduction into the Standard Model of particle physics is given, implementing the concept of quark mixing. Finally, an effective theory is developed from quantum chromodynamics, yielding the framework to derive observables in $B$-meson decays. Parameters of this effective theory are derived in the framework of an Operator Product Expansion. Furthermore, bottom quark masses, defined in different renormalization schemes of the QCD, are discussed.

Chapter 4 reports about the design and performance of the BABAR experiment, which is used to detect the decay products of the $B$ mesons investigated in this thesis. The PEP-II $e^{+} e^{-}$-storage rings and all subdetectors are described briefly.

Chapter 5 gives a short outline of the analysis method used for the measurement of the hadronic moments of the $n_{X}^{2}$ distribution. It relates the presented measurement to previous, similar measurements and summarizes the general steps performed in the analysis.

Chapter 6 describes the measurement in more detail. Datasets used in this measurement and basic reconstruction criteria are introduced. Reconstruction principles of the two $B$ mesons in each event and event selection criteria are discussed. In the following, the method to subtract background contributions both from the data itself and from MC simulations is discussed. Studies performed on MC simulations are described, which give insight into the main reasons for the misreconstruction and distortion of the hadronic system and yield tools to correct them. These tools are in turn verified on independent MC datasets to give confidence in the robustness of the moment extraction procedure. Afterwards, the moment measurement on data is described.

Chapter 7 summarizes the evaluation of systematic uncertainties that have been considered in the moments measurement. The obtained uncertainties are discussed. Crosschecks by dividing the data sample in independent subsets are described.

Chapter 8 describes how the measured moments are interpreted in the framework of a Heavy Quark Expansion. A fit of these theory predictions to our measurement is performed to extract Standard Model parameters and HQE parameters. Several test verifying the validity of the fit are performed. The results for the fit parameters are compared to previous determinations.

Chapter 9 summarizes the results and presents the conclusions. 



\section{Experimental Context of the Analysis}

This chapter will give a brief overview of the development of the determination of the quark mixing parameter $\left|V_{c b}\right|$. Both, theoretical and experimental developments are discussed, which of course have been closely related. The chapter will end with the recent state of the art of the determination of $\left|V_{c b}\right|$, yielding the basis for the presented analysis.

When Kobayashi and Maskawa in 1973 extended Cabibbo's idea of quark mixing to the third family, the size of the matrix element $\left|V_{c b}\right|$ was still unknown. The $b$ quark was not discovered until 1977 [8], and for an extraction of $\left|V_{c b}\right|$ its lifetime and the semileptonic branching fraction of $b$ quarks to charm final states were needed. A simple quark-level formula for $W$ emission, using the so-called spectator model, was used to connect the lifetime to $\left|V_{c b}\right|$ via the total semileptonic decay rate $\Gamma_{b \rightarrow x \ell v}$,

$$
\Gamma_{b \rightarrow x \ell v}=\frac{G_{F} m_{b}^{5}}{192 \pi^{3}} \eta_{\mathrm{QCD}}\left(F\left(\frac{m_{c}}{m_{b}}\right)\left|V_{c b}\right|^{2}+F\left(\frac{m_{u}}{m_{b}}\right)\left|V_{u b}\right|^{2}\right)
$$

where the coefficients $F$ are phase space factors based on constituent quark masses and $\eta_{\mathrm{QCD}}$ are $\mathrm{QCD}$ corrections.

While the first decays of $B$ mesons to leptons were observed in 1981 by the CLEO and CUSB collaborations [9-11] with uncertainties in the 30 percent region, the $b$-quark lifetime was measured in 1983 by the MAC and Mark II collaborations [12,13]. Together, these measurements yielded the first measurement of $\left|V_{c b}\right|$, much more precise than the loose bounds given before. Using these measurements ref. [14] determines $\left|V_{c b}\right|$ in the range from $49.7 \cdot 10^{-3}$ to $76.0 \cdot 10^{-3}$ for lifetimes between 1.4 and $0.6 \mathrm{psec}$. The size of $\left|V_{u b}\right|$ could be neglected as it was already known that $\Gamma_{b \rightarrow u} / \Gamma_{b \rightarrow c} \leq 0.05$ (cf. references in [14]). The phase space factors $F$ were associated with uncertainties in the $10 \%$ region. The term $m_{b}^{5}$ yielded with the rather unknown $b$-quark mass an uncertainty of $30 \%$. As also the experimental precision on the $b$ decay rate at that time was still low, a high precision of the QCD corrections $\eta_{\mathrm{QCD}}$ was not considered to be relevant.

When first exclusive measurements of semileptonic decays of $B$ mesons, specifically the decay $B \rightarrow D^{*} \ell v$, and their polarization became feasible by the ARGUS collaboration in 1987-89 [15, 16], another access to $\left|V_{c b}\right|$ became available. The first method to extract $\left|V_{c b}\right|$ from exclusive decays was to use the branching fraction together with polarization measurements. It yielded an uncertainty on $\left|V_{c b}\right|$ in the $20 \%$ region [17].

For the calculation of exclusive decay rates, form-factors are needed which were calculated in different models [18-20]. In 1989/90, the advent of the Heavy Quark Effective Theory (HQET) promised a model-independent way to extract $\left|V_{c b}\right|$ from exclusive $B$ meson decays [21,22]. The decay $B \rightarrow D^{*} \ell v$ could be described with only one universal form-factor, the Isgur-Wise function $\mathcal{F}(w)$, where $w$ is the boost of the $D^{*}$ in the $B$-meson rest-frame. This new approach used the exact prediction of this form-factor at $w=1$, the socalled zero-recoil of the $D^{*}$ in the $B$-meson rest-frame. As, unfortunately, the phase space is empty at this point, the spectrum $\mathrm{d} \mathcal{B} / \mathrm{d} w$ is measured and extrapolated to $w=1$. The first 
determination of the product $\left|V_{c b}\right| \cdot \mathcal{F}(1)$ in combination with the parameter $\rho^{2}$, entering in the parametrization of the spectrum $\mathrm{d} \mathcal{B} / \mathrm{d} w$, was performed in 1993 [23]. However, this method lead to uncertainties which were not competitive to the previous determinations due to large statistical uncertainties.

Since that time, the extraction of $\left|V_{c b}\right|$ from exclusive decays $B \rightarrow D^{*} \ell v$ has undergone a great development as it has been measured by many collaborations. Averaging all measurements yields in summer 2007 the value $\left|V_{c b}\right| \cdot \mathcal{F}(1)=(35.89 \pm 0.56) \cdot 10^{-3}$ [24], the uncertainty has reached the $2 \%$ level. This improvement has been possible due to an improved parametrization of the spectrum $\mathrm{d} \mathcal{B} / \mathrm{d} w$ [25], which requires measured form-factor ratios $R_{1}(1)$ and $R_{2}(1)$ as input. These measurements still yield a large uncertainty on the parameters describing this decay.

In parallel to this exclusive approach, in the inclusive part of the $\left|V_{c b}\right|$ measurements it became clear that with increasing precision of the $\tau_{b}$ measurements the predictions for the $b$-quark mass and the QCD corrections to the calculations of the semileptonic decay rate will become more and more important. Another effective QCD, the Heavy Quark Expansion (HQE), was the tool to provide calculations which remarkably decreased the uncertainties on these parameters, as it showed how to extract them from other observables. The first papers on HQE predictions of the inclusive semileptonic decay rate were published in 1992/93 [26, 27]. They formulated the idea to parametrize the QCD corrections to the semileptonic decay rate with fundamental HQE parameters, which then on turn can be extracted from moment measurements of hadronic and leptonic spectra. Also the $b$-quark mass enters in the expansion of these moments and thus can be extracted. For this purpose, the measurement of moments of the lepton-energy spectrum and of the hadronicmass spectrum in decays $B \rightarrow X_{c} \ell v$ became relevant. This idea turned out to be a very successful way to extract $\left|V_{c b}\right|$ from semileptonic $B$-meson decays.

The calculations for the semileptonic decay rate and the moments of the lepton-energy and hadronic-mass spectrum were carried out in two different renormalization schemes, the 1S- and the kinetic scheme (compare to section 3.2.3). Explicit calculations were first published in the 1S-scheme in 1997 [28]. They were used with further improvements [29] by the CLEO collaboration in 2001/2002 [30,31] to fit the measured hadronic-mass and lepton-energy moments and extract $\left|V_{c b}\right|$ with a total uncertainty of about $3 \%$. The first concrete calculations for the semileptonic decay rate in the kinetic scheme of the HQE became available in 2003 [32]. They were followed by calculations for the moments of the lepton-energy and hadronic-mass spectrum in 2004 which were still improved over the years. More moments measurements became available at the same time by several collaborations and are still worked on today $([4,33,34]$ and $[1-5])$. Thus, theoretical improvements are also still underway, as the experimental data becomes more and more accurate. The evolution of the $\left|V_{c b}\right|$ extraction from inclusive decays started with fits of theory calculations to moment measurements of one single experiment $[31,35]$, while now, as more measurements are published, global fits to the whole set of measurements are done, decreasing the experimental uncertainty significantly $[24,36]$. The uncertainty is at the moment in the order of 1.5 to $2 \%$ dominated by the theoretical uncertainty on the expansion of the semileptonic decay rate.

So far, no discrepancy of the theory predictions for the moments and the measurement became evident. The HQE parameters $\mu_{G}^{2}$ and $\rho_{\mathrm{LS}}^{3}$ extracted from the moments fits can 
be compared to other determinations and they are found to be consistent. $\left|V_{c b}\right|$ extracted from inclusive decays is in agreement with the value extracted from exclusive decays on the $1.6 \sigma$ level. It is at the moment not clear where this discrepancy comes from, and, as it is not very significant, further investigation is needed here. Important for parametrizations of shape functions needed in the calculations of partial decay rates in decays $b \rightarrow u \ell v$ are the parameters $m_{b}$ and $\mu_{\pi}^{2}$, yielding a reason to improve the current knowledge even further. The measurement of further hadronic moments, such as those of the distribution of the variable $n_{X}^{2}$, as proposed in [6] and measured in this analysis, are supposed to help constraining the HQE parameters and therefore may lead to an improved measurement of $\left|V_{c b}\right|$ and $m_{b}$. Furthermore, the expansion of hadronic moments in the framework of the HQE, especially the treatment of higher order corrections, can be tested in a suitable observable. 



\section{Theoretical Framework for Semileptonic B-Meson Decays and $\left|\mathbf{V}_{\mathbf{c b}}\right|$}

This chapter gives a brief introduction into the main theoretical concepts describing $B$ meson decays and their observables reconstructed in this analysis. To define the framework, a short introduction into the Standard Model of Particle Physics is given, focusing on concepts typically used in $B$-meson physics. That is in particular the concept of quark mixing derived from the fundamental Higgs mechanism needed for the mass generation of elementary particles. This general discussion is followed by an introduction to the Heavy Quark Effective Theory suited to describe decays of $B$ mesons. A short review of $b$-quark mass definitions is given at the end.

\subsection{The Standard Model of Particle Physics}

The Standard Model of Particle Physics (SM) is a theory of fundamental particles and the electromagnetic, weak, and strong interactions that act upon them. Together with the theory of general relativity that describes gravity, the SM provides a description of nature that so far accounts for almost all observed phenomena in particle physics. However, the SM fails in describing a few observations, such as non-zero neutrino masses, and it needs the large number of 18 free parameters which cannot be derived from first principles. Furthermore, there is no convenient way to combine the SM with the theory of general relativity. The interpretation of this shortcoming is that both are large and small scale limits of a more fundamental theory, respectively. Many attempts to formulate such an underlying theory, e. g. supersymmetry extensions of the SM or string theory, have been made, and need to be driven by new experimental indications of physics beyond the SM. The experimental detection or exclusion of such new phenomena and their theoretical description are the aim of many of today's experiments.

The interactions of the Standard Model are described by the $S U(3)_{C} \times S U(2) \times U(1)_{Y}$ group, where the $S U(3)_{C}$ subgroup characterizes the strong interaction coupling to the color quantum numbers and the $S U(2) \times U(1)_{Y}$ subgroup describes the mixed electromagnetic and weak interactions as formulated in the Glashow-Weinberg-Salam (GWS) model (cf. [37-39] reviewed in [40,41]). The $S U(2)$ part of the weak interaction gives rise of a triplet of vector bosons $\mathbf{W}^{\mu}$ associated with the quantum number of weak isospin. To the $U(1)$ component contributes one single boson $B^{\mu}$ associated with the weak hypercharge $Y$, a combination of the electric charge $Q$ and the third component of the weak isospin $I_{3}$, $Y=2\left(Q-I_{3}\right)$.

With the current experimental knowledge, matter is built of two types of fermions, quarks and leptons, both having spin $1 / 2$ and interacting via the exchange of gauge bosons with integer spin. The six leptons can be arranged in three generations with increasing 
mass:

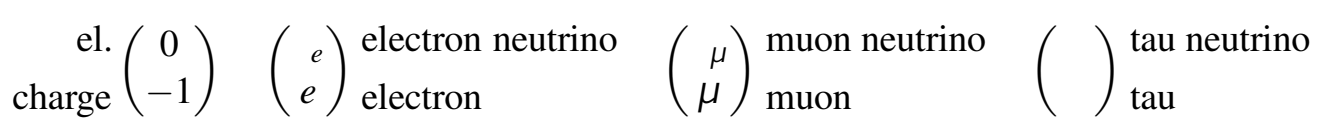

Similarly, there are six quantum numbers for quarks, called flavors, and they can as well be arranged in three mass-generations:

$$
\begin{array}{r}
\text { el. } \\
\text { charge }\left(\begin{array}{l}
+2 / 3 \\
-1 / 3
\end{array}\right) \quad\left(\begin{array}{l}
u \\
d
\end{array}\right) \text { up quark } \\
\text { down quark }
\end{array}\left(\begin{array}{l}
c \\
s
\end{array}\right) \begin{aligned}
& \text { charm quark } \\
& \text { strange quark }
\end{aligned}\left(\begin{array}{l}
t \\
b
\end{array}\right) \begin{aligned}
& \text { top quark } \\
& \text { bottom quark }
\end{aligned}
$$

However, this illustration of the elementary particles only accounts for their mass and charge properties, which are relevant for a descriptive definition of particles. Furthermore, whenever weak processes such as muon, neutron, or pion decay were investigated in the beginning of particle physics measurements, only transitions within one generation were observed, making this picture very successful. Consequently, the weak interaction has been formulated such that it only mediates transitions within one generation. While for the leptons, this argument remained valid up to the current knowledge, in the progress of measurements in the quark sector, a different behavior was observed. There, also transitions between the above-mentioned generations were observed, e.g. the decay of the lightest kaon. To assure the lepton-quark universality of the weak interaction, that is the equivalent description of the weak interaction for leptons and quarks, the quark states have been rearranged to doublets of so-called weak eigenstates,

$$
\left(\begin{array}{l}
u \\
d
\end{array}\right)\left(\begin{array}{l}
c \\
s
\end{array}\right)\left(\begin{array}{l}
t \\
b
\end{array}\right) \longrightarrow\left(\begin{array}{c}
u \\
d^{\prime}
\end{array}\right)\left(\begin{array}{c}
c \\
s^{\prime}
\end{array}\right)\left(\begin{array}{c}
t \\
b^{\prime}
\end{array}\right) \text {. }
$$

In this formulation, the coupling of the weak interaction within one generation remains valid, and, thereby, allows an elegant description of the electroweak interaction.

The transformation between the weak and the mass eigenstates of the quarks is formulated by the Cabibbo-Kobayashi-Maskawa (CKM) Matrix, a concept discussed in subsections 3.1.1 and 3.1.2.

Similarly, the vector bosons $\mathbf{W}^{\mu}$ and $B^{\mu}$, introduced above as the fundamental gauge fields, are not the physical fields observable in interactions. These can be obtained by rotations,

$$
\begin{aligned}
& W^{ \pm}=\frac{W^{1} \mp i W^{2}}{\sqrt{2}}, \\
& Z^{0}=\cos \left(\theta_{W}\right) W^{3}-\sin \left(\theta_{W}\right) B, \\
& A=\sin \left(\theta_{W}\right) W^{3}+\sin \left(\theta_{W}\right) B,
\end{aligned}
$$

introducing the Weinberg mixing angle $\theta_{W}$ with $\sin \theta_{W} \approx 0.231$ [42]. These observable fields mediate different interactions regarding the electric charge; the $W^{ \pm}$bosons mediate the so-called charged current interaction, while the $Z^{0}$ and photon field $A$ are responsible for neutral current interactions. A striking observation regarding these gauge bosons is, however, the large mass differences among them. While the photon is massless, the $W^{ \pm}$ bosons have a mass of about $80 \mathrm{GeV} / c^{2}$ and the $Z^{0}$ of $91 \mathrm{GeV} / c^{2}$ [42]. Furthermore, the $\mathrm{SM}$ as introduced so far even forbids massive gauge bosons due to gauge invariance, a fact which is obviously not observed in the experiment. 


\subsubsection{Mass Generation in the Standard Model}

The striking inconsistency of the masses of the gauge bosons seeks for a satisfying mechanism to explain these properties observed in experiments. In the SM, this is provided by the so-called Higgs mechanism [43-45]. In this model, the mass is generated by the interaction of particles with the Higgs field, represented by an isospin doublet $(I=1 / 2, Y=1)$ of a scalar Higgs particle $\Phi=\left(\phi^{+}, \phi^{0}\right)$, with $\phi^{+}$and $\phi^{0}$ complex fields, fulfilling the Lagrangian density

$$
\mathcal{L}_{\text {Higgs }}=\left(D^{\alpha} \Phi\right)^{\dagger}\left(D_{\alpha} \Phi\right)-V\left(\Phi^{\dagger}, \Phi\right) \quad \text { with } \quad V\left(\Phi^{\dagger}, \Phi\right)=-\mu^{2} \Phi^{\dagger} \Phi+\lambda^{2}\left(\Phi^{\dagger} \Phi\right)^{2} .
$$

The potential $V$ has rotational symmetry and has its minimum on a circle at

$$
|\Phi|=\frac{v}{\sqrt{2}} \quad \text { with } \quad v=\mu / \lambda .
$$

This means that, in principle, any state with $|\Phi|^{2}=v^{2} / 2$ could be the ground-state in this potential. However, for an interpretation of physical states a specific gauge is convenient, namely that one revealing a massless photon, massive $W$ and $Z$ bosons and a Higgs particle.

For our discussion, we choose a ground state $\Phi=(0, v / \sqrt{2})$ breaking the symmetry of the underlying Lagrangian. Regarding a small excitation of this ground state,

$$
\Phi(x)=\frac{1}{\sqrt{2}}\left(\begin{array}{c}
0 \\
v+\eta(x)
\end{array}\right)
$$

and plugging in the covariant derivative for this case, yields the Lagrangian density,

$$
\begin{aligned}
\mathcal{L}= & {\left[\frac{1}{2}\left(\partial^{\mu} \eta\right)\left(\partial_{\mu} \eta\right)-\mu^{2} \eta^{2}\right]-\frac{1}{4} F_{\mu \nu}^{i} F^{i \mu v}-\frac{1}{4} f_{\mu \nu} f^{\mu v} } \\
& +\frac{1}{2} \cdot \frac{g^{2} v^{2}}{4}\left(\left|W_{\mu}^{(+)}\right|^{2}+\left|W_{\mu}^{(-)}\right|^{2}\right)+\frac{1}{2} \cdot \frac{v^{2}}{4}\left|g^{\prime} B_{\mu}-g W_{3 \mu}\right|^{2} .
\end{aligned}
$$

Here $F_{\mu \nu}^{i}$ and $f_{\mu \nu}$ are the field strength tensors of the $\mathbf{W}_{\mu}$ and $B_{\mu}$ gauge boson fields. This form displays those interesting facts, which this theoretical construction aims at:

1. A neutral Higgs particle exists, having the mass $m_{\text {Higgs }}=\sqrt{2} \mu$.

2. The $W$ bosons have the mass $M_{W}=g v / 2$.

3. The mass of the $Z^{0}$ boson is connected to the mass of the $W$ via $M_{Z}=M_{W} / \cos \theta_{W}$.

4. There is no mass term for the electromagnetic potential $A_{\mu}$. The photon remains massless.

The charged fermions get their mass through their coupling to the Higgs field, symbolized by a term in the Lagrangian $\mathcal{L}(f, \Phi)$ which writes explicitly as [46,47],

$$
\begin{aligned}
\mathcal{L}(f, \Phi)= & \sum_{j=1}^{3}\left(m_{\ell}\right)_{j} \bar{\ell}_{L}^{j} \Phi \ell_{R}^{j} \\
& +\sum_{j, k=1}^{3}\left[\left(m_{U}\right)_{j k} \bar{u}_{L}^{j} \Phi u_{R}^{k}+\left(m_{D}\right)_{j k} \bar{d}_{L}^{j} \Phi^{c} d_{R}^{k}\right]+\text { h. c.. }
\end{aligned}
$$


Here, the sum runs over the generations, and the parameters $\left(m_{\ell}\right)_{j}$ and $\left(m_{U / D}\right)_{j k}$ are the Yukawa couplings of the Higgs field to the leptons and up- or down-type quarks, respectively. While the lepton mass matrix $\left(m_{\ell}\right)_{j}$ is diagonal, the quark mass matrices do not fulfill this property, since experiments show that the weak eigenstates of the quarks are not their mass eigenstates. In order to write the Lagrangian in terms of these quark masseigenstates, the matrices have to be diagonalized. This can be done with the help of two unitary matrices, applying the general fact that for every matrix $M$ two unitary matrices $S$ and $T$ exist (fulfilling $S S^{\dagger}=T T^{\dagger}=\mathbb{1}$ ), such that $S^{\dagger} M T=M_{\text {diag. }}$. We can, therefore, rewrite the terms $\bar{u}_{L} M u_{R}$ (and the corresponding one for the down-type states) introducing the mass eigenstates $\hat{u}_{L / R}$ and $\hat{d}_{L / R}$

$$
\begin{aligned}
& \bar{u}_{L} M_{u} u_{R}=\underbrace{\left(\bar{u}_{L} S_{u}\right)}_{\widehat{\hat{u}_{L}}} S_{u}^{\dagger} M_{u} T_{u} \underbrace{\left(T_{u}^{\dagger} u_{R}\right)}_{\hat{u}_{R}}=\overline{\hat{u}_{L}} M_{u, \text { diag. }} \hat{u}_{R} \\
& \bar{d}_{L} M_{d} d_{R}=\underbrace{\left(\bar{d}_{L} S_{d}\right.}_{\widehat{\hat{d}_{L}}}) S_{d}^{\dagger} M_{d} T_{d} \underbrace{\left(T_{d}^{\dagger} d_{R}\right)}_{\hat{d}_{R}}=\overline{\hat{d}_{L}} M_{d, \text { diag. }} . \hat{d}_{R} .
\end{aligned}
$$

However, not both matrices $M_{u}$ and $M_{d}$ can be diagonalized simultaneously, as only three rotations are allowed to be independent. A $3 \times 3$ unitary matrix $V_{\mathrm{CKM}}$, the CabibboKobayashi-Maskawa Matrix, remains to rotate all down-type quarks simultaneously. It appears when we write the charged currents in terms of the observable mass eigenstates, for example

$$
\begin{aligned}
J_{+}^{\mu} & =\sum_{i} \bar{u}_{L i} \gamma^{\mu} d_{L i}+\bar{v}_{L i} \gamma^{\mu} e_{L i} \\
& =\sum_{k l} \overline{\hat{u}}_{L k} \underbrace{\left(S_{u}^{\dagger} S_{d}\right)_{k l}}_{V_{\mathrm{CKM}_{k l}}} \gamma^{\mu} \hat{d}_{L l}+\bar{v}_{L i} \gamma^{\mu} e_{L i} .
\end{aligned}
$$

In this equation, we restrict the rotation to the mass eigenstates to the quark sector. A similar discussion is also valid for the neutrino states, which is not discussed here. The CKM matrix is discussed in the following.

\subsubsection{The Cabibbo-Kobayashi-Maskawa Matrix and the Unitarity Triangle}

The Cabibbo-Kobayashi-Maskawa Matrix is the transformation between the weak eigenstates and the mass eigenstates of the quarks,

$$
\left(\begin{array}{c}
d^{\prime} \\
s^{\prime} \\
b^{\prime}
\end{array}\right)=\left(\begin{array}{lll}
V_{u d} & V_{u s} & V_{u b} \\
V_{c d} & V_{c s} & V_{c b} \\
V_{t d} & V_{t s} & V_{t b}
\end{array}\right) \cdot\left(\begin{array}{c}
d \\
s \\
b
\end{array}\right) .
$$

It is a $3 \times 3$ unitary matrix with nine real parameters. Five of them can be eliminated by phase redefinitions of the quark fields, and the remaining four are three angles and one phase. This phase, if non-zero, gives rise to $C P$ violating interactions in the Standard Model. As the weak interaction connects the up-type with the weak down-type quark in a weak doublet, flavor changing charged currents occur with rates proportional to $\left|V_{i j}\right|^{2}$. 
Given the hierarchy between the CKM matrix elements, it can be parametrized with the four parameters $\lambda, A, \rho$, and $\eta$ in the Wolfenstein parametrization [48],

$$
V_{\mathrm{CKM}}=\left(\begin{array}{ccc}
1-\frac{\lambda^{2}}{2} & \lambda & A \lambda^{3}(\rho-i \eta) \\
-\lambda & 1-\frac{\lambda^{2}}{2} & A \lambda^{2} \\
A \lambda^{3}(1-\rho-i \eta) & -A \lambda^{2} & 1
\end{array}\right)+\mathcal{O}\left(\lambda^{4}\right)
$$

The parameters are defined by the relations,

$$
\lambda=\frac{\left|V_{u s}\right|}{\sqrt{\left|V_{u d}\right|^{2}+\left|V_{u s}\right|^{2}}} \sim 0.22, \quad A \lambda^{2}=\lambda\left|\frac{V_{c b}}{V_{u s}}\right|, \quad A \lambda^{3}(\rho+i \eta)=V_{u b}^{*},
$$

and $\lambda$ is the expansion parameter. The condition of unitarity of the CKM matrix gives rise to three relationships that are relevant for understanding $C P$ violation,

$$
\begin{aligned}
V_{u d} V_{u s}^{*}+V_{c d} V_{c s}^{*}+V_{t d} V_{t s}^{*} & =0 \\
V_{u s} V_{u b}^{*}+V_{c s} V_{c b}^{*}+V_{t s} V_{t b}^{*} & =0 \\
V_{u d} V_{u b}^{*}+V_{c d} V_{c b}^{*}+V_{t d} V_{t b}^{*} & =0 .
\end{aligned}
$$

Each of these equations can be represented geometrically as a unitarity triangle in the complex plane. $C P$ violation manifests itself as a nonzero area of these unitarity triangles. For the first two triangles, one of the sides is much smaller than the other two, resulting in a flattened triangle. This suggests that there are small $C P$ asymmetries in the $K$ system, which is associated with the first triangle, and in $B_{s}$ decays, which are associated with the second triangle. The more similar lengths of the last triangle, referred to as "the Unitarity Triangle", however, suggests that there are large $C P$ asymmetries in $B$-meson decays.

The Unitarity Triangle is depicted in figure 3.1(a). It can be rescaled and rotated, as shown in figure 3.1(b), after choosing a phase convention such that $V_{c d} V_{c b}^{*}$ is real, dividing all the sides by $\left|V_{c d} V_{c b}^{*}\right|$. This aligns one side with the real axis and makes the length of this side one. Then, two of the vertices are fixed at $(0,0)$ and $(0,1)$, and the apex of the triangle is denoted as $(\rho, \eta)$. An expansion of the Wolfenstein parametrization up to $\mathcal{O}\left(\lambda^{6}\right)$ and the introduction of the parameters $\bar{\rho}=\rho\left(1-\lambda^{2} / 2\right)$ and $\bar{\eta}=\eta\left(1-\lambda^{2} / 2\right)$ defines the CKM matrix element $V_{t d}$ as $V_{t d}=A \lambda^{3}(1-\bar{\rho}-\bar{\eta})$. This fixes the apex of the Unitarity Triangle at $(\bar{\rho}, \bar{\eta})$ independent of the phase convention and ensures that the CKM matrix is unitary to all orders in $\lambda$.

Several experimental results can be used to constrain our knowledge of the unitarity triangle [50]. In fact, even such a large amount of measured observables is available that its shape can be overconstrained, thereby testing the consistency of the SM. So far, no significant deviation from the SM has been found.

\subsection{Heavy Quark Effective Theory}

This section will give a short introduction into the concepts of heavy quark physics. However, this review focuses on the description of inclusive semileptonic $B$-meson decays and their observables, useful to extract certain SM parameters and therefore relevant for the presented analysis. 


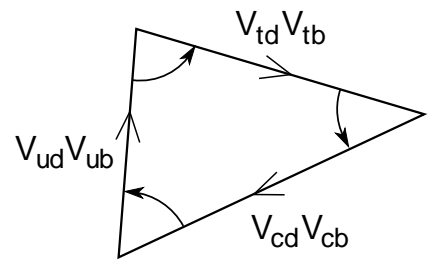

(a)

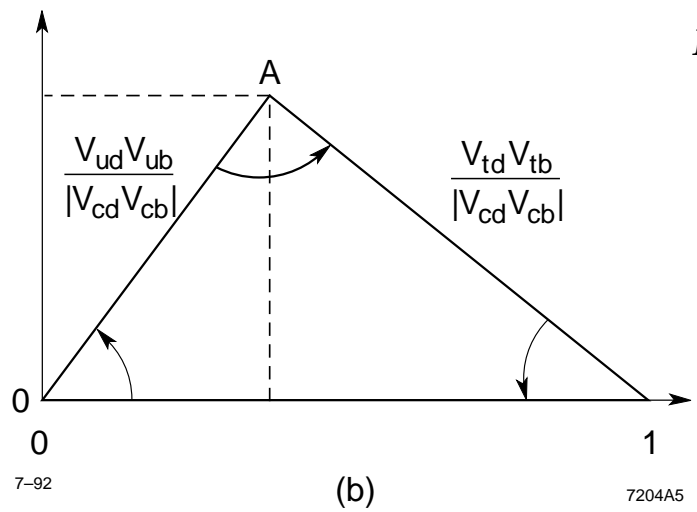

We start with general remarks about the theory describing strong interactions, the quantum chromodynamics (QCD). From QCD, an effective field theory can be derived for the description of $B$-meson physics, using the advantage that heavy degrees of freedom can be identified and exploited, see section 3.2.2. In the framework of this Heavy Quark Effective Theory (HQET), inclusive semileptonic $B$-meson decays can be described using in addition an Operator Product Expansion (OPE), explained in section 3.2.3. Calculations are carried out in different renormalization schemes, among them the so-called kinetic scheme, which is described in further detail in section 3.2.4. This section summarizes information taken from $[51,52]$.

\subsubsection{General Remarks}

In the Standard Model strong interactions are fundamentally described by quantum chromodynamics, an $S U$ (3) gauge group coupling to the color quantum number. Its Lagrangian density is given by

$$
\mathcal{L}_{\mathrm{QCD}}=-\frac{1}{4} G_{a, \mu v} G_{a}^{\mu v}+\sum_{q=\{u, d, s\}} \bar{q}\left(i \not D-m_{q}\right) q+\sum_{Q=\{c, b, t\}} \bar{Q}\left(i \not D-m_{q}\right) Q,
$$

where $q$ and $Q$ are three-component quark fields of the color quantum numbers red, green and blue. The distinction between light $(q)$ and heavy quarks $(Q)$ is at this point not relevant but introduces the basic idea of the following formulation. The $G_{a, \mu \nu}$ are the gluon field strength tensors and the covariant derivative $D$ for the QCD gauge invariance is given by

$$
i D_{\mu}=i \partial_{\mu}-g A_{\mu}^{a} T^{a}=i \partial_{\mu}-g A_{\mu} .
$$


Here $g$ is the strong coupling constant, the $A_{\mu}^{a}$ are the eight gluon gauge fields, and the $T^{a}$ are the eight generators of the gauge group.

Renormalization, that is, removing divergences arising in higher order loop graphs, introduces a logarithmic scale dependence of the coupling constant $g$ and thus $\alpha_{s}=g^{2} /(4 \pi)$. The divergent integrals are usually cut off at a certain scale $\mu$, the renormalization scale of the theory, leading to an effective, scale dependent coupling,

$$
\alpha_{s}(\mu)=\frac{12 \pi}{\left(33-2 N_{q}\right) \ln \left(\mu^{2} / \Lambda_{\mathrm{QCD}}\right)} \quad \text { with } N_{q} \text { the number of quark flavors. }
$$

Thus, $\alpha_{s}$ decreases with increasing energies $\mu$ and formally diverges for small $\mu$. The energy scale where $\alpha_{s}(\mu)$ diverges, i. e. the QCD becomes strongly coupled, defines the scale $\Lambda_{\mathrm{QCD}}$. Here, the perturbation theory breaks down and non-perturbative effects become important. Experimentally it has been found to be of the order of a few hundred $\mathrm{MeV}$, however it does not have an exact value. For the case of $B$ mesons, the difference between the $B$ meson and the $b$ quark is a measure for $\Lambda_{\mathrm{QCD}}$,

$$
\Lambda_{\mathrm{QCD}} \simeq m_{B}-m_{b} \simeq 500 \mathrm{MeV} .
$$

A $B$ meson can be treated as a system containing a heavy quark $Q$ and so-called light degrees of freedom, meaning light quarks and gluons. The mass of the heavy $b$ quark is of the order 4.5 to $5 \mathrm{GeV} / c^{2}$ and thus much larger than $\Lambda_{\mathrm{QCD}}$. Taking this mass scale as the QCD renormalization scale yields $\alpha_{s}\left(m_{b}\right) \sim 0.2$, which shows that perturbation theory is valid at this scale. Furthermore, $\Lambda_{\mathrm{QCD}} / m_{b} \sim 0.1$ can be used as an expansion parameter for non-perturbative effects. These facts are exploited by the Heavy Quark Effective Theory.

An obvious symmetry of the heavy quark limit is the heavy quark flavor symmetry: In the limit of infinite masses, the QCD can not distinguish between different heavy quarks and the dynamics are independent of its flavor. As an interesting consequence, the spectrum of excited heavy hadrons is independent of the flavor of the heavy quark ( [51], p.45). The heavy quark spin symmetry is another symmetry specific to the heavy quark regime, meaning that dynamics are unchanged under arbitrary transformations of the spin of the heavy quark. Together these symmetries form a $U\left(2 N_{\text {heavy }}\right)$ spin-flavor symmetry, which will not be discussed further here.

\subsubsection{From QCD to HQET}

The way to construct an effective theory from QCD to describe heavy quarks is to firstly identify and separate the heavy degrees of freedom.

The momentum exchange between the heavy quark $Q$ and the light degrees of freedom is of order $\Lambda_{\mathrm{QCD}}$, thus, much smaller than $m_{Q}$. This results in the heavy quark being never far from its mass shell, $p_{Q}^{2}=m_{Q}^{2}$. It is convenient to write the momentum $p_{Q}^{\mu}$ as a sum of two parts

$$
p^{\mu}=m_{Q} v^{\mu}+k^{\mu}
$$

Here, $m_{Q} v^{\mu}$ is the large on-shell part with $v^{\mu}$ the four-velocity of the heavy quark. The small fluctuation $k^{\mu} \sim \Lambda_{\mathrm{QCD}}$, originating from the non-perturbative interactions holding 
the hadron together, determines the off-shell size of the heavy quark. The soft interactions inside the meson can change $k^{\mu}$ but not the quarks four-velocity in the heavy quark limit, thus $v^{\mu}$ is found to be a good quantum number of the effective QCD Lagrangian. To separate the heavy and light degrees of freedom, we use this velocity to split the heavy quark field $Q$ into two components

$$
Q_{ \pm}(x)=\frac{1 \pm \psi}{2} Q(x) \quad \text { with } \quad Q=Q_{+}+Q_{-}, \quad \psi Q_{ \pm}= \pm Q_{ \pm}, \quad Q_{ \pm}=\frac{1 \pm \psi}{2} Q_{ \pm} .
$$

If we use these equations to put them in the heavy part of the QCD Lagrangian (eq. (3.14)), we obtain

$$
\mathcal{L}_{Q}=\bar{Q}_{+}\left(i v \cdot D-m_{Q}\right) Q_{+}-\bar{Q}_{-}\left(i v \cdot D-m_{Q}\right) Q_{-}+\bar{Q}_{+}\left(i \not D_{\perp}\right) Q_{-}+\bar{Q}_{-}\left(i \not D_{\perp}\right) Q_{+} .
$$

The subscript $\perp$ defines the perpendicular part of any vector $X^{\mu}$ w. r. t. to the velocity $v$, $X_{\perp}^{\mu}=X^{\mu}-(v \cdot X) v^{\mu}$. Modifying the quark fields $Q_{ \pm}$by a phase $e^{i m_{Q} v \cdot x}$ effectively moves the $m_{Q}$ dependence into the $Q_{-}$part only,

$$
Q_{v}(x)=e^{i m_{Q} v \cdot x} Q_{+}(x) \quad \text { and } \quad \mathcal{Q}_{v}(x)=e^{i m_{Q} v \cdot x} Q_{-}(x) .
$$

The Lagrangian then takes the form (still equivalent to eq. (3.14)):

$$
\mathcal{L}_{Q}=\underbrace{\bar{Q}_{v}(i v \cdot D) Q_{v}}_{\text {massless }}-\underbrace{\overline{\mathcal{Q}}_{v}\left(i v \cdot D+2 m_{Q}\right) \mathcal{Q}_{v}}_{\text {heavy d.o.f with mass } 2 m_{Q}}+\underbrace{\bar{Q}_{v}\left(i \not D_{\perp}\right) \mathcal{Q}_{v}+\overline{\mathcal{Q}}_{-}\left(i \not D_{\perp}\right) Q_{v}}_{\text {coupling }} .
$$

In this formulation we can interpret the field $Q_{v}$ as a massless field describing the excitations due to the residual momentum $k$ of the heavy quark. All heavy degrees of freedom are in contrast described by the field $\mathcal{Q}_{v}$ with mass $2 m_{Q}$, and the last two terms in eq. (3.21) describe the coupling of the states, needed to build physical quarks.

The goal of this algebra, as a reminder, is to identify the impact of the heavy quark on the Lagrangian. It becomes obvious by an expansion of $\mathcal{L}$ in orders of $1 / m_{Q}$. Therefore, we eliminate the massive field $\mathcal{Q}_{v}$ in terms of $Q_{v}$. It is gained from the solution to the equation of motion derived from eq. (3.21),

$$
\left(i v \cdot D+2 m_{Q}\right) \mathcal{Q}_{v}=i \not D_{\perp} Q_{v} \quad \text { and thus } \quad \mathcal{Q}_{v}=\frac{1}{i v \cdot D+2 m_{Q}} i \not D_{\perp} Q_{v} .
$$

Inserting this expression back into eq. (3.21) yields,

$$
\begin{aligned}
\mathcal{L}_{Q} & =\bar{Q}_{v}(i v \cdot D) Q_{v}+\bar{Q}_{v} i \not D_{\perp} \frac{1}{i v \cdot D+2 m_{Q}} i \not D_{\perp} Q_{v} \\
& =\bar{Q}_{v}(i v \cdot D) Q_{v}+\frac{1}{2 m_{Q}} \bar{Q}_{v} i \not D_{\perp} \sum_{n=0}^{\infty}\left(-\frac{i v \cdot D}{2 m_{Q}}\right)^{n} i \not D_{\perp} Q_{v}
\end{aligned}
$$

In the second step, geometric series of the denominator were used to expand it into operators of increasing dimension, which are suppressed by increasing powers of $1 /\left(2 m_{Q}\right)$. This step yields the heavy quark expansion and the explicit heavy quark limit $m_{Q} \rightarrow \infty$ becomes applicable. The HQET Lagrangian schematically can be written as

$$
\mathcal{L}_{\mathrm{HQET}}=\mathcal{L}_{0}+\mathcal{L}_{1}+\mathcal{O}\left(\frac{1}{m_{b}^{2}}\right) .
$$


(a)

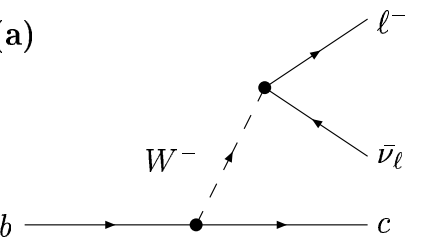

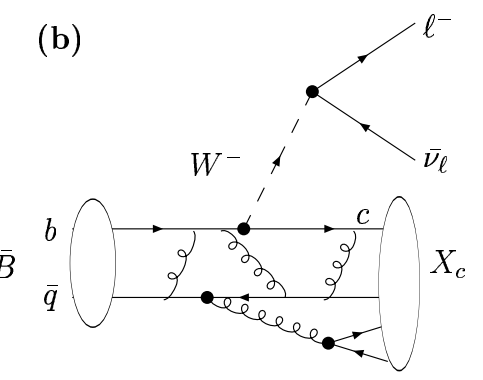

Figure 3.2: Examples of weak decay diagrams for semileptonic $b$ quark (a) and $B$ meson (b) decays. The weak Hamiltonian of the $B$-meson decay can be factored into a leptonic and hadronic current, since leptons do not participate in the strong interaction.

The part of first order in $1 / m_{Q}$ can with some further algebra be rewritten as

$$
\mathcal{L}_{1}=\frac{1}{2 m_{Q}} \bar{Q}_{v}\left(\left(i D_{\perp}\right)^{2}-\frac{g}{2} \sigma \cdot G\right) Q_{v} .
$$

Both terms explicitly break the heavy quark flavor symmetry through the dependence on $m_{Q}$. The first term can be identified as the non-relativistic heavy quark kinetic energy. The second term, due to the spin rotation matrix $\sigma$ also breaks heavy quark spin symmetry and is related to the magnetic moment interaction of the heavy quark.

\subsubsection{Description of Inclusive Semileptonic B-Meson Decays}

This section will derive the theoretical description of semileptonic observables in $B$-meson decays in the framework of a Heavy Quark Effective Theory. As, for this purpose, also an OPE is performed, this formalism is named Heavy Quark Expansion (HQE).

As electromagnetic and strong interactions preserve the quark flavor, the lightest $B$ mesons decays weakly. The simplest approximation of the semileptonic decay of a $B$ meson, $B \rightarrow X_{c} \ell v$, with $X_{c}$ being any hadronic final state containing a charm quark, is to regard the decay of the $b$ quark only, $b \rightarrow c \ell v$ (see figure 3.2). It will be shown that this partonic result is the leading result and thus exact in the limit $m_{b} \rightarrow \infty$. We derive results up to order $1 / m_{b}^{2}$.

Decays of $B$ mesons can be described by the effective weak Hamiltonian density, as the typical energy scale of the process, the $b$-quark mass, is much smaller than the $W$-boson mass,

$$
\begin{aligned}
\mathcal{H}_{W}=\frac{4 G_{F}}{\sqrt{2}} V_{c b}\left(\bar{c} \gamma_{\alpha} P_{L} b\right)\left(\bar{\ell} \gamma^{\alpha} P_{L} v_{\ell}\right) \quad \text { with } \quad P_{L}=\frac{1-\gamma_{5}}{2} \\
\begin{aligned}
\text { and } \quad \bar{c} \gamma_{\alpha} P_{L} b=J_{\alpha(b c)} & \text { the left-handed hadronic current } \\
\text { and } \quad \bar{\ell} \gamma^{\alpha} P_{L} v_{\ell}=J_{(\ell v)}^{\alpha} & \text { the left-handed leptonic current. }
\end{aligned}
\end{aligned}
$$

This Hamiltonian depends explicitly on $V_{c b}$. 
Calculating the rate of semileptonic decays of $B$ mesons to any charm-system uses the expression,

$$
\Gamma=\int \frac{\mathrm{d}^{3} \mathbf{p}_{\ell}}{(2 \pi)^{3} 2 E_{\mathbf{p}_{\ell}}} \int \frac{\mathrm{d}^{3} \mathbf{p}_{v}}{(2 \pi)^{3} 2 E_{\mathbf{p}_{v}}} \frac{1}{2 m_{B}} \sum_{\substack{\text { lepton } \\ \text { spins }}} \sum_{X_{c}}\left|\left\langle X_{c} \ell v\left|\mathcal{H}_{W}\right| \bar{B}\right\rangle\right|^{2}(2 \pi)^{4} \delta^{4}(p_{B}-\underbrace{\left(p_{\ell}+p_{v}\right)}_{=q}-p_{X_{c}}) .
$$

Since the leptons do not interact strongly, it is convenient to factorize the matrix element into two parts, the hadronic one $B \rightarrow W^{*} X_{c}$ and a calculable leptonic part $W^{*} \rightarrow \ell \bar{v}$. This decomposition can be written as,

$$
\begin{aligned}
& \frac{1}{8 m_{b}} \sum_{\substack{\text { lepton } \\
\text { spins }}} \sum_{X_{c}}\left|\left\langle X_{c} \ell v\left|\mathcal{H}_{W}\right| \bar{B}\right\rangle\right|^{2}(2 \pi)^{4} \delta^{4}\left(p_{B}-q-p_{X_{c}}\right) \\
& \quad=2 G_{F}^{2}\left|V_{c b}\right|^{2} W_{\alpha \beta} L^{\alpha \beta}
\end{aligned}
$$

with $W_{\alpha \beta}$ the hadronic tensor and $L^{\alpha \beta}$ the leptonic tensor. The hadronic tensor is defined as,

$$
W_{\alpha \beta}=\sum_{X_{c}} \frac{(2 \pi)^{3}}{2 m_{B}} \delta^{4}\left[p_{B}-q-p_{X_{c}}\right] \cdot\left\langle B\left|J_{(b c)}^{\dagger \alpha}\right| X_{c}\right\rangle\left\langle X_{c}\left|J_{(b c)}^{\beta}\right| B\right\rangle .
$$

It depends on the $B$ four-momentum $p_{B}=m_{b} v$ and the momentum transfer $q$. It can be expanded using an Operator Product Expansion (OPE) in terms of local operators,

$$
W_{\alpha \beta}=\sum_{i} C_{i} O_{i}
$$

where the coefficients can be reliably computed using QCD perturbation theory. Six local operators up to order $\mathcal{O}\left(\Lambda^{2}\right)\left(\Lambda=\Lambda_{\mathrm{QCD}} / m_{b}\right)$ exist. These are

$$
\begin{aligned}
O_{0} & =\bar{b}_{v} b_{v}, & P_{0}^{\alpha} & =\bar{b}_{v} \gamma^{\alpha} \gamma_{5} b_{v}, \\
O_{1}^{\alpha} & =\bar{b}_{v} i \hat{D}^{\mu} b_{v}, & P_{1}^{\alpha \mu} & =\bar{b}_{v} i \hat{D}^{\mu} \gamma^{\alpha} \gamma_{5} b_{v}, \\
O_{2}^{\alpha \mu} & =\bar{b}_{v} i \hat{D}^{\mu} i \hat{D}^{v} b_{v} & P_{2}^{\alpha \mu v} & =\bar{b}_{v} i \hat{D}^{\mu} i \hat{D}^{v} \gamma^{\alpha} \gamma_{5} b_{v},
\end{aligned}
$$

where $b_{v}$ stands for a heavy quark field (named $Q_{v}$ in eq. (3.25)) and $i \hat{D}=i D / m_{b}$. The terms that need to be calculated are,

$$
\left\langle B\left|O_{i}\right| B\right\rangle \quad \text { and } \quad\left\langle B\left|P_{i}\right| B\right\rangle \quad \text { for } i=1,2,3 .
$$

To apply the idea of HQET, also the physical hadron states $B$ have to be replaced by their corresponding HQET states $B_{\infty}$. While these states still contain all interactions of the QCD Lagrangian for the gluons and light quark fields, they only describe interactions by $\mathcal{L}_{0}$ for the heavy quark field. This, in other words, acts as a free quark up to order $1 / m_{Q}$. Details of this expansions will not be shown here, we restrict this section to the discussion of the results and a short outline of the idea to obtain them. 
Calculations reveal that only four of these expressions do not vanish. The elements of $P_{0}^{\alpha}$ and $P_{1}^{\alpha \mu}$ vanish exploiting arguments of parity invariance of the strong interaction. The matrix element containing $O_{1}^{\alpha}$ vanishes in leading order in $m_{b}$ due to the equation of motion, $(i v \cdot D) Q_{v}=0$, which is gained from the leading term $\mathcal{L}_{0}$ in the HQE Lagrangian (cf. eq. (3.23)). However, calculating this matrix element up to $\mathcal{O}\left(1 / m_{b}{ }^{2}\right)$ it is non-zero. The remaining matrix elements can with further algebra be computed and result as,

$$
\begin{aligned}
\frac{1}{2}\left\langle B_{\infty}\left|O_{0}\right| B_{\infty}\right\rangle & =\frac{1}{2}\left\langle B_{\infty}\left|\overline{b_{v}} b_{v}\right| B_{\infty}\right\rangle=1, \\
\frac{1}{2}\left\langle B_{\infty}\left|O_{1}^{\alpha}\right| B_{\infty}\right\rangle & =\frac{1}{2}\left\langle B_{\infty}\left|\overline{b_{v}} i \hat{D}^{\mu} b_{v}\right| B_{\infty}\right\rangle=\frac{\lambda_{1}}{m_{b}^{2}}+\frac{3 \lambda_{2}}{m_{b}^{2}}, \\
\frac{1}{2}\left\langle B_{\infty}\left|O_{2}^{\mu v}\right| B_{\infty}\right\rangle & =\frac{1}{2}\left\langle B_{\infty}\left|\overline{b_{v}} i \hat{D}^{\mu} i \hat{D}^{v} b_{v}\right| B_{\infty}\right\rangle=\frac{\lambda_{1}}{3 m_{b}^{2}}\left(\eta^{\mu v}-v^{\mu} v^{v}\right), \\
\frac{1}{2}\left\langle B_{\infty}\left|P_{2}^{\alpha \mu v}\right| B_{\infty}\right\rangle & =\frac{1}{2}\left\langle B_{\infty}\left|\bar{b}_{v} i \hat{D}^{\mu} i \hat{D}^{v} \gamma^{\alpha} \gamma_{5} b_{v}\right| B_{\infty}\right\rangle=\frac{\lambda_{2}}{6 m_{b}^{2}} d_{H} i \varepsilon_{\lambda}^{\alpha \mu v} v^{\lambda} .
\end{aligned}
$$

In this formulation, the only two appearing parameters $\lambda_{1}$ and $\lambda_{2}$ are defined as,

$$
\begin{aligned}
\lambda_{1} & =\frac{1}{2}\left\langle B_{\infty}\left|\overline{b_{v}}\left(i D_{\perp}\right)^{2} b_{v}\right| B_{\infty}\right\rangle \\
\lambda_{2} d_{H} & =-\frac{g}{4}\left\langle B_{\infty}\left|\overline{b_{v}}(\sigma \cdot G) b_{v}\right| B_{\infty}\right\rangle, \quad \text { with } d_{H} \text { a spin-dependent factor. }
\end{aligned}
$$

Comparing these two matrix-elements to eq. (3.25), we observe that we obtain the matrix elements of the two terms in the Lagrangian $\mathcal{L}_{1}$. The power of HQE really is this small number of parameters that describe any hadronic matrix element of HQET states and QCD operators. This allows to extract them from one observable and use them for the predictions of other observables.

Pushing this formalism forward to order $1 / m_{b}^{3}$, introduces another set of two HQE parameters, or in other words matrix elements of another two local operators, namely

$$
O_{5}^{\mu \nu \lambda}={\overline{b_{v}}}_{i} \hat{D}^{\mu} i \hat{D}^{\lambda} i \hat{D}^{v} b_{v} \quad \text { and } \quad P_{5}^{\alpha \mu \nu \lambda}=\overline{b_{v}} i \hat{D}^{\mu} i \hat{D}^{\lambda} i \hat{D}^{v} \gamma^{\alpha} \gamma_{5} b_{v}
$$

Matrix elements of these operators are further introduced in the following section describing the kinetic scheme.

\subsubsection{The Kinetic Scheme}

For the interpretation of the measured hadronic moments in this thesis calculations in the kinetic scheme [53] are used. The kinetic scheme is a renormalization scheme that defines a so-called kinetic mass $m_{\text {kin }}(\mu)$ from a sum rule for the kinetic energy of the heavy quark and the HQE parameters discussed in the previous section also at its certain renormalization 
point $\mu$. The total semileptonic rate is calculated up to order $1 / m_{b}^{3}$ as [32]

$$
\begin{aligned}
\Gamma_{\mathrm{SL}}\left(B \rightarrow X_{c} \ell v\right) & =\frac{G_{F}^{2} m_{b}^{5}}{192 \pi^{3}}\left|V_{c b}\right|^{2}\left(1+A_{\mathrm{ew}}\right) A_{\text {pert }}(r, \mu) \\
& \times\left[z_{0}(r)\left(1-\frac{\mu_{\pi}^{2}-\mu_{G}^{2}+\frac{\rho_{D}^{3}+\rho_{\mathrm{LS}}^{3}}{c^{2} m_{b}}}{2 c^{4} m_{b}^{2}}\right)\right. \\
& \left.-2(1-r)^{4} \frac{\mu_{G}^{2}+\frac{\rho_{D}^{3}+\rho_{\mathrm{LS}}^{3}}{c^{2} m_{b}}}{c^{4} m_{b}^{2}}+d(r) \frac{\rho_{D}^{3}}{c^{6} m_{b}^{3}}+\mathcal{O}\left(1 / m_{b}^{4}\right)\right],
\end{aligned}
$$

with $r=m_{c}{ }^{2} / m_{b}{ }^{2}$. The tree level phase space factor $z_{0}(r)$ is defined as

$$
z_{0}(r)=1-8 r+8 r^{3}-r^{4}-12 r^{2} \ln r
$$

and the expression $d(r)$ is given by

$$
d(r)=8 \ln r+\frac{34}{3}-\frac{32}{3} r-8 r^{2}+\frac{32}{3} r^{3}-\frac{10}{3} r^{4} .
$$

To account for electroweak corrections, the factor $1+A_{\text {ew }}$ is added to the formula. It can be estimated to be approximately,

$$
1+A_{\mathrm{ew}} \approx\left(1+\frac{\alpha}{\pi} \ln \frac{M_{Z}}{m_{b}}\right)^{2} \approx 1.014 .
$$

The quantity $A_{\text {pert }}$ accounts for perturbative contributions and is approximately $A_{\text {pert }} \approx$ 0.908 . The parameter $\mu$, chosen to be $\mu=1 \mathrm{GeV}$, denotes the renormalization scale that separates effects from long- and short-distance dynamics.

The expression in eq. (3.36) is in leading order equal to the decay to the free $b$ quark. The leading non-perturbative corrections arise at order $1 / m_{b}^{2}$. As discussed above, they are controlled by matrix elements of local operators. In the kinetic scheme, these matrix elements are named $\mu_{\pi}^{2}$ and $\mu_{G}^{2}$, referring to the kinetic and chromomagnetic operators, respectively,

$$
\mu_{\pi}^{2}(\mu) \equiv-\frac{\langle B|\vec{b} \vec{D} b| B\rangle_{\mu}}{2 m_{B}}, \quad \quad \mu_{G}^{2}(\mu) \equiv-\frac{\left\langle B\left|\bar{b} \sigma^{\mu v} G_{\mu \nu} b\right| B\right\rangle_{\mu}}{4 m_{B}}
$$

As outlined above, corrections of order $1 / \mathrm{m}_{b}^{3}$ arise from two additional operators, they are named Darwin and "spin-orbital" $L S$ terms in the kinetic scheme,

$$
\rho_{D}^{3}(\mu) \equiv-\frac{\langle B|\vec{b} \vec{D} \cdot \vec{E} b| B\rangle_{\mu}}{4 m_{B}}, \quad \rho_{\mathrm{LS}}^{3}(\mu) \equiv \frac{\langle B|\vec{b}(\vec{\sigma} \cdot \vec{E} \times i \vec{D}) b| B\rangle_{\mu}}{2 m_{B}}
$$

HQE calculations for moments of various inclusive observables in $B$-meson decays, such as the lepton energy, the hadronic mass, or the hadronic variable $n_{X}^{2}$, as defined in eq. (1.1), rely on the same set of non-perturbative parameters as the total semileptonic rate. Calculations for the moments of the hadronic $n_{X}^{2}$ distribution in decays $B \rightarrow X_{c} \ell v$, are reported 
in [6]. They are given by linearized expressions,

$$
\begin{aligned}
\left\langle n_{X}^{k}\right\rangle\left(m_{b}, m_{c}, \mu_{\pi}^{2}, \mu_{G}^{2}, \rho_{D}^{3}, \rho_{\mathrm{LS}}^{3} ; \alpha_{s}\right)=V+B\left(m_{b}-4.6 \mathrm{GeV}\right)+C\left(m_{c}-1.2 \mathrm{GeV}\right) & \\
& +P\left(\mu_{\pi}^{2}-0.4 \mathrm{GeV}^{2}\right)+D\left(\rho_{D}^{3}-0.1 \mathrm{GeV}^{3}\right) \\
& +G\left(\mu_{G}^{2}-0.35 \mathrm{GeV}^{2}\right)+L\left(\rho_{\mathrm{LS}}^{3}+0.15 \mathrm{GeV}^{3}\right)+S\left(\alpha_{s}-0.22\right),
\end{aligned}
$$

where the dependence on the HQE parameters is described with respect to meaningful reference values. The coefficients are of the dimension of powers of $\mathrm{GeV}$ according to the order $k$ of the calculated moment. Values for these coefficients are given in [6] for discrete values of the minimum lepton momentum $p_{\ell, \mathrm{BRF}}$, however, the calculations have been updated to give more precise predictions as function of $p_{\ell, \mathrm{BRF}}$.

Furthermore, the theoretical calculation of the total semileptonic rate depends on the CKM matrix element $\left|V_{c b}\right|$ and will be used for its extraction in a global fit to the hadronic moments, the $n_{X}^{2}$ moments measured in this analysis and hadronic mass moments, combined with moments of the lepton energy spectrum in semileptonic $B$-meson decays and moments of the photon energy spectrum in decays $B \rightarrow X_{s} \gamma$. Section 8 gives a description of the extraction procedure.

\subsection{Bottom Quark Mass Definitions}

In the framework of $B$ physics, the $b$ quark mass $m_{b}$ is particularly of importance, as theoretical predictions of many quantities depend on it. However, unlike the leptons, quarks are confined inside hadrons and are not observed as physical particles. Thus the quark mass cannot be easily interpreted as a weight or rest mass of a quark. They cannot be measured directly, but must be determined indirectly through their influence on hadron properties. Consequently, the values of the quark masses depend on how they are defined and there is no obvious definition. This has to be kept in mind when speaking of a quark mass, which cannot be referred to in the same sense as the electron or muon mass.

In principle, any renormalization scheme can be used for the definition of quark masses. The difference between two mass schemes can be determined as a series in powers of $\alpha_{s}$. As in most cases, there are schemes that are more appropriate for some purposes than others. The main schemes referred to in semileptonic decays $b \rightarrow c \ell v$ are the so-called kinetic scheme and the so-called $1 \mathrm{~S}$ scheme, which, therefore, are explained in more detail here. We also describe the most commonly used mass, the so-called $\overline{\mathrm{MS}}$ mass, and the pole mass, which is related to the concept of a free quark. This section follows the reports in $[54,55]$.

The Pole Mass The bottom quark pole mass is defined as solution to the equation

$$
\not p-m_{b}-\left.\sum\left(p, m_{b}\right)\right|_{p^{2}=m_{b}^{2}}=0,
$$

where $\sum\left(p, m_{b}\right)$ is the $b$ quark self energy. This equation shows that this pole mass is directly related to the concept of a free quark. It has the disadvantage that it cannot be related precisely to physical quantities due to its strong sensitivity to infrared gluons and uncertainties are hard to estimate. These masses with an ambiguity of order $\Lambda_{\mathrm{QCD}}^{2} / m_{b}$ are generally called short-distance masses. 
The $\overline{\mathrm{MS}}$ Mass The most common short-distance mass parameter is the $\overline{\mathrm{MS}}$ mass $\overline{m_{b}}(\mu)$. It is defined by regulating QCD with dimensional regularization and subtracting the divergences in the $\overline{\mathrm{MS}}$ scheme, a particular renormalization scheme to absorb the divergent part plus a universal constant into the counterterms. By construction, the $\overline{\mathrm{MS}}$ mass is only sensitive to scales of order or larger than $m_{b}$.

The bottom quark $\overline{\mathrm{MS}}$ mass naturally arises in processes where the bottom quark is far off-shell. It is less useful for processes where the bottom quark has non-relativistic energies.

The Kinetic Mass The disadvantages of the $\overline{\mathrm{MS}}$ mass and the pole mass in describing non-relativistic bottom quarks can be resolved by other mass definitions, the so-called threshold masses. There is an infinite number of threshold masses which are defined through subtractions containing contributions which are universal for the dynamics of nonrelativistic quarks. Furthermore they are free of any ambiguity of order $\Lambda_{\mathrm{QCD}}$.

The kinetic mass is one of these threshold masses, and it is defined as

$$
m_{b, \text { kin }}\left(\mu_{\text {kin }}\right)=m_{b, \text { pole }}-\left[\bar{\Lambda}\left(\mu_{\text {kin }}\right)\right]_{\text {pert }}-\left[\frac{\mu_{\pi}^{2}\left(\mu_{\text {kin }}\right)}{2 m_{b, \text { kin }}\left(\mu_{\text {kin }}\right)}\right]_{\text {pert }}+\cdots,
$$

where $\left[\bar{\Lambda}\left(\mu_{\mathrm{kin}}\right)\right]_{\text {pert }}$ and $\left[\mu_{\pi}^{2}\left(\mu_{\text {kin }}\right)\right]_{\text {pert }}$ are perturbative evaluations of HQET matrix elements that describe the difference between the pole and the $B$ meson mass.

The relation between the kinetic mass and the $\overline{\mathrm{MS}}$ mass is known to $\mathcal{O}\left(\alpha_{s}^{2}\right)$ and $\mathcal{O}\left(\alpha_{s}^{2} \beta_{0}\right)$ and can be found in the literature (see references in [55]). Reference masses in the kinetic and the $\overline{\mathrm{MS}}$ scheme are given in [55]. The relationship can be approximated by a linear function in the mass range of interest between $4.1<m_{b}\left(m_{b}\right)_{\overline{\mathrm{MS}}}<4.3 \mathrm{GeV} / c^{2}$. To get a rule of thumb, a linear fit to these reference masses can be performed and yields

$$
m_{b}\left(m_{b}\right)_{\overline{\mathrm{MS}}}=0.191 \mathrm{GeV} / c^{2}+0.876 \cdot m_{b, \mathrm{kin}} .
$$

The 1S Mass The 1S mass is related to a physical quantity. It is defined as half of the perturbative contribution to the mass of a $J^{P C}=1^{--}$and ${ }^{2 s+1} L_{J}={ }^{3} S_{1}$ bound $b \bar{b}$ state, which are the quantum numbers of the $\Upsilon(1 S)$ meson. The $1 \mathrm{~S}$ mass is related to the pole mass utilizing a three loop non-relativistic expansion,

$$
M_{b}^{1 S}=m_{b}^{\text {pole }}\left[1-\varepsilon \Delta_{\mathrm{LO}}\left(\alpha_{s}\right)-\varepsilon^{2} \Delta_{\mathrm{NLO}}\left(m_{b}^{\text {pole }}, \alpha_{s}, \mu\right)-\varepsilon^{3} \Delta_{\mathrm{NNLO}}\left(m_{b}^{\text {pole }}, \alpha_{s}, \mu\right)\right] .
$$

Here, $\alpha_{s}$ is scale dependent $\alpha_{s}(\mu)$. The corrections $\Delta$ can be found in the literature ( [55] and references therein).

The $1 \mathrm{~S}$ mass can also be related to the $\overline{\mathrm{MS}}$ mass. A rule of thumb can be derived using a linear fit to reference masses in the $1 \mathrm{~S}$ and the $\overline{\mathrm{MS}}$ scheme given in [55] between $4.1<m_{b}\left(m_{b}\right)_{\overline{\mathrm{MS}}}<4.3 \mathrm{GeV} / c^{2}$. It results as

$$
m_{b}\left(m_{b}\right)_{\overline{\mathrm{MS}}}=-0.094 \mathrm{GeV} / c^{2}+0.908 \cdot M_{b}^{1 S} .
$$




\section{Outline of the Analysis Strategy}

In this section the presented analysis will be put in the context of other measurements of semileptonic $B$-meson decays aiming at the determination of $\left|V_{c b}\right|$, the quark masses, and the HQE parameters. The general analysis strategy will be outlined.

As discussed in chapter 2, the measurement of inclusive observables in semileptonic decays $B \rightarrow X_{c} \ell v$ have lead to a significant decrease of the uncertainty on $\left|V_{c b}\right|$ and particularly on the quark masses. Lepton-energy moments and hadronic-mass moments have been measured by different experiments and with increased precision ( [4,33,34] and [1-5]). Experimentally the measurement of lepton-energy moments is different from hadronic-mass moments, as the electron and its energy is measured directly while in the latter not necessarily all particles of the hadronic system are detected.

The presented analysis reconstructs $B$ mesons produced in $e^{+} e^{-}$collisions at $10.58 \mathrm{GeV}$. The used technique fully reconstructs one $B$ meson in events $e^{+} e^{-} \rightarrow \Upsilon(4 S) \rightarrow B \bar{B}$ in several hadronic decay modes $\left(B_{\text {reco }}\right)$. Thereby all decay products can be separated and associated with either of the two $B$ mesons. The signal $B$ meson $\left(B_{\mathrm{sl}}\right)$ is identified within the remaining particles, the so-called recoil. This technique is similar to many other analyses performed in BABAR [56-59], which also need a good resolution of their signal decay observables as well as low background and on the other hand can cope with a low overall reconstruction efficiency. The presented analysis describes an inclusive reconstruction, meaning that it does not reconstruct the exclusive signal decays explicitly but sums over all final states containing a charm quark. Therefore, these requirements are crucial.

The $B_{\text {reco }}$ meson is reconstructed semi-exclusively in hadronic decay modes, e.g. $B \rightarrow$ $D^{*} \pi$. The quality of the reconstruction is identified by the two variables $m_{\mathrm{ES}}$ and $\Delta E$ leading to common techniques of choosing one $B_{\text {reco }}$ candidate per event. All remaining charged tracks and neutral particles not used for the $B_{\text {reco }}$ reconstruction and fulfilling dedicated reconstruction requirements, are assumed to belong to the decay chain of the other $B$ meson. Among these tracks exactly one identified electron or muon is required, implying that events with more than one identified lepton are rejected. All remaining tracks and neutral particles are combined to form the hadronic system. Therefore, particles identified as pions, kaons, or protons are assigned the respective mass hypothesis. Neutral candidates are always treated as photons. If the decay really was a semileptonic $B \rightarrow$ $X \ell v$ decay and all particles of the hadronic system were reconstructed, the only missing particle would be the neutrino. From the well known initial state the unmeasured energy and momentum $(P=(E, \vec{p}))$ of the event can be calculated,

$$
P_{\text {miss }}=P_{\Upsilon(4 S)}-P_{B_{\text {reco }}}-P_{\ell}-P_{X_{c}} .
$$

The unmeasured neutrino leads to unmeasured energy $\left(E_{\text {miss }}\right)$ and momentum $\left(\vec{p}_{\text {miss }}\right)$ with the constraint that the variable $E_{\text {miss }}-c\left|\vec{p}_{\text {miss }}\right|$ or $m_{\text {miss }}^{2}=E_{\text {miss }}^{2}-\left(c\left|\vec{p}_{\text {miss }}\right|\right)^{2}$ has values around zero. A kinematic fit using energy and momentum conservation of the whole event as well as a mass hypothesis for the neutrino improves the resolution of the measured hadronic system. 


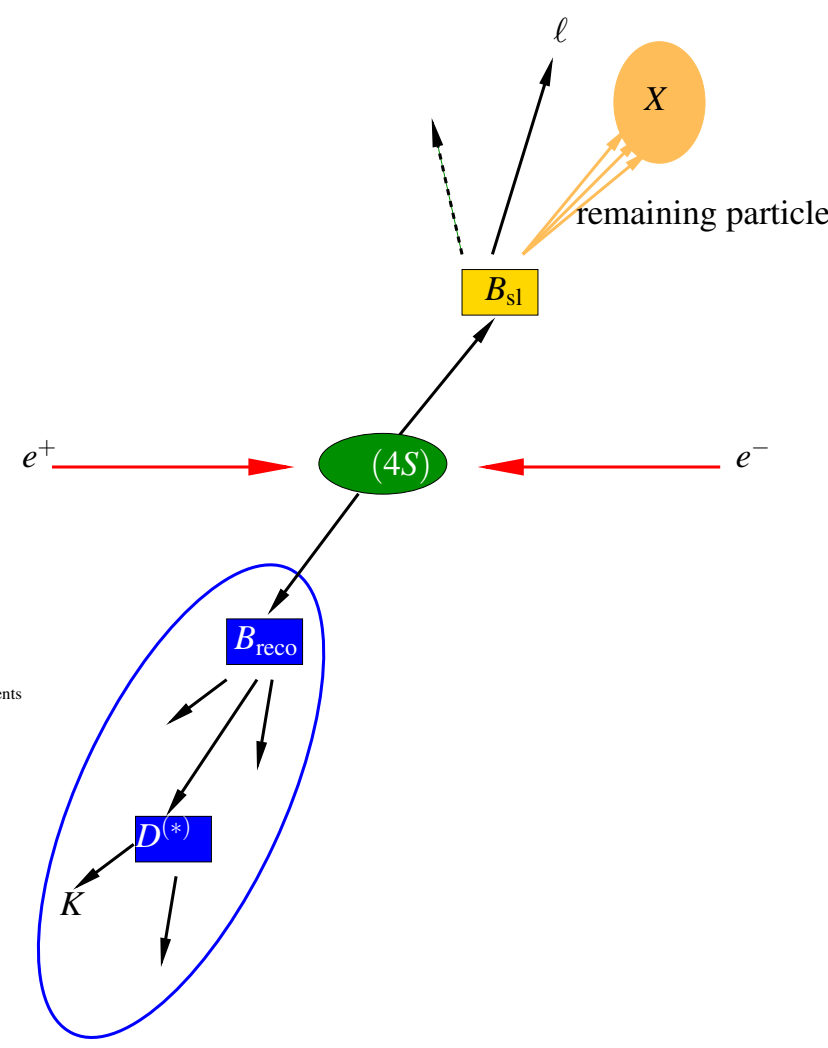

Figure 4.1: Illustration of the reconstructed event structure of the decay $\Upsilon(4 S) \rightarrow B_{\text {reco }} B_{\mathrm{sl}}$. The $B_{\text {reco }}$ (bottom) is reconstructed in a hadronic mode, while the $B_{\mathrm{sl}}$ (top) is identified by a charged lepton (black, solid arrow). The neutrino is not measured (dashed line). All remaining particles are combined to the inclusive $X$ system.

From a theoretical point of view a measurement as inclusive as possible, that is without any cuts on the available phase space, is desirable. However, the lepton identification is only reliable above a certain momentum threshold and less leptons from background processes exist at higher momenta. Thus, the analysis is performed with different lower cuts on the lepton momentum in the $B$ meson rest-frame (BRF) between $p_{\ell, \mathrm{BRF}} \geq 0.8 \mathrm{GeV} / c$ and $p_{\ell, \mathrm{BRF}} \geq 1.9 \mathrm{GeV} / c$. As this construction leads to overlapping data samples, correlations between the measured moments have to be determined and included in the fit. Theory fortunately is able to predict also the dependence of the measured moments on this lower lepton momentum cut.

Background can be reduced by cutting on dedicated event variables such as the flavor and charge correlation between the $B_{\text {reco }}$ and the identified lepton. Further cuts are applied to ensure a good resolution of the measured inclusive system. The shape and amount of the remaining background is determined on data itself if possible and on MC simulations, e. g. for decays $B \rightarrow X_{u} \ell v$ rather than $B \rightarrow X_{c} \ell v$, and subtracted by an event-weighting technique. This method ensures the determination of moments from decays $B \rightarrow X_{c} \ell v$ only.

Even though the kinematic fit already resolves a great part of the distorted $X$ system, Monte Carlo studies still show a significant bias of about $20 \%$ between the reconstructed and the true moments $\left\langle n_{X}^{k}\right\rangle$. This fact is mainly due to missing particles resulting from the limited detector acceptance and to a small amount due to energy misreconstruction. Therefore, a so-called calibration method is implemented which corrects the reconstructed 
moments to the true moments applying linear correction functions event-by-event,

$$
n_{X, \mathrm{calib}}^{k}=\frac{n_{X, \mathrm{reco}}^{k}-p_{0, k}}{p_{1, k}}
$$

where $p_{0, k}$ and $p_{1, k}$ are the parameters of the linear correction functions, which are derived from MC simulations. This technique has been used in previous mass-moments measurements $[2,60,61]$ and has been found to serve a robust and well controllable way to determine the moments of a hadronic spectrum. Even though this method reduces the bias remarkably, it cannot reproduce the initial true moments perfectly. A small bias of the order of a few percent remains after the calibration which is studied in detail on Monte Carlo simulations. Finally, the moments of the hadronic $n_{X}^{2}$ spectrum are calculated by weighted means

$$
\left\langle n_{X}^{k}\right\rangle=\mathcal{C}\left[\frac{1}{\sum_{i=1}^{N_{\mathrm{ev}}} w_{i}\left(n_{X}^{2}\right)} \sum_{i=1}^{N_{\mathrm{ev}}} w_{i}\left(n_{X, i}^{2}\right) \cdot n_{X, \mathrm{calib}, i}^{k}\right] .
$$

The weights $w_{i}\left(n_{X}^{2}\right)$ are the background subtraction factors and $n_{X, \text { calib }}^{k}$ is the corrected value of the reconstructed $n_{X, \text { reco }}^{k}$, determined according to eq. (4.2). The factor $\mathcal{C}$ corrects the small bias remaining after calibration.

The following chapters will lead through each step of the analysis, starting from the description of the $B A B A R$ experiment, describing briefly the $B_{\text {reco }}$ reconstruction, and then turning to the moments measurement. Several verification procedures will be described and the evaluation of systematic uncertainties is discussed. The fit of the theory predictions to the measured moments will be described at the end of this thesis. 



\section{The BABAR Experiment}

The presented analysis uses data collected with the BABAR detector operating at the PEPII $e^{+} e^{-}$-storage rings at the Stanford Linear Accelerator Center (SLAC). This experiment was built as a high luminosity $B$-meson factory with the primary goal to measure timedependent $C P$ asymmetries in decays of neutral $B$ mesons. In addition, the large number of produced $B$ mesons allows the precise determination of CKM-matrix elements.

This chapter gives a brief overview of the concept and the performance of the accelerator and the detector. A more detailed description can be found in [62] for the accelerator and [63] describing the $B A B A R$ detector.

\subsection{The PEP-II B-Meson Factory}

The PEP-II $B$-meson factory [62] was designed to deliver $e^{+} e^{-}$collisions with high luminosity to the $B A B A R$ experiment. It consists of a high energy electron storage ring (HER) with $E_{e^{-}}=9.0 \mathrm{GeV}$ and a low energy positron storage ring (LER) with $E_{e^{+}}=3.1 \mathrm{GeV}$ resulting in an interaction energy in the center-of-mass frame of $10.58 \mathrm{GeV}$ corresponding to the mass of the $\Upsilon(4 S)$ resonance. The layout is shown in figure 5.1. Since the mass of the bound $b \bar{b}$ state is about $20 \mathrm{MeV}$ above the production threshold of $B$-meson pairs, the $\Upsilon(4 S)$ resonance decays predominantly into two $B$ mesons with a branching fraction larger than $96 \%$ [42]. The asymmetric energies of the two beams result in a boost of produced particles of $\beta \gamma=0.55$ with respect to the laboratory frame. This allows a separation of the two $B$-meson decay vertices and thus a measurement of their decay time difference, which is crucial for the measurement of time dependent $C P$ asymmetries.

Between May 1999 and beginning of September 2007 PEP-II delivered $496 \mathrm{fb}^{-1}$ to the BABAR experiment, which were recorded with about $96 \%$ mean efficiency, yielding a recorded luminosity of $477 \mathrm{fb}^{-1}$. This period is divided in six run periods, named Run 1 to Run 6. The peak luminosity of $12.1 \cdot 10^{33} \mathrm{~cm}^{-2} \mathrm{~s}^{-1}$ was achieved in August 2006. Figure 5.2 shows the recorded luminosity over the time.

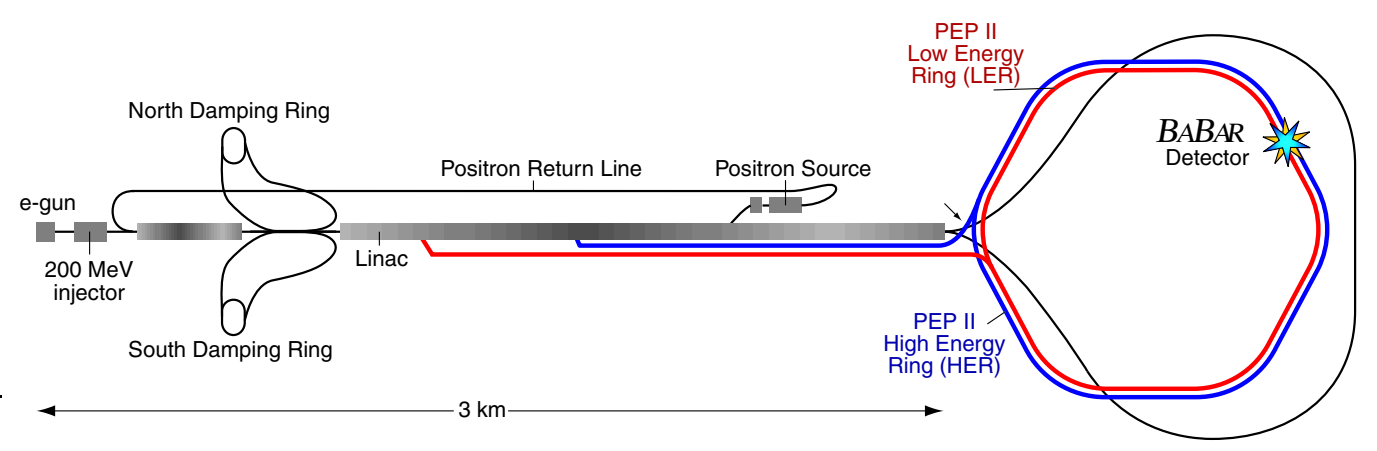

Figure 5.1: The Stanford Linear Collider and the PEP-II $e^{+} e^{-}$-storage rings. 


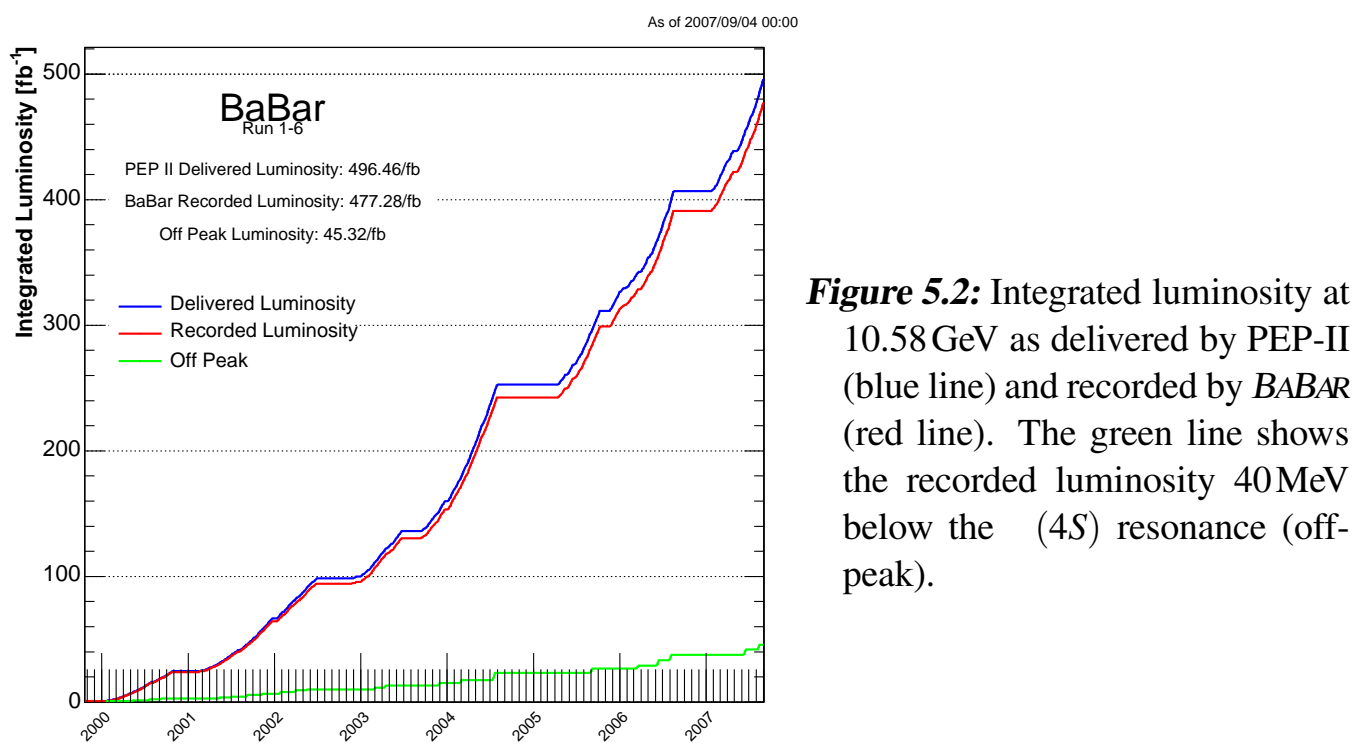

\subsection{The BABAR Detector}

The $B A B A R$ detector is designed as a general-purpose detector capable to reconstruct decay vertices, charged particles' trajectories and energies of neutral particles with high precision and furthermore to yield an excellent separation of particle types. A schematic view of the detector is shown in figure 5.3. Starting from the beam pipe it consists of a Silicon Vertex Tracker (SVT), a cylindrical Drift Chamber (DCH), a Detector of Internally Reflected Cherenkov Light (DIRC), and an Electromagnetic Calorimeter (EMC). These components are embedded in a magnetic field of $1.5 \mathrm{~T}$ delivered by a superconducting solenoid. The Iron Yoke to return the magnetic flux is instrumented to detect muons (IFR).

The right-handed coordinate system used within the BABAR collaboration is defined as follows. The $z$ axis points in direction of the $e^{-}$beam (HER) parallel to the magnetic field, the $y$ axis points vertically upwards. The $x$ axis is directed horizontally away from the center of the PEP-II rings. If cylindrical or spherical coordinates are used, the angle $\theta$ counts the angle to the positive $z$ axis, while $\varphi$ is defined in the $x-y$ plane with $\varphi=0$ in direction of the positive $x$ axis.

As an inclusive analysis, which does not reconstruct final states explicitly, the presented analysis depends crucially on

- a large geometrical acceptance to loose as few particles as possible in the reconstruction,

- the particle identification to distinguish between electrons, muons, pions, kaons, and protons,

- and a good energy and momentum reconstruction to measure the total energy and momentum of the inclusive system as precisely as possible from the sum of the particles' individual four-momenta. 


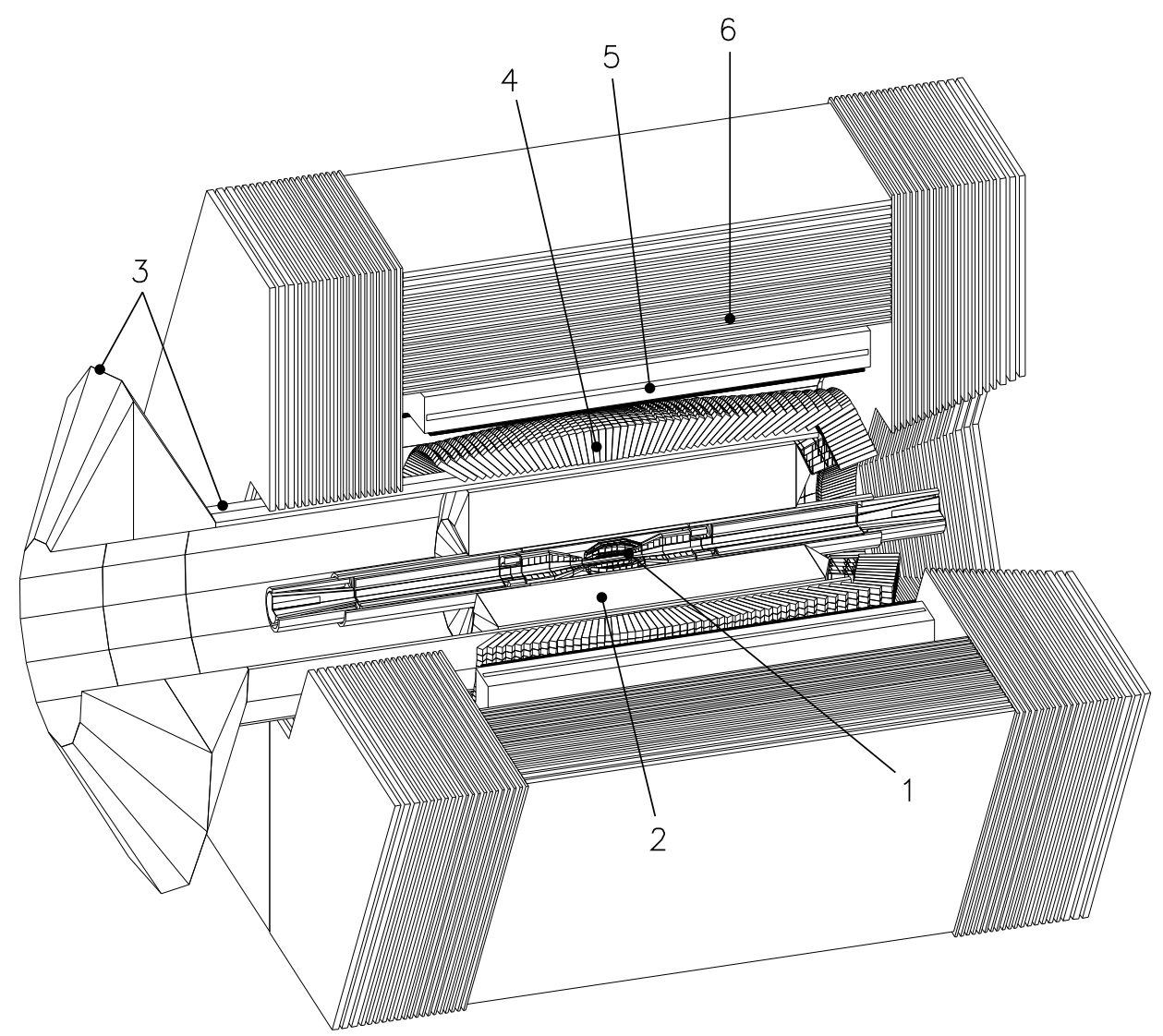

Figure 5.3: Layout of the BABAR detector with its sub-detectors: (1) SVT, (2) DCH, (3) DIRC, (4) EMC, (5) Solenoid Coil, (6) IFR. 


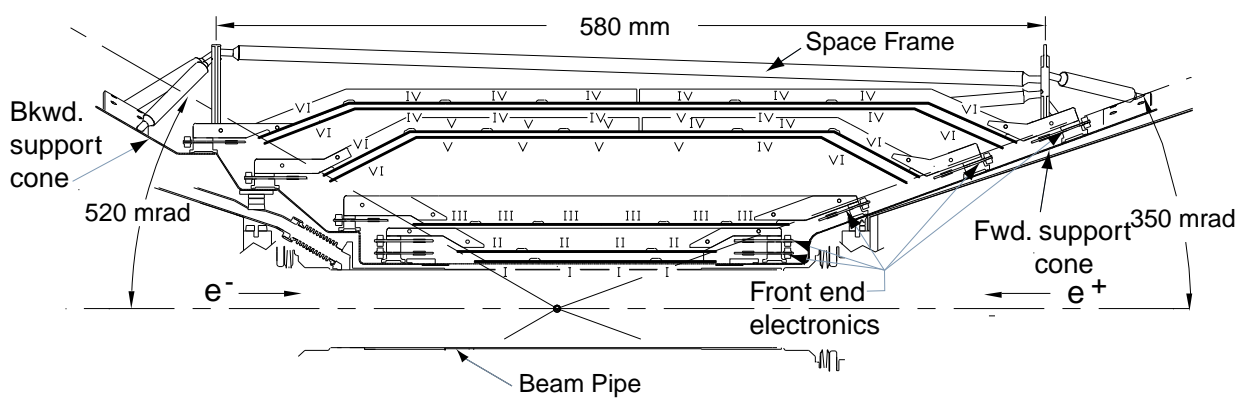

Figure 5.4: Schematic view of SVT: longitudinal section. The roman numerals label the six different types of sensors. The innermost (outermost) layer is located at a distance of $32 \mathrm{~mm}(114-144 \mathrm{~mm})$ from the beam. Picture taken from [63].

These requirements are fulfilled by several detector components which, therefore, are described in more detail in the following.

\subsubsection{Silicon Vertex Tracker}

The reconstruction of $B$-meson vertices is crucial for several analyses performed by the $B A B A R$ collaboration. Therefore, the SVT has been designed to provide precise reconstruction of charged particle trajectories and decay vertices near the interaction region. Especially for particles with low transverse momentum of less than $120 \mathrm{MeV} / c$, the SVT is the only component to yield tracking information. This is important for the slow pions originating from a $D^{* \pm}$ decay. Thus, also for the presented inclusive analysis it is useful to include the measurement of the so-called SVT-only tracks. The spatial resolution for perpendicular tracks, required by various analyses, is $10-15 \mu \mathrm{m}$ in the three inner layers and about $40 \mu \mathrm{m}$ in the two outer layers.

The SVT is composed of five concentric double-sided layers of silicon strip-sensors organized in 6,6,6,16, and 18 modules, respectively. An arch design of layer 4 and 5 was chosen to minimize the amount of silicon required to cover the solid angle and to increase the crossing angle for particles near the edges of acceptance. A schematic view of the SVT giving further geometrical information is shown in figure 5.4.

\subsubsection{Drift Chamber}

The principal purpose of the drift chamber $(\mathrm{DCH})$ is the efficient detection of charged particles and the measurement of their momenta and angles with high precision. For low momentum particles, the DCH is required to provide particle identification by the measurement of their ionization loss $(\mathrm{d} E / \mathrm{d} x)$. A resolution of about $7 \%$ allows $\pi / K$ separation up to $700 \mathrm{MeV} / \mathrm{c}$. This capability is complementary to that of the DIRC in the barrel region, while in the extreme backward and forward directions, the DCH is the only device providing some discrimination of particles of different mass. For high momentum 


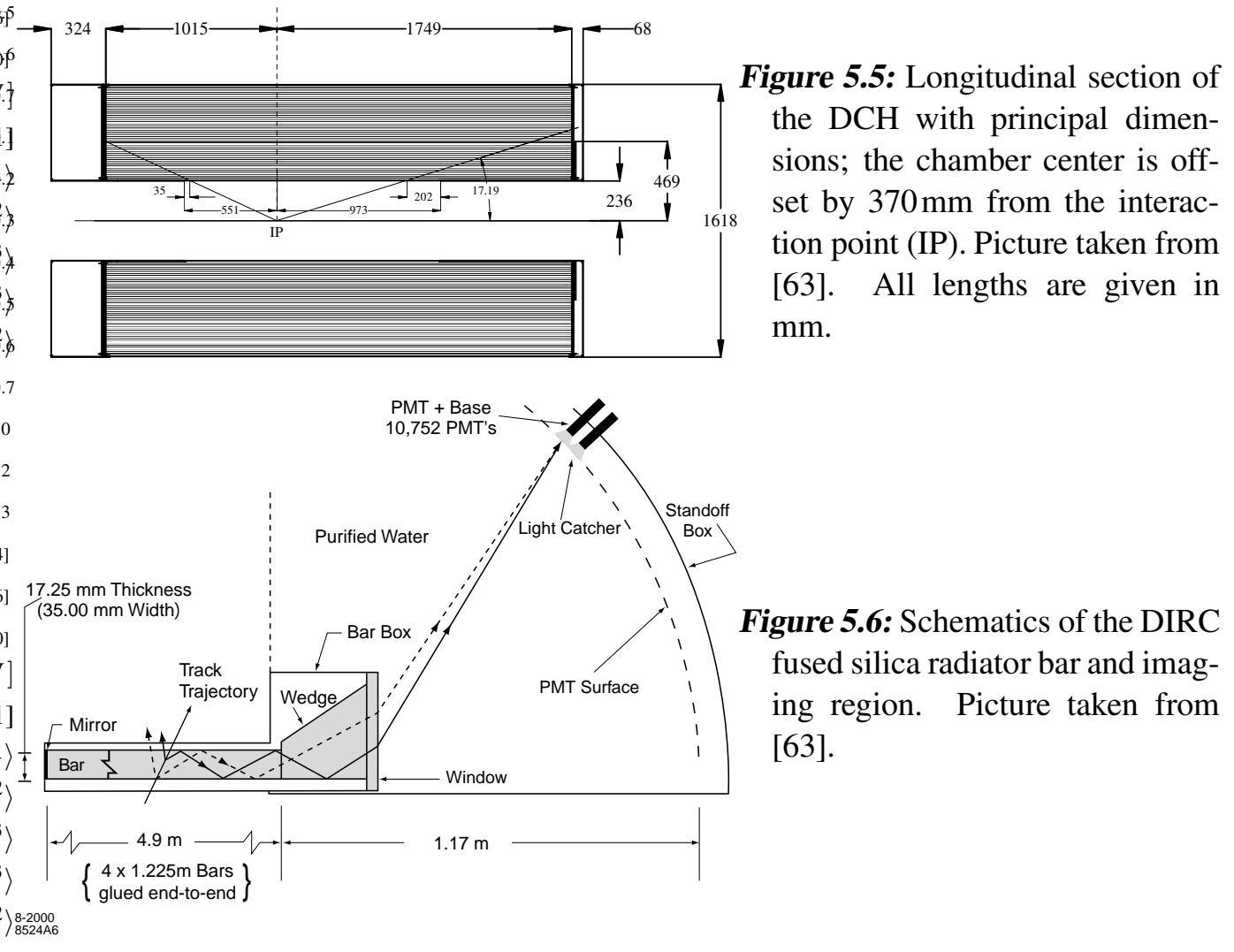

tracks above $1 \mathrm{GeV} / c$, the $\mathrm{DCH}$ yields excellent momentum resolution of approximately $\sigma_{p_{t}} \approx 0.3 \% \cdot p_{t}[49]$.

The layout with further geometrical information is shown in figure 5.5.

\subsubsection{Cherenkov Detector}

The Detector of Internally Reflected Cherenkov light completes the particle identification system of the BABAR detector. It is able to provide $\pi / K$ separation of $\sim 4 \sigma$ or greater for all tracks from $B$-meson decays - from the pion Cherenkov threshold up to momenta of $4.2 \mathrm{GeV} / c$.

The radiator material of the DIRC is synthetic, fused silica in the form of 144 bars ( $4.9 \mathrm{~m}$ long) with a rectangular cross section of $17 \times 35 \mathrm{~mm}^{2}$. These bars serve both as radiators and as light pipes for the portion of the light trapped in the radiator by total internal reflection. The photons are guided into a water-filled expansion region, called the standoff box. They are detected by an array of photomultiplier tubes (PMTs), each surrounded by reflecting light catcher cones to capture light which would otherwise miss the active area of the PMT. A schematic view of the DIRC design is shown in figure 5.6.

\subsubsection{Electromagnetic Calorimeter}

The electromagnetic calorimeter is designed to measure electromagnetic showers with excellent efficiency and energy and angular resolution over the energy range from $20 \mathrm{MeV}$ to 


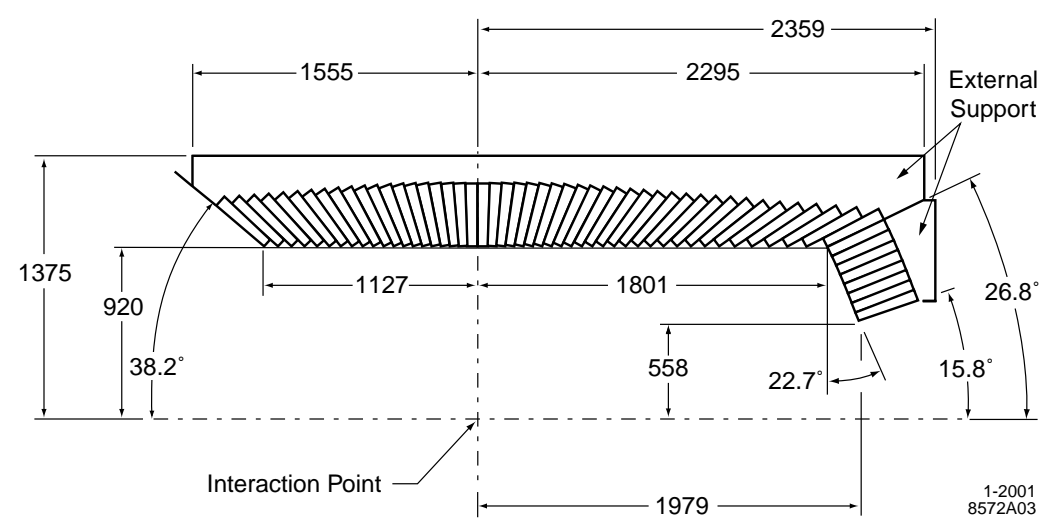

Figure 5.7: A longitudinal cross section of the EMC (only the top half is shown) indicating the arrangement of the 56 crystal rings. The detector is axially symmetric around the zaxis. Picture taken from [63].All lengths are given in $\mathrm{mm}$.

$9 \mathrm{GeV}$. This capability allows the detection of photons from $\pi^{0}$ and $\eta$ decays as well as from electromagnetic and radiative processes. It also yields identification information for electrons which is combined with momentum information from the DCH and the Cherenkov angle from the DIRC in a likelihood discriminant to yield the excellent particle identification of the $B A B A R$ experiment.

Figure 5.7 shows the geometrical arrangement of the thallium-doped cesium iodide (CsI(Tl)) crystals building a segmented calorimeter. Their length varies from $29.6 \mathrm{~cm}$ in the backward region to $32.4 \mathrm{~cm}$ in the forward direction and their cross section in the front is typically $4.7 \times 4.7 \mathrm{~cm}^{2}$, which is comparable to the Molière radius of CsI(Tl) of $3.8 \mathrm{~cm}$. The crystals are arranged in a cylindrical barrel and a conical forward endcap. Thereby, the EMC has full coverage in azimuth angle and extends in polar angle from $16^{\circ}$ to $142^{\circ}$ corresponding to a solid-angle coverage of $90 \%$ in the c.m. system. The barrel contains 5760 crystals arranged in 48 rings with 120 identical crystals each. The endcap holds 820 crystals arranged in eight rings.

The energy and angular resolution can empirically be expressed as

$$
\frac{\sigma_{E}}{E}=\frac{a}{\sqrt[4]{E[\mathrm{GeV}]}} \oplus b \quad \text { and } \quad \sigma_{\theta}=\sigma_{\phi}=\left(\frac{c}{\sqrt{E[\mathrm{GeV}]}}+d\right),
$$

with constants $a, b, c$, and $d$. Values for $a$ and $b$ close to $2 \%$ are obtained by the BABAR calorimeter [63]. An angular resolution of a few mrad is obtained by measured values of $c \approx 3.9 \mathrm{mrad}$ and $d \approx 0$, which are even better than expected from a MC simulation [63].

As an electromagnetic or hadronic shower distributes its energy over several crystals, a so-called cluster algorithm tries to recombine several crystals in order to recover the total energy of a shower. It starts with a crystal with an energy above $10 \mathrm{MeV}$. Surrounding crystals are considered to be part of the same cluster if their energies exceed a threshold of $1 \mathrm{MeV}$ or if they are contiguous neighbors of crystals with at least $3 \mathrm{MeV}$. Clusters containing more than one local energy maximum are split into several so-called bumps. Each cluster or bump is tried to match to a charged track using tracking information obtained by 
the SVT and DCH. If this matching is not successful, a neutral candidate is created with the measured energy and shower shape.

\subsubsection{Instrumented Flux Return}

The Instrumented Flux Return (IFR) was designed to identify muons with high efficiency and good purity, and to detect neutral hadrons, primarily $K_{L}^{0}$ and neutrons, over a wide range of momenta and angles. Especially the muon measurement is important for the identification of a semileptonic decay in the presented analysis.

The IFR uses the steel flux return of the magnet as a muon filter and hadron absorber. Single gap resistive plate chambers (RPCs) have been chosen as detectors [64]. The 774 RPC modules are installed in the gaps of the segmented steel of the barrel (342 modules) and the end doors (432 modules) of the flux return. The steel is segmented into 18 plates, increasing in thickness from $2 \mathrm{~cm}$ for the inner nine plates to $10 \mathrm{~cm}$ for the outermost plates, leaving gaps of 3.2 to $3.5 \mathrm{~cm}$ for the RPCs. They measure streamers from ionizing particles via capacitive readout strips, covering polar angles between $17^{\circ}$ and $157^{\circ}$. Their active volumes are filled with a mixture of Argon (57\%), 39\% Freon 134a (1,1,1,2 tetrafluoroethane) and about $5 \%$ isobutane.

Overall muon identification efficiencies between $65 \%$ and $80 \%$ are achieved for momenta above $1.5 \mathrm{GeV} / c$. The misidentification rates for pions range between 2 and $4 \%$ while they are much lower (1\% or less) for kaons and protons [65], see section 6.3.3 for details.

Due to problems with the linseed oil used in the manufacturing of the RPCs they have lost detection efficiency continuously and much faster than expected. Therefore the RPCs have been replaces in two steps by Limited Streamer Tubes (LSTs) [66]. Between August and September 2004 the barrel's top and bottom sextants have been replaced, and during the shutdown between Run 5 and Run 6 (August 2006 to January 2007) the remaining barrel sextants have been replaced. However, all data used in this analysis was taken before the LST installation. 



\section{Measurement of Moments of the Combined Mass-Energy Spectrum}

This chapter describes the measurement of the hadronic moments $\left\langle n_{X}^{k}\right\rangle, k=2,4,6$. At first, datasets used in this measurement and basic reconstruction criteria are introduced. Reconstruction principles of the $B_{\text {reco }}$ and the signal $B$ meson and event selection criteria are discussed. Background contributions are extracted both from the data and from MC simulations as discussed in this chapter. Then, some studies performed on MC simulations are described. They give insight into the main reasons for the misreconstruction and distortion of the hadronic system and yield tools to correct them. These tools are in turn verified on independent $\mathrm{MC}$ datasets to give confidence in the robustness of the moment extraction procedure. Afterwards, the moment measurement on data is described.

\subsection{Monte Carlo and BABAR Datasets}

For the extraction of the moments the BABAR datasets of the runperiods 1 to 4 are used, recorded between January 2000 and July 2004. They correspond to a recorded luminosity of $210.4 \mathrm{fb}^{-1}$ or about 232 million $B \bar{B}$ pairs. The number of $B \bar{B}$ events is counted centrally by the $B A B A R$ collaboration by counting hadronic events on the $\Upsilon(4 S)$ resonance with dedicated trigger requirements and subtracting continuum contributions from the off-peak dataset recorded $40 \mathrm{MeV}$ below the $\Upsilon(4 S)$ resonance [67].

In addition, three different Monte Carlo datasets of simulated $B \bar{B}$ decays are used in the analysis. All datasets use the event generator EvtGen [68] for the simulation of $B$ meson decays. If included, the detector response to each event is simulated using GEANT4 [69]. Final state radiation (FSR) is modeled with PHOTOS [70]. Eight individual decay modes $B \rightarrow X_{c} \ell v$ are simulated in all datasets. Their branching fractions are rescaled, applying decay mode dependent event weights, to match the latest measurements recommended by the BABAR semileptonic analysis working group (AWG) [71]. These scaling factors are summarized in table 6.1. The following datasets are used:

1. The simplest simulated dataset is pure generator Monte Carlo, which can be produced easily and fast. The GEANT4 detector simulation is switched off, thus this dataset contains only so-called truth information. Both $B$ mesons decay semileptonically into final states with a charm quark, $B \rightarrow X_{c} \ell v$. The simulation of FSR can easily be turned on and off, respectively. Both configurations are produced to study the impact of FSR photons.

2. Another MC dataset is the generic $B \bar{B}$ Monte Carlo, where both $B$ mesons decay into all known final states with the branching fractions measured so far [42]. The full detector response is simulated and reconstructed variables are available. Final state radiation is simulated using PHOTOS. This dataset is most realistically describing the data. 
Table 6.1: Summary of $b \rightarrow c \ell v$ branching fractions and scaling factors in MC quoted separately for $B^{0}$ and $B^{ \pm}$. The original branching fractions in MC [68] are the same for neutral and charged $B$ mesons. The reweighted branching fractions are taken as proposed in [71]. The errors on these branching fractions become relevant for systematic studies discussed in section 7.1.8.

\begin{tabular}{llllll}
\hline $\begin{array}{l}\text { decay } \\
\text { mode }\end{array}$ & $\begin{array}{l}\text { initial } \mathcal{B} \\
{[\%]}\end{array}$ & $\begin{array}{l}\text { meas. } \mathcal{B} \\
{[\%] B^{0}}\end{array}$ & $\begin{array}{l}\text { scale } \\
\text { factor } B^{0}\end{array}$ & $\begin{array}{l}\text { meas. } \mathcal{B} \\
{[\%] B^{ \pm}}\end{array}$ & $\begin{array}{l}\text { scale } \\
\text { factor } B^{ \pm}\end{array}$ \\
\hline$B \rightarrow D \ell v$ & 2.10 & $2.13 \pm 0.14$ & 1.01 & $2.30 \pm 0.16$ & 1.10 \\
$B \rightarrow D^{*} \ell v$ & 5.60 & $5.53 \pm 0.25$ & 0.99 & $5.95 \pm 0.24$ & 1.06 \\
\hline$B \rightarrow D_{1} \ell v$ (narrow) & 0.56 & $0.50 \pm 0.08$ & 0.89 & $0.54 \pm 0.06$ & 0.96 \\
$B \rightarrow D_{2}^{*} \ell v$ (narrow) & 0.37 & $0.39 \pm 0.07$ & 1.05 & $0.42 \pm 0.08$ & 1.14 \\
$B \rightarrow D_{0}^{*} \ell v$ (broad) & 0.20 & $0.43 \pm 0.09$ & 2.15 & $0.45 \pm 0.09$ & 2.25 \\
$B \rightarrow D_{1}^{\prime} \ell v$ (broad) & 0.37 & $0.40 \pm 0.20$ & 1.08 & $0.45 \pm 0.20$ & 1.22 \\
\hline$B \rightarrow D^{0} \pi \ell v$ & 0.60 & $0.40 \pm 0.12$ & 0.67 & $0.20 \pm 0.06$ & 0.33 \\
$B \rightarrow D^{ \pm} \pi \ell v$ & 0.30 & $0.19 \pm 0.06$ & 0.63 & $0.40 \pm 0.12$ & 1.33 \\
$B \rightarrow D^{* 0} \pi \ell v$ & 0.20 & $0.12 \pm 0.04$ & 0.60 & $0.06 \pm 0.02$ & 0.30 \\
$B \rightarrow D^{* \pm} \pi \ell v$ & 0.10 & $0.06 \pm 0.04$ & 0.60 & $0.12 \pm 0.04$ & 1.20 \\
\hline sum & 10.40 & 10.15 & & 10.89 & \\
\hline
\end{tabular}

3. A special MC dataset, named Cocktail $M C$ is produced by the BABAR collaboration. Here one of the $B$ mesons decays in very pure hadronic modes, $\bar{B}^{0} \rightarrow D^{(*)+}$ $\left(\pi^{-} / \rho^{-} / a_{1}^{-}\right)$and $B^{-} \rightarrow D^{(*) 0} \pi^{+}$. The other $B$ meson decays generically (as in the generic dataset) into all final states currently known. The detector and FSR is modeled as in the generic MC. This sample provides a large sample of fully reconstructed $B_{\text {reco }}$ mesons with very low combinatorial background.

The numbers of produced events, divided into charged and neutral $B$ mesons, for each of the datasets are summarized in table 6.2. The configuration of the event generation and background simulation has changed for the simulation of Run 123 and Run 4 data, respectively. The two simulation production cycles are named SP5 for Run 123 and SP6 for Run 4. While input values from physics, such as branching fractions and masses and widths of resonances, such as the orbitally excited $D^{* *}$ mesons, have only changed within their uncertainties, background conditions have changed substantially from Run 123 to Run 4 with increasing luminosity. To take care of these differences, scaling factors also given in table 6.2 are applied as event weights to scale the MC datasets to the ratios of charged and neutral $B$ mesons (mainly important for Cocktail MC, as these numbers differ significantly here) and the number of $B$ mesons in Run 123 and Run 4 in data.

\subsection{Reconstruction and Selection of the $B_{\text {reco }}$ Candidate}

The following section gives an overview of the reconstruction of the $B_{\text {reco }}$ candidate in hadronic decay modes. The reconstruction is done centrally by the BABAR collaboration during one step of the reprocessing of the data, called skimming. The user is provided 
Table 6.2: Summary of datasets used in the analysis. Given is the overall number of decays $\Upsilon(4 S) \rightarrow B \bar{B}$. The weight factors are applied to match the ratios of $B^{+} B^{-}$and $B^{0} \bar{B}^{0}$ simulated for Run 123 and Run 4 to the numbers measured in data. They are calculated from the number of $B \bar{B}$ events and then rescaled to be close to one.

\begin{tabular}{lrrr}
\hline Dataset & $N_{B \bar{B}}$ & \multicolumn{2}{c}{ weight factor } \\
& & Run 123 & Run4 \\
\hline Data & $(231.6 \pm 2.5) \times 10^{6}$ & 1.00 & 1.00 \\
Generic MC $\left(B^{0} \bar{B}^{0}\right)$ & $546.50 \times 10^{6}$ & 0.87 & 0.48 \\
Generic MC $\left(B^{+} B^{-}\right)$ & $544.52 \times 10^{6}$ & 0.87 & 0.48 \\
Cocktail MC $\left(B^{0} \bar{B}^{0}\right)$ & $26.49 \times 10^{6}$ & 0.31 & 0.16 \\
Cocktail MC $\left(B^{+} B^{-}\right)$ & $6.28 \times 10^{6}$ & 0.88 & 0.96 \\
Generator MC & $37.97 \times 10^{6}$ & & \\
\hline
\end{tabular}

with a dataset (called BSemiExcl skim), in which candidates of fully reconstructed $B$ meson candidates are stored [72]. The user has to select one of the candidates with criteria suitable for the special analysis and perform the analysis with the rest of the event. Events in which only badly reconstructed $B_{\text {reco }}$ candidates are found, can be rejected by the user.

\subsubsection{Kinematic Variables}

Within the $B A B A R$ collaboration two variables are commonly used to identify fully reconstructed $B$ mesons. These are the energy difference $\Delta E$ and the energy-substituted mass $m_{\mathrm{ES}}$. The two variables are constructed to be minimally correlated [73]. The energy difference is defined as

$$
\Delta E=E_{B_{\text {reco }}}^{*}-E_{\text {beam }}^{*}
$$

with $E_{B_{\text {reco }}}^{*}$ the energy of the reconstructed $B_{\text {reco }}$ candidate and $E_{\text {beam }}^{*}$ the beam energy, both measured in the center-of-mass (c.m.) frame. For correctly reconstructed $B_{\text {reco }}$ candidates $\Delta E$ is expected to be close to zero within the detector resolution.

The energy-substituted mass is defined as

$$
m_{\mathrm{ES}}=\sqrt{\left(E_{\text {beam }}^{*}\right)^{2}-\left(\vec{p}_{B_{\text {reco }}^{*}}^{*}\right)^{2}}
$$

with $\vec{p}_{B_{\text {reco }}}^{*}$ the momentum of the $B_{\text {reco }}$ candidate in the c.m. frame. It is the invariant mass of the $B_{\text {reco }}$ candidate replacing its energy by the beam energy and peaks at the $B$-meson mass for correctly reconstructed candidates.

The impact of the unstable beam energy reported by PEP-II, which is only known within sizable uncertainties, on $m_{\mathrm{ES}}$ has been investigated by members of the BABAR collaboration [74]. It results in fluctuating positions of the peak of the $m_{\mathrm{ES}}$ distribution. Therefore, corrections to the beam energy have been determined for different run ranges to shift the $m_{\mathrm{ES}}$ peak to the nominal $B$-meson mass [75]. The corrections are in the order of a few $\mathrm{MeV}$. They are applied whenever $m_{\mathrm{ES}}$ distributions are build during this analysis. 


\subsubsection{Reconstruction Formalism}

The basic idea of the semi-exclusive $B$ meson reconstruction is to search for decays $B^{0} \rightarrow$ $D^{(*)-} Y^{+}$and $B^{+} \rightarrow \bar{D}^{(*) 0} Y^{+}$. The $Y$ is a placeholder for any hadronic system composed of charged and neutral pions and kaons. Intermediate resonances such as $\rho$ or $a_{1}$ mesons are not explicitly reconstructed, a fact which is represented by the name semi-exclusive reconstruction. The $D$ or $D^{*}$ meson is reconstructed at first in various decay modes and then combined with one charged track compatible with the charge of the $B$ meson to be reconstructed. This combination is called "seed". Then, additional $\pi^{0}$, that is $\gamma \gamma$ combinations with invariant mass cuts, and $K_{S}^{0}$ candidates, that is two pion combinations with invariant mass cuts, or pairs of charged tracks $\pi^{ \pm}$or $K^{ \pm}$are added. This addition is restricted by the following boundary conditions,

$$
n_{\pi}+n_{K} \leq 5, \quad n_{K_{S}^{0}} \leq 2, \quad n_{\pi^{0}} \leq 2 .
$$

This combination is then called a $D^{(*)} Y$ combination. Whenever a $D^{(*)} Y$ combination has values of $\Delta E$ and $m_{\mathrm{ES}}$ within dedicated selection windows, the $B_{\text {reco }}$ candidate is saved in the skim. These requirements are $m_{\mathrm{ES}}>5.20 \mathrm{GeV} / c^{2}$ and mode dependent cuts on $|\Delta E|$ between 30 and $90 \mathrm{MeV}$, corresponding to three standard deviations, which have been determined in previous investigations.

In total, there are 53 possible compositions of the $Y$ system, leading together with the various $D$ and $D^{*}$ reconstruction channel to more than thousand modes for the $B_{\text {reco. If }}$ a lepton with a momentum larger than $0.8 \mathrm{GeV} / c$ is required within the remaining particles, only about $400 B_{\text {reco }}$ decay modes remain. Among these, the mode most frequently reconstructed in data is $B^{+} \rightarrow \bar{D}^{0} \pi^{+} \pi^{0}$ with $\bar{D}^{0} \rightarrow K^{+} \pi^{-} \pi^{0}$.

The $m_{\mathrm{ES}}$ distributions for each reconstructed mode is fitted with the sum of a signal and a background function to determine the number of correctly reconstructed $B_{\text {reco }}$ candidates

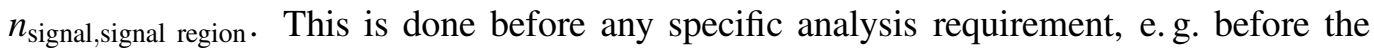
requirement of an identified lepton in this analysis. Therefore, the $m_{\mathrm{ES}}$ signal region is defined as $m_{\mathrm{ES}}>5.27 \mathrm{GeV} / c^{2}$. The purity defined as

$$
\mathcal{P}=\frac{n_{\text {signal,signal region }}}{n_{\text {all,signal region }}}
$$

where $n_{\text {all,signal region }}$ is the total number of events in the signal region, is determined and saved for each mode. Adding multiple $B_{\text {reco }}$ decay modes in the order of their individual purity (starting from the mode with the highest purity), yields the overall purity of these events, the so-called integrated purity $\mathcal{P}_{\text {int }}$, which is tabulated as well. The number $N_{\text {signal,signal region }}$ of signal events is gained from a fit to the overall $m_{\mathrm{ES}}$ distribution of the added decay modes,

$$
\mathcal{P}_{\text {int }}=\frac{N_{\text {signal,signal region }}}{N_{\text {all,signal region }}} .
$$

Of course the individual purity and the integrated purity are highly correlated by construction. However, they are not directly interchangeable, which is due to fluctuations during the fitting procedures. The variable $\mathcal{P}_{\text {int }}$ is, however, more descriptive as it refers to a whole dataset, and therefore it is used in this analysis. Restricting the analysis to decay modes yielding an integrated purity larger than $60 \%$ leaves 125 different decay modes, among which the decay mode $B^{+} \rightarrow \bar{D}^{0} \pi^{+}$with $\bar{D}^{0} \rightarrow K^{+} \pi^{-} \pi^{0}$, is the most frequent one. 


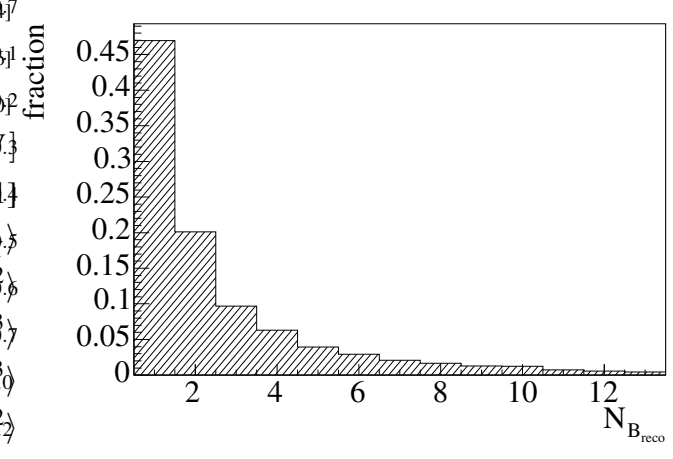

Figure 6.1: Number of $B_{\text {reco }}$ candidates, fulfilling the BSemiExcl selection criteria on $m_{\mathrm{ES}}$ and $\Delta E$, per event. About $53 \%$ of the events have more than one candidate. No identified lepton is required in the remaining particles.
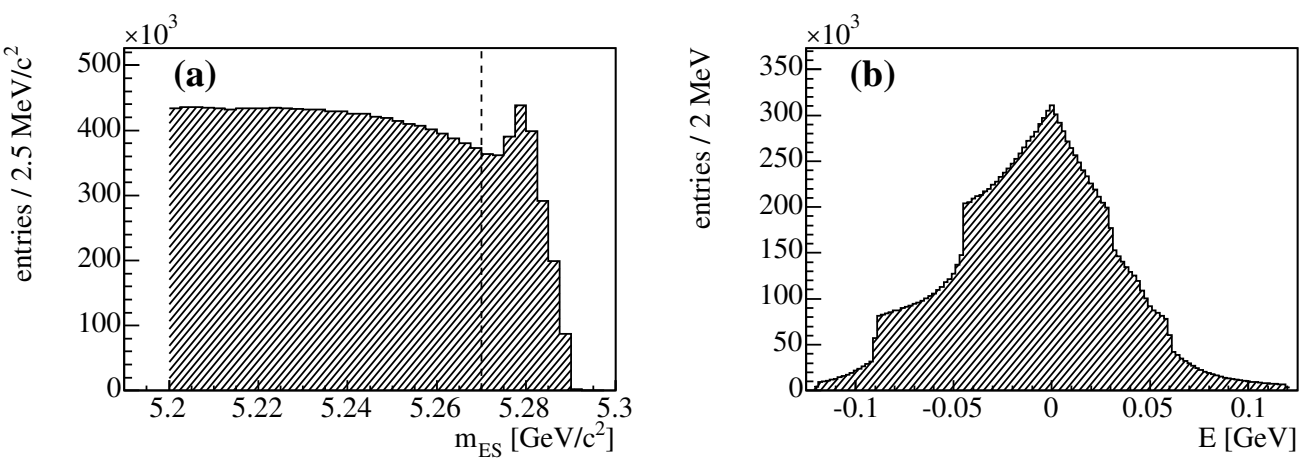

Figure 6.2: Distributions of $m_{\mathrm{ES}}$ (a) and $\Delta E$ (b) (both after the kinematic fit of the $B_{\text {reco }}$ candidate) for the best $B_{\text {reco }}$ candidate in each event (all $B_{\text {reco }}$ modes, no identified lepton required). The dashed line in (a) shows the $m_{\mathrm{ES}}$ signal region $m_{\mathrm{ES}}>5.27 \mathrm{GeV} / c^{2}$. The edges in (b) arise from the different mode dependent cuts on $\Delta E$ applied before the kinematic fit.

\subsubsection{Selection of one $B_{\text {reco }}$ Candidate per Event}

The reconstruction procedure for $B_{\text {reco }}$ candidates described above allows the construction of several candidates per event. In fact, without the requirement of an identified lepton, we find on average 1.8 candidates per event, corresponding to $47 \%$ of events with only one $B_{\text {reco }}$ (see figure 6.1). In this analysis the candidate with the highest integrated purity is chosen, minimizing the chance of picking a combinatorial candidate. This $B_{\text {reco }}$ candidate is kinematically fitted, constraining all intermediate particle masses, such as $D^{(*)}, K_{S}^{0}$, and $\pi^{0}$, to their nominal values. The $m_{\mathrm{ES}}$ and $\Delta E$ distributions of the remaining best candidates before any further requirements are shown in figure 6.2. A signal of correctly reconstructed $B_{\text {reco }}$ candidates is clearly visible in the $m_{\mathrm{ES}}$ distribution as a peak at the $B$-meson mass. However, a large fraction of combinatorial background is still selected.

\subsection{Selection of Particles Originating from the Semileptonic Decay}

All tracks and neutral candidates not associated to the selected $B_{\text {reco }}$, the so-called recoil of the $B_{\text {reco }}$, are considered to belong to the signal $B$ meson $\left(B_{\mathrm{sl}}\right)$. Among these particle 
candidates a semileptonic decay $B \rightarrow X_{c} \ell v$ is searched. Therefore, after the identification of a charged lepton, all remaining particle candidates are combined to the inclusive hadronic system. Consequently it is necessary to apply dedicated quality cuts to reject fake tracks and clusters faking neutral particles. Especially the latter are known to be not very well simulated by $\mathrm{MC}$ and thus difficult to correct for on a simulation basis. The better treatment, thus, is to identify and omit them for the further analysis. Among the selected charged tracks, an identified lepton is required to select the semileptonic decay. This is an important step in the event reconstruction described in section 6.3.3. The presented requirements have been optimized by members of the $B A B A R$ collaboration $[58,76]$ for analyses with similar conditions.

\subsubsection{Reconstruction of Charged Tracks}

As described in chapter 5.2 charged tracks are reconstructed using combined information from the SVT and the DCH. Only very low momentum tracks, as those pions originating from a $D^{*}$ decay, only leave tracking information in the SVT. As among $B \rightarrow X_{c} \ell v$ decays, $D^{*}$ decays are the most frequent ones, these SVT-only tracks are kept in the analysis, given that they have a low transverse momentum. The main purpose of the quality cuts described below is to ensure a track reconstruction with high quality and efficiency on the one hand, and to reject fake tracks on the other hand.

Acceptance Cut: $23.5^{\circ} \leq \theta \leq 145^{\circ}$, with $\theta$ the track's polar angle in the laboratory. This cut assures that the track lies within the EMC acceptance (compare to figure 5.7).

Momentum Cuts: Several cuts on the track's momentum reconstructed in the laboratory frame are applied.

- $|\vec{p}|<10 \mathrm{GeV} / c$, higher momenta cannot be achieved at PEP.

- $p_{t}>60 \mathrm{MeV} / c$, with $p_{t}$ the component of the momentum vector transverse to the beam axis. This minimal momentum is required for a reliable track measurement.

- $p_{t}<200 \mathrm{MeV} / c$. This cut is only applied to tracks, which are only measured in the SVT and have no DCH hits (so-called SVT-only tracks). As pointed out above, these tracks may arise from pions originating from $D^{*}$ decays.

- For identified electrons and muons (see section 6.3.3) additional cuts on the momentum measured in the laboratory frame are applied to ensure well defined selection efficiencies. These are $p_{\text {lab }}>0.3 \mathrm{GeV} / c$ for electrons and $p_{\text {lab }}>$ $0.6 \mathrm{GeV} / c$ for muons.

Beam Spot Region: $\left|d_{x y}\right|<1.5 \mathrm{~cm}$ and $\left|d_{z}\right|<5 \mathrm{~cm}$, where $d_{x y}$ and $d_{z}$ refer to the distance of closest approach of the track to the primary vertex in the plane perpendicular and along the beam axis, respectively. Both cuts aim at a discrimination against tracks not originating from beam-beam interactions.

Looper Rejection: A so-called looper rejection is applied against low momentum tracks that loop within the DCH and do not reach the EMC. Looping tracks with a radius of half of the DCH radius $\left(r_{\text {loop }} \approx 40 \mathrm{~cm}\right.$ ) are possible for tracks originating from the 
interaction point with transverse momenta $p_{t} \lesssim 200 \mathrm{MeV}$. The tracking algorithms, however, usually reconstruct loop tracks as several smaller segments, each of them describing one half-turn of the helix. Thus, multiple reconstructed tracks originate from one physical particle, which need to be reduced to one single track. Therefore, looper candidates are selected by applying the following criteria:

$$
\begin{aligned}
& p_{t}^{i}<250 \mathrm{MeV} / c \text { and }\left|\Delta p_{t}\right| \equiv\left|p_{t}^{i}-p_{t}^{j}\right|<120 \mathrm{MeV} / c, \\
& \left|\cos \theta^{i}\right|<0.2 \text { corresponding to } 78^{\circ}<\theta^{i}<102^{\circ}, \\
& |\Delta \phi|<5.7^{\circ} \text { and }|\Delta \theta|<5.7^{\circ} \quad \text { for same }- \text { sign tracks, } \\
& |\pi-| \Delta \phi||<5.7^{\circ} \text { and }|\pi-\Delta \theta|<5.7^{\circ} \quad \text { for opposite }- \text { sign tracks. }
\end{aligned}
$$

Finally, the candidate with the smallest impact parameter $d_{z}$ is retained while the other looper candidates are rejected.

Ghost Track Rejection: Likewise cuts to reject fake or so-called ghost tracks are applied, that is if the DCH hits of one single particle are split to build two tracks. As these two candidates basically follow the same trajectory, they can be rejected by the following requirements:

$$
\begin{aligned}
& p_{t}^{i}<350 \mathrm{MeV} / c, \\
& \left|\Delta p_{t}\right|<150 \mathrm{MeV} / c, \\
& |\Delta \phi|<5.7^{\circ}, \\
& |\Delta \theta|<5.7^{\circ}, \\
& N_{\mathrm{DCH}}^{1}<45-N_{\mathrm{DCH}}^{2} .
\end{aligned}
$$

When a pair of ghost tracks is found, the one with more DCH hits is kept while the other one is rejected.

The number of tracks cut by each of these requirements has been investigated during this analysis for all of the used datasets. These numbers are summarized in table 6.3. It is found that about $63 \%$ of the tracks are retained, this number varies only little with the investigated dataset. The cut with the largest impact is the $\left|d_{x y}\right|$ cut, rejecting roughly $21 \%$ of the tracks. The ghost and looper rejection only veto less than $0.5 \%$ of all tracks each.

\subsubsection{Requirements for Neutral Particle Candidates}

In this analysis all neutral clusters measured in the calorimeter (EMC) are assumed to be photons. No composite reconstruction such as $\pi^{0} \rightarrow \gamma \gamma$ is performed. The clusters in the EMC have to meet the following requirements to be considered as a neutral candidate:

Acceptance Cut: $18^{\circ}<\theta_{\text {clus }}<140^{\circ}$, where $\theta_{\text {clus }}$ is the polar angle of the cluster's centroid. This selection criteria ensures that the whole cluster is entirely located inside the EMC.

Minimal Number of Crystals: $N_{\text {crys }}>2$, with $N_{\text {crys }}$ the number of crystals in the cluster. Clusters with only one or two crystals are most likely background. 
Table 6.3: Summary of rejection rates for the charged track selection criteria. The percentage is calculated relative to the original number of tracks before any cut. This study is performed on a subset of the respective data samples.

\begin{tabular}{lrrrrrr}
\hline \multirow{2}{*}{ Criteria } & \multicolumn{5}{c}{ Rejected tracks } \\
& Gen. MC & {$[\%]$} & Cockt. MC & {$[\%]$} & Data & {$[\%]$} \\
\hline Total number & 666814 & 100.0 & 818988 & 100.0 & 159116 & 100.0 \\
\hline Acceptance $\left(23.5^{\circ} \leq \theta \leq 145^{\circ}\right)$ & 34668 & 5.2 & 43004 & 5.3 & 7452 & 4.7 \\
$|\vec{p}|<10 \mathrm{GeV} / c$ & 551 & 0.1 & 691 & 0.1 & 139 & 0.1 \\
$p_{t}>60 \mathrm{MeV} / c$ & 21975 & 3.3 & 34495 & 4.2 & 3762 & 2.4 \\
$p_{t}<200 \mathrm{MeV} / c($ SVT-only) & 18758 & 2.8 & 23581 & 2.9 & 5565 & 3.5 \\
$\left|d_{x y}\right|<1.5 \mathrm{~cm}$ & 144846 & 21.7 & 152883 & 18.7 & 33158 & 20.8 \\
$\left|d_{z}\right|<5 \mathrm{~cm}$ & 26364 & 4.0 & 28580 & 3.5 & 6441 & 4.0 \\
Looper & 2544 & 0.4 & 2116 & 0.3 & 654 & 0.4 \\
Ghosts & 1710 & 0.3 & 1569 & 0.2 & 424 & 0.3 \\
\hline Rejected number & 251416 & 37.7 & 286919 & 35.0 & 57595 & 36.2 \\
\hline
\end{tabular}

Minimal Energy: $E_{\text {clus }}>50 \mathrm{MeV}$, where $E_{\text {clus }}$ is the deposited energy in the EMC cluster. Again this cut fights against background, e.g. originating from beam background.

Shower Shape: The shape of a shower in the EMC can be used to distinguish energy depositions from photons or electrons from those from hadrons. Photons and electrons tend to deposit most of their energy in the central crystals, while the latter distribute their energy more uniformly. This shape can be quantized by the lateral moment of a shower (LAT) [77]. The variable LAT varies between 0 and 1 and is calculated using each crystals energy $E_{\text {crys }, i}$ and the radius of each crystal to the center of the cluster $r_{i}$,

$$
\text { LAT }=\frac{\sum_{i=3}^{N_{\text {crys }}} E_{\text {crys }, i} r_{i}^{2}}{E_{\text {crys }, 1} r_{0}^{2}+E_{\text {crys }, 2} r_{0}^{2}+\sum_{i=3}^{N_{\text {crys }}} E_{\text {crys }, i} r_{i}^{2}} \quad \text { with } r_{0} \simeq 5 \mathrm{~cm} .
$$

A cut LAT $<0.6$ is applied to select clusters originating from electromagnetic showers rather than those from hadronic ones.

Split-Off Rejection: Another cut is applied to reject so-called split-off clusters, originating from interactions of a charged hadron with the EMC but separated from the track's point of impact on the EMC and thus faking the presence of an additional particle. Therefore the angle difference of the cluster and any track's point of impact on the EMC surface is calculated,

$$
\Delta \alpha \equiv \arccos \left[\cos \theta_{\text {clus }} \cos \theta_{\text {trk }}+\sin \theta_{\text {clus }} \sin \theta_{\text {trk }} \cos \left(\phi_{\text {clus }}-\phi_{\text {trk }}\right)\right]
$$

For each photon, this angle difference $\Delta \alpha$ is required to be larger than $0.08\left(4.6^{\circ}\right)$ for all tracks not identified as electrons. 
Table 6.4: Summary of rejection rates for the neutral candidate selection criteria. The percentage is calculated relative to the original number of neutrals before any cut. This study is performed on a subset of the respective data samples.

\begin{tabular}{lrrrrrr}
\hline \multirow{2}{*}{ Criteria } & \multicolumn{7}{c}{ Rejected photons } \\
\hline Total number & 1232754 & 100.0 & 1416005 & 100.0 & 230418 & 100.0 \\
\hline $18^{\circ}<\theta_{\text {clus }}<140^{\circ}$ & 48057 & 3.9 & 64949 & 4.6 & 9878 & 4.3 \\
$N_{\text {crys }}>2$ & 124901 & 10.1 & 158218 & 11.2 & 17902 & 7.8 \\
$E_{\text {clus }}>50 \mathrm{MeV}$ & 267378 & 21.7 & 348032 & 24.6 & 42869 & 18.6 \\
LAT $<0.6$ & 33745 & 2.7 & 41314 & 2.9 & 7624 & 3.3 \\
$\Delta \alpha>4.6^{\circ}$ & 17596 & 1.4 & 19396 & 1.4 & 4752 & 2.1 \\
\hline Rejected number & 491677 & 39.9 & 631909 & 44.6 & 83025 & 36.0 \\
\hline
\end{tabular}

As for the tracks, the number of neutral candidates rejected by each of these requirements has been investigated during this analysis. These numbers are summarized in table 6.4. We find that about $20 \%$ of the clusters are rejected by the lower cut on the cluster energy. In total, $55 \%, 60 \%$, and $64 \%$ of the neutrals are retained in Cocktail MC, generic MC, and data, respectively. The variation of these fractions indicates that energy depositions from neutral particles and the number of neutral particles with a certain energy, e.g. originating from beam background, is modeled worse in MC than it is the case for charged particles. Further comparisons between data and MC are shown later and the treatment of neutral particles is studied in detail as systematic effect.

\subsubsection{Particle Identification}

The most important purpose of the BABAR particle identification algorithms in this analysis is the identification of the charged lepton for the selection of the semileptonic decay. Furthermore, within the inclusive $X_{c}$ system, tracks have to be identified as a specific particle type for assigning the right particle energy.

The performance of a PID algorithm for a particle type $a$ is usually characterized by its efficiency, that is the fraction of true particles of type $a$ it identifies, and the misidentification rate for other particles types $t_{i}$, that is the fraction of true particles of type $t_{i}$ which are identified to be of type $a$.

We use the particle selectors described in the following. Performance plots for all selectors are available from ref. [65].

1. Leptons from the semileptonic decay of the $B_{\mathrm{sl}}$ meson are identified as follows:

a) Electrons are identified using a likelihood based selector named PidLHElectrons [78]. This identification algorithm combines information from the EMC, the DCH, and the DIRC. Specifically, these are the energy loss $\mathrm{d} E / \mathrm{d} x$ in the $\mathrm{DCH}$, the shower shape in the EMC, e.g. as measured by the variable LAT, the energy-momentum ratio measured in the DCH and EMC, $E_{\mathrm{EMC}} /\left|\vec{p}_{\mathrm{DCH}}\right|$, and the Cherenkov angle $\theta_{C}$ measured in the DIRC. This electron selector is 
characterized by a selection efficiency larger than $90 \%$, while the misidentification rates for pions, kaons, and protons are below $2 \%$, $5 \%$, and $2 \%$, respectively. However, these numbers are only valid for momenta above $0.8 \mathrm{GeV} / c$, measured in the laboratory frame, and a minimal momentum in the laboratory frame of $0.3 \mathrm{GeV} / c$ is required for reasonable results of the PID algorithm. As we measure the hadronic moments with a lepton-momentum cutoff at $0.8 \mathrm{GeV} / c$ in the $B$ meson rest-frame, the high quality of the selector is achieved in any case.

b) Muons are identified by the neural net based selector muNNTight [79], based on information provided by the IFR combined with the deposited energy in the EMC cluster. The performance of this selector is significantly worse than it is for the electrons, but still works reliably enough to include muons in this analysis. It achieves an efficiency between $70 \%$ and $80 \%$ for laboratory momenta above $1.5 \mathrm{GeV} / c$. For lower momenta, below $1.5 \mathrm{GeV} / c$, the efficiency drops rapidly below $50 \%$. To avoid the worst momentum region, only muons with a momentum in the laboratory frame above $0.6 \mathrm{GeV} / c$ are selected in this analysis. Another difference w.r.t. the electron selector is the increased fake rate for pions. About $2 \%$ to $4 \%$ of all pions are identified as muons which will turn out as a significant background for this analysis. For kaons a fake rate of $0.5 \%$ to $1 \%$ is achieved.

2. Within the remaining particles, kaons and protons are identified and the respective particle mass is assigned to determine the track's energy. All tracks not identified as kaons or protons are assigned the pion mass.

a) Charged kaons are identified using a likelihood based algorithm called KLHTight [80] with overall selection efficiencies between $80 \%$ and $90 \%$ for momenta between $0.5 \mathrm{GeV} / c$ and $3 \mathrm{GeV} / c$. The pion misidentification rate is below $2 \%$ in this momentum region and only rises at momenta above $3.5 \mathrm{GeV} / c$.

b) Protons are identified with the selector pLHTight with a selection efficiency between $80 \%$ and $100 \%$ depending on the momentum range. The fake rates for pions and kaons are in the region of $1 \%$ to $3 \%$. This selector is by far the least relevant for this analysis.

Particle identification is performed on data and on simulated events. However, the efficiencies for each particle type, which includes the misidentification rates, might differ in these two datasets. This has been studied on MC and data control samples by members of the BABAR collaboration and correction methods for MC have become standard within the computing framework [81]. This so-called PID tweaking method is only applied to $\mathrm{MC}$ events and it adds or removes tracks to or from each list of identified particles with the probability of the efficiency difference between data and MC. This correction method is applied in the used MC samples. 


\subsection{Event Selection Criteria}

Within all selected charged tracks in the recoil of the fully reconstructed $B_{\text {reco, }}$, an identified lepton, electron or muon (cf. section 6.3.3), is searched. Events with none or more than one charged lepton are rejected from the event sample. The hadronic system $X_{c}$ is reconstructed as sum of all four-vectors of the remaining tracks $\left(N_{\text {trk }, X}\right)$ and neutrals $\left(N_{\text {neutral }, X}\right)$, excluding the lepton,

$$
P_{X_{c}}=\sum_{i=1}^{N_{\text {trk }, X}} P_{i, \text { trk }}+\sum_{i=1}^{N_{\text {neutral }, X}} P_{i, \text { neutral }} .
$$

A kinematic consistency check is provided by the well known initial state and the measured four-vector of the $B_{\text {reco. }}$. From these quantities, the missing four-vector can be calculated,

$$
P_{\text {miss }}=P_{\Upsilon(4 S)}-P_{B_{\text {reco }}}-P_{\ell}-P_{X_{c}},
$$

using also the measured lepton four-momentum and the combined $X_{c}$ four-momentum. This four-vector, in the case of a semileptonic decay $B \rightarrow X_{c} \ell v$ and a correctly reconstructed lepton and $X_{c}$ system, will fulfill the requirement

$$
P_{\text {miss }}^{2}=m_{\text {miss }}^{2}=E_{\text {miss }}^{2}-\left(c\left|\vec{p}_{\text {miss }}\right|\right)^{2}=0
$$

equivalent to

$$
E_{\text {miss }}-c\left|\vec{p}_{\text {miss }}\right|=0, E_{\text {miss }}>0 \text {, and }\left|\vec{p}_{\text {miss }}\right|>0
$$

within the detector resolution. The variable $E_{\text {miss }}-c\left|\vec{p}_{\text {miss }}\right|$ will be used for three purposes. On the one hand, background can be rejected with cuts on this variable, as here no accumulation around zero is expected. On the other hand, also badly reconstructed signal events $B \rightarrow X_{c} \ell v$ might not have a value of $E_{\text {miss }}-c\left|\vec{p}_{\text {miss }}\right|$ near zero, if for example a kaon or pion of the $X_{c}$ decay escapes the detector acceptance. As for a precise determination of the hadronic moments a good reconstruction of the hadronic system is necessary, the variable $E_{\text {miss }}-c\left|\vec{p}_{\text {miss }}\right|$ helps to select those events. In fact, this variable has an advantage over the more intuitive variable $m_{\text {miss }}$, cf. [82]. As shown in figure 6.3, the resolution of $E_{\text {miss }}-c\left|\vec{p}_{\text {miss }}\right|$ is in contrast to the one of missing mass squared independent of the neutrino momentum. Due to a correlation between the neutrino momentum and the mass or energy of the hadronic system, which is in the order of $20 \%$, the resolution of $m_{\text {miss }}^{2}$ also differs for different final states. If one wants to apply selection criteria defined by a certain resolution, it would be necessary to rescale any cut on $m_{\text {miss }}^{2}$ with the missing momentum. In contrast, we can keep one common cut on $E_{\text {miss }}-c\left|\vec{p}_{\text {miss }}\right|$, namely $-0.2<E_{\text {miss }}-c\left|\vec{p}_{\text {miss }}\right|$ $<0.3 \mathrm{GeV}$. A third reason to cut on $E_{\text {miss }}-c\left|\vec{p}_{\text {miss }}\right|$ is that it helps to reject regions, in which the MC simulation describes the data insufficiently, this will be shown in section 6.9.1.

Other requirements on the whole event are imposed to subtract background and assure a good reconstruction of the signal decays $B \rightarrow X_{c} \ell v$ :

1. To assure a well defined lepton identification and to reject secondary leptons not originating directly from a $B$-meson decay, the analysis is performed with a minimal lepton momentum in the $B$ meson rest frame, $p_{\ell, \mathrm{BRF}} \geq 0.8 \mathrm{GeV} / c$. This cut, of course, varies, when moments with different cuts on $p_{\ell, \mathrm{BRF}}$ are measured. 

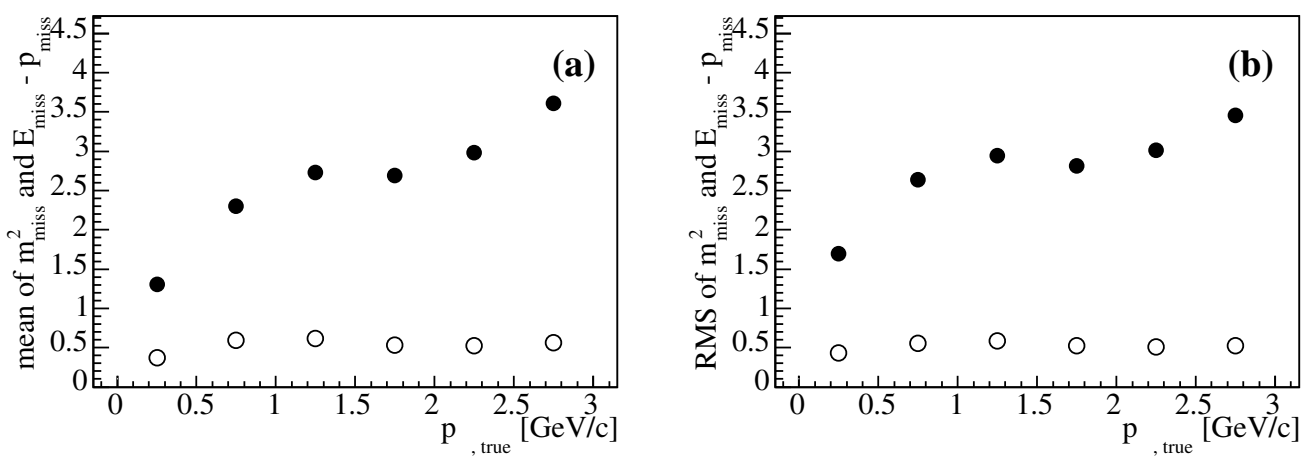

Figure 6.3: Dependence of $m_{\text {miss }}^{2}(\bullet)$ and $E_{\text {miss }}-c\left|\vec{p}_{\text {miss }}\right|$ (०) resolution on $p_{v \text {,true }}$ (a) shows the mean of the corresponding distribution, (b) its RMS. It is clearly visible that the variable $E_{\text {miss }}-c\left|\vec{p}_{\text {miss }}\right|$ is independent of the missing momentum, while $m_{\text {miss }}^{2}$ shows a dependence. These plots are produced for $B \rightarrow X_{c} \ell v$ decays in the $m_{\mathrm{ES}}$ signal region on Cocktail MC.

2. From the more than $400 B_{\text {reco }}$ decay modes we select only those, leading to an integrated purity $\mathcal{P}_{\text {int }}>60 \%$. As mentioned above, this cut leaves about 125 decay modes.

3. The properly reconstructed $B_{\text {reco }}$ candidates are selected by a cut $m_{\mathrm{ES}}>5.27 \mathrm{GeV} / c^{2}$.

4. To improve the reconstruction quality, we omit reconstructed $X_{c}$ systems only containing neutral particles, $N_{\text {trk }, X} \geq 1$.

5. Leptons from secondary decays such as semileptonic decays of $D$ mesons can be rejected by a requirement on the correct charge correlation between the reconstructed lepton on the $b$ quark inside the $B_{\text {reco }}: q_{b_{\text {reco }}} \cdot q_{\ell}<0$. Only if a neutral $B$ meson oscillates before its decay, this cut cannot fulfill this purpose.

6. Another cut aiming at the accumulation of well reconstructed signal decays, rejects events with a large electrical charge imbalance: $\left|Q_{\text {tot,event }}\right|=\left|q_{B_{\text {reco }}}+q_{\ell}+q_{X_{c}}\right| \leq 1$. The total charge of all tracks in the event is required to be less or equal one. But if the $X_{c}$ system consists of only one charged track (and additional neutral clusters) $Q_{\text {totevent }}$ has to be zero. This cut has been investigated as discussed in section 6.9.2.

7. We require a positive missing momentum and energy in the event $E_{\text {miss }}>0.0 \mathrm{GeV}$ and $\left|\vec{p}_{\text {miss }}\right|>0.0 \mathrm{GeV} / c$.

8. $-0.2<E_{\text {miss }}-c\left|\vec{p}_{\text {miss }}\right|<0.3 \mathrm{GeV}$. This serves, as mentioned above, three different purposes. It not only rejects background or badly reconstructed signal events, but also helps to reject regions, in which the MC simulation describes the data insufficiently. This cut has been investigated in several ways and will be discussed in section 6.9.3.

The amount of events rejected by each of these requirements have been investigated during this analysis. The numbers are summarized in table 6.5 . 
Table 6.5: Number of events remaining after each cut in signal decays, background decays, and in data. Please note that these numbers still contain combinatorial background, in case of data also residual background.

\begin{tabular}{lllllll}
\hline & signal & \multicolumn{3}{c}{ background } & \multicolumn{2}{c}{ data } \\
& 2005131 & \multicolumn{2}{c}{4485459} & \multicolumn{2}{c}{1970874} & \\
\hline cut & ev. remaining & \% cut & ev. remaining & \% cut ev. remaining & \% cut \\
\hline$p_{\ell, \text { BRF }} \geq 0.8 \mathrm{GeV} / c$ & 1759808 & 12.2 & 990065 & 77.9 & 890375 & 54.8 \\
$\mathcal{P}_{\text {int }}$ & 339456 & 80.7 & 136893 & 86.2 & 114212 & 87.2 \\
$m_{\text {ES }}$ & 234261 & 31.0 & 78118 & 42.9 & 61524 & 46.1 \\
$\ell / B_{\text {reco }}$ charge corr. & 226522 & 3.3 & 65551 & 16.1 & 47467 & 22.9 \\
$Q_{\text {tot }} \leq 1$ & 217318 & 4.1 & 63693 & 2.8 & 35609 & 25.0 \\
$E_{\text {miss }}$ & 217310 & 0.0 & 63567 & 0.2 & 35167 & 1.2 \\
$\left|\vec{p}_{\text {miss }}\right|$ & 217310 & 0.0 & 63567 & 0.0 & 35167 & 0.0 \\
$E_{\text {miss }}-c\left|\vec{p}_{\text {miss }}\right|$ & $62465(3.12 \%)$ & 71.3 & $9625(0.21 \%)$ & 84.9 & $13452(0.68 \%)$ & 61.8 \\
\hline
\end{tabular}

The cuts and their impact on resolution and statistical uncertainty have been investigated during this analysis. Although some of the tests could already been shown at this stage of the documentation, some rely on the complete extraction formalism of the hadronic moments. Therefore, these studies are discussed after the complete description of this method in section 6.9.

\subsection{Reconstruction of the Hadronic System}

As discussed before, the first step to reconstruct the inclusive $X_{c}$ system is to add all measured four-momenta of the remaining tracks and neutrals in the event to build the $X_{c}$ fourvector (see eq. (6.8)). Given the well known initial state, which is a great advantage of lepton colliders w.r.t. hadron colliders, the resolution can be improved by exploiting kinematic constraints. The four-vectors of all particles are varied within given uncertainties to fulfill these constraints using the minimization method of least squares. As the uncertainty of the inclusive $X_{c}$ system is dominated by unmeasured particles rather than misreconstruction of existing tracks or neutrals, the error-matrix of this four-momentum is derived from MC simulations. For the lepton and $B_{\text {reco }}$ momentum, the usual BABAR track-fit error-matrices are used.

While the mathematical formalism can be found in textbooks [83], the implementation written in $\mathrm{C}++$ and embedded in the BABAR computing framework has been a task during this thesis, performed together with J.E. Sundermann, who worked on a similar topic. This computing package is documented in [84]. 


\subsubsection{Method of Least Squares with Constraints}

A given problem may contain $n$ measured parameters $\vec{y}, p$ unmeasured parameters $\vec{a}$ and $m$ constraints $\vec{f}$. These requirements $\vec{f}$ will be fulfilled for the true parameters $\bar{a}$ and $\bar{y}$.

$$
\begin{aligned}
f_{1}\left(\bar{a}_{1}, \bar{a}_{2}, \ldots, \bar{a}_{p}, \bar{y}_{1}, \bar{y}_{2}, \ldots, \bar{y}_{n}\right) & =0 \\
f_{2}\left(\bar{a}_{1}, \bar{a}_{2}, \ldots, \bar{a}_{p}, \bar{y}_{1}, \bar{y}_{2}, \ldots, \bar{y}_{n}\right) & =0 \\
\vdots & \\
f_{m}\left(\bar{a}_{1}, \bar{a}_{2}, \ldots, \bar{a}_{p}, \bar{y}_{1}, \bar{y}_{2}, \ldots, \bar{y}_{n}\right) & =0 .
\end{aligned}
$$

In general, due to uncertainties in the experiment, the measured values $\vec{y}$ will not solve the constraints. Corrections $\Delta \vec{y}$ can be calculated, so that the sum $\vec{y}^{\prime}=\vec{y}+\Delta \vec{y}$ fulfills the equations (6.12). From these, also the unmeasured parameters can be calculated. The corrections $\Delta \vec{y}$ are gained from the minimization of the squared sum

$$
S(\vec{y})=\Delta \vec{y}^{T} \mathcal{V}^{-1} \Delta \vec{y},
$$

with $\mathcal{V}$ the covariance matrix of the measured parameters.

A general method to determine local extrema of non-linear functions of many variables is the definition of Lagrange Multipliers. It introduces $m$ additional so-called Lagrange Multipliers $\lambda_{k}$, one for each constraint of the problem. A solution of the problem can then be found by finding the extrema of the function

$$
L(\vec{y}, \vec{a}, \vec{\lambda})=S(\vec{y})+2 \sum_{k=1}^{m} \lambda_{k} f_{k}(\vec{y}, \vec{a})
$$

with respect to all parameters $\vec{y}, \vec{a}$, and $\vec{\lambda}$. The necessary condition for a local minimum of this function is then equivalent to the condition for a minimum of $S(\vec{y})$ under the constraint $f_{k}(\vec{y}, \vec{a})=0$.

With linear constraints the solution can be found in one step, otherwise the problem has to be solved iteratively, linearizing it in every iteration.

\subsubsection{Linearization of Non-linear Problems}

The linearized constraints as defined in eq. (6.12) are given as follows,

$$
\begin{aligned}
f_{k}\left(\vec{y}^{\prime}, \vec{a}^{\prime}\right) \approx f\left(\vec{y}^{*}, \vec{a}^{*}\right) & +\sum_{j=1}^{p} \frac{\partial f_{k}}{\partial a_{j}} \cdot\left(\Delta a_{j}-\Delta a_{j}^{*}\right) \\
& +\sum_{i=1}^{n} \frac{\partial f_{k}}{\partial y_{i}} \cdot\left(\Delta y_{i}-\Delta y_{i}^{*}\right) \approx 0,
\end{aligned}
$$

where $\vec{y}(\vec{a})$ contains the start values of the measured (unmeasured) parameters, $\vec{y}^{*}\left(\vec{a}^{*}\right)$ contains the values of the measured (unmeasured) parameters after the previous iteration, and $\vec{y}^{\prime}\left(\vec{a}^{\prime}\right)$ contains the values of the measured (unmeasured) parameters of the next iteration. All functional values and derivatives are calculated at $\vec{y}^{*}=\vec{y}+\Delta \vec{y}^{*}$ and $\vec{a}^{*}=\vec{a}+\Delta \vec{a}^{*}$. The difference of the start values and the next iteration is given by $\vec{y}^{\prime}=\vec{y}+\Delta \vec{y}$ and $\vec{a}^{\prime}=\vec{a}+\Delta \vec{a}$. 
These equations can be written in vector/matrix notation,

$$
\vec{f}^{*}+\mathcal{A}\left(\Delta \vec{a}-\Delta \vec{a}^{*}\right)+\mathcal{B}\left(\Delta \vec{y}-\Delta \vec{y}^{*}\right) \approx 0
$$

or

$$
\mathcal{A} \Delta \vec{a}+\mathcal{B} \Delta \vec{y}-\vec{c}=0 \quad \text { with } \quad \vec{c}=\mathcal{A} \Delta \vec{a}^{*}+\mathcal{B} \Delta \vec{y}^{*}-\vec{f}^{*} .
$$

The vector $\vec{c}$ in eq. (6.17) is for iteration $n$ a constant vector which only depends on quantities of the previous iteration $(n-1)$. In equations (6.16) and (6.17) the matrices $\mathcal{A}$ and $\mathcal{B}$ are defined as follows:

$$
\mathcal{A}=\frac{\partial \vec{f}}{\partial \vec{a}} \quad \text { and } \quad \mathcal{B}=\frac{\partial \vec{f}}{\partial \vec{y}}
$$

In this notation the function $L$ to be minimized is

$$
L=\Delta \vec{y}^{T} \mathcal{V}^{-1} \Delta \vec{y}+2 \vec{\lambda}^{T}(\mathcal{A} \Delta \vec{a}+\mathcal{B} \Delta \vec{y}-\vec{c}) .
$$

After differentiating w.r.t. $\vec{y}, \vec{a}$ and $\vec{\lambda}$ the following conditions for an extremum are obtained,

$$
\begin{aligned}
\mathcal{V}^{-1} \Delta \vec{y}+\mathcal{B}^{T} \vec{\lambda} & =0 \\
\mathcal{A}^{T} \vec{\lambda} & =0 \\
\mathcal{B} \Delta \vec{y}+\mathcal{A} \Delta \vec{a} & =c .
\end{aligned}
$$

These $(n+p+m)$ equations will be solved for the unknown values $\Delta \vec{y}, \Delta \vec{a}$ and $\vec{\lambda}$.

\subsubsection{Solution of the Linearized Problem}

Writing the system of coupled differential equations defined in eq. (6.20) in only one equation with partitioned matrices, yields

$$
\left(\begin{array}{ccc}
\mathcal{V}^{-1} & 0 & \mathcal{B}^{T} \\
0 & 0 & \mathcal{A}^{T} \\
\mathcal{B} & \mathcal{A} & 0
\end{array}\right)\left(\begin{array}{c}
\Delta \vec{y} \\
\Delta \vec{a} \\
\lambda
\end{array}\right)=\left(\begin{array}{l}
0 \\
0 \\
c
\end{array}\right) .
$$

To find a solution of eq. (6.21) the abbreviations

$$
\mathcal{V}_{\mathcal{B}}=\left(\mathcal{B} \mathcal{V B}^{T}\right)^{-1} \quad \text { and } \quad \mathcal{V}_{\mathcal{A}}=\left(\mathcal{A}^{T} \mathcal{V}_{\mathcal{B}} \mathcal{A}\right)
$$

are introduced. The inverse of the matrix in eq. (6.21) has to be calculated. Therefore, its special form with $\mathcal{B}(\mathcal{A})$ and $\mathcal{B}^{T}\left(\mathcal{A}^{T}\right)$ as partition matrices can be exploited. Firstly, the inverse can also be written in a partitioned form and conditions for its partition matrices can be formulated,

$$
\left(\begin{array}{ccc}
\mathcal{V}^{-1} & 0 & \mathcal{B}^{T} \\
0 & 0 & \mathcal{A}^{T} \\
\mathcal{B} & \mathcal{A} & 0
\end{array}\right)^{-1}=\left(\begin{array}{ccc}
\mathcal{C}_{11} & \mathcal{C}_{21}^{T} & \mathcal{C}_{31}^{T} \\
\mathcal{C}_{21} & \mathcal{C}_{22}^{T} & \mathcal{C}_{32}^{T} \\
\mathcal{C}_{31} & \mathcal{C}_{32} & \mathcal{C}_{33}
\end{array}\right)
$$


with the conditions

$$
\begin{aligned}
\mathcal{C}_{11}= & \mathcal{V}-\mathcal{V B}^{T} \mathcal{V}_{\mathcal{B}} \mathcal{B V} \\
& +\mathcal{V B}^{T} \mathcal{V}_{\mathcal{B}} \mathcal{A} \mathcal{V}_{\mathcal{A}}{ }^{-1} \mathcal{A}^{T} \mathcal{V}_{\mathcal{B}} \mathcal{B} \mathcal{} \\
\mathcal{C}_{21}= & -\mathcal{V}_{\mathcal{A}}{ }^{-1} \mathcal{A}^{T} \mathcal{V}_{\mathcal{B}} \mathcal{B} \mathcal{V} \\
\mathcal{C}_{22}= & \mathcal{V}_{\mathcal{A}}{ }^{-1} \\
\mathcal{C}_{31}= & \mathcal{V}_{\mathcal{B}} \mathcal{B} \mathcal{V}-\mathcal{V}_{\mathcal{B}} \mathcal{A} \mathcal{V}_{\mathcal{A}}{ }^{-1} \mathcal{A}^{T} \mathcal{V}_{\mathcal{B}} \mathcal{B} \mathcal{V} \\
\mathcal{C}_{32}= & \mathcal{V}_{\mathcal{B}} \mathcal{A} \mathcal{V}_{\mathcal{A}}{ }^{-1} \\
\mathcal{C}_{33}= & -\mathcal{V}_{\mathcal{B}}+\mathcal{V}_{\mathcal{B}} \mathcal{A} \mathcal{V}_{\mathcal{A}}{ }^{-1} \mathcal{A}^{T} \mathcal{V}_{\mathcal{B}}
\end{aligned}
$$

The corrections $\Delta \vec{y}$ and $\Delta \vec{a}$ as well as the Lagrange Multipliers $\vec{\lambda}$ are calculated by multiplication with $\vec{c}$,

$$
\begin{aligned}
\Delta \vec{y} & =\mathcal{C}_{31}^{T} \vec{c}=\left(\mathcal{V B}^{T} \mathcal{V}_{\mathcal{B}}-\mathcal{V} \mathcal{B}^{T} \mathcal{V}_{\mathcal{B}} \mathcal{A} \mathcal{V}_{\mathcal{A}}{ }^{-1} \mathcal{A}^{T} \mathcal{V}_{\mathcal{B}}\right) \vec{c} \\
\Delta \vec{a} & =\mathcal{C}_{32}^{T} \vec{c}=\left(\mathcal{V}_{\mathcal{A}}{ }^{-1} \mathcal{A}^{T} \mathcal{V}_{\mathcal{B}}\right) \vec{c} \\
\vec{\lambda} & =\mathcal{C}_{33} \vec{c}=\left(-\mathcal{V}_{\mathcal{B}}+\mathcal{V}_{\mathcal{B}} \mathcal{A}_{\mathcal{A}}{ }^{-1} \mathcal{A}^{T} \mathcal{V}_{\mathcal{B}}\right) \vec{c}
\end{aligned}
$$

The iteration is repeated until certain convergence criteria are met assuring that the squared sum $S$ has reached a minimum and all constraints are fulfilled. Therefore, we define convergence criteria,

$$
\frac{S(n-1)-S(n)}{\mathrm{ndf}}<\varepsilon_{S} \quad \text { and } \quad \sum_{k=1}^{m} f_{k}^{(n)}(\vec{y}, \vec{a})<\varepsilon_{F},
$$

where $n$ denotes the number of iterations and ndf defines the number of degrees of freedom which is given by difference of the number of constraints and the number of unmeasured parameters, ndf $=m-p$. The parameter $\varepsilon_{S}$ defines the size of the allowed change of $S$ from one iteration to the next. The precision by which the constraints have to be fulfilled is defined by the parameter $\varepsilon_{F}$.

\subsubsection{Use in This Analysis}

The above-described method can be applied to the decay reconstructed in this analysis, $\Upsilon(4 S) \rightarrow B_{\text {reco }} B_{\mathrm{sl}}$ with $B_{\mathrm{sl}} \rightarrow X_{c} \ell \nu$. The 11 measured parameters in this case are,

- four measured components of the $B_{\text {reco }}$ four-momentum,

- four measured components of the $X_{c}$ four-momentum,

- and three independent components of the lepton momentum $\vec{p}_{\ell}$. The mass of the lepton is constrained to the muon or electron mass, respectively.

In addition, there are three unmeasured parameters,

- three components of the neutrino momentum $\vec{p}_{v}$, constraining its mass to zero.

Furthermore the following kinematic constraints can be required: 
- Energy conservation:

$$
f_{1}=E_{B_{\text {reco }}}+E_{X_{c}}+E_{\ell}+E_{v}-E_{\Upsilon(4 S)}=0
$$

- Momentum conservation:

$$
f_{2,3,4}=\vec{p}_{B_{\text {reco }}}+\vec{p}_{X_{c}}+\vec{p}_{\ell}+\vec{p}_{V}-\vec{p}_{\Upsilon(4 S)}=0
$$

- Equal $B$-meson masses:

$$
\begin{aligned}
f_{5} & =m_{B_{\text {reco }}}^{2}-m_{B_{\mathrm{sl}}}^{2}=\left[E_{B_{\text {reco }}}^{2}-\vec{p}_{B_{\text {reco }}}^{2}\right]-\left[\left(E_{X_{c}}+E_{\ell}+E_{V}\right)^{2}-\left(\vec{p}_{X_{c}}+\vec{p}_{\ell}+\vec{p}_{v}\right)^{2}\right] \\
& =0
\end{aligned}
$$

Consequently, the missing mass of the event is constrained to zero. The last constraint of equal $B$-meson masses is less stringent than constraining both masses to the nominal value. But it avoids convergence problems, which might arise for momentum and energy constellations giving a mass slightly deviating from the nominal value.

The covariance matrices needed for the fit are derived in different ways for the respective particles. For the lepton, the track-fit covariance matrix supplied by the BABAR dataset is used. Similarly, the $B_{\text {reco }}$ candidate brings a covariance matrix resulting from the primary kinematic fit done during its reconstruction. Only the combined $X_{c}$ system suffers from particles emerging the detector acceptance. Thus, any error-matrix taken from reconstruction would underestimate its uncertainty. Therefore, the uncertainties are studied on MC simulations and suitable covariance matrices are build. In fact, we find that the resolution differs for different regions in the detector and with the number of particles used for building the $X_{c}$ system. Therefore, the resolution of any component $a_{\text {meas }}-a_{\text {true }}$ is studied in five bins of the multiplicity and five bins in $\theta$,

- multiplicity $\in[1,3],[4,6],[7,9],[10,11],[12, \infty[$ and

$$
\text { - } \theta \in\left[0 ., 30.0^{\circ}\right],\left[30.0^{\circ}, 60.0^{\circ}\right],\left[60.0^{\circ}, 90.0^{\circ}\right],\left[90.0^{\circ}, 108.9^{\circ}\right],\left[108.9^{\circ}, 180.0^{\circ}\right] \text {. }
$$

We use Cartesian coordinates $E, p_{x}, p_{y}, p_{z}$ for the parameterization of the four-vector. While for the $x$ and $y$ component of the momentum vector, the resolution is in the order of $0.2 \mathrm{GeV} / c$ and follows very well a Gaussian shape, the $z$ component shows a resolution of $0.25 \mathrm{GeV} / c$ to $0.45 \mathrm{GeV} / c$. The distribution $p_{z \text {,meas }}-p_{z \text {,true }}$ furthermore shows a nonGaussian tail to negative values in events with low multiplicity. Nevertheless, we use the Gaussian fit as an adequate approximation. A similar behavior is valid for the resolution of the energy, as shown in figure 6.4. Resolutions between $0.3 \mathrm{GeV}$ and $0.5 \mathrm{GeV}$ are observed. The extracted resolutions for all four components, as measured by the $\sigma$ of the Gaussian fit, are shown in figure 6.5 .

Several different parameterizations of the four-vector have been investigated, such as spherical coordinates or a special one, in which the momentum coordinates are rescaled w. r. t. the measured momentum $\vec{p}$. None of them showed an improvement in the shape of the resolutions. Thus, Cartesian coordinates have been chosen for the fit as they show the largest improvement in terms of the resolution and bias of the variable $n_{X}^{2}$. 

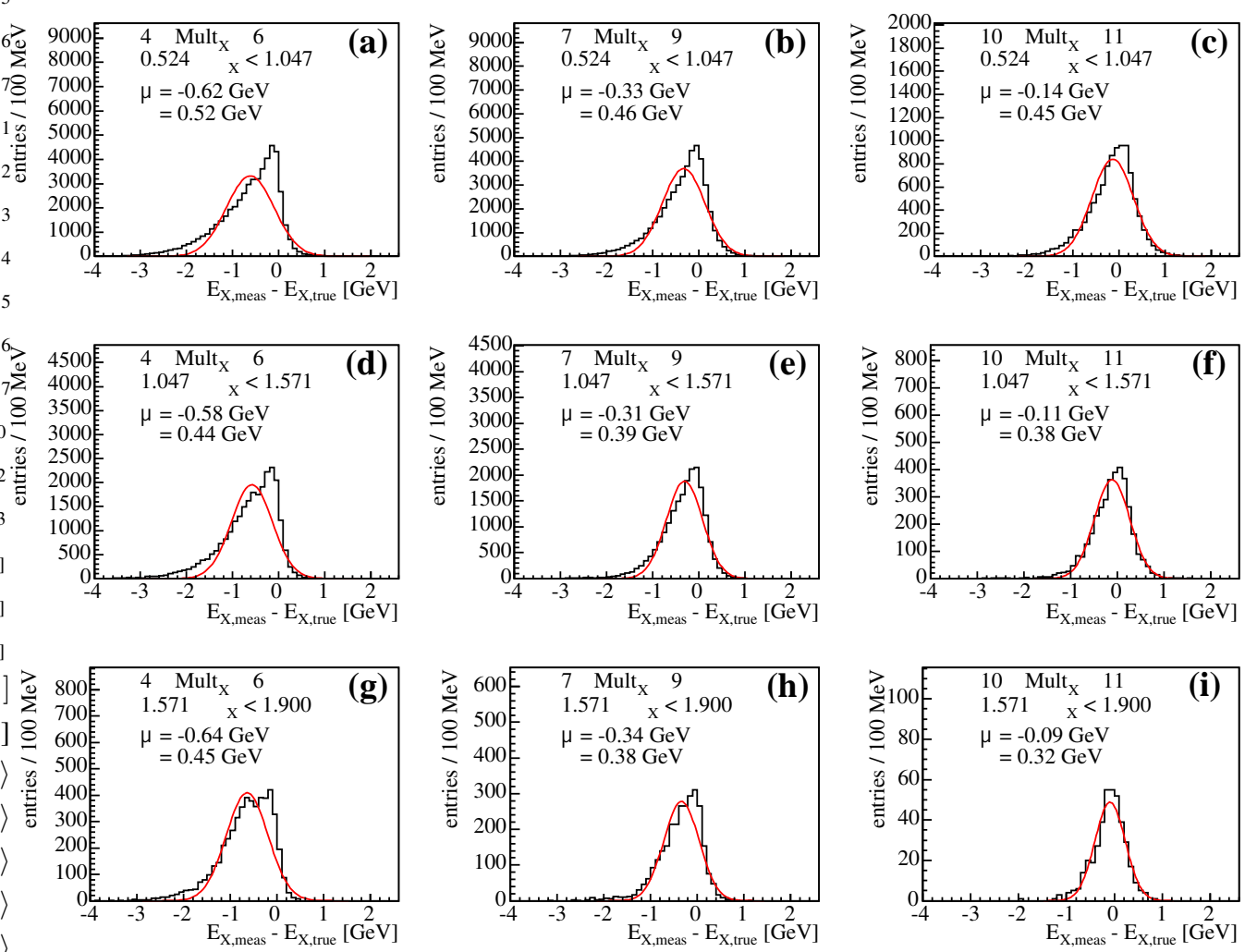

Figure 6.4: Energy resolution of the $X_{c}$ system used for the kinematic fit. The different histograms (a)-(i) show the resolution in different exemplary bins (the ones with the largest number of events) of the $X_{c}$ multiplicity and azimuthal angle $\theta$. The red line shows a fit with a Gaussian used to estimate the resolution. 

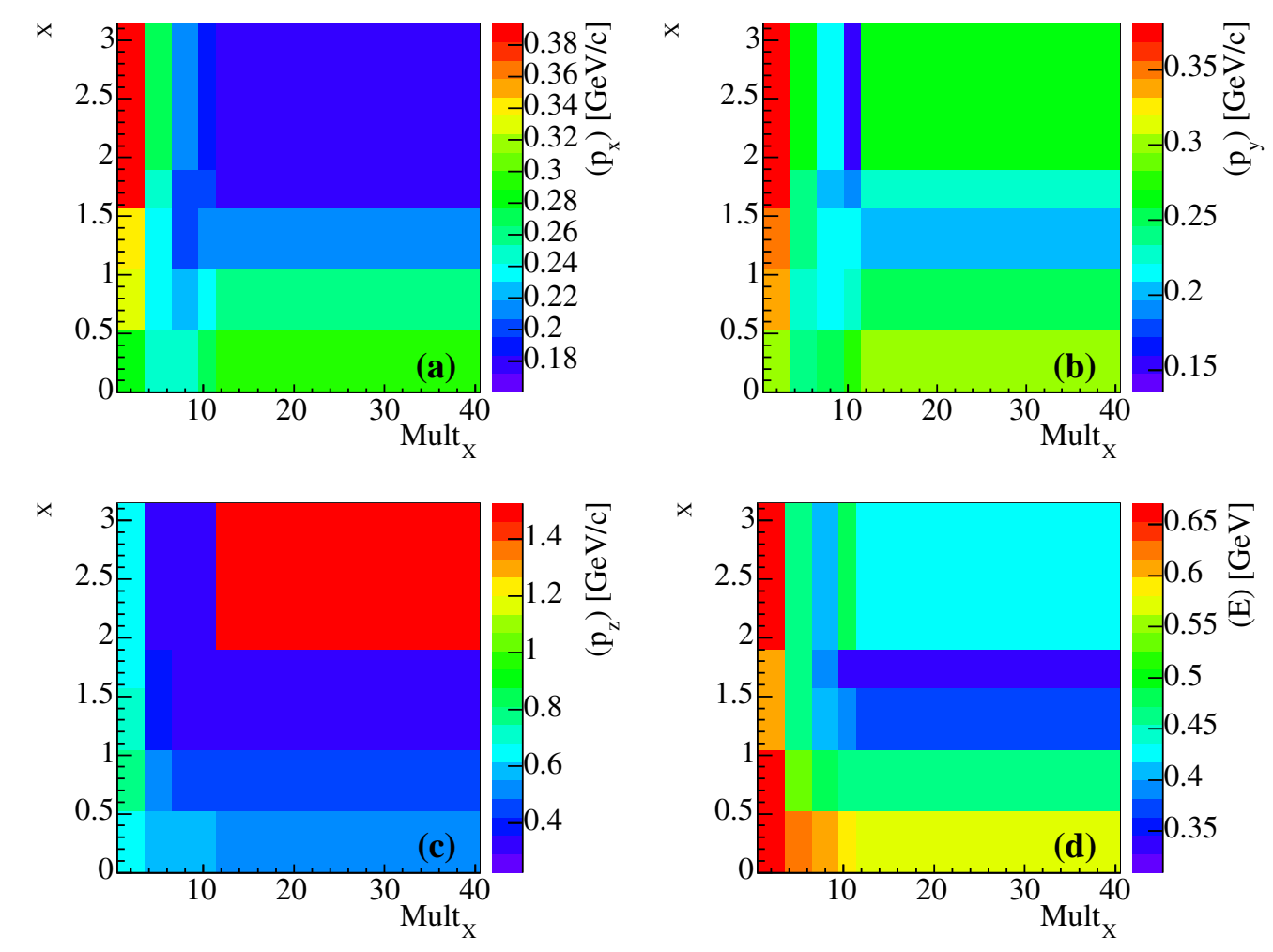

Figure 6.5: Resolution of all four components of the $X_{c}$ four-vector in Cartesian coordinates ((a) $p_{x}$, (b) $p_{y}$, (c) $p_{z}$, and (d) $E$ ). The resolutions are estimated by Gaussian fits in bins of azimuthal angle $\theta_{X}$ and multiplicity Mult $X_{X}$ of the $X_{c}$ system.

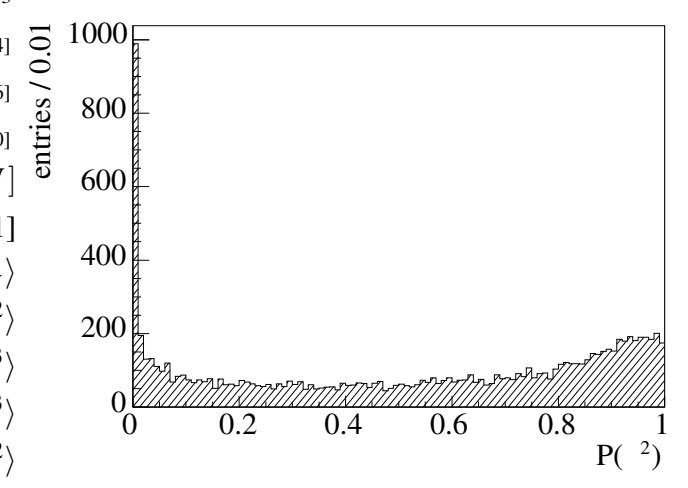

Figure 6.6: Probability of $\chi^{2}$ for the final fit performed on data. $10 \%$ of the events accumulate at $\mathcal{P}\left(\chi^{2}\right)=0$. The shape is well modelled by $\mathrm{MC}$ simulations. 

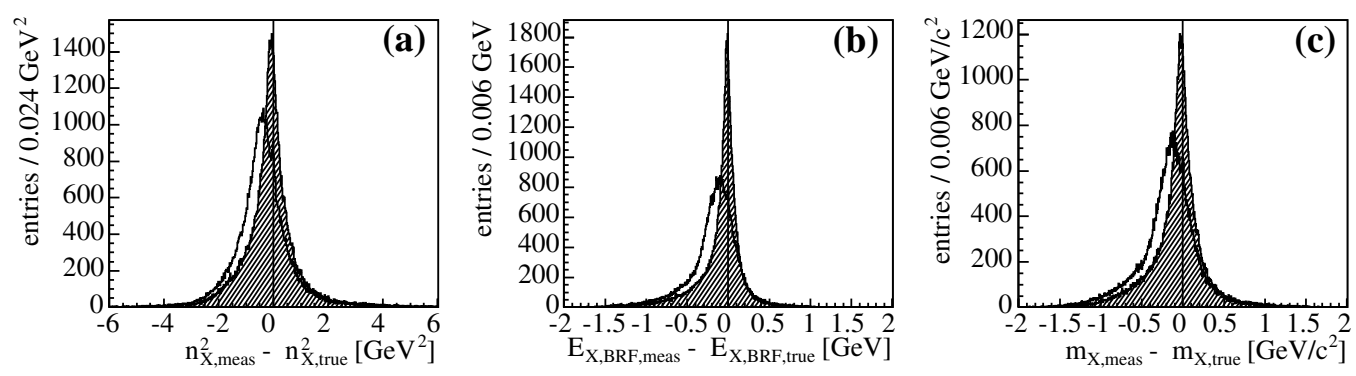

Figure 6.7: Resolution and bias before (white histogram) and after (hatched histogram) the kinematic fit of $n_{X}^{2}$ (a), $E_{X, B R F}(\mathrm{~b})$, and $m_{X}$ (c). All event selection criteria are applied. The bias is reduced for all three variables remarkably. The numbers are given in table 6.6 .

\begin{tabular}{llrr}
\hline value & & before & after \\
\hline$n_{X}^{2}\left[\mathrm{GeV}^{2}\right]$ & bias & -0.37 & -0.11 \\
& RMS & 1.17 & 1.05 \\
\hline$E_{X, \mathrm{BRF}}[\mathrm{GeV}]$ & bias & -0.18 & -0.09 \\
& RMS & 0.32 & 0.28 \\
\hline$m_{X}\left[\mathrm{GeV} / c^{2}\right]$ & bias & -0.17 & -0.07 \\
& RMS & 0.37 & 0.33 \\
\hline
\end{tabular}

Table 6.6: Mean and RMS of the distributions shown in figure 6.7 for $n_{X}^{2}, E_{X, \mathrm{BRF}}$, and $m_{X}$. The numbers are given before and after the kinematic fit.

The reliability of the fit in terms of pull distributions has been tested extensively. The final $\chi^{2}$ probability distribution, however, is not totally flat, but still reasonable given the non-Gaussian shapes of the covariances of the $X_{c}$ system (see figure 6.6). Only $10 \%$ of the events accumulate at $\mathcal{P}\left(\chi^{2}\right)=0$, however, they do not worsen the resolution of $n_{X}^{2}$. As the shape is well modelled by MC simulations, all fitted events are kept for the analysis.

To demonstrate the gain of the kinematic fit, the resolution of the variable of interest, $n_{X}^{2}$, before and after the kinematic fit is plotted in figure 6.7, the numbers are summarized in table 6.6. As $n_{X}^{2}$ is a difference of the invariant mass and the energy of the $X_{c}$ system, these variables are investigated, too. It is found that in all three variables the bias, that is the mean of the distribution $n_{\mathrm{X} \text {,meas }}^{2}-n_{\mathrm{X} \text {,true }}^{2}$ (analogously for $E_{X, \mathrm{BRF}}$ and $m_{X}$ ), is reduced remarkably by the kinematic fit. For $n_{X}^{2}$, it is reduced from $-0.37 \mathrm{GeV}^{2}$ to $-0.11 \mathrm{GeV}^{2}$. The RMS of the respective distributions, however, decreases only slightly.

\subsection{The $n_{X}^{2}$ Distribution}

The variable $n_{X}^{2}$ is a combination of the invariant mass and the energy in the $B$ meson rest frame, as defined in eq. (1.1). This difference of two measured quantities makes the shape of its distribution less familiar than it is for example for the invariant mass alone, which has distinct peaks at the $D$ or $D^{*}$ mass, respectively. Therefore, the $n_{X}^{2}$ distribution for signal decays as simulated by the $B A B A R$ event generator is shown in figure 6.8. It ranges from 0 to $12 \mathrm{GeV}^{2}$ for a lower cut on the lepton momentum $p_{\ell, \mathrm{BRF}}$ at $0.8 \mathrm{GeV} / c$. The range decreases to a maximum value of $5.5 \mathrm{GeV}^{2}$ for a tighter cut on $p_{\ell, \mathrm{BRF}} \geq 1.7 \mathrm{GeV} / c$. The 

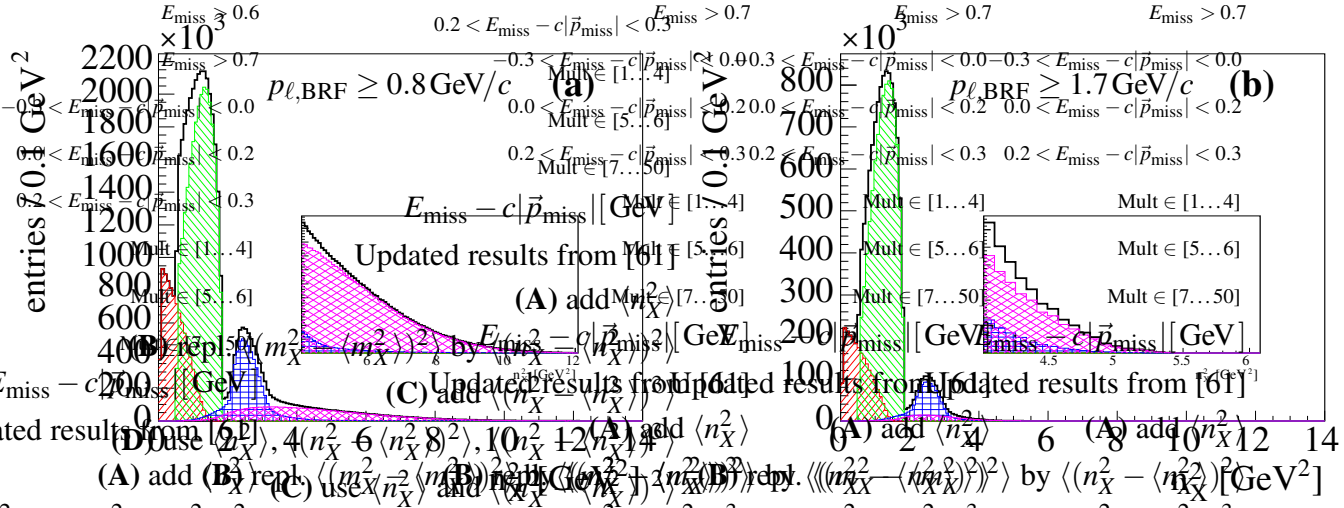

Figure 6.8: $n_{X}^{2}$ distribution on generator level for $p_{\ell, \mathrm{BRF}} \geq 0.8 \mathrm{GeV} / c$ (a) and $p_{\ell, \mathrm{BRF}} \geq$ $1.7 \mathrm{GeV} / c$ (b). The components are $B \rightarrow D \ell v$ (远), $B \rightarrow D^{*} \ell v(\mathbb{E}), B \rightarrow D^{* *} \ell v$ (曲), and $B \rightarrow D^{(*)} \pi \ell v$ (圆). The insets show the endpoint of the spectrum.

distributions for the different exclusive channels such as $B \rightarrow D \ell v$ or $B \rightarrow D^{*} \ell v$ are not totally separated as in the mass spectrum, but the $B \rightarrow D \ell v$ decays populate the smallest values of $n_{X}^{2}$ while the excited states and the non resonant decays contribute to the high energy tail of the distribution.

In the reconstructed $n_{X}^{2}$ spectrum for signal decays in the generic $B \bar{B} \mathrm{MC}$, the distribution is smeared out and also negative values of $n_{X}^{2}$ are measured, while the upper bound is comparable to the one in the true distributions.

\subsection{Background Subtraction}

Although the event selection criteria already suppress a large amount of background, still about $20 \%$ of all selected events are not of the type $B \rightarrow X_{c} \ell v$. Therefore, a background subtraction method is implemented, which effectively weights away the background contribution in the calculation of the moments. This method is discussed in this section.

Two sources of background are distinguished:

1. Combinatorial background originating from misreconstructed $B_{\text {reco }}$ candidates and from events $e^{+} e^{-} \rightarrow q \bar{q}$ with $q=u, d, s$. Although these selected candidates have values of $m_{\mathrm{ES}}$ in the signal region, similar event topologies can be found in a signal free region in $m_{\mathrm{ES}}$, the so-called sideband. Thus, the shape and normalization of this background is measured directly on data using the $m_{\mathrm{ES}}$ sideband.

2. Residual background on the signal side are events other than decays $B \rightarrow X_{c} \ell v$. This background peaks in the $m_{\mathrm{ES}}$ signal region and is subtracted taking shape and normalization from MC simulations. All major decay channels contributing to this type of background are discussed.

A verification procedure of the subtraction method, using a sample of pure background events, is discussed at the end of this section. 


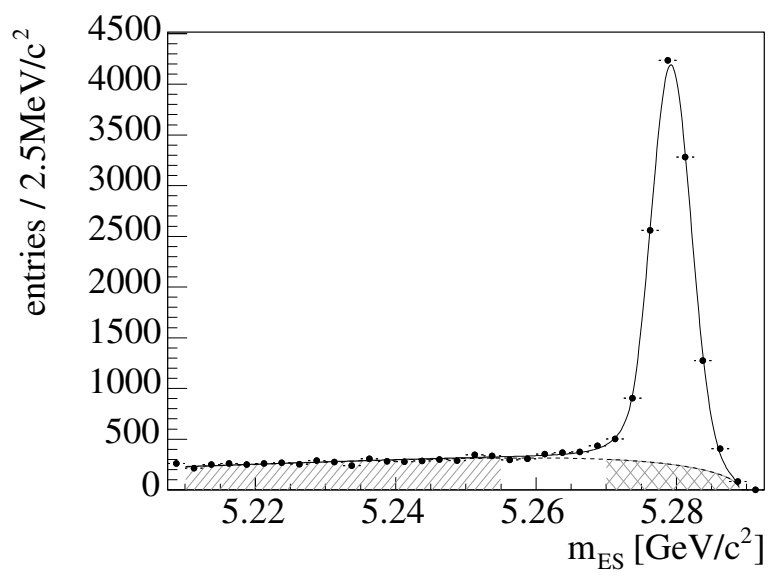

Figure 6.9: $m_{\mathrm{ES}}$ spectrum of $B_{\text {reco }}$ decays after the final event selection. The signal (solid line) and background (dashed line) components of the fit are overlaid. The crossed area shows the background under the $B_{\text {reco }}$ signal. The background control region in the $m_{\mathrm{ES}}$ sideband is indicated by the hatched area.

\subsubsection{Combinatorial $B_{\text {reco }}$ Background}

During the reconstruction of the $B_{\text {reco }}$ (cf. section 6.2) not only true hadronic $B$-meson decays are found, but also random combinations are formed stemming either from two different $B$ decays or from so-called continuum events $e^{+} e^{-} \rightarrow q \bar{q}$ with $q=u, d, s$. Calculating $m_{\mathrm{ES}}$ for these events does not yield a peak at the $B$-meson mass, but a rather flat distribution in the region from $5.2 \mathrm{GeV} / c^{2}$ to the endpoint at $5.289 \mathrm{GeV} / c^{2}$.

The amount of combinatorial background under the signal peak can be determined by fitting the $m_{\mathrm{ES}}$ distribution with the sum of a signal and a background function. The ARGUS function $f_{\mathrm{ARGUS}}[85]$ describes the shape of the combinatorial background. It is defined as

$$
\frac{\mathrm{d} N}{\mathrm{~d} m_{\mathrm{ES}}} \propto m_{\mathrm{ES}} \sqrt{1-x^{2}} e^{-\chi\left(1-x^{2}\right)},
$$

where $x=m_{\mathrm{ES}} / m_{\mathrm{ES}, \text { max }}, m_{\mathrm{ES}, \max }=5.289 \mathrm{GeV} / c^{2}$ is the kinematic endpoint approximated by half of the mean c.m. energy, and $\chi$ is a free parameter defining the curvature of the function. The signal is parameterized with a modified Gaussian function [86] peaked at the $B$-meson mass and corrected for radiation losses,

$$
\frac{\mathrm{d} N}{\mathrm{~d} m_{\mathrm{ES}}} \propto \sigma \cdot \begin{cases}e^{-\frac{\left(m_{\mathrm{ES}}-m_{0}\right)^{2}}{2 \sigma^{2}}} & \text { if } m_{\mathrm{ES}}>m_{0}-\alpha \sigma, \\ \left(1-\frac{\alpha^{2}}{n}-\frac{\alpha}{n} \frac{\left(m_{\mathrm{ES}}-m_{0}\right)}{\sigma}\right)^{-n} \cdot e^{-\frac{\alpha^{2}}{2}} & \text { if } m_{\mathrm{ES}} \leq m_{0}-\alpha \sigma,\end{cases}
$$

with the free parameters $m_{0}, \sigma, \alpha$ and $n$ and an additional normalization. The power law tail is needed to model energy losses in the EMC in the reconstruction of $\pi^{0}$ mesons. We define a signal and a sideband region in $m_{\mathrm{ES}}$ by the following bounds:

$$
\begin{aligned}
& 5.270 \mathrm{GeV} / c^{2} \leq m_{\mathrm{ES}, \text { signal }} \leq 5.289 \mathrm{GeV} / c^{2}, \\
& 5.210 \mathrm{GeV} / c^{2} \leq m_{\mathrm{ES}, \text { sideband }} \leq 5.255 \mathrm{GeV} / c^{2} .
\end{aligned}
$$

The measured $m_{\mathrm{ES}}$ spectrum in data for the selected events is shown in figure 6.9 together with the fitted sum of functions. 
The relative size $s$ of the combinatorial background in the signal region w.r.t. the sideband region is determined by the ratio of the integrals of the ARGUS function in these regions,

$$
s=\frac{\int_{\text {signal }} f_{\mathrm{ARGUS}}\left(m_{\mathrm{ES}}\right) \mathrm{d} m_{\mathrm{ES}}}{\int_{\text {sideband }} f_{\mathrm{ARGUS}}\left(m_{\mathrm{ES}}\right) \mathrm{d} m_{\mathrm{ES}}} .
$$

This scale factor $s$ is used to scale any combinatorial background distribution (e. g. in $n_{X}^{2}$ ) measured in the $m_{\mathrm{ES}}$ sideband to the respective amount in the signal region. Investigations show that the curvature of the ARGUS function in the signal region varies in different regions of the measured $n_{X}^{2}$, yielding varying scaling factors $s=s\left(n_{X}^{2}\right)$. To take this into account, the $m_{\mathrm{ES}}$ fits are performed in different bins of $n_{X}^{2}$ and the combinatorial background spectrum in the signal region is calculated as

$$
\frac{\mathrm{d} N_{\text {comb.bg }}}{\mathrm{d} n_{X}^{2}}\left(n_{X}^{2}\right)=\frac{\mathrm{d} N_{\text {sideband }}}{\mathrm{d} n_{X}^{2}}\left(n_{X}^{2}\right) \cdot s\left(n_{X}^{2}\right) .
$$

The dependence of the scaling factor on $n_{X}^{2}$ is shown in figure 6.10. At higher cuts on the lepton momentum, the number of bins in $n_{X}^{2}$ used for fitting the $m_{\mathrm{ES}}$ distribution is decreased due to the smaller number of events. We find significant variations for the lower cuts on the lepton momentum, e.g. between $s=0.21 \pm 0.04$ and $s=0.56 \pm 0.08$. The distributions of the extracted combinatorial background are as well depicted in figure 6.10.

\subsubsection{Residual Background on the Signal Side}

There are other sources of background which cannot be subtracted by the $m_{\mathrm{ES}}$ sideband method, as the $B_{\text {reco }}$ is reconstructed correctly. These are decays of the signal $B$ meson other than semileptonic decays $B \rightarrow X_{c} \ell v$.

The amount and the shape of this background peaking in $m_{\mathrm{ES}}$ is extracted in $\mathrm{MC}$ simulations. Therefore, the combinatorial background in these background events is subtracted as described in the previous section 6.7.1. The background spectrum is normalized to the number of correctly reconstructed $B_{\text {reco }}$ in data and in the total generic MC dataset, $N_{B_{\text {reco }} \text {,data }}=N_{B_{\text {reco }}, \mathrm{MC}}$.

Several sources of background are identified:

- So-called upper-vertex semileptonic decays of $D$ or $D_{s}$ mesons, which produce a lepton with the correct charge/flavor relation w.r. t. the $B_{\text {reco }}: B \rightarrow X D(\rightarrow Y \ell v)$ or $B \rightarrow X D_{s}(\rightarrow Y \ell v)$.

- Leptonic or semileptonic $B$-meson decays to $\tau$ leptons, including $\tau$ leptons from intermediate $D_{s}$ mesons, decaying leptonically into electrons or muons: $B \rightarrow \tau \rightarrow$ $e / \mu$ or $B \rightarrow D_{s}^{+} \rightarrow \tau \rightarrow e / \mu$.

- $B^{0} \bar{B}^{0}$ oscillations: If either the signal $B$ or the $B_{\text {reco }}$ oscillates before it decays, the charge/flavor correlation with the lepton is inverted. A $D$ meson produced at the socalled lower-vertex, can produce a right-charged lepton via its semileptonic decay: $B \rightarrow D(\rightarrow Y \ell v) X$. 

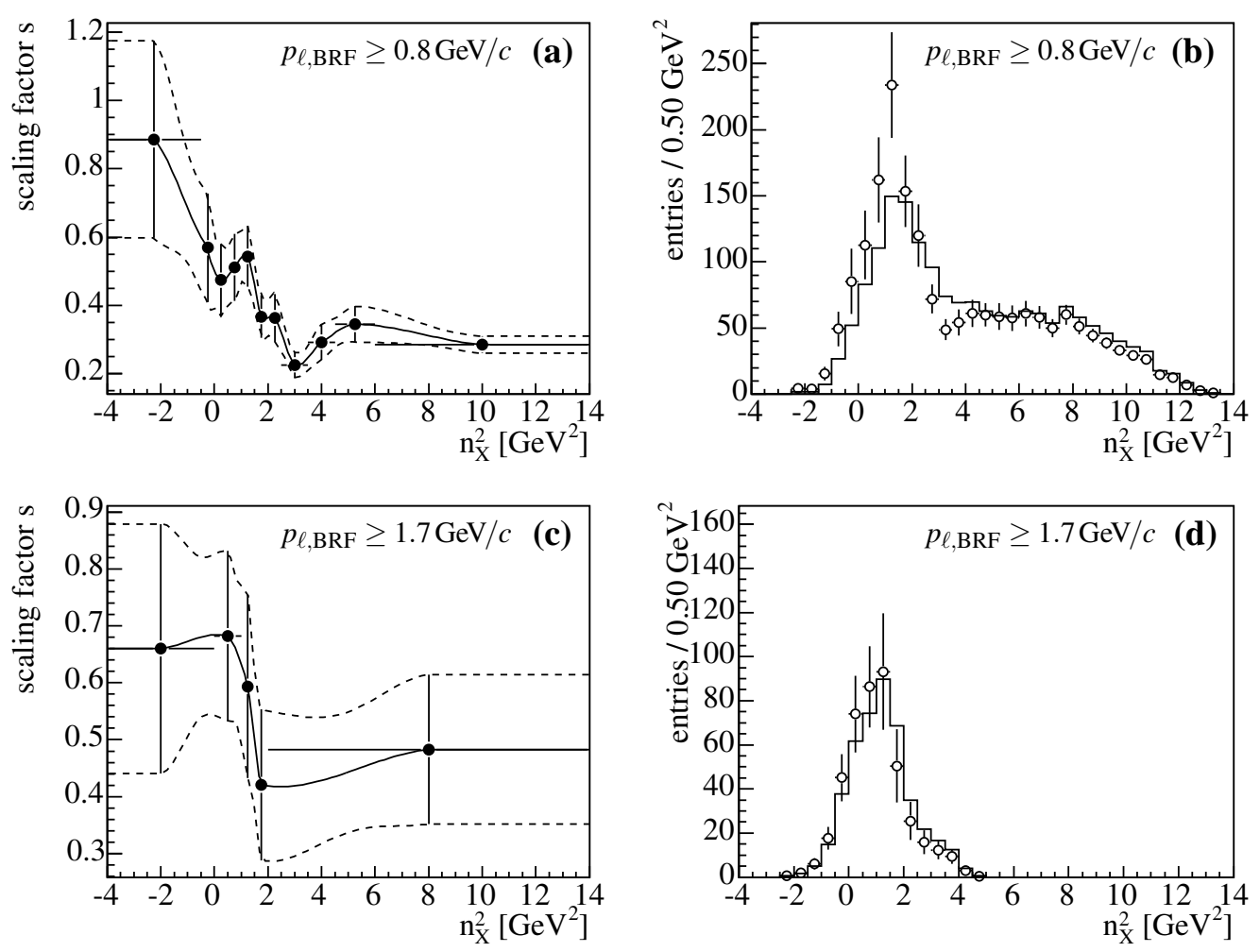

Figure 6.10: Scaling factors $s$ (a,c) extracted from $m_{\mathrm{ES}}$ fits in bins of $n_{X}^{2}$ for two different cuts $p_{\ell, \mathrm{BRF}} \geq 0.8 \mathrm{GeV} / c$ and $p_{\ell, \mathrm{BRF}} \geq 1.7 \mathrm{GeV} / c$. The error bars in $x$ indicate the bin width. To get the scaling factors at each value of $n_{X}^{2}$, they are interpolated using a cubic spline. The extracted distributions of combinatorial background are shown in $(b, d)(\circ)$. The histogram (black line) shows the corresponding distribution scaled with only one single scaling factor. 
Table 6.7: Summary of scaling factors $s$ used to correct branching fractions of background processes in $\mathrm{MC} \mathcal{B}_{\mathrm{MC}}$ to measured branching fractions $\mathcal{B}_{\text {data }}$ as discussed in section 6.7.2.

\begin{tabular}{lrrr}
\hline Decay & $\mathcal{B}_{\mathrm{MC}}[\%]$ & $\mathcal{B}_{\text {data }}[\%]$ & $s$ \\
\hline$B^{+} \rightarrow X D^{+} \rightarrow Y \ell^{+} v$ & 0.56 & $0.40 \pm 0.08[87,88]$ & $0.719 \pm 0.145$ \\
$B^{0} \rightarrow X D^{+} \rightarrow Y \ell^{+} v$ & 0.53 & $0.37 \pm 0.18[87,88]$ & $0.706 \pm 0.338$ \\
$B^{+} \rightarrow X D^{0} \rightarrow Y \ell^{+} v$ & 0.51 & $0.56 \pm 0.05[87,88]$ & $1.086 \pm 0.095$ \\
$B^{0} \rightarrow X D^{0} \rightarrow Y \ell^{+} v$ & 0.60 & $0.52 \pm 0.10[87,88]$ & $0.871 \pm 0.164$ \\
$B^{+} \rightarrow X \bar{D}^{0}, D^{-} \rightarrow Y \ell^{-} v$ & 6.60 & $7.60 \pm 0.47[24,42,88]$ & $1.151 \pm 0.071$ \\
$B^{0} \rightarrow X \bar{D}^{0}, D^{-} \rightarrow Y \ell^{-} v$ & 8.90 & $7.13 \pm 0.52[24,42,88]$ & $0.801_{-0.055}^{+0.058}$ \\
\hline$B^{+} \rightarrow X D_{s}^{+} \rightarrow Y \ell^{+} v$ & 0.81 & $0.62_{-0.10}^{+0.12}[42,87,89]$ & $0.765_{-0.123}^{+0.148}$ \\
$B^{0} \rightarrow X D_{s}^{+} \rightarrow Y \ell^{+} v$ & 0.77 & $0.81_{-0.14}^{+0.17}[42,87,89]$ & $1.052_{-0.182}^{+0.221}$ \\
\hline$B^{+} \rightarrow \tau^{+} \rightarrow \ell^{+}$ & 0.52 & $0.47 \pm 0.05[42,90,91]$ & $0.905 \pm 0.098$ \\
$B^{0} \rightarrow \tau^{+} \rightarrow \ell^{+}$ & 0.52 & $0.43 \pm 0.05[42,90,91]$ & $0.834 \pm 0.092$ \\
$B^{+} \rightarrow D_{s}^{+} \rightarrow \tau^{+} \rightarrow \ell^{+}$ & 0.17 & $0.09_{-0.02}^{+0.03}[42,87]$ & $0.524_{-0.146}^{+0.158}$ \\
$B^{0} \rightarrow D_{s}^{+} \rightarrow \tau^{+} \rightarrow \ell^{+}$ & 0.16 & $0.12_{-0.03}^{+0.04}[42,87]$ & $0.721_{-0.211}^{+0.224}$ \\
\hline$B \rightarrow J / \psi \rightarrow \ell^{+} \ell^{-}$ & $6.06 \times 10^{-2}$ & $(6.46 \pm 0.20) \times 10^{-2}[42]$ & $1.066 \pm 0.034$ \\
$B \rightarrow \psi(2 S) \rightarrow \ell^{+} \ell^{-}$ & $0.23 \times 10^{-2}$ & $(0.226 \pm 0.017) \times 10^{-2}[42]$ & $0.982 \pm 0.088$ \\
\hline$B \rightarrow X_{u} \ell v$ & 0.210 & $0.222 \pm 0.033[92]$ & $1.057 \pm 0.157$ \\
\hline
\end{tabular}

- $B$-meson decays to $J / \psi$ or $\psi(2 S)$ decaying subsequently to $\ell^{+} \ell^{-}$.

- Pions, kaons, and protons, which are misidentified as muons or electrons. This background is by far more relevant for muons than for electrons. In total, this is one of the largest sources of residual background.

- Semileptonic decays $B \rightarrow X_{u} \ell v$. Although $\left|V_{u b}\right|^{2}$ is two orders of magnitude smaller than $\left|V_{c b}\right|^{2}$, this background becomes a significant component especially for higher cuts on the lepton momentum.

- So-called mistagged $B_{\text {reco }}$ candidates peaking in $m_{\mathrm{ES}}$. These are candidates which are nearly correctly reconstructed but a slow charged or neutral pion is exchanged between the $X_{c}$ system and the $B_{\text {reco }}$ candidate. This is only a small component of the total residual background.

All relevant components are discussed in the following. To match the branching fractions in our MC simulation to the latest measurements, scaling factors are applied as weight per event, which are summarized in table 6.7.

Figure 6.11 shows a breakdown of the contributing background decay channels. Investigations show a strong difference in the background composition for different subsets of the data, e. g. for electrons and muons or neutral and charged $B$ mesons. This is illustrated in figure 6.12. Some background components, such as misidentified hadrons or background originating from $B^{0} \bar{B}^{0}$ oscillations, only contribute to the muon or neutral $B$-meson sample, respectively. 

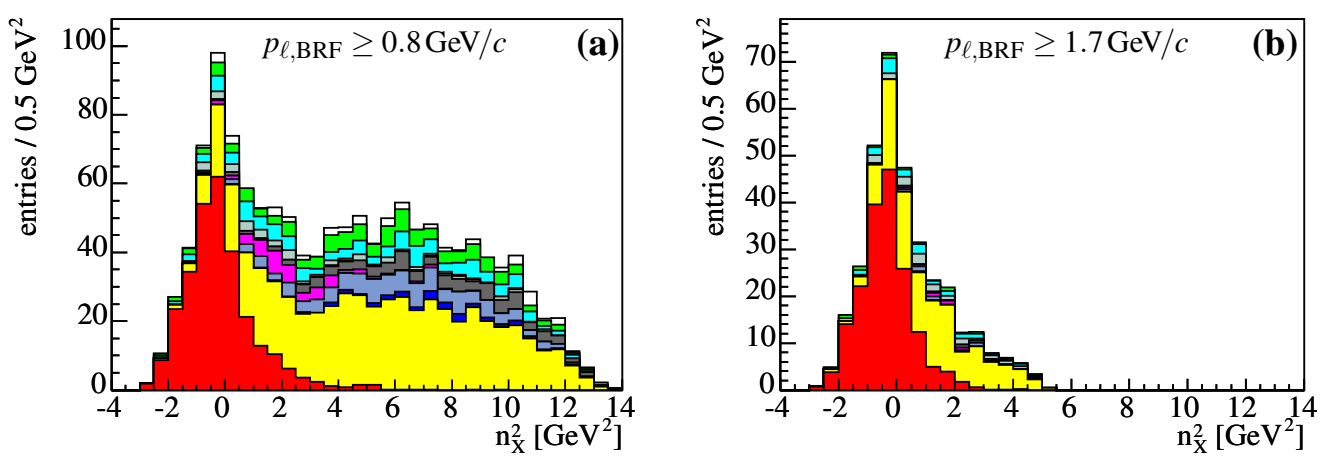

Figure 6.11: Breakdown of the residual background on the signal side for $p_{\ell, \mathrm{BRF}} \geq$ $0.8 \mathrm{GeV} / c$ (a) and $p_{\ell, \mathrm{BRF}} \geq 1.7 \mathrm{GeV} / c$ (b). The background contributions are $B \rightarrow X_{u} \ell v$ (ם), hadrons misidentified as leptons $(\square), B^{+, 0} \rightarrow X D^{+, 0} \rightarrow Y \ell^{+} v(\square), B^{+, 0} \rightarrow X D_{s}^{+} \rightarrow$ $Y \ell^{+} v(\square), B^{+, 0}\left(\rightarrow D_{s}^{+}\right) \rightarrow \tau^{+} \rightarrow \ell^{+}(\square)$, photon conversions $\gamma \rightarrow \ell^{+} \ell^{-}(\square)$, Dalitz de-

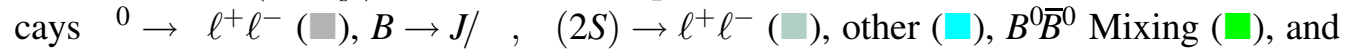
mistagged $B_{\text {reco }}$ candidates $(\square)$.

Leptons from $\mathbf{D}$ and $\mathbf{D}_{\mathbf{s}}$ Meson Decays $D$ and $D_{s}$ mesons decay into leptons with branching fractions of several percent. However, assuming a $B_{\text {reco }}$ containing a $\bar{b}$ quark, only mesons containing a $\bar{c}$ quark produce a lepton with negative charge and thus the correct charge correlation. Therefore, only $D$ or $D_{s}$ mesons produced at the so-called uppervertex, through the decay of the primary $W \rightarrow s \bar{c}$, as illustrated in figure 6.13, contribute to this background.

The branching fractions of decays $B^{0,+} \rightarrow D^{0,+} X$ have been measured by the BABAR collaboration [87]. Together with the branching fractions for semileptonic $D$-meson decays [88], this results in

$$
\begin{aligned}
& \mathcal{B}\left(B^{+} \rightarrow X D^{+} \rightarrow Y \ell^{+} v\right)=(2.5 \pm 0.5) \% \cdot(16.13 \pm 0.39) \%=(0.40 \pm 0.08) \%, \\
& \mathcal{B}\left(B^{0} \rightarrow X D^{+} \rightarrow Y \ell^{+} v\right)=(2.3 \pm 1.1) \% \cdot(16.13 \pm 0.39) \%=(0.37 \pm 0.18) \% \text {, } \\
& \mathcal{B}\left(B^{+} \rightarrow X D^{0} \rightarrow Y \ell^{+} v\right)=(8.6 \pm 0.7) \% \cdot(6.46 \pm 0.21) \%=(0.56 \pm 0.05) \% \text {, } \\
& \mathcal{B}\left(B^{0} \rightarrow X D^{0} \rightarrow Y \ell^{+} v\right)=(8.1 \pm 1.5) \% \cdot(6.46 \pm 0.21) \%=(0.52 \pm 0.10) \% \text {. }
\end{aligned}
$$

The scaling factors applied to our MC simulation vary between 0.71 and 1.09.

The branching fractions of decays $B^{0,+} \rightarrow D_{s}^{+} X$ are reported in ref. [87]. The total semileptonic branching fraction of $D_{s}$ mesons, however, is unmeasured. It can be estimated from the $D$ meson results using the lifetime ratio of $D$ and $D_{s}$ mesons assuming equal decay rates [89]. For lifetimes $\tau_{D^{0}}=(410.1 \pm 1.5) \mathrm{ps}, \tau_{D_{s}}=(500 \pm 7) \mathrm{ps}$ [42], and $\mathcal{B}\left(D^{0} \rightarrow\right.$ $X \ell v)=6.46 \pm 0.21 \%$ we obtain

$$
\mathcal{B}\left(D_{s}^{+} \rightarrow X \ell^{+} v\right)=(7.88 \pm 0.28) \% .
$$

In total we obtain

$$
\begin{aligned}
& \mathcal{B}\left(B^{+} \rightarrow X D_{s}^{+} \rightarrow Y \ell^{+} v\right)=\left(7.9_{-1.2}^{+1.5}\right) \% \cdot(7.88 \pm 0.28) \%=\left(0.62_{-0.10}^{+0.12}\right) \%, \\
& \mathcal{B}\left(B^{0} \rightarrow X D_{s}^{+} \rightarrow Y \ell^{+} v\right)=\left(10.3_{-1.8}^{+2.1}\right) \% \cdot(7.88 \pm 0.28) \%=\left(0.81_{-0.14}^{+0.17}\right) \% \text {. }
\end{aligned}
$$



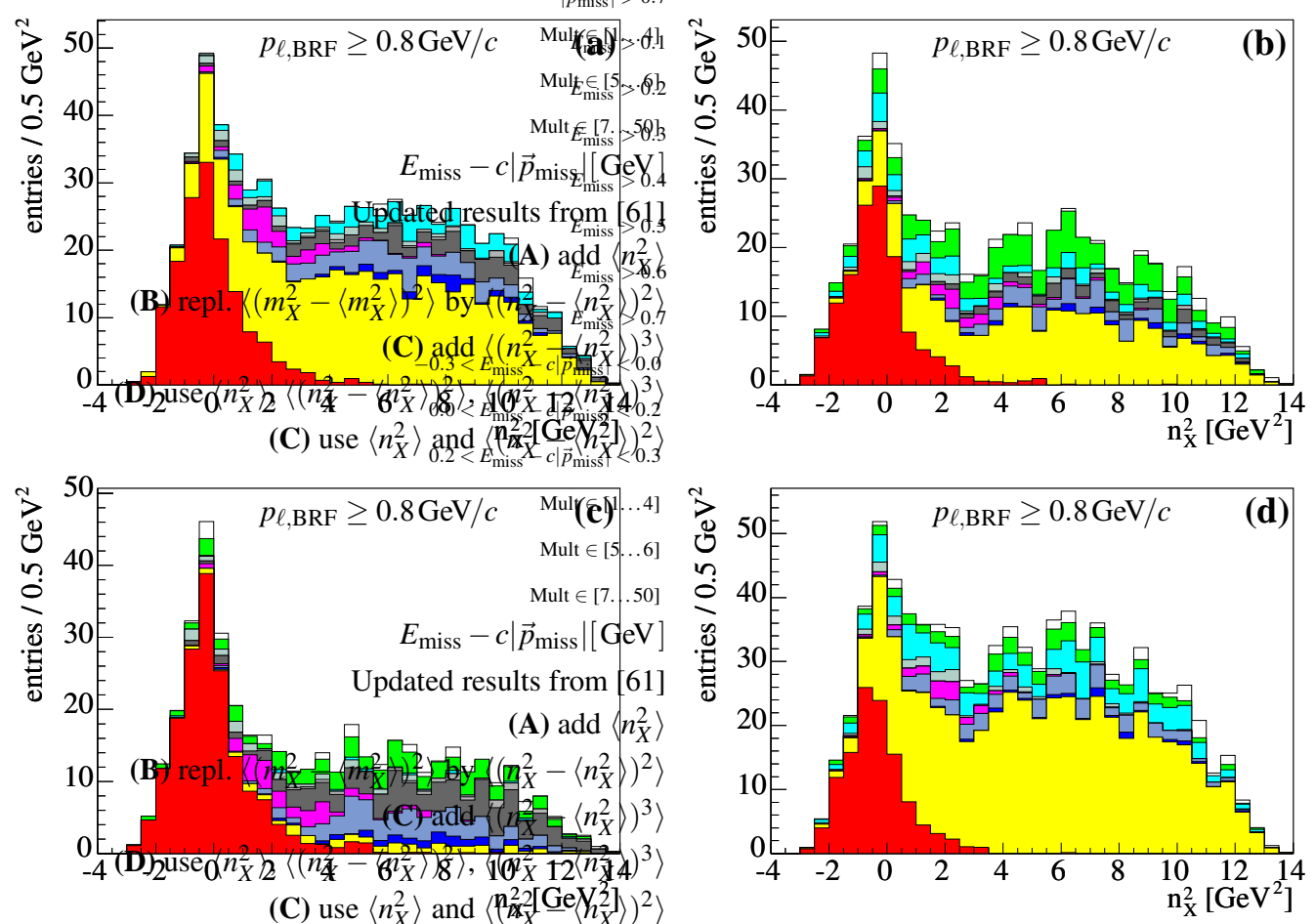

Figure 6.12: Breakdown of the residual background for $p_{\ell, \mathrm{BRF}} \geq 0.8 \mathrm{GeV} / c$ for subsets of the MC dataset: neutral $B$ mesons (a), charged $B$ mesons (b), electrons (c), and muons (d). The background contributions are $B \rightarrow X_{u} \ell v(\square)$, hadrons misidentified as leptons $(\square), B^{+, 0} \rightarrow X D^{+, 0} \rightarrow Y \ell^{+} v(\square), B^{+, 0} \rightarrow X D_{s}^{+} \rightarrow Y \ell^{+} v(\square), B^{+, 0}(\rightarrow$ $\left.D_{s}^{+}\right) \rightarrow \tau^{+} \rightarrow \ell^{+}(\square)$, photon conversions $\gamma \rightarrow \ell^{+} \ell^{-}(\square)$, Dalitz decays $\pi^{0} \rightarrow \gamma \ell^{+} \ell^{-}$ $(\square), B \rightarrow J / \psi, \psi(2 S) \rightarrow \ell^{+} \ell^{-}(\square)$, other $(\square), B^{0} \bar{B}^{0}$ Mixing ( $\square$ ), and mistagged $B_{\text {reco }}$ candidates $(\square)$.
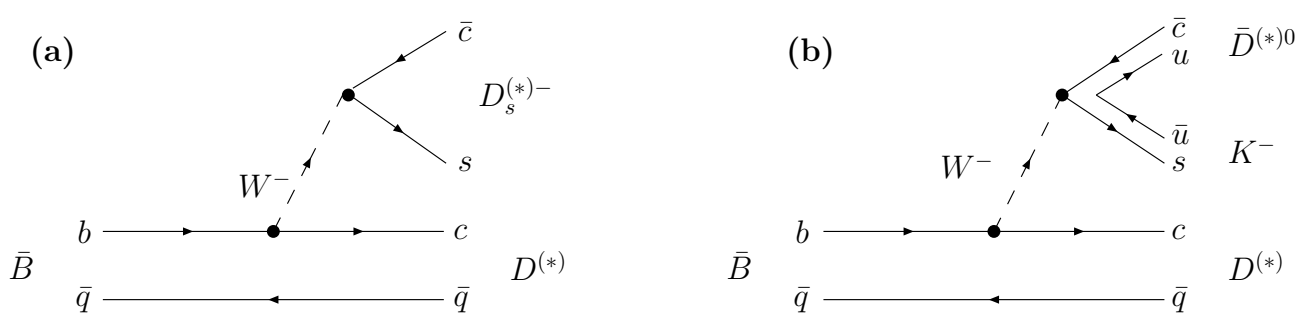

Figure 6.13: Two possible Feynman graphs for the production of right-flavored of $D_{s}^{(*)-}$ (a) or $\bar{D}^{(*)}$ (b) mesons at the so-called upper-vertex. These mesons can produce a lepton with the correct charge correlation w. r. t. the $B_{\text {reco }}$. 
The scaling factors applied to our MC simulation are 0.753 and 1.035 for $B^{+}$and $B^{0}$, respectively.

$\tau$ Decays $\tau$ leptons may appear in the selected event sample via leptonic or semileptonic decays of the signal $B$ meson directly, or via the decay of intermediate $D_{s}$ mesons. The leptonic decay $B \rightarrow \tau v$ is not considered due to its small branching fraction of $(1.8 \pm 0.7)$. $10^{-4}$ [93] and the expected very low selection efficiency of the full event reconstruction.

Semileptonic decays to $\tau$ leptons lead to right-charged leptons through the subsequent decay of the $\tau$ to electrons or muons. These decays $B \rightarrow D^{(*)} \tau \nu$ have been measured recently by the Belle and $B A B A R$ collaborations $[90,91]$. To scale our MC simulation, we use the sum of the measured branching fractions to $D$ and $D^{*}$ constrained to isospin symmetry reported in [91],

$$
\begin{aligned}
\mathcal{B}\left(B^{0} \rightarrow X \tau^{+} v\right) & =(0.86 \pm 0.27) \%+(1.62 \pm 0.33) \% & & =(2.48 \pm 0.18) \% \\
\mathcal{B}\left(B^{+} \rightarrow X \tau^{+} v\right) & =\tau_{B^{+}} / \tau_{B^{0}} \times \mathcal{B}\left(B^{0} \rightarrow X \tau^{+} v\right) & & =(2.66 \pm 0.18) \%
\end{aligned}
$$

Together with the average branching fractions of $\tau$ leptons to electrons and muons [42],

$$
\mathcal{B}\left(\tau^{+} \rightarrow \ell^{+} \bar{v}_{\tau} v_{\ell}\right)=(17.6 \pm 0.04) \%,
$$

we obtain

$$
\begin{aligned}
\mathcal{B}\left(B^{0} \rightarrow X \tau^{+} v \rightarrow \ell^{+} Y\right) & =(0.44 \pm 0.03) \%, \\
\mathcal{B}\left(B^{+} \rightarrow X \tau^{+} v \rightarrow \ell^{+} Y\right) & =(0.47 \pm 0.03) \%,
\end{aligned}
$$

where $\ell^{+}$is either $e^{+}$or $\mu^{+}$. The scaling factors are 0.9 and 0.83 , respectively. However, due to the three neutrinos in the final state for these decays, much higher values for $E_{\text {miss }}-$ $c\left|\vec{p}_{\text {miss }}\right|$ are expected, thus this is no relevant background source.

$\tau$ leptons from decays of $D_{s}$ mesons occur with branching fractions of

$$
\begin{aligned}
\mathcal{B}\left(B^{+} \rightarrow D_{s}^{+} \rightarrow \tau^{+} \rightarrow \ell^{+}\right) & =\left(0.09_{-0.02}^{+0.03}\right) \% \\
\mathcal{B}\left(B^{0} \rightarrow D_{s}^{+} \rightarrow \tau^{+} \rightarrow \ell^{+}\right) & =\left(0.12_{-0.03}^{+0.04}\right) \%,
\end{aligned}
$$

determined from eq. (6.34) and eq. (6.36) and $\mathcal{B}\left(D_{s}^{+} \rightarrow \tau^{+} v_{\tau}\right)=(6.4 \pm 1.5) \%$ [42].

Leptons from $J / \psi$ and $\psi(2 S)$ Decays Contributions to the background occur from $J / \psi$ and $\psi(2 S)$ decays into two leptons, if only the right-charged one is measured and the other one escapes the detector acceptance. The average branching fractions are $\mathcal{B}(J / \psi \rightarrow$ $\left.\ell^{+} \ell^{-}\right)=(5.94 \pm 0.06) \%$ and $\mathcal{B}\left(\psi(2 S) \rightarrow \ell^{+} \ell^{-}\right)=(0.733 \pm 0.042) \%$ [42]. Combining these with $\mathcal{B}(B \rightarrow J / \psi X)=(1.094 \pm 0.032) \%$ and $\mathcal{B}(B \rightarrow \psi(2 S) X)=(0.307 \pm 0.021) \%$ [42] results in

$$
\begin{aligned}
\mathcal{B}\left(B \rightarrow J / \psi \rightarrow \ell^{+} \ell^{-}\right) & =(6.46 \pm 0.20) \times 10^{-4} \text { and } \\
\mathcal{B}\left(B \rightarrow \psi(2 S) \rightarrow \ell^{+} \ell^{-}\right) & =(0.226 \pm 0.017) \times 10^{-4},
\end{aligned}
$$

respectively. This is only a minor contribution to the total background. The scaling factors for the MC simulation are 1.07 and 0.98 . respectively. 
$\mathbf{B}^{0} \overline{\mathbf{B}}^{0}$ Oscillations If either the signal $B$ or the $B_{\text {reco }}$ oscillates before it decays, the chargeflavor correlation with the lepton is inverted. Thus, $D$ mesons produced at the so-called lower-vertex, can produce a right-charged lepton via its semileptonic decay, $B \rightarrow D(\rightarrow$ $Y \ell v) X$.

The inclusive branching fraction of $B$ mesons into $D$ mesons at the lower-vertex, that is direct $b \rightarrow c$ or $\bar{b} \rightarrow \bar{c}$ transitions is $\mathcal{B}\left(B \rightarrow\left(\bar{D}^{0}, D^{-}\right) X\right)=\left(84.3_{-4.2}^{+4.4}\right) \%$ [42]. Subtracting the semileptonic signal decays $B \rightarrow X_{c} \ell v$ with $\mathcal{B}\left(B \rightarrow X_{c} \ell v\right)=(10.75 \pm 0.16) \%$ [24], results in

$$
\mathcal{B}\left(B^{0} \rightarrow\left(\bar{D}^{0}, D^{-}\right) X\right)=\left(62.8_{-4.19}^{+4.42}\right) \%
$$

In combination with the average semileptonic $D$ meson branching fraction [88],

$$
\mathcal{B}\left(D^{+, 0} \rightarrow X \ell^{+} v\right)=(11.30 \pm 0.22) \% .
$$

we estimate

$$
\mathcal{B}\left(B^{0} \rightarrow\left(\bar{D}^{0}, D^{-}\right) X \rightarrow Y \ell^{-} v\right)=\left(7.10_{-0.49}^{+0.52}\right) \% .
$$

The scaling factor we need to apply is $0.80 \pm 0.06$.

In addition to the branching fractions of the processes discussed above, also the mixing probability of $B^{0}$ mesons contribute to the amount of these background decays. In our MC simulation, a mixing probability of $\chi_{d, \mathrm{MC}}=0.181$ is implemented. The measured world average is $\chi_{d \text {,data }}=0.188 \pm 0.002$ [94], which is factor $1.04 \pm 0.01$ larger. As these $4 \%$ are small compared to the $20 \%$ correction from the branching fractions and fully covered by the uncertainty on the scaling factor, we do not apply an additional scaling factor.

Misidentified Hadrons A large contribution to the residual background originates from pions (kaons and protons contribute only marginally) misidentified as muons. This background is nearly negligible for the electron sample of the data, as the electron selector has a very low misidentification rate, as discussed above (cf. figure 6.12 (c) vs. (d)). The difference between selection efficiencies in data and simulation is corrected for in MC. The uncertainty on this so-called tweaking method is estimated by varying the efficiencies within the statistical uncertainties of the control sample. The procedure is described in more detail in section 7.1.4.

Semileptonic Decays to Charmless Final States Semileptonic decays $B \rightarrow X_{u} \ell v$ into light quarks contribute to the background, especially at higher lepton momenta. A world average has been calculated from inclusive $\left|V_{u b}\right|$ measurements [92] within the BLNP framework [95] to

$$
\mathcal{B}\left(B \rightarrow X_{u} \ell v\right)=(2.29 \pm 0.34) \cdot 10^{-3} .
$$

It is connected with a relatively large uncertainty of $15 \%$, which propagates into our scaling factor of $1.057 \pm 0.157$. 
Mistagged Events $\quad B_{\text {reco }}$ candidates which are completely misreconstructed are subtracted using the $m_{\mathrm{ES}}$ sideband, as they do not peak in $m_{\mathrm{ES}}$ at the $B$-meson mass. However, if only a pion, preferably with low momentum, is interchanged between the hadronic system and the $B_{\text {reco }}$, the $m_{\mathrm{ES}}$ of the candidate has most likely a value at the $B$-meson mass, thus these candidates peak in $m_{\mathrm{ES}}$. If a pion with the same charge is interchanged, no additional background category enters. If a charged pion is exchanged with a neutral one or with a pion with the opposite charge, the charge of the $B_{\text {reco }}$ is as well misreconstructed. MC studies show that true neutral $B_{\text {reco }}$ candidates are reconstructed with a probability of about $17.8 \%$ as a charged $B_{\text {reco }}$ candidate while only $4.84 \%$ of all charged $B_{\text {reco }}$ candidates are reconstructed as neutral candidates. Then, the same arguments as in the case of $B^{0} \bar{B}^{0}$ oscillations become valid, that is that $D$ mesons from direct $b \rightarrow c$ transitions will not be rejected by the charge correlation with the lepton. However, this category contributes only very little to the total residual background.

\subsubsection{Verification of the Background Description}

Pure background distributions can be selected by requiring the wrong correlation between charge and flavor of the lepton and the $B_{\text {reco }}$. On this sample the overall normalization as well as the shape of the measured background distributions can be checked.

Due to $B^{0} \bar{B}^{0}$ oscillations, this so-called wrong-sign sample of neutral $B$ mesons may contain signal decays. Thus this test is performed in divided samples $B^{+} B^{-}$with pure background distributions and $B^{0} \bar{B}^{0}$ with an additional signal component.

A $\chi^{2}$ fit of the combined background histogram to the measured data distribution is performed. A single fit parameter $s_{\mathrm{bg}, \text { scale }}$, which is the relative correction to the normalization derived from the number of $B_{\text {reco }}$ candidates, is extracted. It is compatible with unity in all lepton momentum ranges. The $\chi^{2}$ values of the fit are reasonable w.r.t. the number of degrees of freedom of the fit. Four spectra for the $B^{+} B^{-}$portion of the dataset are shown exemplarily in figure 6.14. The corresponding plots for neutral $B$ mesons are shown in figure 6.15. In our MC sample a mixing probability of $\chi_{d}=0.181$ is implemented. Performing the study for electrons and muons separately also yields results for $s_{\mathrm{bg}, \mathrm{scale}}$ compatible with one and good $\chi^{2}$ values.

\subsubsection{Determination of Background Subtraction Weights}

As the moments of the measured $n_{X}^{2}$ spectrum are determined as a weighted mean rather than from a binned distribution, which will be discussed later in section 6.8, $n_{X}^{2}$ dependent background subtraction weights are determined. Therefore, for each cut on $p_{\ell, \mathrm{BRF}}$, the measured $n_{X}^{2}$ spectrum together with the combinatorial and residual background is extracted, see figure 6.16 left column. The background subtraction weights are determined as

$$
w_{i}\left(n_{X}^{2}\right)=\frac{N_{\text {total }}\left(n_{X}^{2}\right)-N_{\mathrm{bg}}\left(n_{X}^{2}\right)}{N_{\text {total }}\left(n_{X}^{2}\right)},
$$

where $N_{\text {total }}$ is the measured number of events in a certain $n_{X}^{2}$-bin and $N_{\text {bg }}$ the sum of combinatorial and residual background in the same bin. These binned weights are fitted with a fourth order polynomial to get continuous weights in $n_{X}^{2}$, see figure 6.16 right column. In 

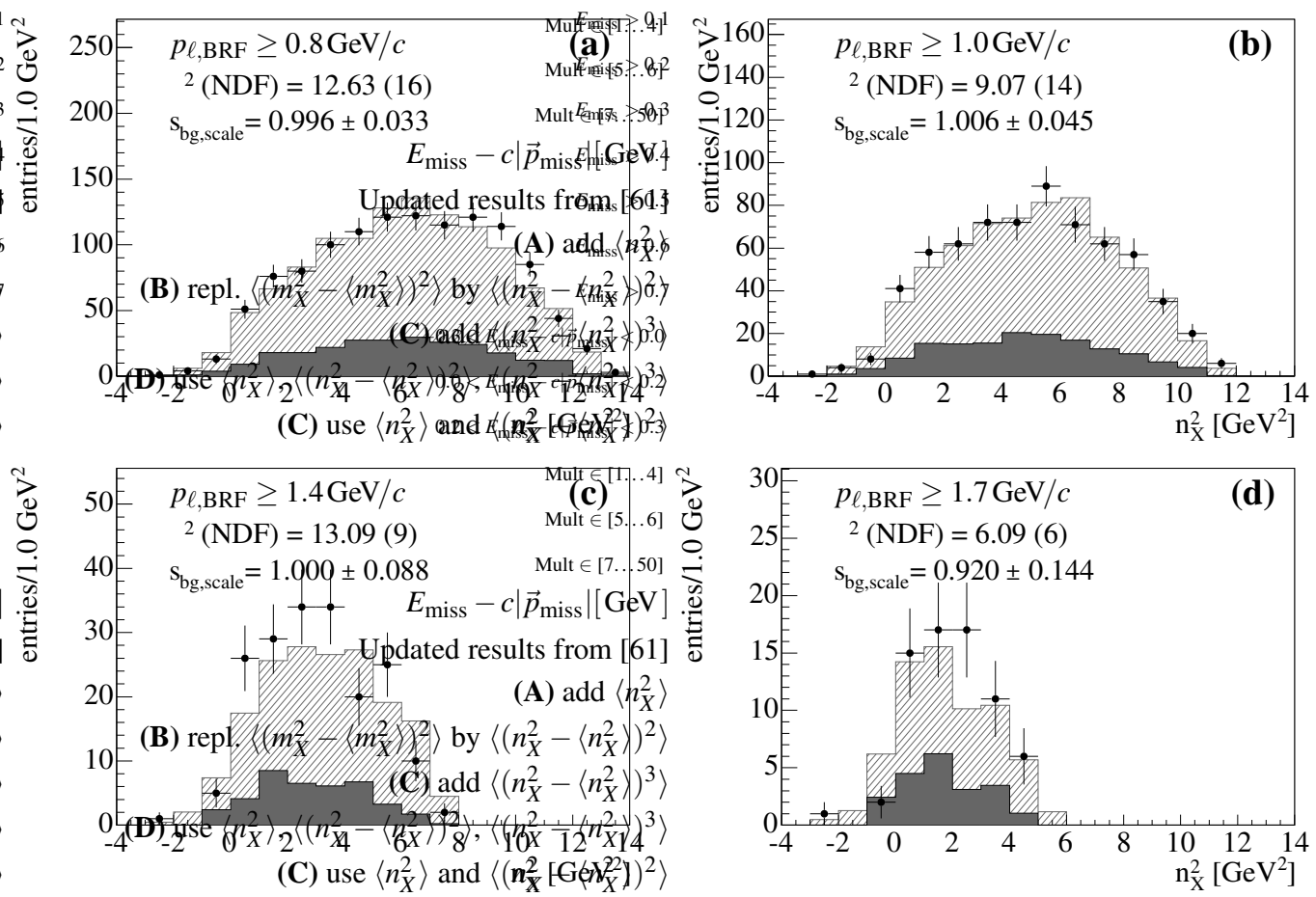

Figure 6.14: $n_{X}^{2}$ spectra of events with the wrong charge correlation between lepton and $B_{\text {reco }}$ for charged $B$ mesons and different cuts on $p_{\ell, \mathrm{BRF}}$ as written in the plots. This sample contains only background. The histograms are: observed spectrum in data $(\bullet$ with error bars), combinatorial background extracted from the $m_{\mathrm{ES}}$ sideband in data ( $\square$ ), and residual background extracted from MC (hatched histogram). 

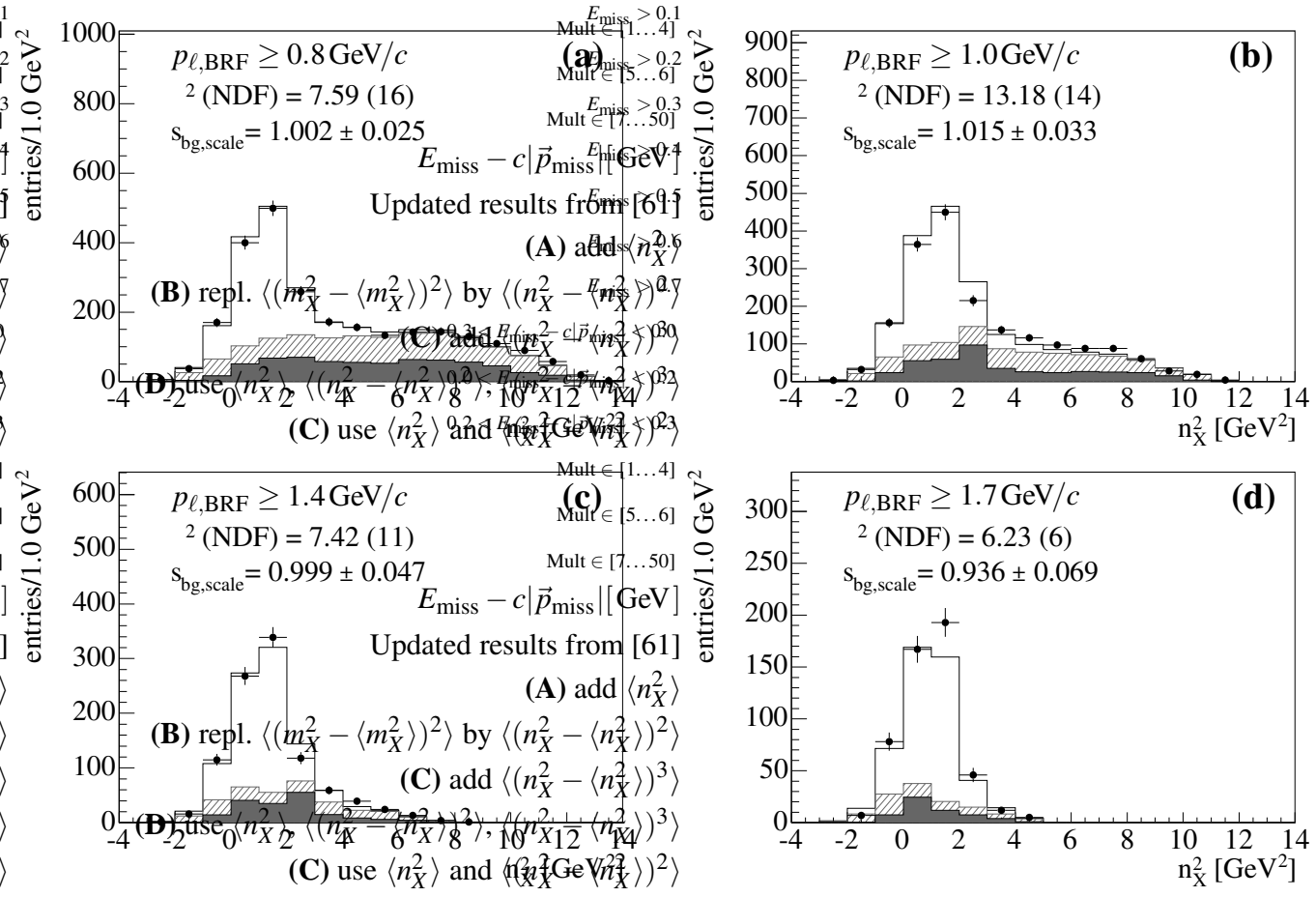

Figure 6.15: $n_{X}^{2}$ spectra of events with the wrong charge correlation between lepton and $B_{\text {reco }}$ for neutral $B$ mesons and different cuts on $p_{\ell, \mathrm{BRF}}$ as written in the plots. This sample contains background and signal events due to $B^{0} \bar{B}^{0}$ mixing (mixing probability in $\mathrm{MC}$ is $\left.\chi_{d}=0.181\right)$. The histograms are: observed spectrum in data ( $\bullet$ with error bars), combinatorial background extracted from the $m_{\mathrm{ES}}$ sideband in data ( $\square$ ), residual background extracted from MC (hatched histogram), and signal decays as extracted from MC ( $\square$ ). 
the right tail of the $n_{X}^{2}$ spectrum (e. g. for $n_{X}^{2}>9 \mathrm{GeV}^{2}$ in figure 6.16 (b)), where mainly background contributes, the polynomial is replaced with a constant at the minimum of the polynomial fit, which is zero or of the order of 0.03 to 0.05 . Setting all weights to zero in this region is always covered by the uncertainties of the weight function. The factors $w_{i}$ define the probability for an event with a certain value of $n_{X}^{2}$ to be a signal event.

\subsection{Extraction of Hadronic Moments}

This section will describe the formalism used to extract the hadronic moments $\left\langle n_{X}^{k}\right\rangle$ ( $k=$ $2,4,6)$. These moments are measured as a function of the lower cut on the lepton momentum in the $B$-meson rest-frame $p_{\ell, \mathrm{BRF}}$. Investigations on MC simulations reveal a misreconstruction of the moments of up to $40 \%$ (see section 6.8.1). A so-called calibration method, which has proven reliable results in previous measurements of hadronic-mass moments [2, 60,61], is tested and adjusted to the requirements of this analysis (section 6.8.2). It leaves a small bias in the percent region, which is discussed and corrected for, see section 6.8.3. Furthermore, the statistical uncertainties and correlations of the moments are derived in section 6.8.4, the formalism to calculate central moments is discussed in section 6.8 .5 .

\subsubsection{Situation Before Any Correction}

Even though the kinematic fit applied to the complete event $e^{+} e^{-} \rightarrow \Upsilon(4 S) \rightarrow B \bar{B}$ already reduces the observed bias in the reconstructed value of $n_{X}^{2}$, the precise determination of the moments needs further correction. We observe that the moments with a lower cut on the lepton momentum are measured about $10 \%$ to $30 \%$ too low for $\left\langle n_{X}^{2}\right\rangle$ as illustrated in figure 6.17 (a). For the higher orders $\left\langle n_{X}^{4(6)}\right\rangle$ (plots (b) and (c)), the bias decreases from about $30 \%$ at high lepton momentum cuts to a few percent at low lepton momentum cuts. For the lowest cuts, the moments are even measured too large by a few percent. The moments binned in the lepton momentum give more insight into the characteristics of the misreconstruction, see figure 6.17 (d)-(f). Especially the moments $\left\langle n_{X}^{2}\right\rangle$ are measured too high at low lepton momenta up to $1.2 \mathrm{GeV} / c$, and too low at higher momenta.

Further studies reveal that this behavior is mainly caused by the decays $B \rightarrow X_{c} e v$ rather the ones including a muon, compare figure 6.18. While the latter are constantly measured too low, the moments of events including an electron are measured too large at low lepton momenta. One significant difference between the two lepton types is the enhanced probability for an electron to emit bremsstrahlung. To study this effect, the misreconstruction of the lepton momentum has been investigated, see figure 6.19. It shows that, while muon momenta are well reconstructed without significant bias and a width of about $25 \mathrm{MeV} / c$, especially low electron momenta are often misreconstructed by more than $200 \mathrm{MeV} / c$. Restricting the study presented above to those events with $\left|p_{\ell, \mathrm{BRF}}-p_{\ell, \mathrm{BRF} \text {,true }}\right|<0.2 \mathrm{GeV} / c$, we obtain a similar bias of the measured moments $\left\langle n_{X}^{k}\right\rangle$ for electrons and muons, as shown in figure 6.20 .

The observed positive bias in the hadronic moments for the events $B \rightarrow X_{c} e v$ has been found to be caused by the kinematic fit. The lepton momentum can only be fitted within the error matrix provided by the track fit, which does not include a possible misreconstruction 

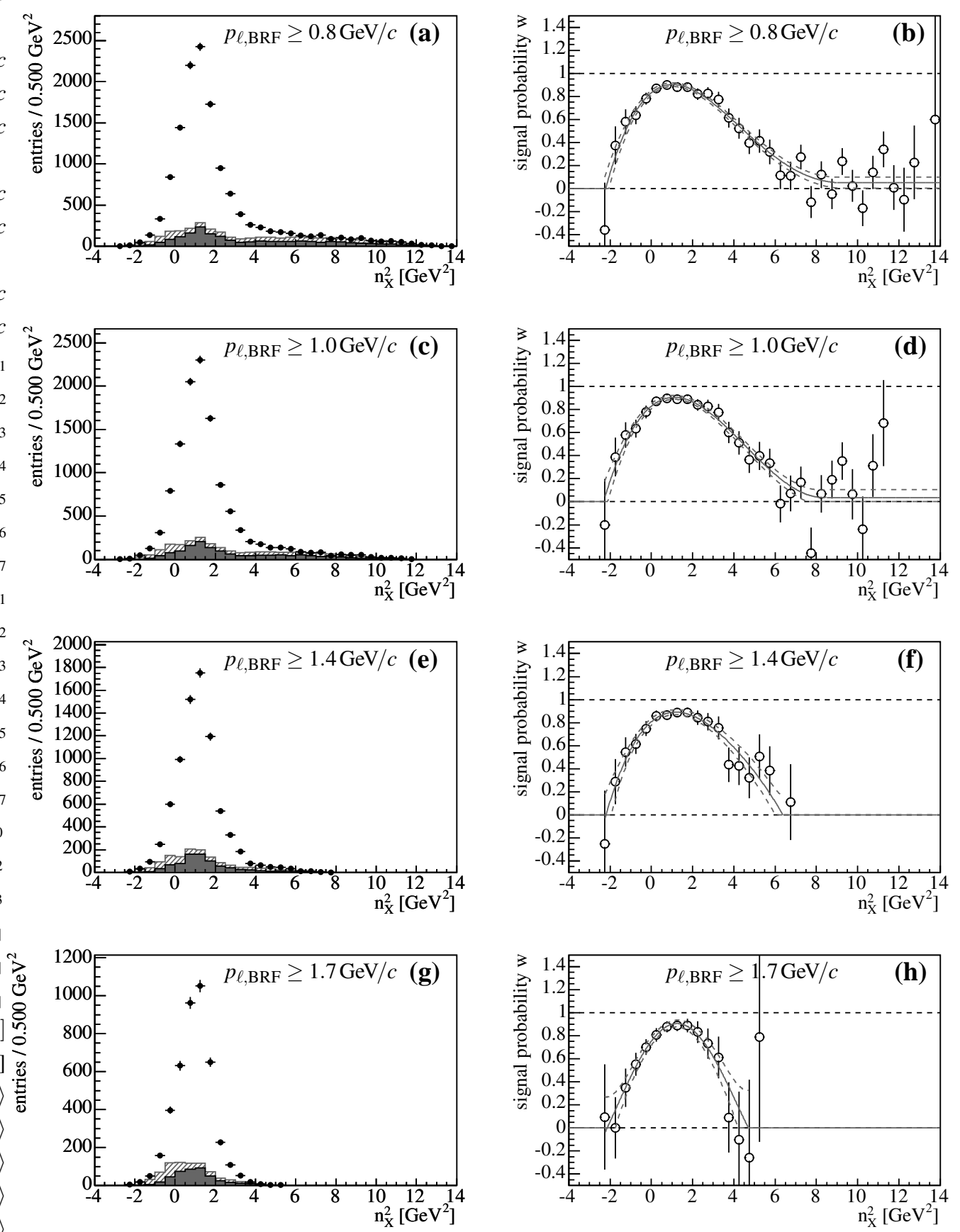

Figure 6.16: The left column shows the measured $n_{X}^{2}$ spectra in data $(\bullet)$ together with the extracted combinatorial ( $\square$ filled histogram) and residual (hatched histogram) background distributions for different cuts on $p_{\ell, \mathrm{BRF}}$. The corresponding background subtraction factors $w_{i}$ are shown in the right column together with the fit of a polynomial of fourth order (solid line). In the right tail of the $n_{X}^{2}$ spectrum the polynomial is replaced with a constant at the minimum of the polynomial fit. The uncertainties of the fit are shown as dashed lines. 

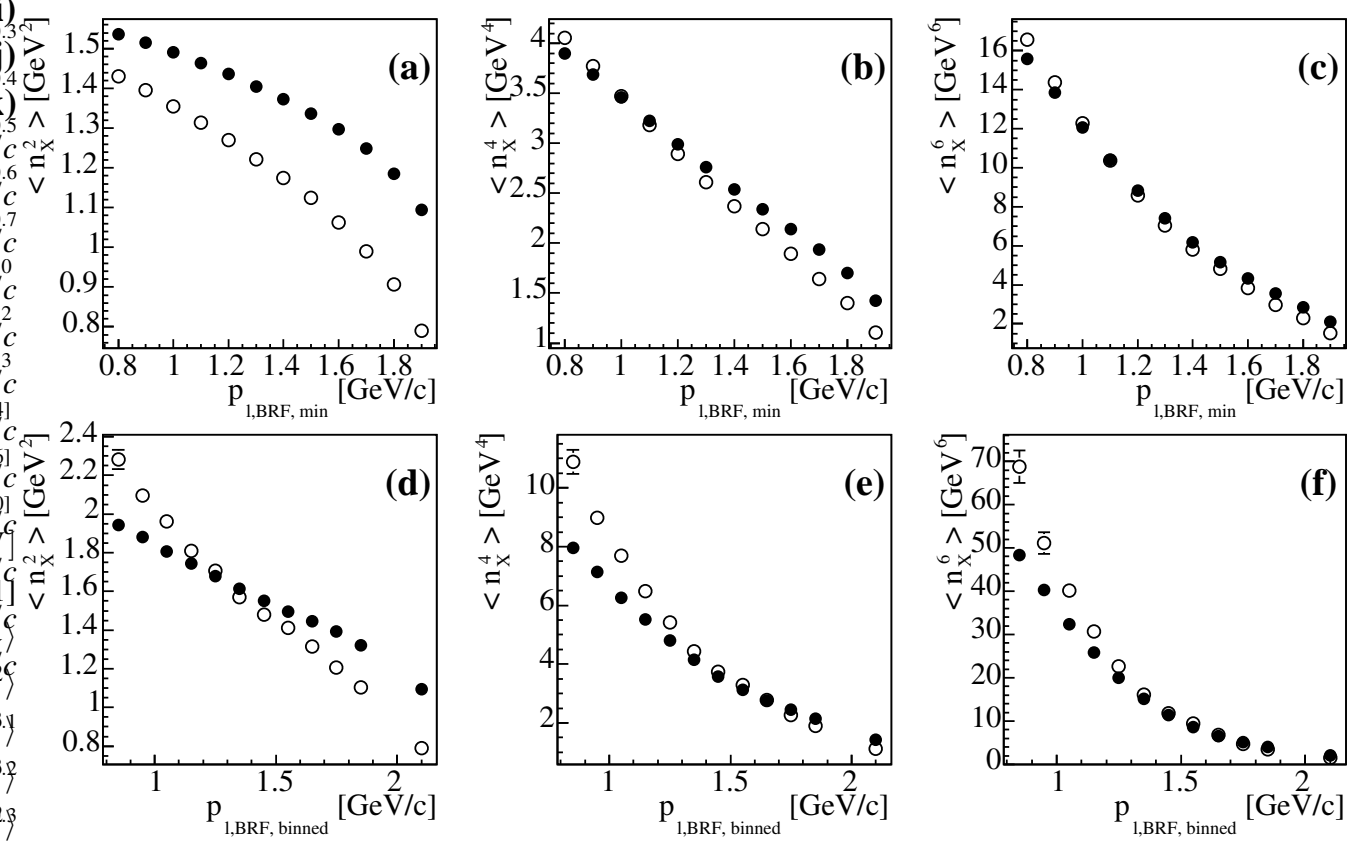

Figure 6.17: Moments $\left\langle n_{X}^{2(4 / 6)}\right\rangle$ determined in MC simulations as a functions of a lower cut on $p_{\ell, \mathrm{BRF}}(\mathrm{a}-\mathrm{c})$ or binned in $p_{\ell, \mathrm{BRF}}(\mathrm{d}-\mathrm{f})$. The moments on generator level $(\bullet)$ are compared to the ones measured in the selected event sample after the kinematic fit on generic MC (o).
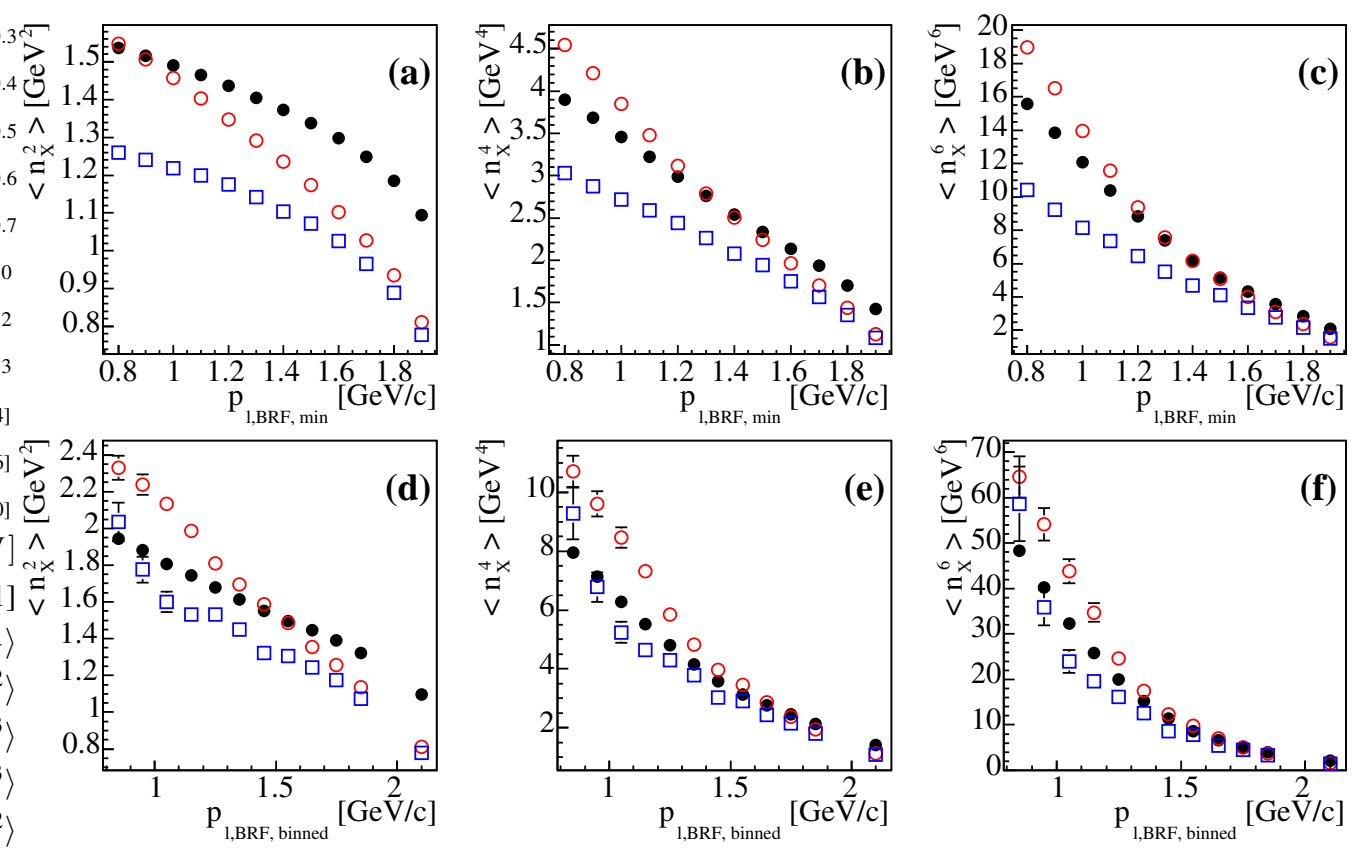

Figure 6.18: Moments $\left\langle n_{X}^{2(4 / 6)}\right\rangle$ determined in MC as functions of a lower cut on $p_{\ell, \mathrm{BRF}}$ (a-c) or binned in $p_{\ell, \mathrm{BRF}}(\mathrm{d}-\mathrm{f})$. The moments on generator level $(\bullet)$ are compared to the ones measured in the selected event sample for $B \rightarrow X_{c} e v(\circ)$ and $B \rightarrow X_{c} \mu \nu(\square)$. 

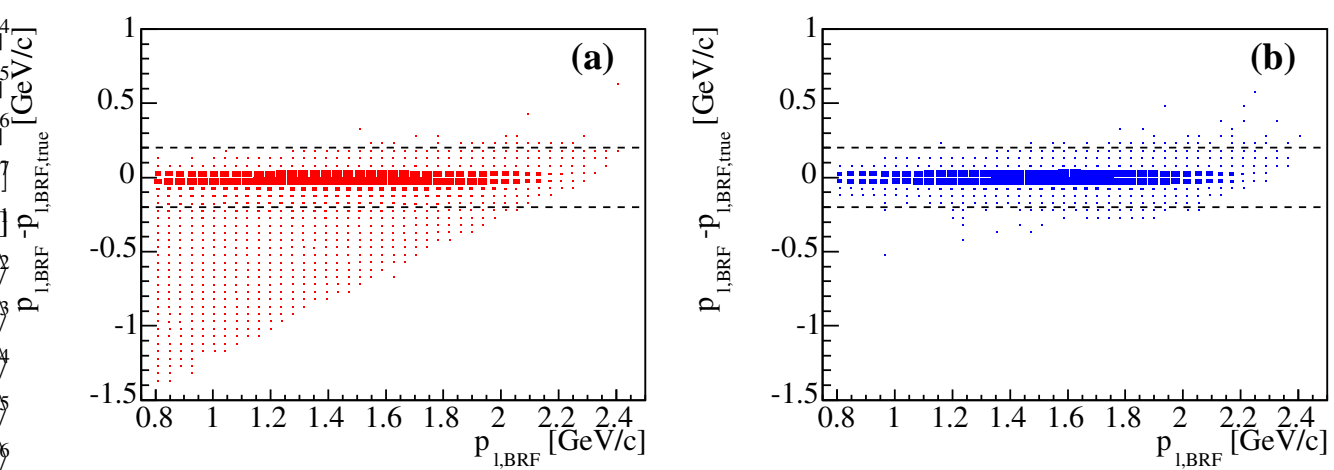

Figure 6.19: Momentum misreconstruction $p_{\ell, \mathrm{BRF}}-p_{\ell, \mathrm{BRF} \text {,true }}$ plotted vs. the measured momentum $p_{\ell, \text { BRF. }}$. Figure (a) shows the scatter plot for electrons, (b) for muons. The dashed lines indicate a requirement on the momentum reconstruction applied in figure 6.20 .
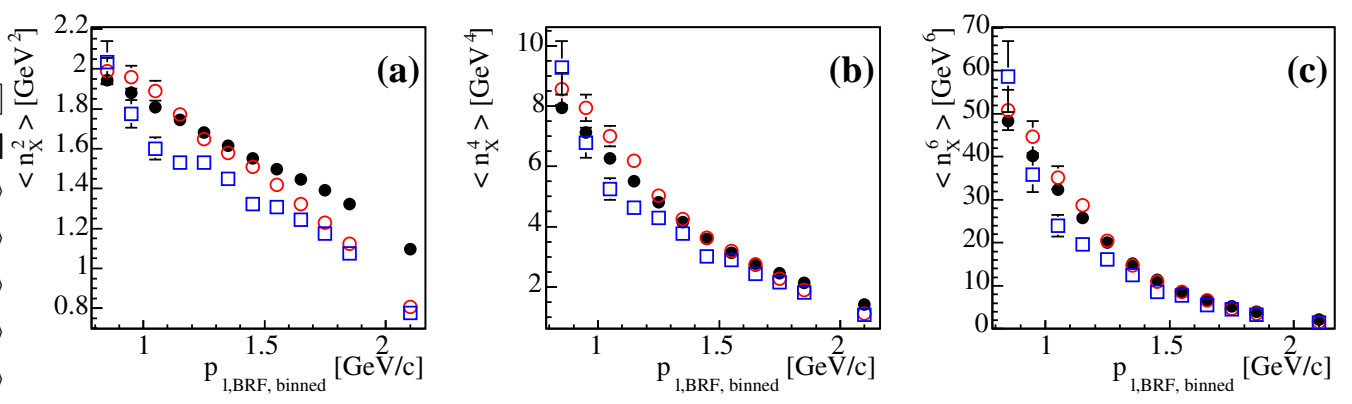

Figure 6.20: Moments $\left\langle n_{X}^{2(4 / 6)}\right\rangle$ determined in MC binned in $p_{\ell, \mathrm{BRF}}(\mathrm{a}-\mathrm{c})$. The moments on generator level $(\bullet)$ are compared to the ones measured in the selected event sample for $B \rightarrow X_{c} e v(\circ)$ and $B \rightarrow X_{c} \mu \nu(\square)$. The reconstructed lepton momentum is required to fulfill $\left|p_{\ell, \mathrm{BRF}}-p_{\ell, \mathrm{BRF}, \text { true }}\right|<0.2 \mathrm{GeV} / c$.

due to bremsstrahlung. However, the hadronic system has larger uncertainties and can therefore be changed by the fit to a larger amount. Thus, in cases where the constraints of the kinematic fit are not fulfilled initially due to a lepton momentum measured too low, the fit will increase the hadronic momentum and energy instead. This leads to moments of the distribution measured too large. Although this behavior is not optimal, we found that the correction method presented in the following can be adapted to correct for this different behavior for electrons and muons. These two samples are also studied in detail separately in data and no difference is found, giving confidence in the extraction method.

\subsubsection{Calibration Method}

The aim of the calibration method is to correct the measured moments of the selected data sample to the true moments, obtained without any restrictions on the available phase space and without misreconstruction due to FSR, which are modeled by the generator MC dataset. This is done via a linear correction function, which is applied event by event as a 
function of the measured $n_{X}^{2}$ and three additional variables. It is derived from the following linear approximation of the relation between true and measured moments in Monte Carlo events,

$$
n_{X, \text { reco }}^{k}=p_{0, k}+p_{1, k} \cdot n_{X, \text { true }}^{k} \quad \text { for } k=2,4,6 .
$$

The parameters of these functions are determined in the following way. The selected events of the Cocktail MC dataset are separated in bins of their lepton momentum, their total $X_{c}$ multiplicity, that is the total number of charged and neutral candidates used to build the $X_{c}$ system, and their measured $E_{\text {miss }}-c\left|\vec{p}_{\text {miss }}\right|$. The following bins are used, chosen according to regions with similar resolution,

- 12 bins of $0.1 \mathrm{GeV} / c$ in $p_{\ell, \mathrm{BRF}}$ from [0.8-0.9] GeV/c to [1.9-2.3] GeV/c,

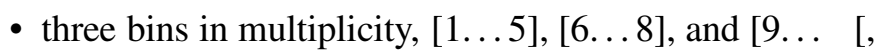

- and three bins in $E_{\text {miss }}-c\left|\vec{p}_{\text {miss }}\right|,[-0.2,0.0] \mathrm{GeV},[0.0,0.2] \mathrm{GeV}$, and $[0.2,0.3] \mathrm{GeV}$.

It turns out that the calibration graphs differ for electrons and muons, a direct consequence on what has been observed in section 6.8.1. Therefore, the number of bins is doubled and different calibration curves for events $B \rightarrow X_{c} \mu \nu$ and $B \rightarrow X_{c} e v$ are built. The $n_{X}^{2}$ spectrum of the events in each of these bins is divided into six bins of the true $n_{X}^{2}$, namely $[0.00,0.68,1.00,1.28,1.60,2.64,15.0] \mathrm{GeV}^{2}$. For each of these intervals the true mean on generator level $\left\langle n_{X, \text { true }}^{k}\right\rangle$ and the measured mean $\left\langle n_{X, \text { reco }}^{k}\right\rangle$ is determined. This gives six datapoints for each order $k=2,4,6$ which can be fitted by the linear function given in eq. (6.45). The linear relationship is found for all orders of $n_{X}^{2}$. Whenever one interval in $n_{X \text {.true }}^{2}$ has less than 20 measured entries, it is merged with its neighbor interval.

The binning in multiplicity and $E_{\text {miss }}-c\left|\vec{p}_{\text {miss }}\right|$ is motivated by differences of the reconstruction quality in different regions of these variables. An $X_{c}$ system with high multiplicity will be more frequently misreconstructed than a system with low multiplicity, as the probability that a particle escapes the detector acceptance rises. As $E_{\text {miss }}-c\left|\vec{p}_{\text {miss }}\right|$ is a measure for the lost or additional energy and momentum in an event, the same argument is valid here. The binning in $p_{\ell, \mathrm{BRF}}$ is not directly motivated in such a way, but studies concerning the dependence of the calibration curve parameters on $p_{\ell, \mathrm{BRF}}$ reveal a clear dependence. Thus this binning is as well used for calibrating.

The calibration then is performed on an event-by-event basis. For each event its measured $n_{X \text {,reco }}^{k}$ is calibrated towards a calibrated $n_{X, \text { calib }}^{k}$ via the inverse calibration curve,

$$
\begin{array}{cl}
n_{X, \text { calib }}^{k}=\frac{n_{X, \text { reco }}^{k}-p_{0, k}}{p_{1, k}} & \text { with } p_{0 / 1, k}=p_{0 / 1, k}\left(p_{\ell, \mathrm{BRF}}, E_{\mathrm{miss}}-c\left|\vec{p}_{\mathrm{miss}}\right|, \text { mult }\right) \\
& \text { and } k=2,4,6
\end{array}
$$

The moments $\left\langle n_{X}^{k}\right\rangle$ are then determined as the means of these calibrated values $n_{X, \text { calib }}^{k}$.

The difference in the calibration graphs for electrons and muons is especially apparent for the fitted offset $p_{0}$ of the calibration function for low lepton momenta as visible by comparing figure 6.21 and 6.22 . Here the calibration curves for $n_{X}^{2}$ are plotted separately for electrons and muons. The curves for the moments $\left\langle n_{X}^{4(6)}\right\rangle$ are shown in figure A.1 to A.4. 
To test whether this difference comes from the larger fraction of events with a FSR photon generated by PHOTOS in the electron sample, we measure calibration curves rejecting those events. The measured offsets for electrons and muons come closer but still a difference is present, which most likely is due to external bremsstrahlung in the detector. We extract the final result from the separated calibration curves, choosing the applied calibration curve for the determination of $n_{X \text {.calib }}^{k}$ according to the lepton type, and summing these calibrated values of the whole dataset to determine the moments. This avoids any corrections which could be necessary due to different fractions of $e$ and $\mu$ events in data and MC, respectively.
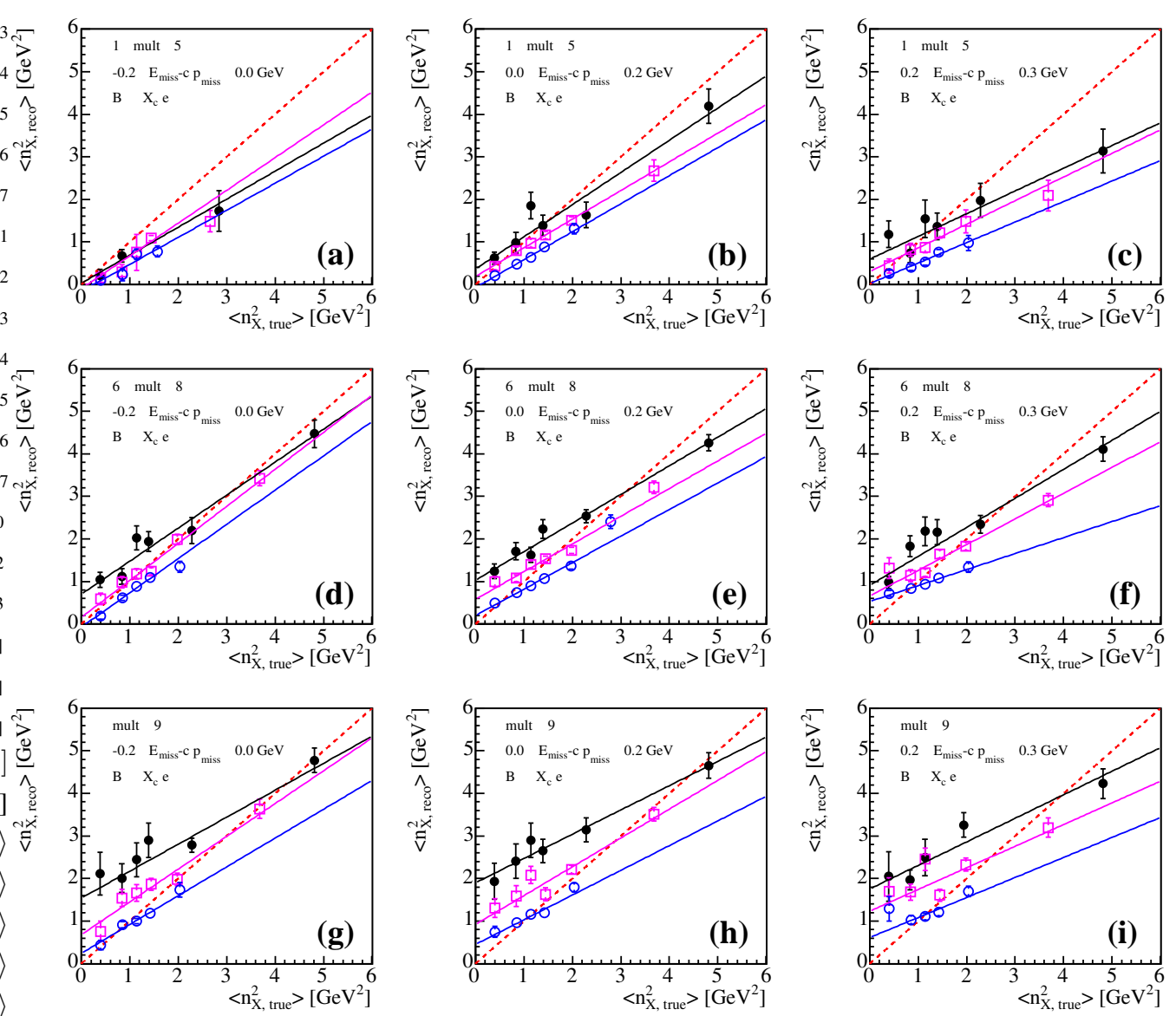

Figure 6.21: Examples of calibration curves for $\left\langle n_{X}^{2}\right\rangle$ in bins of multiplicity, $E_{\text {miss }}-$ $c\left|\vec{p}_{\text {miss }}\right|\left((\mathrm{a})\right.$-(i)), and $p_{\ell, \mathrm{BRF}}$ (color code), extracted for events $B \rightarrow X_{c} e v$. Shown are the extracted $\left\langle n_{X, \text { reco }}^{2}\right\rangle$ versus $\left\langle n_{X, \text { true }}^{2}\right\rangle$ in bins of $n_{X \text { true }}^{2}$ for $0.9<p_{\ell, \mathrm{BRF}} \leq 1.0 \mathrm{GeV} / c$ $(\bullet), 1.4<p_{\ell, \mathrm{BRF}} \leq 1.5 \mathrm{GeV} / c(\square)$, and $p_{\ell, \mathrm{BRF}} \geq 1.9 \mathrm{GeV} / c(\circ)$. The results of fits of linear functions are overlaid as solid lines. Reference lines with $\left\langle n_{X, \text { reco }}^{k}\right\rangle=\left\langle n_{X \text {,rue }}^{k}\right\rangle$ are superimposed (dashed lines). 

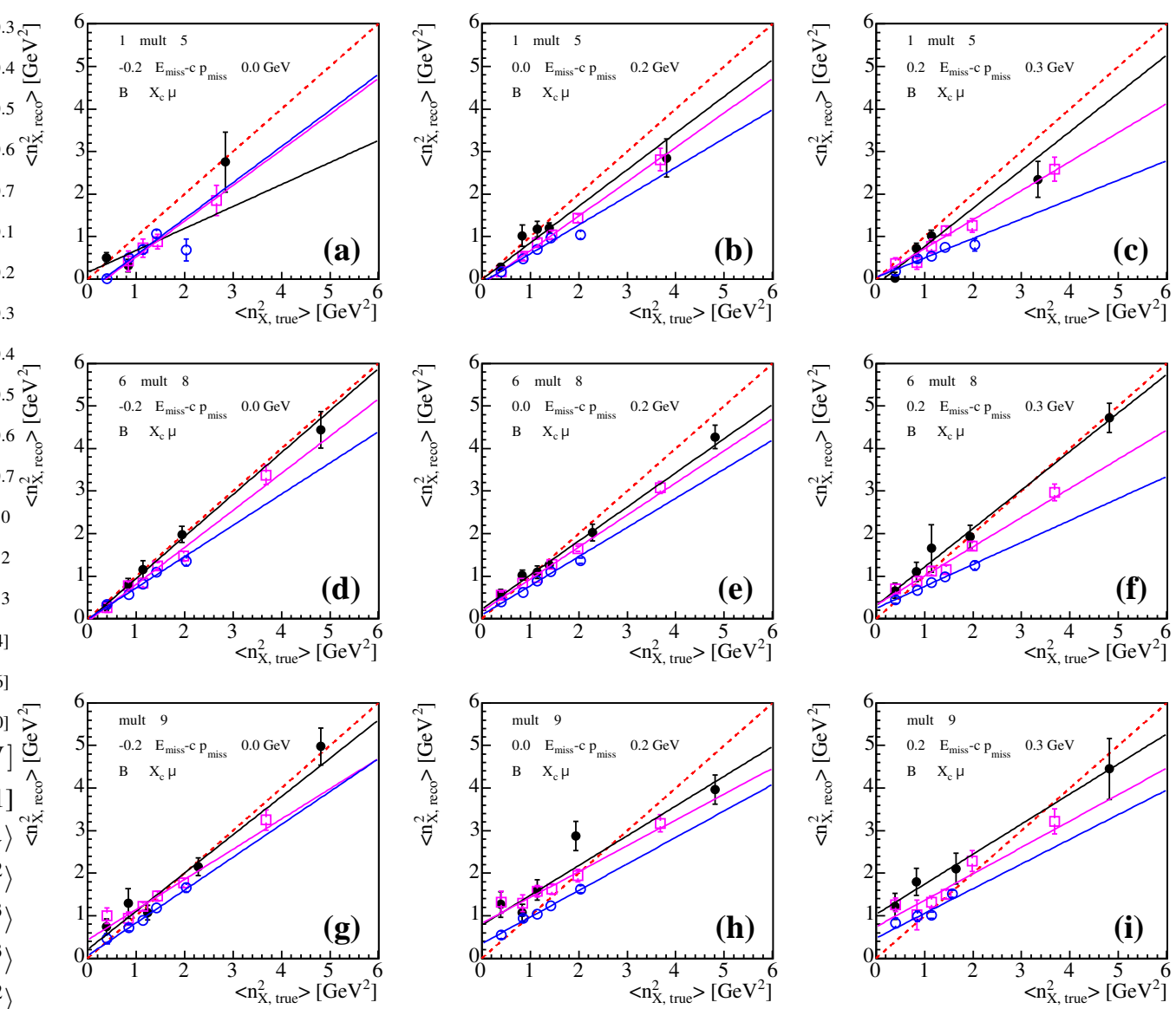

Figure 6.22: Examples of calibration curves for $\left\langle n_{X}^{2}\right\rangle$ in bins of multiplicity, $E_{\text {miss }}-$ $c\left|\vec{p}_{\text {miss }}\right|\left(\left(\right.\right.$ a)-(i)), and $p_{\ell, \mathrm{BRF}}$ (color code), extracted for events $B \rightarrow X_{c} \mu v$. Shown are the extracted $\left\langle n_{X \text {,reco }}^{2}\right\rangle$ versus $\left\langle n_{X \text {,true }}^{2}\right\rangle$ in bins of $n_{X \text { true }}^{2}$ for $0.9<p_{\ell, \mathrm{BRF}} \leq 1.0 \mathrm{GeV} / c$ $(\bullet), 1.4<p_{\ell, \mathrm{BRF}} \leq 1.5 \mathrm{GeV} / c(\square)$, and $p_{\ell, \mathrm{BRF}} \geq 1.9 \mathrm{GeV} / c(\circ)$. The results of fits of linear functions are overlaid as solid lines. Reference lines with $\left\langle n_{X, \text { reco }}^{k}\right\rangle=\left\langle n_{X \text {,true }}^{k}\right\rangle$ are superimposed (dashed lines). 

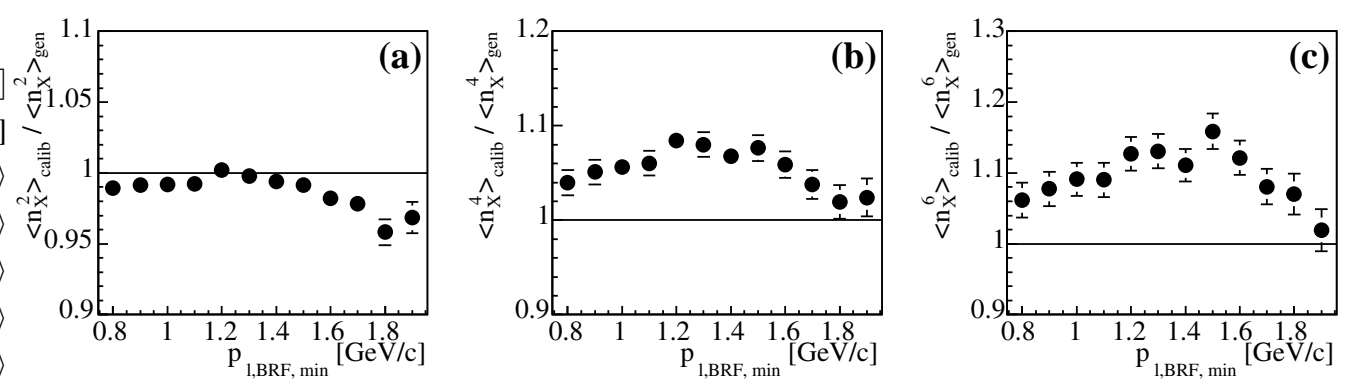

Figure 6.23: Bias after application of the calibration on simulated signal decays for the moments $\left\langle n_{X}^{2}\right\rangle$ (a), $\left\langle n_{X}^{4}\right\rangle$ (b), and $\left\langle n_{X}^{6}\right\rangle$ (c).

\subsubsection{Remaining Bias Correction}

Even though the calibration method described above already corrects most of the misreconstructed moments, there is a deviation remaining after this procedure. It is studied for the moments of the whole dataset, dropping the distinction between events containing an electron or muon. The observed bias is due to the imperfection of the calibration method itself, e. g. to small non-linearities in the calibration curves. Therefore, a set of correction factors is derived to eliminate this bias. For the extraction of the moments on data, these bias correction factors are derived on signal decays in generic $B \bar{B} \mathrm{MC}$,

$$
\left\langle n_{X}^{k}\right\rangle_{\text {calib }} \stackrel{\text { bias corr. }}{\longrightarrow} C \cdot\left\langle n_{X}^{k}\right\rangle_{\text {calib }} \quad \text { with } \quad C=\frac{\left\langle n_{X}^{k}\right\rangle_{\text {gen }}}{\left\langle n_{X}^{k}\right\rangle_{\text {calib }}} .
$$

It should be noted that the moments $\left\langle n_{X}^{k}\right\rangle_{\text {calib }}=\left\langle n_{X}^{k}\right\rangle_{\text {calib }}\left(p_{\ell, \mathrm{BRF}}\right)$ as well as the correction factors $C=C\left(p_{\ell, \mathrm{BRF}}\right)$ depend on the cut on the lepton momentum. Figure 6.23 shows this momentum dependence of the correction factors for the moments $\left\langle n_{X}^{k}\right\rangle, k=2,4,6$. While the bias for the moments $\left\langle n_{X}^{2}\right\rangle$ is in the order of a few percent, it is larger for the moments $\left\langle n_{X}^{4}\right\rangle$ and $\left\langle n_{X}^{6}\right\rangle$. Several investigations have been performed to minimize the observed bias after the calibration, however the analysis cuts do not have a great impact on the result.

\subsubsection{Statistical Uncertainties and Correlations}

The variance of the extracted moments related to the number of observed events is derived from the fact that a weighted mean is calculated,

$$
\begin{aligned}
V\left(\left\langle n_{X}^{k}\right\rangle\right) & =V\left(\frac{\sum_{i=1}^{N_{\text {evt }}} w_{i} n_{i}^{k}}{\sum_{i=1}^{N_{\text {evt }}} w_{i}}\right)=\frac{1}{\left(\sum_{i=1}^{N_{\text {evt }}} w_{i}\right)^{2}} \cdot V\left(\sum_{i=1}^{N_{\text {evt }}} w_{i} n_{i}^{k}\right) \\
& =\frac{\sum_{i=1}^{N_{\text {evt }}} w_{i}^{2}}{\left(\sum_{i=1}^{N_{\text {evt }}} w_{i}\right)^{2}} V\left(n^{k}\right)=\frac{\sum_{i=1}^{N_{\text {evt }}} w_{i}^{2}}{\left(\sum_{i=1}^{N_{\text {evt }}} w_{i}\right)^{2}}\left\langle n^{2 k}\right\rangle-\left\langle n^{k}\right\rangle^{2} .
\end{aligned}
$$

Here $n^{k}$ is the calibrated but unweighted value of $n_{X}^{k}$, the indices $X$ and calib are omitted for a better readability. The uncertainties arising from calibration and background weighting 
are evaluated separately as systematic effects. Thus, the treatment of the weights $w_{i}$ as constants is justified here.

As the moments are determined with a lower cut on the lepton momentum, moments with different cuts share a number of events and thereby are statistically correlated. For the calculation of central moments such as $\left\langle\left(n_{X}^{2}-\left\langle n_{X}^{2}\right\rangle\right)^{2}\right\rangle$ with their correct uncertainty and for the comparison with theory predictions, it is important to evaluate these correlations.

For the calculation of the correlation we use the fact that a larger sample of events (e.g. $p_{\ell, \mathrm{BRF}} \geq 0.8 \mathrm{GeV} / c$ which we call set $C$ ) can be splitted into two samples, one fully overlapping with the sample of another cut (e.g. $p_{\ell, \mathrm{BRF}} \geq 1.2 \mathrm{GeV} / c$ called set $B$ ) and one statistically independent $\left(p_{\ell, \mathrm{BRF}} \in[0.8,1.2[\mathrm{GeV} / c\right.$ called set $A)$,

$$
C=A \cup B \quad \text { and } \quad A \cap B=\varnothing .
$$

The correlation between moments with different cuts arises only from those events, which are shared between them. It is convenient to rewrite the definition of a moment calculated from a large data sample $C$,

$$
\begin{aligned}
\left\langle n^{k}\right\rangle_{C}=\frac{\sum_{i=1}^{N_{C}} w_{i} n_{i}^{k}}{\sum_{i=1}^{N_{C}} w_{i}}=\frac{\sum_{i=1}^{N_{A}} w_{i} n_{i}^{k}+\sum_{i=N_{A}+1}^{N_{C}} w_{i} n_{i}^{k}}{\sum_{i=1}^{N_{C}} w_{i}} \\
=\frac{\left\langle n^{k}\right\rangle_{A} \cdot \sum_{i=1}^{N_{A}} w_{i}+\left\langle n^{k}\right\rangle_{B} \cdot \sum_{i=N_{A}+1}^{N_{C}} w_{i}}{\sum_{i=1}^{N_{C}} w_{i}} .
\end{aligned}
$$

Taking this, we can write the covariance $\mathcal{C}$ between two moments of different datasets $A$ and $C$ as

$$
\begin{aligned}
\mathcal{C}\left(\left\langle n^{k}\right\rangle_{A},\left\langle n^{l}\right\rangle_{C}\right) & \stackrel{(6.50)}{=} \mathcal{C}\left(\left\langle n^{k}\right\rangle_{A}, \frac{\left\langle n^{l}\right\rangle_{A} \cdot \sum_{i=1}^{N_{A}} w_{i}+\left\langle n^{l}\right\rangle_{B} \cdot \sum_{i=N_{A}+1}^{N_{C}} w_{i}}{\sum_{i=1}^{N_{C}} w_{i}}\right) \\
= & \mathcal{C}\left(\left\langle n^{k}\right\rangle_{A},\left\langle n^{l}\right\rangle_{A} \cdot \frac{\sum_{i=1}^{N_{A}} w_{i}}{\sum_{i=1}^{N_{C}} w_{i}}\right)+\underbrace{\mathcal{C}\left(\left\langle n^{k}\right\rangle_{A},\left\langle n^{l}\right\rangle_{B} \cdot \frac{\sum_{i=N_{A}+1}^{N_{C}} w_{i}}{\sum_{i=1}^{N_{C}} w_{i}}\right)}_{=0 \text { as } A \cap B=\varnothing} \\
= & \frac{\sum_{i=1}^{N_{A}} w_{i}}{\sum_{i=1}^{N_{C}} w_{i}} \mathcal{C}\left(\left\langle n^{k}\right\rangle_{A},\left\langle n^{l}\right\rangle_{A}\right) .
\end{aligned}
$$


We can write this correlation in terms of the underlying unweighted distributions as

$$
\begin{aligned}
\mathcal{C}\left(\left\langle n^{k}\right\rangle_{A},\left\langle n^{l}\right\rangle_{C}\right)= & \frac{\sum_{i=1}^{N_{A}} w_{i}^{2}}{\sum_{i=1}^{N_{C}} w_{i} \sum_{i=1}^{N_{A}} w_{i}} \cdot \mathcal{C}\left(n^{k}, n^{l}\right)_{A} \\
= & \frac{\sum_{i=1}^{N_{A}} w_{i}^{2}}{\sum_{i=1}^{N_{C}} w_{i} \sum_{i=1}^{N_{A}} w_{i}} \cdot\left\langle\left(n^{k}-\left\langle n^{k}\right\rangle\right)\left(n^{l}-\left\langle n^{l}\right\rangle\right)\right\rangle_{A} \\
= & \frac{\sum_{i=1}^{N_{A}} w_{i}^{2}}{\sum_{i=1}^{N_{C}} w_{i} \sum_{i=1}^{N_{A}} w_{i}} \cdot\left(\left\langle n^{k+l}\right\rangle_{A}-\left\langle n^{k}\right\rangle_{A}\left\langle n^{l}\right\rangle_{A}\right) .
\end{aligned}
$$

The correlation coefficients $\rho$ can be written, using their definition and eq. (6.48) for the variance, as

$$
\begin{aligned}
\rho\left(\left\langle n^{k}\right\rangle_{A},\left\langle n^{l}\right\rangle_{C}\right) & =\frac{\mathcal{C}\left(\left\langle n^{k}\right\rangle_{A},\left\langle n^{l}\right\rangle_{C}\right)}{\sqrt{V\left(\left\langle n^{k}\right\rangle_{A}\right)} \sqrt{V\left(\left\langle n^{l}\right\rangle_{C}\right)}} \\
& =\frac{\sqrt{\sum_{i=1}^{N_{A}} w_{i}^{2}}}{\sqrt{\sum_{i=1}^{N_{C}} w_{i}^{2}}} \cdot \frac{\left\langle n^{k+l}\right\rangle_{A}-\left\langle n^{k}\right\rangle_{A}\left\langle n^{l}\right\rangle_{A}}{\sqrt{\left\langle n^{2 k}\right\rangle_{A}-\left\langle n^{k}\right\rangle_{A}^{2}} \sqrt{\left\langle n^{2 l}\right\rangle_{C}-\left\langle n^{l}\right\rangle_{C}^{2}}} .
\end{aligned}
$$

The resulting correlations will be discussed together with the results in section 6.10 .

\subsubsection{Calculation of Central Moments}

The central or so-called modified central moments, calculated w.r.t. to a central value of $1.35 \mathrm{GeV}^{2}$, proposed in [6] can be calculated from the measured moments applying the following non-linear transformations,

$$
\begin{aligned}
\left(\begin{array}{c}
\left\langle n_{X}^{2}\right\rangle \\
\left\langle n_{X}^{4}\right\rangle \\
\left\langle n_{X}^{6}\right\rangle
\end{array}\right) \stackrel{(1)}{\longrightarrow}\left(\begin{array}{c}
\left\langle n_{X}^{2}\right\rangle \\
\left\langle\left(n_{X}^{2}-\left\langle n_{X}^{2}\right\rangle\right)^{2}\right\rangle \\
\left\langle\left(n_{X}^{2}-\left\langle n_{X}^{2}\right\rangle\right)^{3}\right\rangle
\end{array}\right) \\
\text { and } \stackrel{(2)}{\longrightarrow}\left(\begin{array}{c}
\left\langle n_{X}^{2}\right\rangle \\
\left\langle\left(n_{X}^{2}-1.35 \mathrm{GeV}^{2}\right)^{2}\right\rangle \\
\left\langle\left(n_{X}^{2}-1.35 \mathrm{GeV}^{2}\right)^{3}\right\rangle
\end{array}\right) .
\end{aligned}
$$

The two sets of central moments have similar values and show the same dependence on the $p_{\ell, \mathrm{BRF}}$ cut.

The covariance matrix $\mathcal{C}^{\prime}$ for the new vector of central moments can be calculated in first order using the Jacobian matrix $\mathcal{J}$ for the transformation and the initial covariance matrix $\mathcal{C}$

$$
\mathcal{C}_{(i)}^{\prime}=\mathcal{J}_{(i)} \mathcal{C} \mathcal{J}_{(i)}^{T}
$$


This approximation yields for the variance of the central moments the same results as Gaussian error propagation. The Jacobians are $36 \times 36$ matrices given by

$$
\begin{aligned}
& \mathcal{J}_{(1)}=\left(\begin{array}{rrr}
\mathbb{1}_{(12)} & 0_{(12)} & 0_{(12)} \\
{\left[-2\left\langle n_{X}^{2}\right\rangle\right] \mathbb{1}_{(12)}} & \mathbb{1}_{(12)} & 0_{(12)} \\
{\left[-3\left\langle n_{X}^{4}\right\rangle+6\left\langle n_{X}^{2}\right\rangle^{2}\right] \mathbb{1}_{(12)}} & {\left[-3\left\langle n_{X}^{2}\right\rangle\right] \mathbb{1}_{(12)}} & \mathbb{1}_{(12)}
\end{array}\right) \text { and } \\
& \mathcal{J}_{(2)}=\left(\begin{array}{rrr}
\mathbb{1}_{(12)} & 0_{(12)} & 0_{(12)} \\
{[-2 k] \mathbb{1}_{(12)}} & \mathbb{1}_{(12)} & 0_{(12)} \\
{\left[-3 k^{2}\right] \mathbb{1}_{(12)}} & {[-3 k] \mathbb{1}_{(12)}} & \mathbb{1}_{(12)}
\end{array}\right) \quad \text { with } k=1.35 \mathrm{GeV}^{2}
\end{aligned}
$$

with $\mathbb{1}_{(12)}$ the $12 \times 12$ unity matrix and $0_{(12)}$ a $12 \times 12$ matrix containing zeros. Each $12 \times 12$ block represents one type of moments for different cuts on the lepton momentum.

\subsection{Investigations and Optimizations of the Moment Extraction}

Several steps during the presented analysis have been investigated and optimized regarding the performance of the moment extraction method. All distributions relevant for selection criteria or binning of calibration curves are compared between data and simulation, described in section 6.9.1. The investigation of two of the applied cuts is described in section 6.9.2 and 6.9.3. The whole extraction procedure is verified on MC simulations, which is presented in section 6.9.4.

\subsubsection{Comparison of Simulated with Data Distributions}

In the analysis several kinematic variables or reconstructed quantities of the event are important as they are either used for analysis cuts or used for choosing a calibration curve. These are especially $E_{\text {miss }}-c\left|\vec{p}_{\text {miss }}\right|$ and the charged and neutral multiplicity of the $X_{c}$ system. The measured distribution of $n_{X}^{2}$ is compared between data and simulation, too. In this section, comparison plots between the relevant distributions on data and generic $B \bar{B}$ $\mathrm{MC}$ are discussed. In all distributions shown, combinatorial background is subtracted. The MC shapes are normalized to the same integral as the data distributions to identify differences in the shapes. Normalizing to the same number of correctly reconstructed $B_{\text {reco }}$ mesons yields similar results. Whenever discrepancies are visible in the comparisons, they are investigated as sources of systematic uncertainties, which are discussed in chapter 7 .

The comparison of the shapes in $E_{\text {miss }}-c\left|\vec{p}_{\text {miss }}\right|$ shows a good agreement between data and simulation in the positive part, cf. figure 6.24. In the negative part, we observe discrepancies. In the steep negative edge more events are reconstructed in MC, while in the far negative tail at $E_{\text {miss }}-c\left|\vec{p}_{\text {miss }}\right| \sim-1 \mathrm{GeV}$ the data exceeds the simulation by about $50 \%$. Negative values of $E_{\text {miss }}-c\left|\vec{p}_{\text {miss }}\right|$ are caused by additionally measured energy, e. g. caused by hadronic split-offs in the calorimeter or beam-generated background photons. As a consequence of the observed differences, we apply the narrow cut on $E_{\text {miss }}-c\left|\vec{p}_{\text {miss }}\right|$ between -0.2 and $0.3 \mathrm{GeV}$. Furthermore, we evaluate the influence of this cut in $E_{\text {miss }}-c\left|\vec{p}_{\text {miss }}\right|$ and the observed differences as systematic effects as described in section 7.1.10.

The simulation of the charged multiplicity of the $X_{c}$ system shows a good agreement with the distribution measured in data as shown in figure 6.25. Small differences are visible for $N_{\text {trk }, X}=2$ and at high multiplicities for $p_{\ell, \mathrm{BRF}} \geq 1.7 \mathrm{GeV} / c$. 

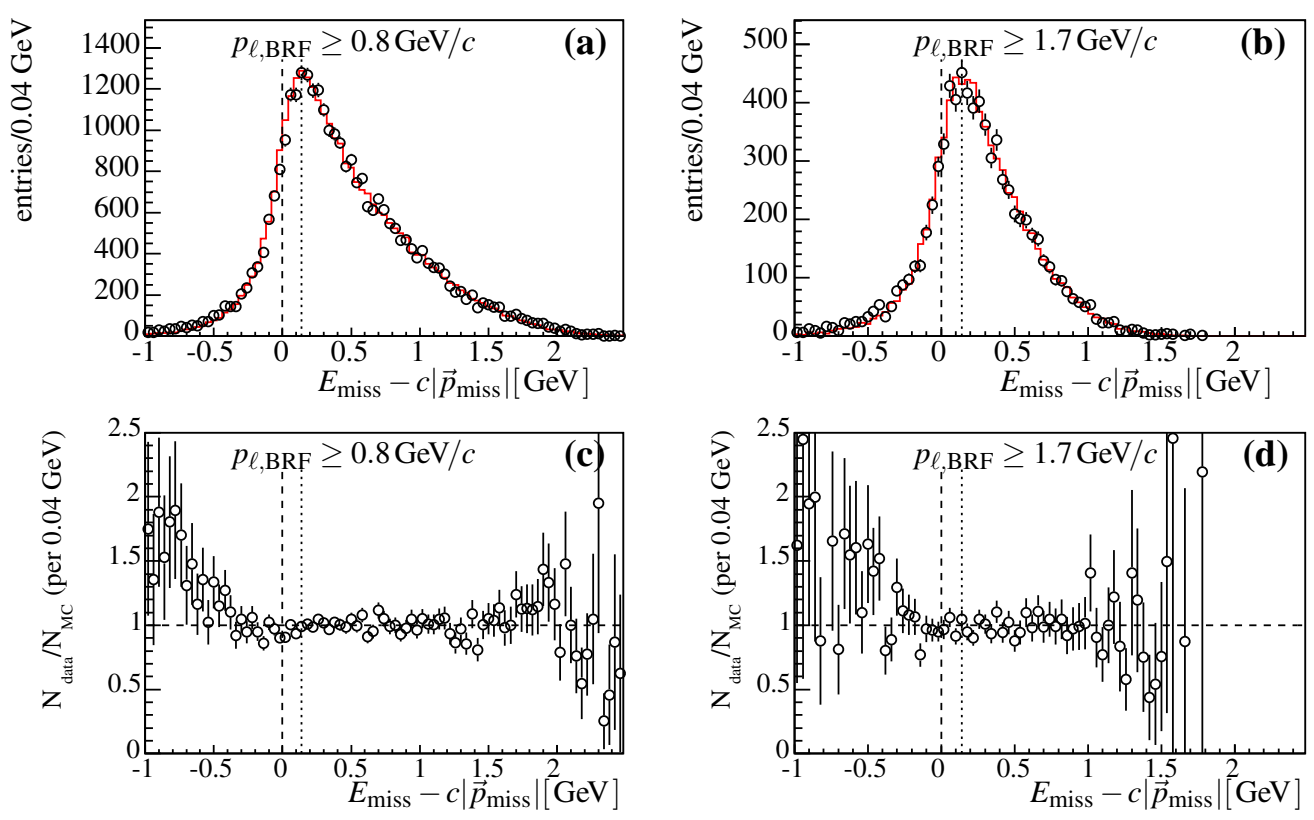

Figure 6.24: Comparison of data (o) and MC distributions (red histogram) for $E_{\text {miss }}-$ $c\left|\vec{p}_{\text {miss }}\right|$ for two different cuts on the lepton momentum, $p_{\ell, \mathrm{BRF}} \geq 0.8 \mathrm{GeV} / c$ (a) and $p_{\ell, \mathrm{BRF}} \geq 1.7 \mathrm{GeV} / c$ (b). The distributions are shown for the whole selected datasets and are normalized to the same integral to allow a shape comparison. The ratio of the two distributions is shown in plots (c) and (d). A dashed (dotted) line is drawn at $E_{\text {miss }}-c\left|\vec{p}_{\text {miss }}\right|=0$ (at the peak position $E_{\text {miss }}-c\left|\vec{p}_{\text {miss }}\right| \sim 0.12 \mathrm{GeV}$ ).

In contrast, the neutral multiplicity shows a shift of the MC distribution towards higher multiplicities w. r. t. to the data, see figure 6.26. Further investigations show that this shift is firstly only caused by the SP6/Run 4 portion of the data, and secondly is only visible for low energetic neutral candidates. This is illustrated in figure 6.27, where the multiplicity per event is plotted in bins of the neutrals' energy. The discrepancy vanishes for $E_{\text {neutral }}>0.1 \mathrm{GeV}$, as shown in figure $6.27(\mathrm{e}-\mathrm{h})$. It is known that this difference is caused by an overestimated admixture of beam-generated background photons to the MC sample simulated for the Run 4 dataset.

The $n_{X}^{2}$ distribution measured in data is generally well described by our MC simulation, cf. figure 6.28. Large values of $n_{X}^{2}$ above $4 \mathrm{GeV}^{2}$ seem to be more frequently reconstructed in MC than in data. These high values of $n_{X}^{2}$ are obtained from decays $B \rightarrow D^{* *} \ell v$ and $B \rightarrow D^{(*)} \pi \ell v$, which are connected with large uncertainties of their branching ratios. These branching ratios are covered as a source of systematic uncertainty by degrading or enhancing the corresponding contributions in our MC simulation as described in section 7.1.8. 

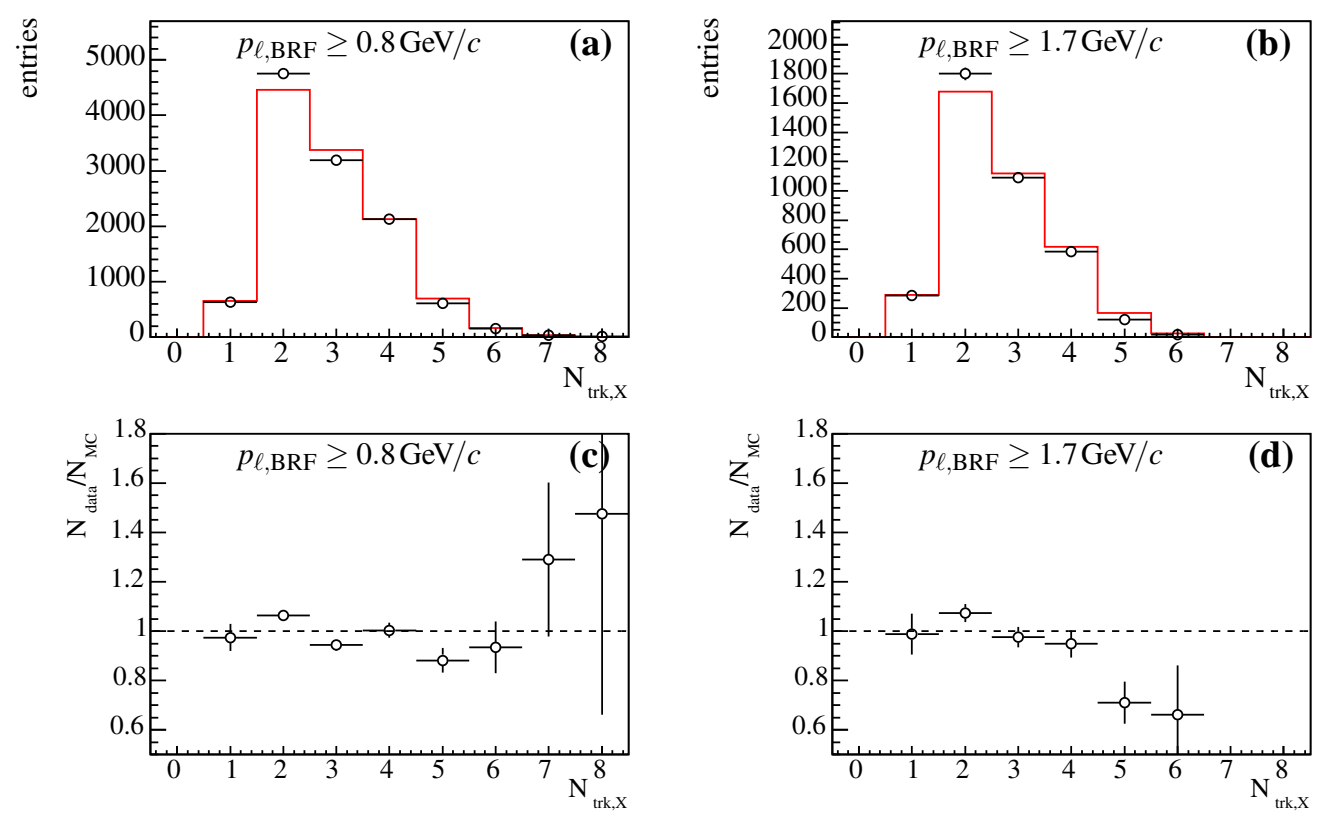

Figure 6.25: Comparison of data (o) and $\mathrm{MC}$ distributions (red histogram) for the charged multiplicity of the $X_{c}$ system $N_{\text {trk }, X}$ for $p_{\ell, \mathrm{BRF}} \geq 0.8 \mathrm{GeV} / c$ (a) and $p_{\ell, \mathrm{BRF}} \geq 1.7 \mathrm{GeV} / c$ (b). The distributions are normalized to the same integral to allow a shape comparison. The ratio of the two distributions is shown in plots (c) and (d).
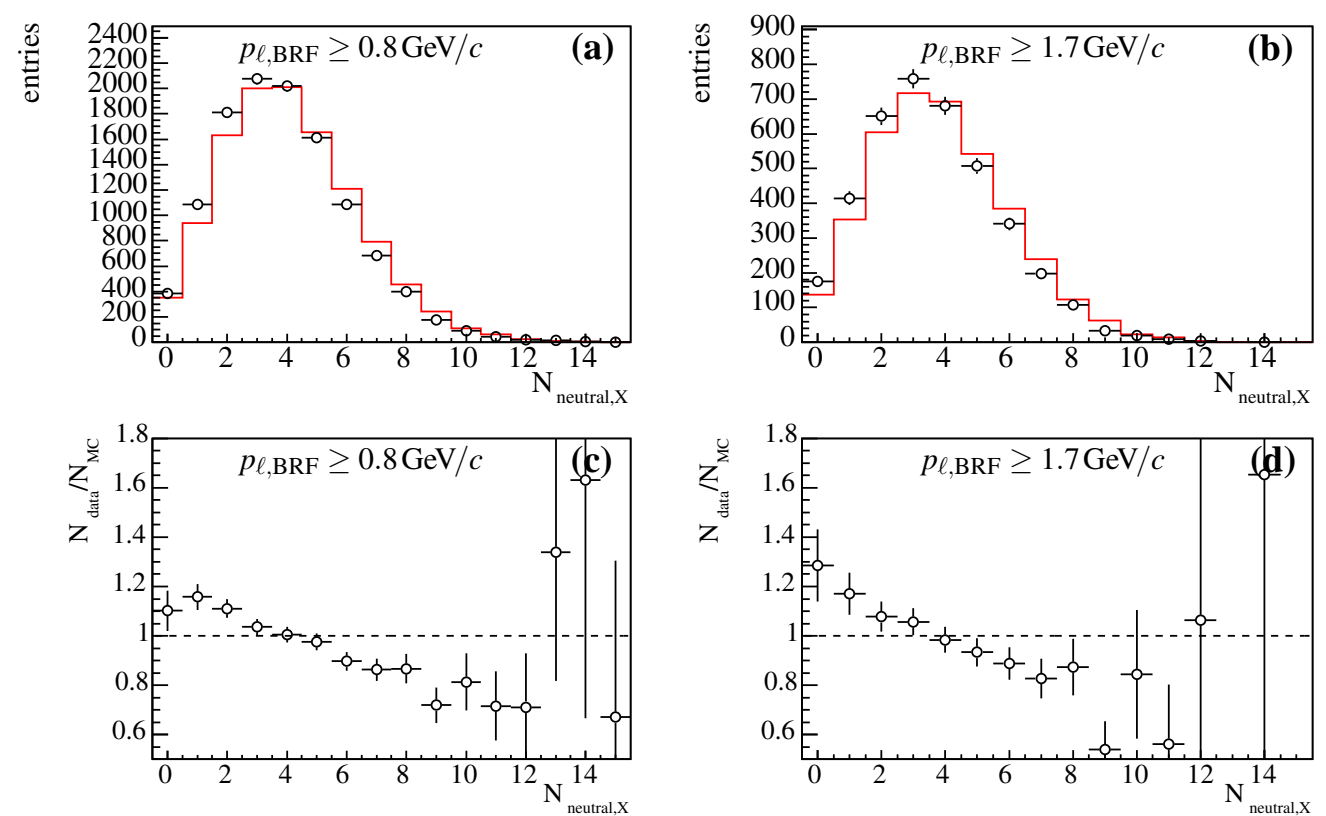

Figure 6.26: Comparison of data ( $\circ$ ) and $M C$ distributions (red histogram) for the neutral multiplicity of the $X_{c}$ system $N_{\text {neutral }, X}$ for $p_{\ell, \mathrm{BRF}} \geq 0.8 \mathrm{GeV} / c$ (a) and $p_{\ell, \mathrm{BRF}} \geq 1.7 \mathrm{GeV} / c$ (b). The distributions are normalized to the same integral to allow a shape comparison. The ratio of the two distributions is shown in plots (c) and (d). 

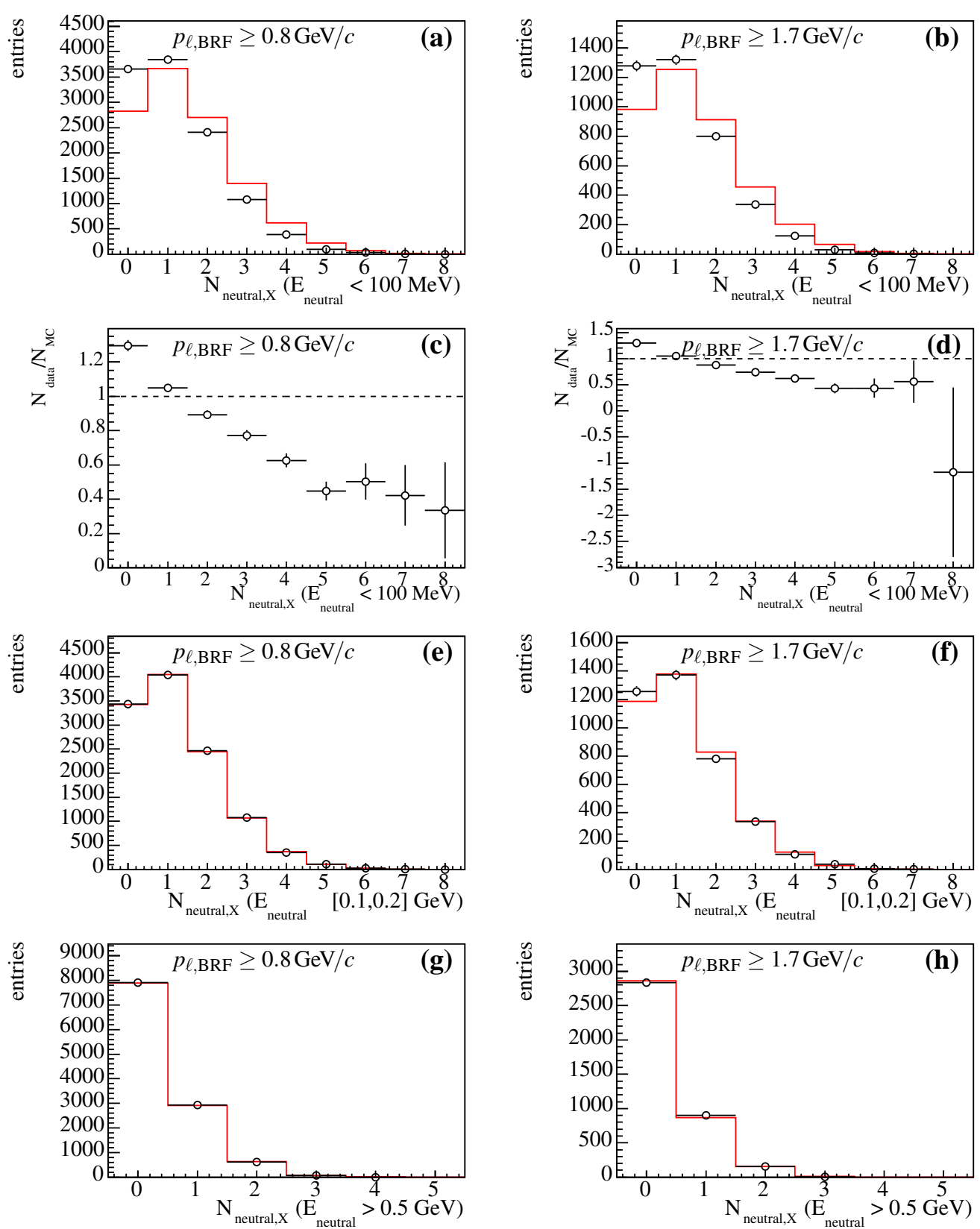

Figure 6.27: Comparison of data (o) and MC distributions (red histogram) for the neutral multiplicity of the $X_{c}$ system with $E_{\text {neutral }}<100 \mathrm{MeV}$ for $p_{\ell, \mathrm{BRF}} \geq 0.8 \mathrm{GeV} / c$ (a) and $p_{\ell, \mathrm{BRF}} \geq 1.7 \mathrm{GeV} / c$ (b) (ratios $N_{\text {data }} / N_{\mathrm{MC}}$ in plots (c) and (d)). The distributions are normalized to the same integral to allow a shape comparison. Two higher bins in $E_{\text {neutral }}$ are shown in plots (e)-(h), $E_{\text {neutral }} \in[0.1,0.2] \mathrm{GeV}$ in plots (e) and (f), and $E_{\text {neutral }}>$ $0.5 \mathrm{GeV}$ in plots $(\mathrm{g})$ and $(\mathrm{h})$. 

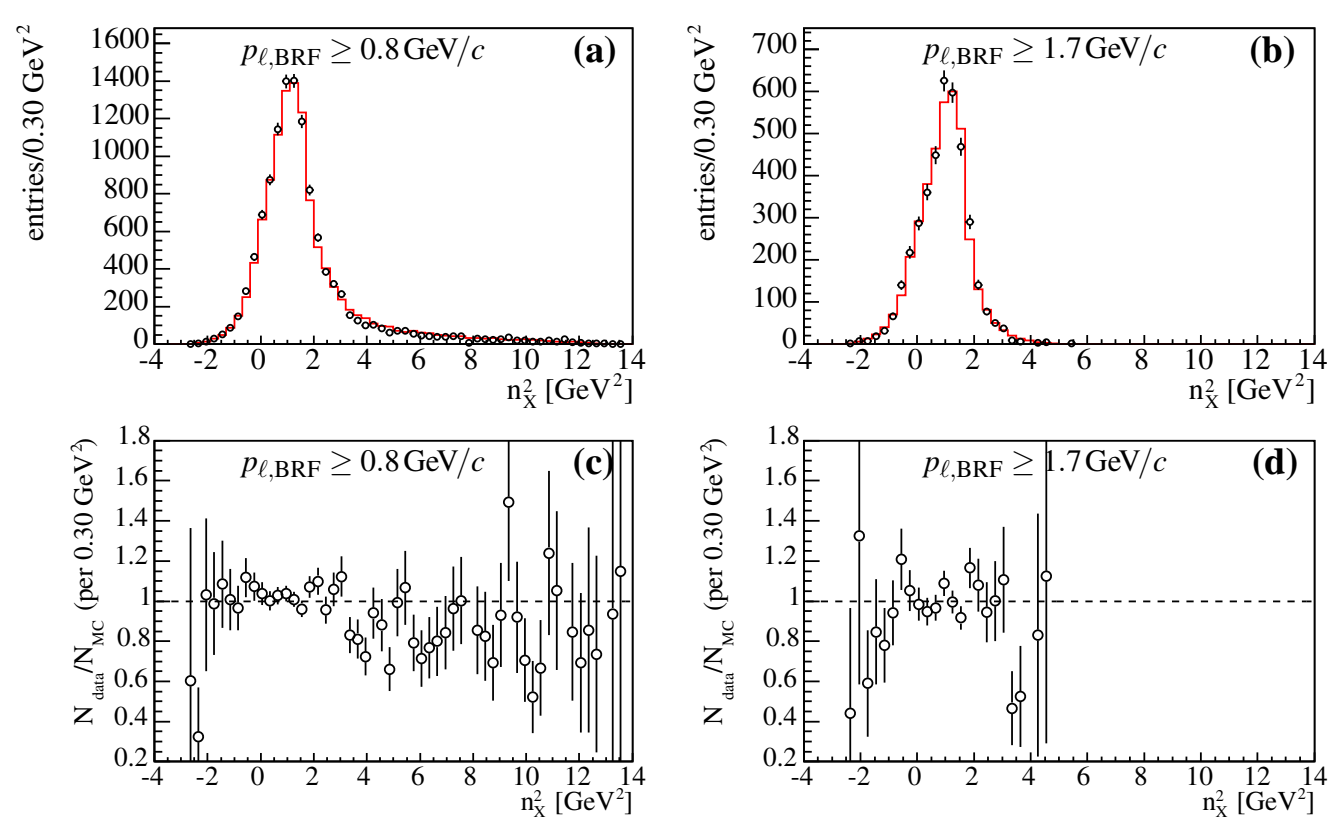

Figure 6.28: Comparison of data ( $\circ$ ) and $\mathrm{MC}$ distributions (red histogram) for $n_{X}^{2}$ for $p_{\ell, \mathrm{BRF}} \geq 0.8 \mathrm{GeV} / c$ (a) and $p_{\ell, \mathrm{BRF}} \geq 1.7 \mathrm{GeV} / c$ (b). The distributions are normalized to the same integral to allow a shape comparison. The ratio of the two distributions is shown in plots (c) and (d).

\subsubsection{Optimization of the Total Charge Requirement}

Previous measurements of hadronic moments allowed a maximal charge imbalance of one in the event. In this analysis this cut has been investigated. Intuitively, the restriction to $Q_{\text {tot,event }}=0$ promises a better resolution. On the other hand, this tighter requirement would reduce the selected signal decays in the data sample by $31 \%$ as shown in figure 6.29 , thereby increasing the statistical uncertainty.

The cut has been chosen according to the performance of the calibration procedure. The measured bias of the moments before the calibration does not change significantly when the cut is tightened. However, the remaining bias correction after the calibration shows a difference, although this is not consistent for the moments $\left\langle n_{X}^{2}\right\rangle,\left\langle n_{X}^{4}\right\rangle$, and $\left\langle n_{X}^{6}\right\rangle$. While the calibrated moments $\left\langle n_{X}^{2}\right\rangle$ show a smaller bias without the tight requirement on $Q_{\text {tot,event }}$, the higher order moments benefit from the tighter cut. We decide to keep the allowed maximal charge imbalance of one, $\left|Q_{\text {tot,event }}\right| \leq 1$.

\subsubsection{Investigation of the Selected Region in $\mathbf{E}_{\text {miss }}-\mathbf{c} \overrightarrow{\mathbf{p}}_{\text {miss }}$}

Closely related to the previous discussion of the optimization of the cut on $Q_{\text {totevent }}$ is the choice of the selected region in $E_{\text {miss }}-c\left|\vec{p}_{\text {miss }}\right|$. Figure 6.30 shows that mean and RMS of the distribution $n_{X \text {,true }}^{2}-n_{X \text {,reco }}^{2}$ after the kinematic fit strongly depend on the value of $E_{\text {miss }}-c\left|\vec{p}_{\text {miss }}\right|$. Reasonable values of the mean ( + or $-0.2 \mathrm{GeV}^{2}$ ) are only achieved in the region $-0.2<E_{\text {miss }}-c\left|\vec{p}_{\text {miss }}\right|<0.7 \mathrm{GeV}$. However, in this region, the RMS of 


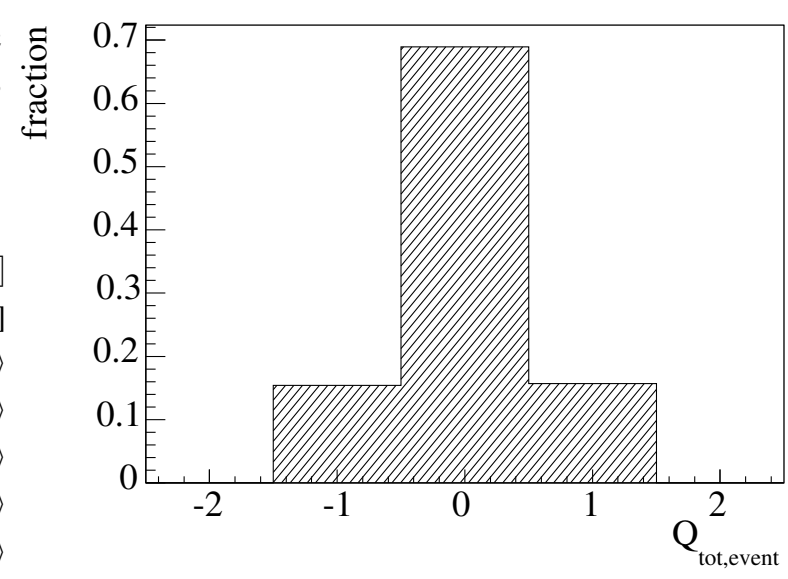

Figure 6.29: Distribution of $Q_{\text {totevent }}$ in the selected event sample in data. Background is subtracted using event weights. The histogram is normalized to unity. About $69 \%$ of the events fulfill $Q_{\text {tot,event }}=0$.

$n_{X, \text { true }}^{2}-n_{X, \text { reco }}^{2}$ goes up to about $1.6 \mathrm{GeV}^{2}$ at $E_{\text {miss }}-c\left|\vec{p}_{\text {miss }}\right|=0.7 \mathrm{GeV}$, while the minimal RMS at $E_{\text {miss }}-c\left|\vec{p}_{\text {miss }}\right| \sim 0 \mathrm{GeV}$ is $1.1 \mathrm{GeV}^{2}$. In the negative part of $E_{\text {miss }}-c\left|\vec{p}_{\text {miss }}\right|$, the RMS only increases by about $0.2 \mathrm{GeV}$. Thus, restricting the positive region to comparable values of RMS, we pre-select a region in $E_{\text {miss }}-c\left|\vec{p}_{\text {miss }}\right|$ between -0.2 and $0.4 \mathrm{GeV}$. This region is further investigated to optimize the moment extraction procedure w. r.t. the bias remaining after calibration.

We find that the influence of the selected region in $E_{\text {miss }}-c\left|\vec{p}_{\text {miss }}\right|$ has only little influence on the bias remaining after calibration. Overall, we achieve the best results for the selected region $-0.2<E_{\text {miss }}-c\left|\vec{p}_{\text {miss }}\right|<0.3 \mathrm{GeV}$. Furthermore, for large values of $E_{\text {miss }}-c\left|\vec{p}_{\text {miss }}\right|$, the calibration curves flatten, which makes it rather problematic to invert them for the calibration process. Therefore, we keep the region $-0.2<E_{\text {miss }}-c\left|\vec{p}_{\text {miss }}\right|<0.3 \mathrm{GeV}$ for the analysis.

\subsubsection{Verification of the Calibration Procedure}

To check whether the extraction formalism, that is calibration and bias correction, works correctly, several tests are performed. They use either MC events which have not entered into the extraction of the calibration curves and bias correction factors or subsets of signal events containing only one exclusive decay channel.

Test on Divided Generic Monte Carlo Sample A very realistic test with respect to data calibration uses the generic MC dataset. It is split into two parts according to even and odd event identification numbers (event IDs). The moments are determined on one subset containing half of the events while the second half of events is used for the subtraction of residual background and the determination of bias correction factors. Two checks are performed, one with the default subdivision and another with interchanged samples. In more detail, the following procedure is performed.

- The calibration curves are determined on full Cocktail MC dataset.

- Even (odd) event IDs are used for two purposes: 


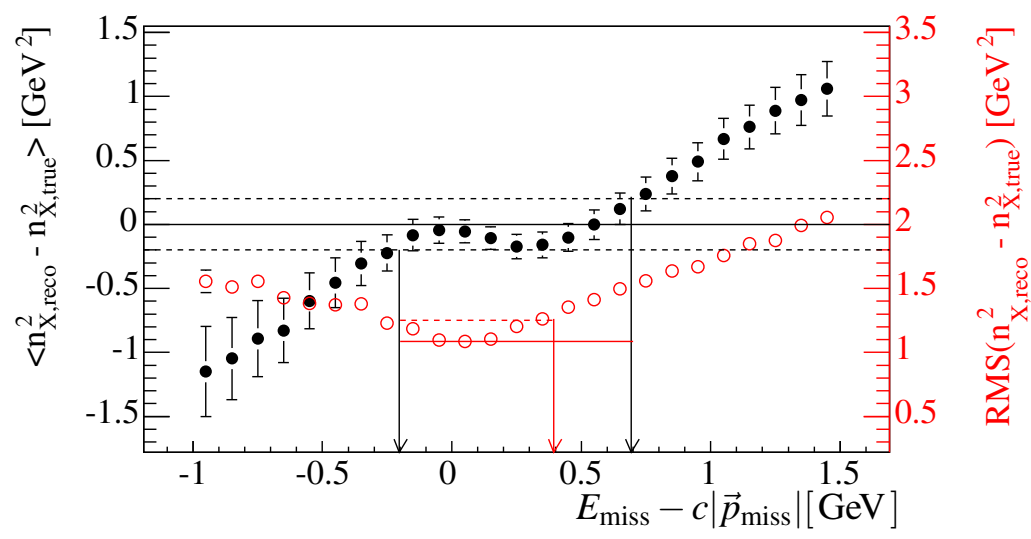

Figure 6.30: Mean (•, left axis) and RMS (०, right axis) of the distribution $n_{X, \text { true }}^{2}-n_{X, \text { reco }}^{2}$ after the kinematic fit in bins of $100 \mathrm{MeV}$ in $E_{\text {miss }}-c\left|\vec{p}_{\text {miss }}\right|$. Selecting a region with $\left|\left\langle n_{X \text {,true }}^{2}-n_{X \text {,reco }}^{2}\right\rangle\right|<0.2 \mathrm{GeV}^{2}$ (dashed black lines and $\downarrow$ ) and comparable values of RMS (dashed red line) w. r. t. the minimal RMS (solid red line) leads to a pre-selected region in $E_{\text {miss }}-c\left|\vec{p}_{\text {miss }}\right|$ of $-0.2<E_{\text {miss }}-c\left|\vec{p}_{\text {miss }}\right|<0.4 \mathrm{GeV}(\downarrow)$.

- The background events are used for subtraction of the residual background peaking in $m_{\mathrm{ES}}$.

- The signal component is used for the derivation of the final bias correction. Therefore only this signal component is calibrated beforehand. The correction factors are determined according to equation 6.47 .

- Odd (even) event IDs are treated like data in the final analysis. They are calibrated and the final bias correction is applied. The shape of the combinatorial background is measured on this data sample.

This study allows a check of the complete extraction formalism. If the true moments are reobtainted after the extraction, the procedure is able to yield reliable results. Figure 6.31 shows the results obtained. The datapoint at $p_{\ell, \mathrm{BRF}} \geq 1.4 \mathrm{GeV} / c$ exceeds the deviation of one standard deviation. As all datapoints with a lower cut on $p_{\ell, \mathrm{BRF}}$ are correlated with this point, they deviate as well. The moments binned in the lepton momentum show the corresponding behavior. We consider this deviation of 2.2 standard deviations in one bin of $p_{\ell, \mathrm{BRF}}$ as a statistical deviation and do not add an additional uncertainty.

Test on Exclusive $\mathbf{b} \rightarrow \mathbf{c} \ell \boldsymbol{v}$ Modes Another test is performed by applying the calibration to measured $n_{X}^{2}$ distributions of individual semileptonic decays, $B \rightarrow D \ell v, B \rightarrow D^{*} \ell v$, four resonant decays $B \rightarrow D^{* *} \ell \nu$, and two non-resonant decays $B \rightarrow D^{(*)} \pi \ell v$. The calibration curves are derived on the Cocktail MC sample containing a mixture of all exclusive final states. Figure 6.32 shows the corrected moments $\left\langle n_{X}^{k}\right\rangle_{\text {calib }}$ as functions of the true moments for minimal lepton momenta $p_{\ell, \mathrm{BRF}} \geq 0.8 \mathrm{GeV} / c$ and $p_{\ell, \mathrm{BRF}} \geq 1.7 \mathrm{GeV} / c$. The calibration reproduces the true moments over the full $n_{X}^{2}$ range. 

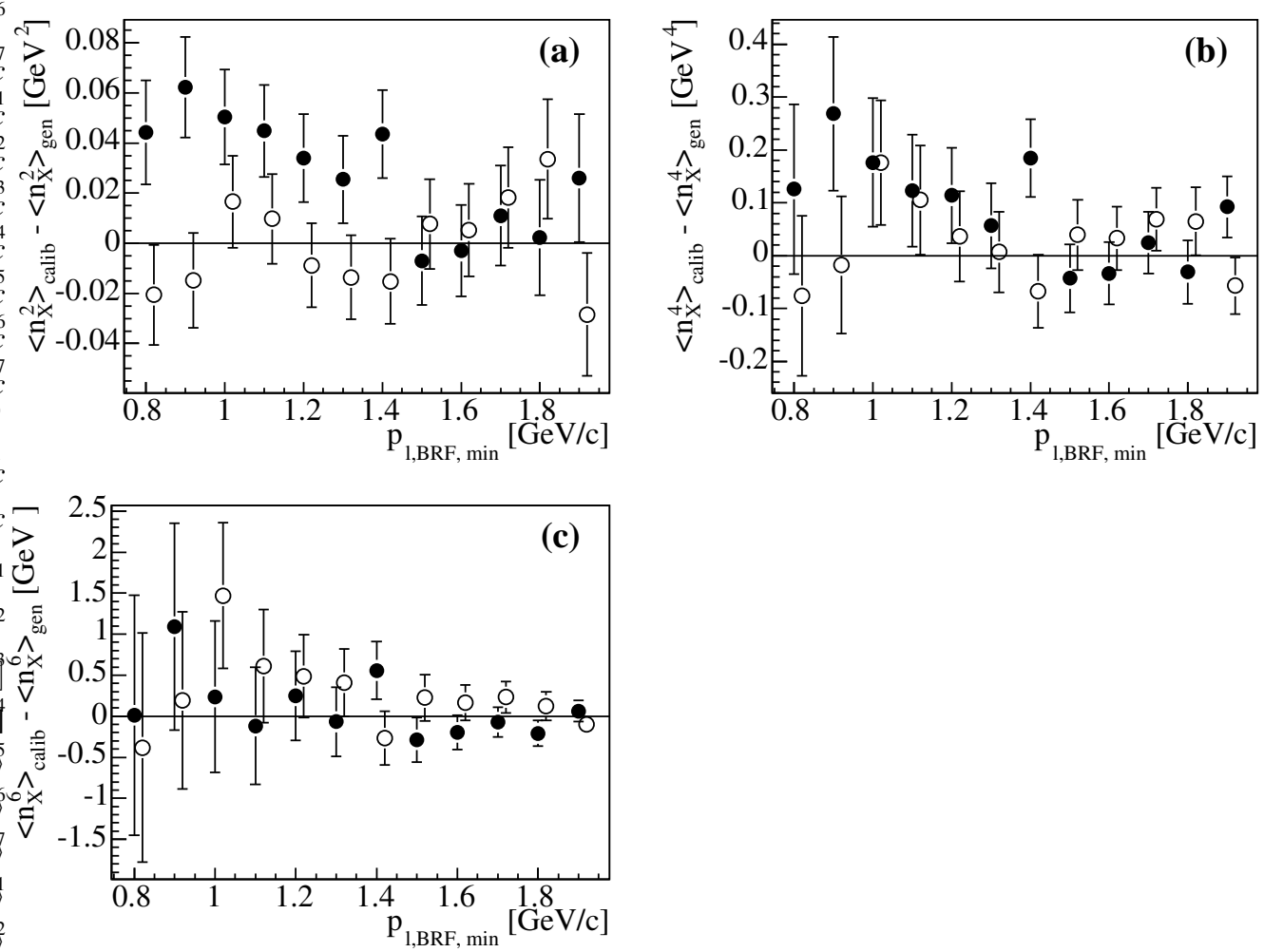

Figure 6.31: Verification of the analysis procedure on $\mathrm{MC}$ simulations for moments $\left\langle n_{X}^{2}\right\rangle$ (a), $\left\langle n_{X}^{4}\right\rangle$ (b), and $\left\langle n_{X}^{6}\right\rangle$ (c) as a function of the lower cut on $p_{\ell, \text { BRF. }}$. The moments are determined on a subset containing half of the events while the second half of events is used for the subtraction of residual background and the determination of bias correction factors. Two checks are performed, one with the default subdivision $(\bullet)$ and with interchanged samples (o).
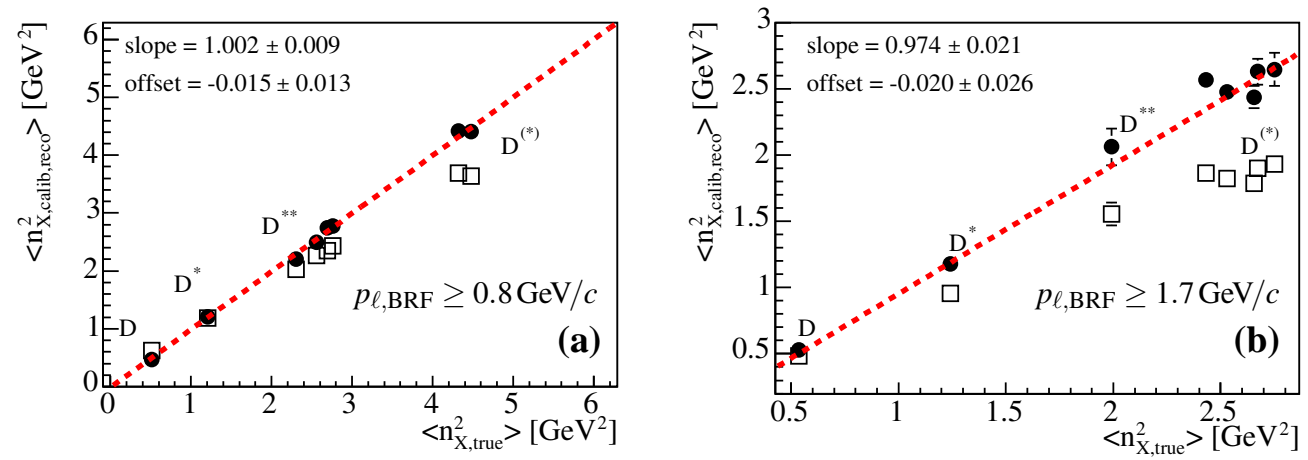

Figure 6.32: Result of the calibration verification procedure for different minimal lepton momenta $p_{\ell, \mathrm{BRF}} \geq 0.8 \mathrm{GeV} / c$ (a) and $p_{\ell, \mathrm{BRF}} \geq 1.7 \mathrm{GeV} / c$ (b). Moments $\left\langle n_{X}^{2}\right\rangle$ of exclusive modes on simulated events before calibration $(\square)$ and after calibration $(\bullet)$ plotted against the true moments for each mode. The dotted line shows the fit result to the calibrated moments, the resulting parameters are shown. 


\subsection{Results}

Figure 6.33 shows the extracted moments $\left\langle n_{X}^{k}\right\rangle$ with $k=2,4,6$ as functions of lower cuts on the lepton momentum $p_{\ell, \mathrm{BRF}}$. Also the central moments, determined as outlined in section 6.8.5, are shown. The moments are plotted together with their statistical and systematic uncertainties, which are discussed in chapter 7 . There, also summary tables with the numerical results are given. The numbers of selected events and of estimated background events for each cut on $p_{\ell, \mathrm{BRF}}$ are summarized in table 6.8.

As expected, a significant dependence on the minimal lepton momentum is observed manifesting in decreasing moments for higher cuts on $p_{\ell \text {.BRF }}$. It is the result of a reduced contribution of higher mass final states, which also have larger values of $n_{X}^{2}$, to the overall $n_{X}^{2}$ spectrum.

Statistical correlation coefficients for all measured moments are summarized in tables B.1 and B.2 in the appendix. We obtain correlations ranging between $4 \%$ and $99 \%$. They are illustrated as color map in figure 6.34 .

Table 6.8: Number of signal and background events for all cuts on $p_{\ell, \mathrm{BRF}}$. We quote the total number of measured events $N_{\text {total }}$, the numbers of background events $N_{\text {sideband }}$, $N_{\text {residual }}$, and their sum $N_{\text {bg,tot. }}$, and the corresponding number of signal events $N_{\text {signal }}$.

\begin{tabular}{lrrrrr}
\hline $\begin{array}{l}p_{\ell, \mathrm{BRF}} \\
{[\mathrm{GeV} / c]}\end{array}$ & $N_{\text {total }}$ & $N_{\text {sideband }}$ & $N_{\text {residual }}$ & $N_{\text {bg,tot. }}$ & $N_{\text {signal }}$ \\
\hline 0.8 & $13246 \pm 115$ & $1842 \pm 81$ & $1350 \pm 20$ & $3193 \pm 83$ & $10053 \pm 142$ \\
0.9 & $12452 \pm 112$ & $1626 \pm 79$ & $1167 \pm 18$ & $2792 \pm 81$ & $9660 \pm 138$ \\
1.0 & $11647 \pm 108$ & $1415 \pm 75$ & $994 \pm 18$ & $2409 \pm 77$ & $9238 \pm 133$ \\
1.1 & $10771 \pm 104$ & $1216 \pm 72$ & $877 \pm 16$ & $2093 \pm 74$ & $8678 \pm 127$ \\
1.2 & $9845 \pm 99$ & $1040 \pm 70$ & $753 \pm 15$ & $1793 \pm 71$ & $8052 \pm 122$ \\
1.3 & $8846 \pm 94$ & $959 \pm 72$ & $655 \pm 13$ & $1614 \pm 73$ & $7232 \pm 119$ \\
1.4 & $7789 \pm 88$ & $820 \pm 68$ & $568 \pm 13$ & $1388 \pm 69$ & $6401 \pm 112$ \\
1.5 & $6675 \pm 82$ & $719 \pm 67$ & $495 \pm 12$ & $1215 \pm 68$ & $5460 \pm 106$ \\
1.6 & $5566 \pm 75$ & $600 \pm 51$ & $429 \pm 11$ & $1029 \pm 52$ & $4537 \pm 91$ \\
1.7 & $4342 \pm 66$ & $442 \pm 44$ & $351 \pm 12$ & $793 \pm 45$ & $3549 \pm 80$ \\
1.8 & $3142 \pm 56$ & $309 \pm 38$ & $282 \pm 13$ & $591 \pm 41$ & $2551 \pm 69$ \\
1.9 & $2040 \pm 45$ & $187 \pm 22$ & $227 \pm 13$ & $414 \pm 25$ & $1626 \pm 52$ \\
\hline
\end{tabular}



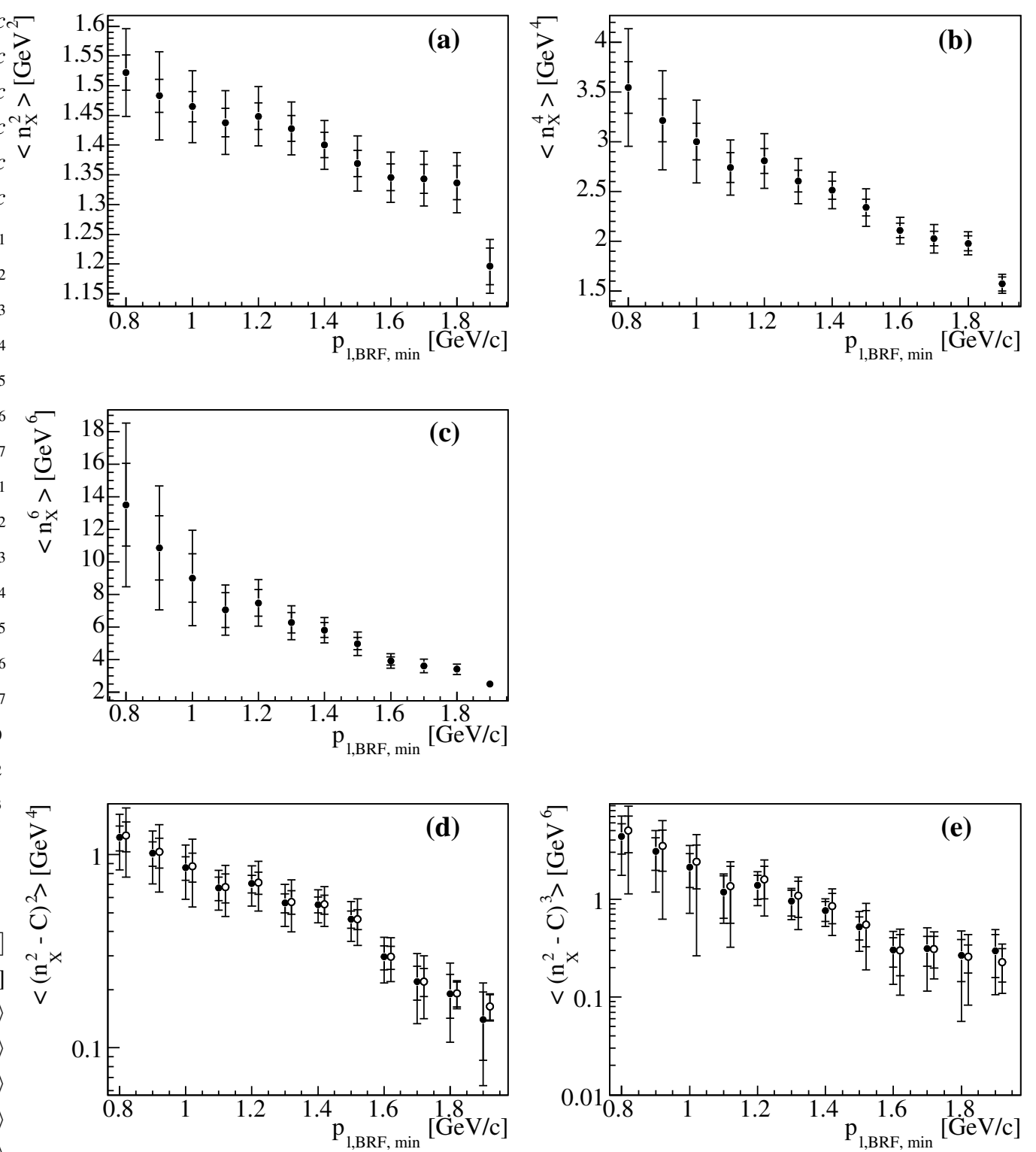

Figure 6.33: Measured moments $\left\langle n_{X}^{2}\right\rangle$ (a), $\left\langle n_{X}^{4}\right\rangle$ (b), $\left\langle n_{X}^{6}\right\rangle$ (c), and the central moments $\left\langle\left(n_{X}^{2}-C\right)^{2}\right\rangle$ with $C=\left\langle n_{X}^{2}\right\rangle(\bullet)$ and $C=1.35 \mathrm{GeV}^{2}(\mathrm{o})(\mathrm{d})$, and $\left\langle\left(n_{X}^{2}-C\right)^{3}\right\rangle$ with $C=$ $\left\langle n_{X}^{2}\right\rangle(\bullet)$ and $C=1.35 \mathrm{GeV}^{2}$ (०) (e) for different cuts on the lepton momentum $p_{\ell, \mathrm{BRF}}$. The error bars indicate the statistical and the total errors, respectively. Please note the logarithmic scale on the $y$-axis in plots (d) and (e). The moments are highly correlated. 

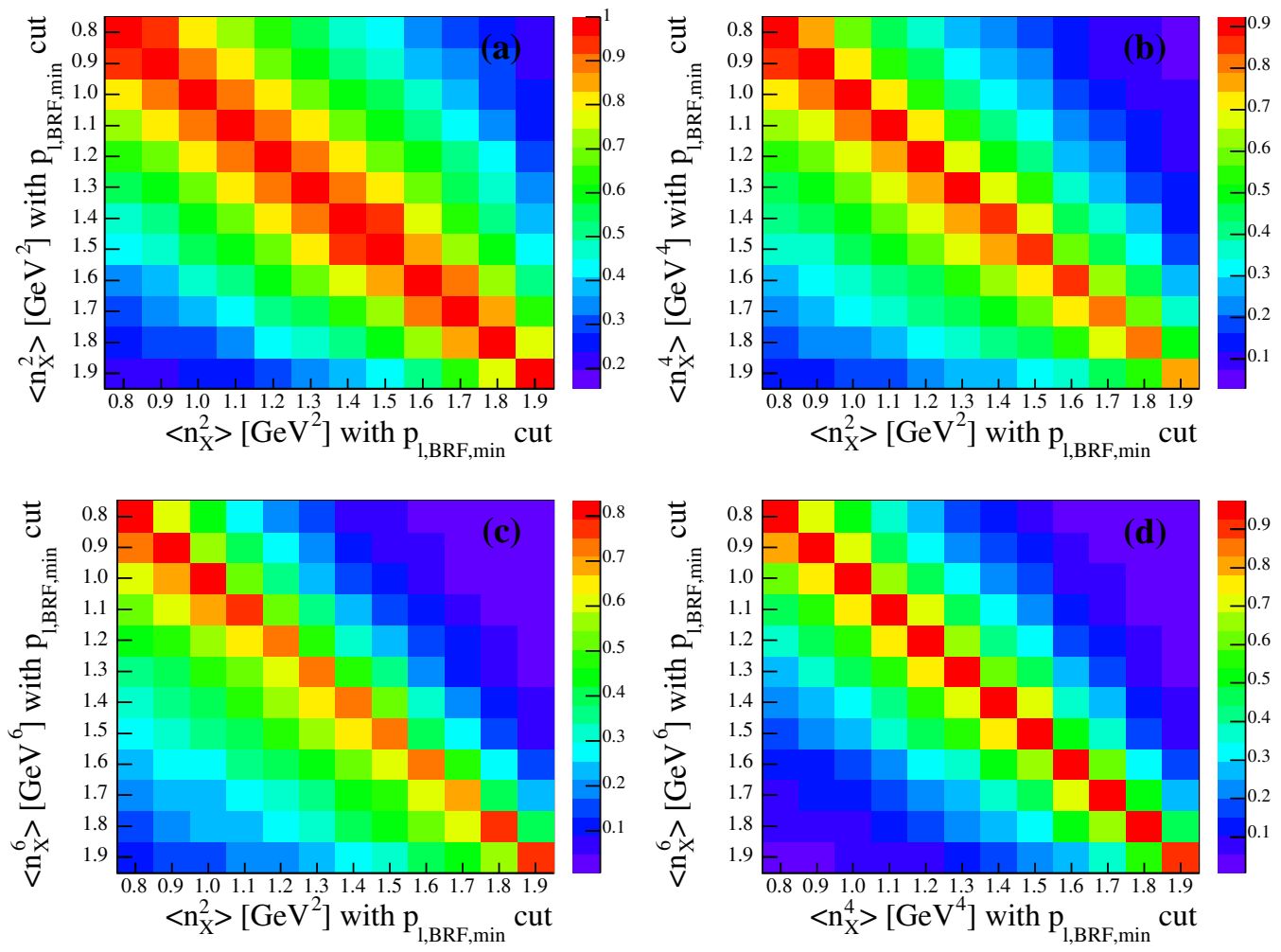

Figure 6.34: Color map of statistical correlation coefficients $\rho$ measured for the moments $\left\langle n_{X}^{k}\right\rangle$ with $k=2,4,6$ for the different cuts on the $p_{\ell, \mathrm{BRF}}$.

(a) $\rho\left(\left\langle n_{X}^{2}\right\rangle\left(p_{\ell, \mathrm{BRF}}\right),\left\langle n_{X}^{2}\right\rangle\left(p_{\ell, \mathrm{BRF}}\right)\right)$, (b) $\rho\left(\left\langle n_{X}^{2}\right\rangle\left(p_{\ell, \mathrm{BRF}}\right),\left\langle n_{X}^{4}\right\rangle\left(p_{\ell, \mathrm{BRF}}\right)\right)$,

(c) $\rho\left(\left\langle n_{X}^{2}\right\rangle\left(p_{\ell, \mathrm{BRF}}\right),\left\langle n_{X}^{6}\right\rangle\left(p_{\ell, \mathrm{BRF}}\right)\right),(\mathrm{d}) \rho\left(\left\langle n_{X}^{4}\right\rangle\left(p_{\ell, \mathrm{BRF}}\right),\left\langle n_{X}^{6}\right\rangle\left(p_{\ell, \mathrm{BRF}}\right)\right)$. 



\section{Evaluation of Systematic Uncertainties}

In this chapter several sources of systematic uncertainties are discussed. We derive estimates for the size of these uncertainties (see section 7.1), summarized and classified in section 7.2. Systematic cross checks are performed, testing the stability of our result on independent subsets of the data. They are described in section 7.3 .

\subsection{Estimates for Systematic Uncertainties}

We derive an estimate for the size of systematic uncertainties by deriving new moments on data with a changed setting in the extraction procedure regarding the source of the uncertainty under investigation, e.g. varied branching fractions in the MC simulation. The observed variation w. r.t. the nominal result is taken as estimate for the systematic uncertainty. The sources taken into account are discussed in the following.

\subsubsection{Statistical Uncertainty of the Combinatorial Background Subtraction}

Combinatorial background is subtracted from data itself using events outside the $m_{\mathrm{ES}}$ signal region for the determination of its $n_{X}^{2}$ distribution (see section 6.7.1). This method relies on fitting the $m_{\mathrm{ES}}$ distribution. Therefore, statistical uncertainties coming from these fits occur. The uncertainty on the $m_{\mathrm{ES}}$ fit parameters is directly propagated into the scaling factors $s$, which are used to scale the $n_{X}^{2}$ distribution of the combinatorial background. The size of the uncertainty on the moments is evaluated by varying these scaling factors within their errors randomly and thus extracting new background subtraction factors $w\left(n_{X}^{2}\right)$. Taking these modified factors, new sets of moments are calculated. This procedure is repeated 200 times, the resulting variation is fitted with a Gaussian and the standard deviation $\sigma$ of this Gaussian function is taken as error estimate. The error is about a factor 1.3 larger than the statistical uncertainty. For typical plots, see figure 7.1 (a)-(c).

\subsubsection{Statistical Uncertainty of the Simulated MC Sample}

Three additional uncertainties of statistical nature resulting from the limited MC dataset have been identified, which are counted as separate systematic uncertainty to distinguish them from the statistical uncertainty originating from the data sample. These are the statistical uncertainties of the calibration method, of the background weights for residual background, and of the bias correction factors, respectively.

1. The uncertainty of the moments arising from the statistical uncertainty of the calibration curves is evaluated by varying the parameters of the linear fits randomly within their fit errors taking their correlations into account, a method, we call Toy MC study. The modified set of calibration curves is used for extracting a new set of moments $\left\langle n_{X}^{k}\right\rangle$. This procedure is repeated 200 times yielding a distribution of 

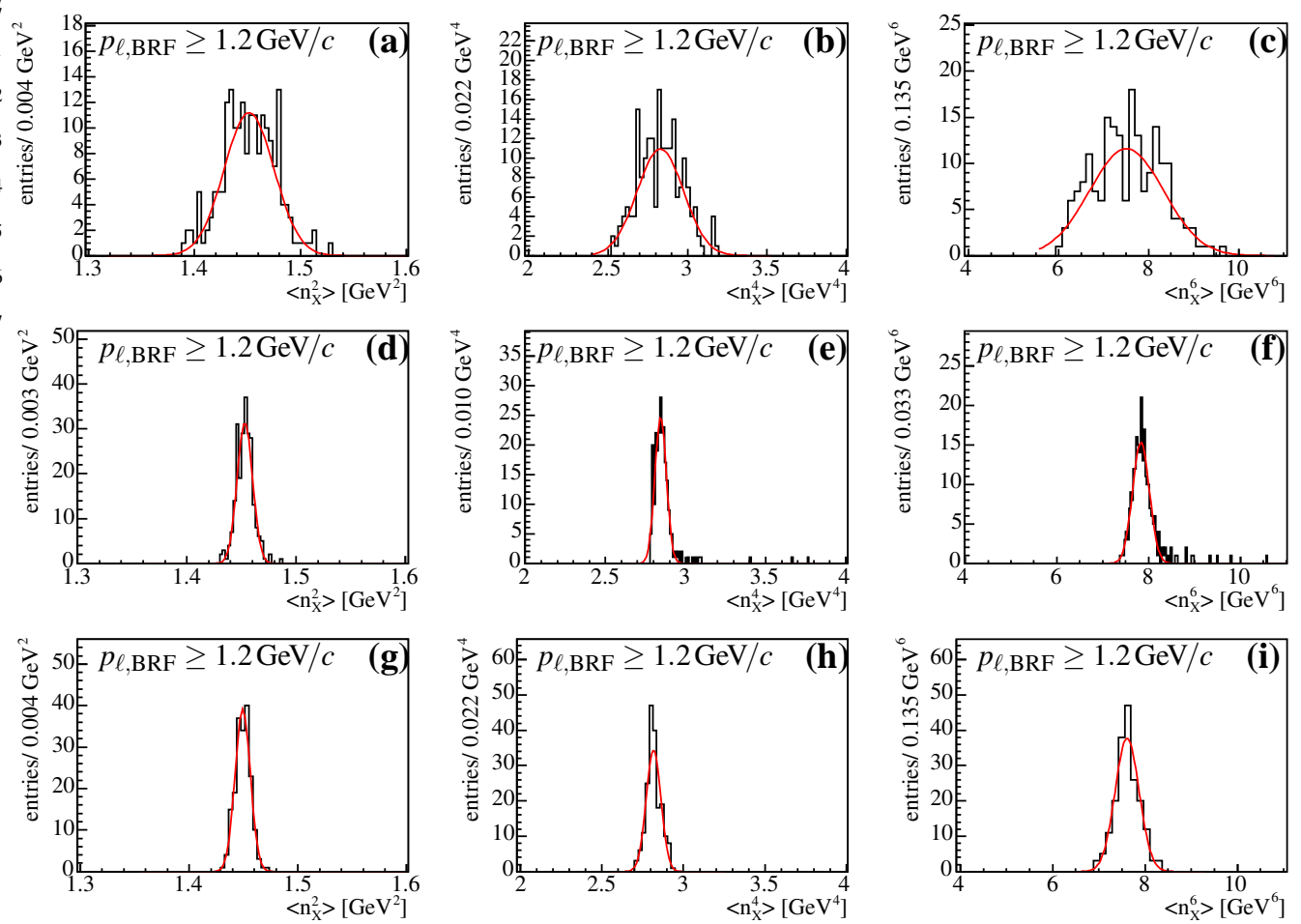

Figure 7.1: Distributions of measured moments when varying

- the background subtraction weights of the combinatorial background (a-c),

- the parameters of the linear fits of the calibration functions (d-f),

- and the background subtraction weights of the residual background (g-i)

randomly within their fit errors. (a/d/g) show the distributions for $\left\langle n_{X}^{2}\right\rangle$, (b/e/h) for $\left\langle n_{X}^{4}\right\rangle$, and (c/e/i) for $\left\langle n_{X}^{6}\right\rangle$, all measured for $p_{\ell, \mathrm{BRF}} \geq 1.2 \mathrm{GeV} / c$. The distributions are fitted with a Gaussian (red line), the standard deviation of which is taken as estimate for the systematic uncertainty. The error resulting from the background subtraction weights on data are a factor 5 larger than the one resulting from the calibration functions. 
moments. The standard deviation of a fit with a Gaussian to this distribution is taken as uncertainty. Some typical distributions are shown in figure 7.1(d)-(f). The error is smaller than half the statistical uncertainty.

2. Residual background peaking in $m_{\mathrm{ES}}$ is subtracted using $\mathrm{MC}$ simulations as described in section 6.7.2. The $m_{\mathrm{ES}}$ distribution of these background events is fitted and the combinatorial background is subtracted to get only the contribution peaking in $m_{\mathrm{ES}}$. As this procedure relies on fitting the $m_{\mathrm{ES}}$ distribution, statistical uncertainties coming from these fits occur. The uncertainty on the $m_{\mathrm{ES}}$ fit parameters is directly propagated into the scaling factors $s$, which are used to scale the $n_{X}^{2}$ distribution of the background. The size of the uncertainty is evaluated in the same way as for the combinatorial background, described in subsection 7.1.1. The error is about a factor 1.5 to 2 larger than the uncertainty arising from the calibration curves. It is a factor of 3 smaller than the uncertainty arising from the combinatorial background subtraction. For typical plots, see figure 7.1 (g)-(i).

3. The statistical error arising from the bias correction factor is due to uncertainties of the extracted moments in MC simulations. Here, we also evaluate the statistical uncertainty arising from the combinatorial background subtraction on the bias correction factor $C$ using Toy MC studies (same procedure as in 2.). This error is negligible for all cuts on the lepton momentum. The total statistical uncertainty of the bias correction factor is propagated into the statistical error of the moment by Gaussian error propagation,

$$
\begin{aligned}
\left\langle n_{X}^{k}\right\rangle_{\text {biascorr. }} & =C \cdot\left\langle n_{X}^{k}\right\rangle \\
\sigma\left(\left\langle n_{X}^{k}\right\rangle_{\text {biascorr. }}\right) & =\sqrt{C^{2} \cdot \sigma_{\left\langle n_{X}^{k}\right\rangle}^{2}+\left\langle n_{X}^{k}\right\rangle^{2} \cdot \sigma_{C}^{2}} .
\end{aligned}
$$

This error is small compared to the other two contributions discussed above. Thus, it is not listed separately but added directly to the statistical uncertainty of the moments.

The other two uncertainties are summarized in tables 7.2 to 7.4 together with the central values of the moments.

\subsubsection{Branching Fractions of Background Decays}

The shape and amount of the remaining background after the $m_{\mathrm{ES}}$ sideband subtraction is taken from MC simulations. The branching fractions of the simulated background decay channels are rescaled to recent experimental measurements, as discussed in section 6.7.2. The latest experimental uncertainties $\sigma_{\mathcal{B}}$ for these branching fractions are propagated into the result for the moments by rescaling each mode with the scaling factors $s=s_{\text {nominal }} \pm \sigma_{\mathcal{B}}$ (according to table 6.7). The mean observed variation of the moments for the variation of each background channel is listed in tables 7.2 to 7.4 .

It turns out that, despite their small branching fractions, the $b \rightarrow u \ell v$ decays with their comparably large uncertainty of $15 \%$ contribute dominantly to the total error from background decays. All exclusive $b \rightarrow u \ell v$ decays have been treated in common. As, in total, 

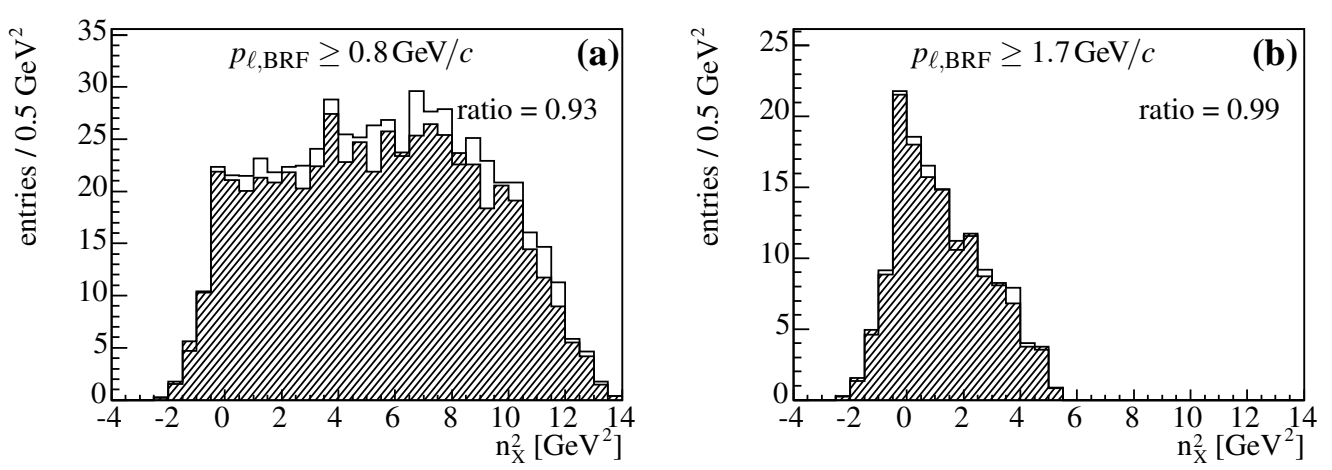

Figure 7.2: Background component due to fake leptons for standard PID tweaking ( $\square$ ) and for changed tweaking probability (hatched histogram). The ratio given is the ratio of the integrals of the two histograms. The two shapes are compatible within uncertainties.

the uncertainty arising from backgrounds is small compared to others, a variation of the single exclusive shapes is not necessary. The upper vertex $B \rightarrow D_{s}^{+} Y$ decays, which as well have an uncertainty of 12 to $15 \%$, are the other main contribution to the total uncertainty arising from background. All other background channels yield a negligible uncertainty.

\subsubsection{Particle Identification}

We use particle identification for the charged leptons, kaons, and protons. Each selector has a different efficiency to identify a true lepton/kaon/proton and a different fake rate to misidentify a particle's type. These efficiencies and fake rates are evaluated on control samples by the PID AWG for data and Monte Carlo separately, as discussed in section 6.3.3. Differences in data and MC are corrected on MC by the so-called tweaking method, which deletes candidates from or adds them to the set of identified candidates with the probability given by the efficiency ratio.

The main influence of particle identification in this analysis is on the background from fake muons (see figure 6.11 on page 60, yellow component). We estimate the uncertainty on this background by changing the tweaking probability for the muon selector within the statistical uncertainties of the identification efficiencies $\left(\Delta \varepsilon_{\mathrm{data} / \mathrm{MC}}\right)$ such that the difference of efficiencies between data and MC is maximized,

$$
\begin{aligned}
& \varepsilon_{\text {data }} \rightarrow \varepsilon_{\text {data }}-\Delta \varepsilon_{\text {data }} \text { and } \varepsilon_{\mathrm{MC}} \rightarrow \varepsilon_{\mathrm{MC}}+\Delta \varepsilon_{\mathrm{MC}}, \text { if } \varepsilon_{\text {data }}<\varepsilon_{\mathrm{MC}} \\
& \varepsilon_{\text {data }} \rightarrow \varepsilon_{\text {data }}+\Delta \varepsilon_{\text {data }} \text { and } \varepsilon_{\mathrm{MC}} \rightarrow \varepsilon_{\mathrm{MC}}-\Delta \varepsilon_{\mathrm{MC}}, \text { if } \varepsilon_{\text {data }}>\varepsilon_{\mathrm{MC}}
\end{aligned}
$$

As shown in figure 7.2, the size of the background component arising from fake muons changes up to $8 \%$. The shape is compatible with the one of the original component within uncertainties. We derive the systematic uncertainty due to particle identification by varying the fake muon background by $+8 \%$ and $-8 \%$ and extracting new sets of moments. The observed variation is taken as systematic uncertainty. 


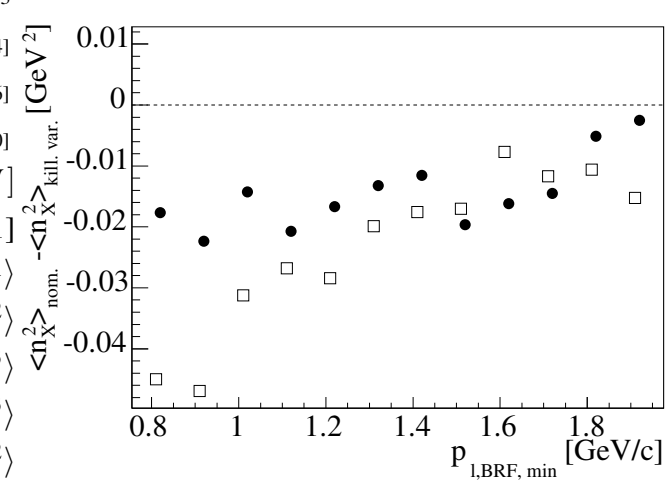

Figure 7.3: Variation of moments $\left\langle n_{X}^{2}\right\rangle$ with track $(\bullet)$ and neutral $(\square)$ killing on MC varied within their uncertainties; the difference between the nominal result and the one with varied killing probability is plotted. It depends on the minimal $p_{\ell, \mathrm{BRF}}$.

\subsubsection{Track Selection Efficiency}

The tracking algorithm used to find tracks from DCH and SVT hits has a different finding efficiency for simulated charged tracks than for data charged tracks in the detector. The ratio of efficiencies $R(\varepsilon)=\varepsilon_{\text {data }} / \varepsilon_{\mathrm{MC}}$ has been evaluated on a control sample of $e^{+} e^{-}$ $\rightarrow \tau^{+} \tau^{-}$decays by the BABAR tracking group. They give a difference in the track selection efficiency of about $0.5 \%$ with a systematic uncertainty of about $0.7 \%$. The efficiency differences in data and MC and their statistical uncertainties, arising from the limited control sample statistics, vary with azimuthal angle $\theta$, the polar angle $\phi$, the transverse momentum $p_{t}$, and the track multiplicity. They are tabulated and read out within the BABAR computing framework. We apply this nominal correction to the MC dataset by killing tracks randomly in the recoil of the $B_{\text {reco }}$ and the lepton with the given probability $p=1-R(\varepsilon)$. The systematic uncertainty arising from this difference is evaluated by changing the killing probability to $p^{\prime}=1-R^{\prime}(\varepsilon)$ with $R^{\prime}(\varepsilon)=R(\varepsilon)+\sqrt{\sigma_{\mathrm{R}, \text { stat. }}^{2}+(0.7 \%)^{2}}$. We assume that the error is symmetric, however, it is not possible to add tracks to the measured $X_{c}$ system. The systematic uncertainty is illustrated in figure 7.3 and listed in the summary tables 7.2 to 7.4 . It is the second largest systematic uncertainty for most cuts on the lepton momentum.

\subsubsection{Photon Selection Efficiency}

A similar situation as for the track selection efficiency is valid for the selection efficiency of photons. The difference in this efficiency between data and MC has been studied on various control samples, e.g. decays $D^{0} \rightarrow K \pi \pi^{0}$ [96] or $B$-meson decays $B^{0} \rightarrow D^{*-} \pi^{+}$ with the subsequent decay $D^{*-} \rightarrow D^{-} \pi^{0}$ [97], producing a sample of low energetic $\pi^{0}$ mesons. The study recommended by the BABAR working group for neutral particles uses a sample of $\tau$ decays, $\tau^{ \pm} \rightarrow \rho^{ \pm}\left(\rightarrow \pi^{ \pm} \pi^{0}\right) v_{\tau}$ and to $\tau^{ \pm} \rightarrow \pi^{ \pm} v_{\tau}$ [98]. All methods yield consistent results and are sensitive to the $\pi^{0}$ reconstruction from two photons and thus to the photon selection efficiency.

Flat Photon Efficiency Correction The BABAR neutral particles working group gives no nominal correction for the efficiency to reconstruct neutral particles. No energy dependence of the efficiency is observed. However, the analysis on the $\tau$ control sample has a systematic uncertainty of $1.8 \%$ originating from the analysis method. The systematic uncertainty on the moments measurement is evaluated by killing photons in the recoil of 
the $B_{\text {reco }}$ and the lepton with a probability of $1.8 \%$ independent of the photon energy and repeating the analysis. Figure 7.3 shows the variation of the extracted moments when turning this photon killing on. The variation is taken as systematic uncertainty. It is one of the dominant uncertainties, especially at low cuts on $p_{\ell, \mathrm{BRF}}$.

Energy Dependent Photon Killing Although the official recipe by the neutral AWG quotes no nominal correction, table 10 on page 34 in reference [98] shows an energy dependent difference in the selection efficiency $(0.1$ to $1.6 \%)$ for our photon energy range. Thus, we repeat our analysis with this energy dependent photon killing. The variation of the extracted moments is comparable to the one obtained from the flat killing of $1.8 \%$. Thus, no additional uncertainty is added.

\subsubsection{Imperfection of the Moment Extraction Method}

As pointed out in section 6.8.3, the calibration method used to extract the moments on data, suffers from small non-linearities of the calibration curves. Therefore, it leaves a bias after the calibration. We add half of the difference between the bias corrected moment on data and the moment without applying the final bias correction as a conservative systematic uncertainty on the extraction method.

\subsubsection{Simulation Model of Signal Decays}

The extraction of the moments on data relies on the signal model implemented in our MC simulations. The simulation enters into the building of the calibration curves (built on Cocktail MC) and into the extraction of the bias correction factors (extracted from generic $B \bar{B} \mathrm{MC})$. The signal branching fractions are the same for both datasets and are adjusted to the current measurements as pointed out in section 6.1. Thus, the dependence of the combined extraction method, that is the calibration and bias correction, on these branching fractions is evaluated. We rescale the eight exclusive $b \rightarrow c \ell v$ decay mode branching fractions in both MC datasets by six different numbers listed in table 7.1,

$$
\frac{\Gamma_{i}}{\Gamma_{\text {tot }, b \rightarrow c \ell v}}=s_{i, \text { nom }} \cdot s_{i, j} \cdot \frac{\Gamma_{i}}{\Gamma_{\text {tot }, b \rightarrow c \ell v}}, \quad \text { where } i \text { denotes the excl. channel and } j=1 \ldots 6 \text {. }
$$

The scaling factors $s_{i, j}$ have been randomly generated according to a Gaussian distribution with mean one and the widths given in the second column of the table. These widths are approximate numbers estimated from the uncertainties of the measured branching fractions as reported in table 6.1.

As the total semileptonic branching fraction is known with much better precision [42], two methods are used to keep it constant. Firstly, we rescale one single exclusive mode by one scale factor $s_{i, j}$, and rescale all other modes to keep the total rate constant. Secondly, we rescale all $B \rightarrow D^{* *} \ell v$ modes simultaneously with the same random scaling factor and compensate with the non-resonant components only and vice versa. The second method leaves the branching fractions of the decays $B \rightarrow D \ell v$ and $B \rightarrow D^{*} \ell v$ constant. 
Table 7.1: Variation factors of the relative branching fractions for exclusive decay modes. They are generated randomly according to a Gaussian distribution with mean one and $\sigma$ as given in the table. Negative scaling factors are set to zero.

\begin{tabular}{lllllllr}
\hline mode $i$ & $\begin{array}{l}\sigma \text { of } \\
\text { Gaussian }\end{array}$ & \multicolumn{6}{c}{$s_{i, j}$} \\
& 0.08 & 0.819 & 0.944 & 0.872 & 0.987 & 1.121 & 1.008 \\
$B \rightarrow D \ell v$ & 0.05 & 0.993 & 0.998 & 0.950 & 1.074 & 0.981 & 1.055 \\
$B \rightarrow D^{*} \ell v$ & 0.5 & 0.337 & 1.820 & 1.291 & 0.762 & 1.525 & 0.244 \\
\hline$B \rightarrow D_{1} \ell v$ & 0.5 & 0.280 & 0.511 & 0.385 & 0.608 & 0.861 & 1.521 \\
$B \rightarrow D_{2}^{*} \ell v$ & 0.5 & 1.337 & 1.418 & 0.330 & 1.074 & 0.867 & 1.659 \\
$B \rightarrow D_{0}^{*} \ell v$ & 0.5 & 0.754 & 1.812 & 1.236 & 0.560 & 1.795 & 0.429 \\
$B \rightarrow D_{1}^{\prime} \ell v$ & 0.5 & 2.272 & 0.221 & 0.752 & 0.508 & 1.123 & 0.975 \\
$B \rightarrow D \pi \ell v$ & 0.5 & 0.662 & 0.288 & 0.642 & 0.932 & 1.718 & 0.0 \\
$B \rightarrow D^{*} \pi \ell v$ & 0.5 & 1.281 & 0.705 & 1.212 & 0.017 & 0.016 & 0.257 \\
\hline all $D^{* *}$ modes & 0.5 & 0.761 & 0.966 & 1.431 & 1.504 & 0.794 & 0.568 \\
all non-res modes & 0.5 & & & & & &
\end{tabular}

With these modified signal compositions, 48 different in total, we extract new calibration curves, bias correction factors, and new sets of moments on data. The RMS of these distributions of the moments on data is taken as systematic uncertainty due to the modeling of signal decays. Figure 7.4 shows these distributions for different cuts on the lepton momentum. The derived uncertainty is small compared to the statistical uncertainties.

\subsubsection{Binning of Calibration Curves}

As discussed in section 6.9.1, we observe differences between the distributions of the charged and neutral multiplicity in data and MC simulations. The differences are more evident for the neutral multiplicity, however, they only appear for low photon energies below $100 \mathrm{MeV}$. In the charged multiplicity, we observe an overestimated number of events with $N_{\text {trk }, X}=2$ in data.

Differences in the $X_{c}$ multiplicity distributions and the $E_{\text {miss }}-c\left|\vec{p}_{\text {miss }}\right|$ distributions between data and MC may lead to the wrong choice of calibration curve, as these are binned in these two quantities. A possible impact of the mismodeling of the $E_{\text {miss }}-c\left|\vec{p}_{\text {miss }}\right|$ distribution is covered in the next subsection. Part of the differences visible in the multiplicity distributions may also arise from different selection efficiencies in data and MC, respectively, and are, therefore, already covered by the studies described in section 7.1 .5 and 7.1.6. This impact has been studied and a small difference between the multiplicity distributions remains even after applying the different killing probabilities on MC as described in these sections.

For the neutral multiplicity, this can be eliminated by changing the data multiplicity randomly in $7 \%$ of the events to $N_{\gamma, \text { mod }}=N_{\gamma \text {,meas. }}+1$. Using this smearing in the choice of the calibration curve leads to a new set of moments. A similar procedure is performed for the charged multiplicity by distributing $8 \%$ of the events with $N_{\text {trk,X }}=2$ to the neighbor bins ( $80 \%$ of the events to $N_{\text {trk }, X}+1,1 \%$ to $N_{\text {trk }, X}+2$, and $19 \%$ to $\left.N_{\text {trk }, X}+3\right)$. However, the effect on the moments on data is negligible compared to the smearing of the neutral 

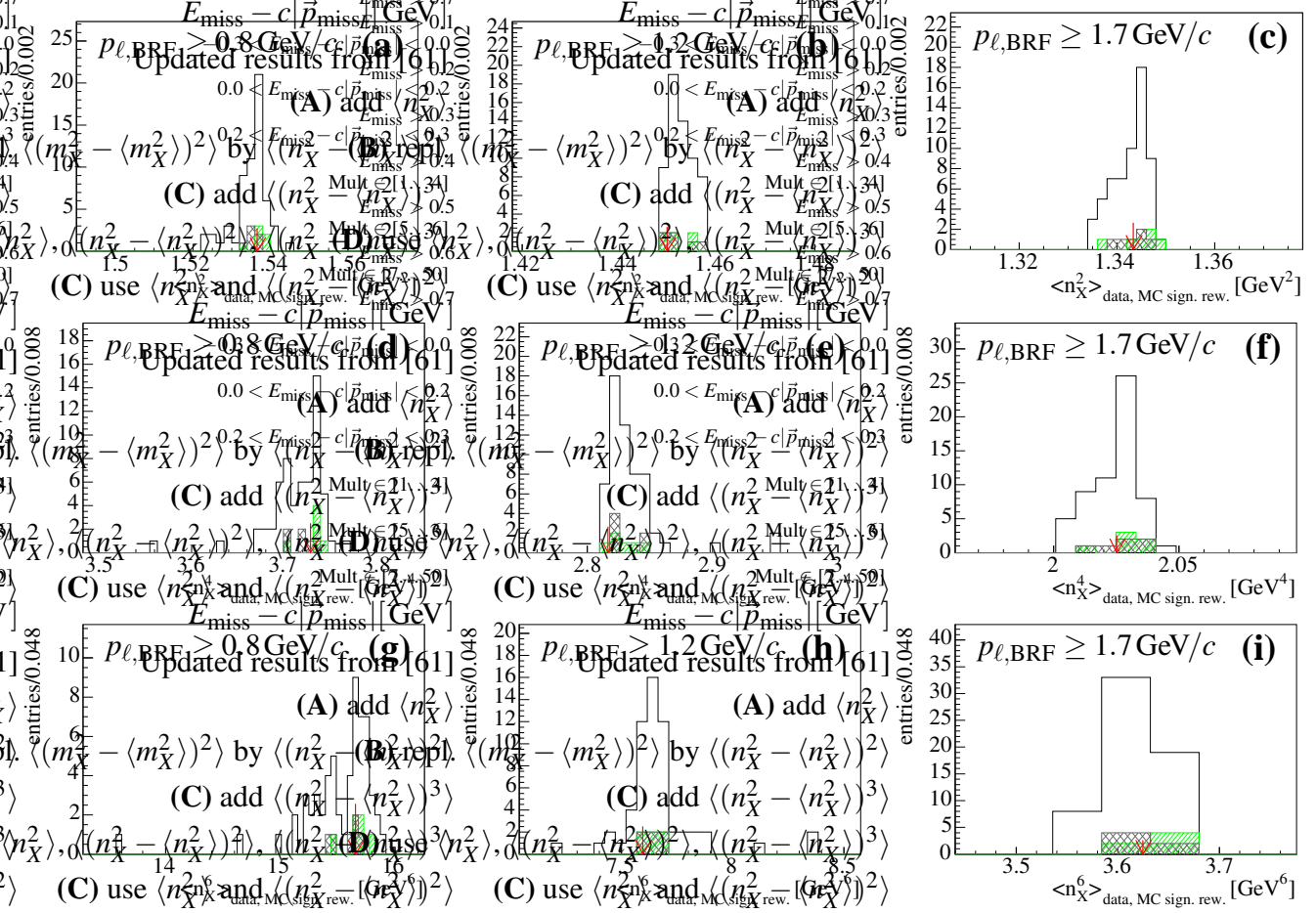

Figure 7.4: Systematic variation due to reweighting of signal branching fractions; (a-c) for moments $\left\langle n_{X}^{2}\right\rangle$, (d-f) for $\left\langle n_{X}^{4}\right\rangle$, and (g-i) for $\left\langle n_{X}^{6}\right\rangle$. The green, hatched component shows the effect from the variation of $\mathcal{B}(B \rightarrow D \ell v)$, the gray, crossed component shows the effect from the variation of the $B \rightarrow D^{*} \ell v$ component. The red arrow marks the nominal result. 

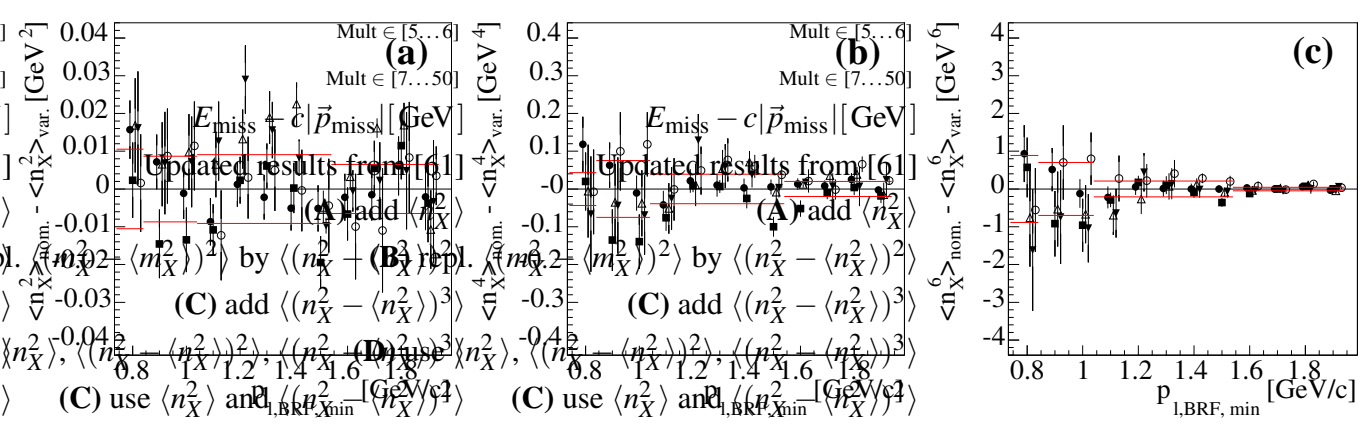

Figure 7.5: Impact of the $E_{\text {miss }}-c\left|\vec{p}_{\text {miss }}\right|$ cut variation. The difference to the nominal result is plotted, (a) for $\left\langle n_{X}^{2}\right\rangle$, (b) for $\left\langle n_{X}^{4}\right\rangle$, and (c) for $\left\langle n_{X}^{6}\right\rangle$. Five variation are performed: $-0.3<$ $E_{\text {miss }}-c\left|\vec{p}_{\text {miss }}\right|<0.3 \mathrm{GeV}(\bullet),-0.1<E_{\text {miss }}-c\left|\vec{p}_{\text {miss }}\right|<0.3 \mathrm{GeV}(\boldsymbol{\square}),-0.2<E_{\text {miss }}-c\left|\vec{p}_{\text {miss }}\right|$ $<0.5 \mathrm{GeV}(\triangle),-0.2<E_{\text {miss }}-c\left|\vec{p}_{\text {miss }}\right|<0.4 \mathrm{GeV}(\boldsymbol{\nabla}),-0.2<E_{\text {miss }}-c\left|\vec{p}_{\text {miss }}\right|<0.25 \mathrm{GeV}$ (०). The red lines indicate the assigned uncertainty.

multiplicity. We use the smearing in neutral and charged multiplicity simultaneously for the extraction of the systematic uncertainty, which is estimated by the shift of the measured moments. The estimated uncertainty is in the order of $0.007 \mathrm{GeV}^{2}$ for the moments $\left\langle n_{X}^{2}\right\rangle$ and, thus, smaller than other uncertainties, e.g. the one arising from the neutral selection efficiency.

\subsubsection{Mismodeling of $\mathbf{E}_{\text {miss }}-\mathbf{c} \overrightarrow{\mathbf{p}}_{\text {miss }}$}

The measurement of the mixed moments of the variable $n_{X}^{2}$ requires a narrow cut on $E_{\text {miss }}-$ $c\left|\vec{p}_{\text {miss }}\right|$ between -0.2 and $0.3 \mathrm{GeV}$. We, therefore, assign a systematic uncertainty due to possible mismodeling of the $E_{\text {miss }}-c\left|\vec{p}_{\text {miss }}\right|$ distribution in our simulation.

To get an estimate for this uncertainty, we vary the upper cut of $0.3 \mathrm{GeV}$ around the nominal value to $0.25,0.4$, and $0.5 \mathrm{GeV}$, leaving the lower cut at its nominal position. Then, we vary the lower cut at $-0.2 \mathrm{GeV}$ to -0.3 and $-0.1 \mathrm{GeV}$, leaving the upper one at $0.3 \mathrm{GeV}$. The binning of the calibration curves is adjusted accordingly. We find deviations in the measured moments on data and take the mean observed difference as systematic uncertainty. We smooth statistical fluctuations of the observed difference by taking the mean absolute difference in regions of $p_{\ell, \mathrm{BRF}}$, where the measured differences are of comparable size. They are indicated by the lines in figure 7.5 , where the observed differences are shown. This uncertainty is one of the larger ones, and for most cuts on $p_{\ell, \mathrm{BRF}}$ the third largest after the neutral and track selection efficiency .

\subsubsection{Impact of Final State Radiation}

To study the effect of final state radiation simulated by PHOTOS on the analysis, all events containing a photon generated by PHOTOS are excluded from the MC samples during the extraction of the calibration curves and bias correction factors. The moments on data are re-extracted using these modified calibration curves and bias correction factors. We find a significant deviation for the moments $\left\langle n_{X}^{2}\right\rangle$, while the deviation becomes negligible 

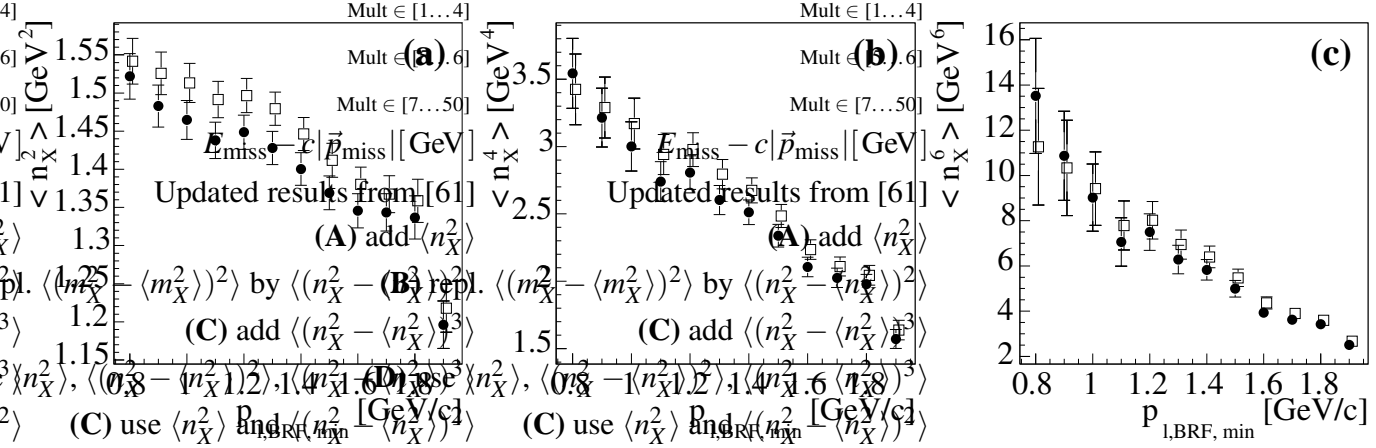

Figure 7.6: Comparison of nominal moments $(\bullet)$ with moments on data when excluding FSR events from the MC samples ( $\square$ ). Plot (a) shows the moments $\left\langle n_{X}^{2}\right\rangle$, (b) $\left\langle n_{X}^{4}\right\rangle$, and plot (c) $\left\langle n_{X}^{6}\right\rangle$.

compared to other uncertainties for the higher order moments above $p_{\ell, \mathrm{BRF}} \geq 0.9 \mathrm{GeV} / c$, see figure 7.6 for the comparison plots. As the total observed difference would overestimate the uncertainty, we take $20 \%$ of the observed difference as systematic uncertainty. This number follows estimates of uncertainties on the theoretical calculations that went into PHOTOS, and are recommended by the semileptonic AWG [99].

\subsection{Summary of Systematic Uncertainties}

Tables 7.2 to 7.4 summarize the central values, statistical and systematic uncertainties for the moments $\left\langle n_{X}^{2}\right\rangle,\left\langle n_{X}^{4}\right\rangle$, and $\left\langle n_{X}^{6}\right\rangle$. For the moments $\left\langle n_{X}^{2}\right\rangle$, the statistical uncertainty is in the order of $0.03 \mathrm{GeV}^{2}$ plus an additional contribution from the combinatorial background subtraction of the same order. The systematic uncertainty arising from the limited MC data sample is smaller by a factor of two. The combined systematic uncertainty is larger than the statistical error by roughly a factor of 2.5 . We identify the following dominant contributions to the systematic uncertainty:

- The neutral selection efficiency estimated by killing photons with a probability of $1.8 \%$ from the $X_{c}$ system is the dominant source of systematic uncertainties. It gets smaller for higher cuts on the lepton momentum. The track selection efficiency is of similar size in this region.

- The conservative estimate to add half of the applied bias correction as systematic uncertainty becomes the largest contribution to the uncertainty on $\left\langle n_{X}^{2}\right\rangle$ at high cuts on $p_{\ell, \mathrm{BRF}}$.

- The modeling of $E_{\text {miss }}-c\left|\vec{p}_{\text {miss }}\right|$ in the MC simulations derived from stability studies when changing the selected region in this variable contributes as the third largest error for most cuts on $p_{\ell, \mathrm{BRF}}$.

- The binning of the calibration curves in the multiplicity of the $X_{c}$ system, estimated by smearing the multiplicity within observed differences during the selection of the calibration curve is also sizable but not dominant an any cut on $p_{\ell, \mathrm{BRF}}$. 
Table 7.2: Central values and uncertainties for the $\left\langle n_{X}^{2}\right\rangle$ for all cuts on the lepton momentum. All uncertainties are given in $\mathrm{GeV}^{2}$.

\begin{tabular}{|c|c|c|c|c|c|c|c|c|c|c|c|c|}
\hline$p_{\ell, \mathrm{BRF}, \min }[\mathrm{GeV} / c]$ & 0.8 & 0.9 & 1.0 & 1.1 & 1.2 & 1.3 & 1.4 & 1.5 & 1.6 & 1.7 & 1.8 & 1.9 \\
\hline$\left\langle n_{X}^{2}\right\rangle\left[\mathrm{GeV}^{2}\right]$ & 1.522 & 1.483 & 1.465 & 1.438 & 1.449 & 1.428 & 1.400 & 1.369 & 1.346 & 1.343 & 1.337 & 1.196 \\
\hline Stat. error data & 0.030 & 0.028 & 0.025 & 0.024 & 0.022 & 0.022 & 0.021 & 0.022 & 0.022 & 0.024 & 0.028 & 0.031 \\
\hline Stat. error MC (calib.) & 0.008 & 0.007 & 0.007 & 0.007 & 0.007 & 0.008 & 0.008 & 0.008 & 0.008 & 0.010 & 0.012 & 0.012 \\
\hline Stat. error MC (residual bg. sub.) & 0.018 & 0.013 & 0.011 & 0.010 & 0.009 & 0.006 & 0.005 & 0.004 & 0.004 & 0.003 & 0.006 & 0.012 \\
\hline$B^{(+, 0)} \rightarrow \bar{D}^{0} \rightarrow e^{-}$ & 0.0011 & 0.0 & 0.0010 & 0.0 & 0.0011 & 0.0 & 0.0 & 0.0 & 0.0 & 0.0 & 0.0 & 0.0 \\
\hline$B^{(+, 0)} \rightarrow D^{+} \rightarrow e^{+}$ & 0.0010 & 0.0 & 0.0 & 0.0 & 0.0 & 0.0 & 0.0 & 0.0 & 0.0 & 0.0 & 0.0 & 0.0 \\
\hline$B^{(+, 0)} \rightarrow D_{s}^{+} \rightarrow e^{+}$ & 0.011 & 0.006 & 0.005 & 0.0016 & 0.0020 & 0.0011 & 0.0013 & 0.0 & 0.0 & 0.0 & 0.0 & 0.0 \\
\hline$B^{(+, 0)} \rightarrow \tau^{+} \rightarrow e^{+}$ & 0.0 & 0.0 & 0.0 & 0.0 & 0.0 & 0.0 & 0.0 & 0.0 & 0.0 & 0.0 & 0.0 & 0.0 \\
\hline$B^{(+, 0)} \rightarrow D_{s}^{+} \rightarrow \tau^{+} \rightarrow e^{+}$ & 0.0 & 0.0 & 0.0 & 0.0 & 0.0 & 0.0 & 0.0 & 0.0 & 0.0 & 0.0 & 0.0 & 0.0 \\
\hline$B(\rightarrow X) \rightarrow J / \Psi \rightarrow e^{+} e^{-}$, rightsign $e$ & 0.0 & 0.0 & 0.0 & 0.0 & 0.0 & 0.0 & 0.0 & 0.0 & 0.0 & 0.0 & 0.0 & 0.0 \\
\hline Bias corr. $1 / 2$ of corr. & 0.008 & 0.006 & 0.006 & 0.006 & 0.0015 & 0.0015 & 0.004 & 0.006 & 0.012 & 0.015 & 0.028 & 0.019 \\
\hline Sig. branching fractions & 0.004 & 0.004 & 0.003 & 0.0028 & 0.0025 & 0.004 & 0.004 & 0.005 & 0.003 & 0.004 & 0.004 & 0.003 \\
\hline Calib. curve selection (mult.) & 0.007 & 0.007 & 0.007 & 0.007 & 0.006 & 0.006 & 0.005 & 0.005 & 0.006 & 0.005 & 0.006 & 0.005 \\
\hline$\left|E_{\text {miss }}-c\right| \vec{p}_{\text {miss }}||$ mismodelling & 0.011 & 0.009 & 0.009 & 0.009 & 0.009 & 0.009 & 0.009 & 0.009 & 0.006 & 0.006 & 0.006 & 0.006 \\
\hline FSR by PHOTOS & 0.004 & 0.009 & 0.010 & 0.011 & 0.010 & 0.010 & 0.009 & 0.009 & 0.007 & 0.005 & 0.004 & 0.004 \\
\hline Sys. error total & 0.055 & 0.057 & 0.041 & 0.040 & 0.038 & 0.030 & 0.027 & 0.031 & 0.026 & 0.027 & 0.033 & 0.030 \\
\hline Error total & 0.074 & 0.074 & 0.060 & 0.054 & 0.051 & 0.044 & 0.041 & 0.047 & 0.043 & 0.047 & 0.052 & 0.046 \\
\hline
\end{tabular}


Table 7.3: Central values and uncertainties for the moments $\left\langle n_{X}^{4}\right\rangle$ for all cuts on the lepton momentum. All uncertainties are given in $\mathrm{GeV}^{4}$.

\begin{tabular}{|c|c|c|c|c|c|c|c|c|c|c|c|c|}
\hline$p_{\ell, \mathrm{BRF}, \min }[\mathrm{GeV} / c]$ & 0.8 & 0.9 & 1.0 & 1.1 & 1.2 & 1.3 & 1.4 & 1.5 & 1.6 & 1.7 & 1.8 & 1.9 \\
\hline$\left\langle n_{X}^{4}\right\rangle\left[\mathrm{GeV}^{4}\right]$ & 3.54 & 3.21 & 3.00 & 2.74 & 2.81 & 2.60 & 2.51 & 2.34 & 2.11 & 2.03 & 1.98 & 1.57 \\
\hline Stat. error data & 0.26 & 0.22 & 0.18 & 0.15 & 0.12 & 0.11 & 0.09 & 0.08 & 0.07 & 0.07 & 0.07 & 0.07 \\
\hline Stat. error data (comb. bg. sub.) & 0.32 & 0.29 & 0.22 & 0.16 & 0.14 & 0.11 & 0.09 & 0.10 & 0.07 & 0.10 & 0.07 & 0.011 \\
\hline Stat. error MC (calib.) & 0.06 & 0.06 & 0.05 & 0.03 & 0.04 & 0.03 & 0.028 & 0.026 & 0.026 & 0.027 & 0.04 & 0.03 \\
\hline Stat. error MC (residual bg. sub.) & 0.13 & 0.10 & 0.08 & 0.05 & 0.05 & 0.03 & 0.027 & 0.022 & 0.019 & 0.0 & 0.0 & 0.0 \\
\hline$B \rightarrow X_{u} \ell v(\mathrm{Res})$ & 0.0 & 0.012 & 0.0 & 0.0 & 0.0 & 0.0 & 0.0 & 0.0 & 0.0 & 0.0 & 0.0 & 0.0 \\
\hline$B^{(+, 0)} \rightarrow D^{0} \rightarrow e^{+}$ & 0.013 & 0.0 & 0.0 & 0.0 & 0.0 & 0.0 & 0.0 & 0.0 & 0.0 & 0.0 & 0.0 & 0.0 \\
\hline$B^{(+, 0)} \rightarrow \bar{D}^{0} \rightarrow e^{-}$ & 0.011 & 0.0 & 0.0 & 0.0 & 0.0 & 0.0 & 0.0 & 0.0 & 0.0 & 0.0 & 0.0 & 0.0 \\
\hline$B^{(+, 0)} \rightarrow D^{+} \rightarrow e^{+}$ & 0.011 & 0.0 & 0.0 & 0.0 & 0.0 & 0.0 & 0.0 & 0.0 & 0.0 & 0.0 & 0.0 & 0.0 \\
\hline$B^{(+, 0)} \rightarrow D_{s}^{+} \rightarrow e^{+}$ & 0.09 & 0.05 & 0.04 & 0.010 & 0.013 & 0.0 & 0.0 & 0.0 & 0.0 & 0.0 & 0.0 & 0.0 \\
\hline$B^{(+, 0)} \rightarrow \tau^{+} \rightarrow e^{+}$ & 0.0 & 0.0 & 0.0 & 0.0 & 0.0 & 0.0 & 0.0 & 0.0 & 0.0 & 0.0 & 0.0 & 0.0 \\
\hline$B^{(+, 0)} \rightarrow D_{s}^{+} \rightarrow \tau^{+} \rightarrow e^{+}$ & 0.0 & 0.0 & 0.0 & 0.0 & 0.0 & 0.0 & 0.0 & 0.0 & 0.0 & 0.0 & 0.0 & 0.0 \\
\hline$B(\rightarrow X) \rightarrow J / \Psi \rightarrow e^{+} e^{-}$, rightsign $e$ & 0.0 & 0.0 & 0.0 & 0.0 & 0.0 & 0.0 & 0.0 & 0.0 & 0.0 & 0.0 & 0.0 & 0.0 \\
\hline$B(\rightarrow X) \rightarrow \Psi(2 S) \rightarrow e^{+} e^{-}$, rightsign $e$ & 0.0 & 0.0 & 0.0 & 0.0 & 0.0 & 0.0 & 0.0 & 0.0 & 0.0 & 0.0 & 0.0 & 0.0 \\
\hline PID fake lepton bg. & 0.026 & 0.0 & 0.0 & 0.0 & 0.0 & 0.0 & 0.0 & 0.0 & 0.0 & 0.0 & 0.0 & 0.0 \\
\hline Trk. sel. eff. (killing) & 0.08 & 0.10 & 0.05 & 0.06 & 0.04 & 0.021 & 0.013 & 0.04 & 0.027 & 0.03 & 0.0 & 0.0 \\
\hline Neutral sel. eff. (killing) & 0.32 & 0.30 & 0.19 & 0.11 & 0.13 & 0.08 & 0.06 & 0.06 & 0.04 & 0.04 & 0.04 & 0.04 \\
\hline Bias corr. $1 / 2$ of corr. & 0.07 & 0.09 & 0.09 & 0.08 & 0.12 & 0.10 & 0.08 & 0.09 & 0.06 & 0.04 & 0.019 & 0.019 \\
\hline Sig. branching fractions & 0.03 & 0.023 & 0.021 & 0.021 & 0.026 & 0.04 & 0.026 & 0.018 & 0.0 & 0.0 & 0.013 & 0.0 \\
\hline Calib. curve selection (mult.) & 0.021 & 0.020 & 0.018 & 0.019 & 0.017 & 0.016 & 0.012 & 0.010 & 0.010 & 0.0 & 0.0 & 0.0 \\
\hline$\left|E_{\text {miss }}-c\right| \vec{p}_{\text {miss }}||$ mismodelling & 0.04 & 0.07 & 0.07 & 0.04 & 0.04 & 0.04 & 0.04 & 0.04 & 0.020 & 0.020 & 0.020 & 0.020 \\
\hline FSR by PHOTOS & 0.024 & 0.015 & 0.03 & 0.04 & 0.03 & 0.04 & 0.03 & 0.029 & 0.026 & 0.017 & 0.013 & 0.013 \\
\hline Sys. error total & 0.38 & 0.35 & 0.25 & 0.17 & 0.20 & 0.15 & 0.12 & 0.13 & 0.09 & 0.07 & 0.05 & 0.05 \\
\hline Error total & 0.57 & 0.51 & 0.38 & 0.28 & 0.28 & 0.22 & 0.18 & 0.18 & 0.13 & 0.14 & 0.12 & 0.10 \\
\hline
\end{tabular}


Table 7.4: Central values and uncertainties for the moments $\left\langle n_{X}^{6}\right\rangle$ for all cuts on the lepton momentum. All uncertainties are given in $\mathrm{GeV}^{6}$.

\begin{tabular}{|c|c|c|c|c|c|c|c|c|c|c|c|c|}
\hline$p_{\ell, \mathrm{BRF}, \min }[\mathrm{GeV} / c]$ & 0.8 & 0.9 & 1.0 & 1.1 & 1.2 & 1.3 & 1.4 & 1.5 & 1.6 & 1.7 & 1.8 & 1.9 \\
\hline$\left\langle n_{X}^{6}\right\rangle\left[\mathrm{GeV}^{6}\right]$ & 13.52 & 10.87 & 9.02 & 7.06 & 7.50 & 6.28 & 5.83 & 4.99 & 3.93 & 3.63 & 3.42 & 2.51 \\
\hline Stat. error data & 2.54 & 1.97 & 1.49 & 1.07 & 0.81 & 0.63 & 0.45 & 0.37 & 0.26 & 0.22 & 0.19 & 0.16 \\
\hline Stat. error data (comb. bg. sub.) & 2.99 & 1.96 & 1.65 & 0.82 & 0.83 & 0.56 & 0.42 & 0.32 & 0.20 & 0.26 & 0.13 & 0.022 \\
\hline Stat. error MC (calib.) & 0.70 & 0.57 & 0.48 & 0.18 & 0.19 & 0.14 & 0.12 & 0.10 & 0.09 & 0.09 & 0.09 & 0.06 \\
\hline Stat. error MC (residual bg. sub.) & 1.18 & 0.73 & 0.69 & 0.31 & 0.26 & 0.17 & 0.11 & 0.08 & 0.07 & 0.019 & 0.013 & 0.019 \\
\hline$B \rightarrow X_{u} \ell v(\operatorname{Res})$ & 0.08 & 0.07 & 0.06 & 0.021 & 0.0 & 0.05 & 0.05 & 0.03 & 0.026 & 0.025 & 0.019 & 0.021 \\
\hline$B^{(+, 0)} \rightarrow D^{0} \rightarrow e^{+}$ & 0.11 & 0.06 & 0.015 & 0.0 & 0.0 & 0.0 & 0.0 & 0.0 & 0.0 & 0.0 & 0.0 & 0.0 \\
\hline$B^{(+, 0)} \rightarrow \bar{D}^{0} \rightarrow e^{-}$ & 0.10 & 0.0 & 0.03 & 0.03 & 0.05 & 0.0 & 0.015 & 0.0 & 0.0 & 0.0 & 0.0 & 0.0 \\
\hline$B^{(+, 0)} \rightarrow D^{+} \rightarrow e^{+}$ & 0.11 & 0.0 & 0.04 & 0.0 & 0.012 & 0.0 & 0.0 & 0.0 & 0.0 & 0.0 & 0.0 & 0.0 \\
\hline$B^{(+, 0)} \rightarrow D_{s}^{+} \rightarrow e^{+}$ & 0.78 & 0.35 & 0.27 & 0.06 & 0.08 & 0.04 & 0.03 & 0.0 & 0.0 & 0.0 & 0.0 & 0.0 \\
\hline$B^{(+, 0)} \rightarrow \tau^{+} \rightarrow e^{+}$ & 0.022 & 0.0 & 0.0 & 0.0 & 0.029 & 0.0 & 0.0 & 0.0 & 0.0 & 0.0 & 0.0 & 0.0 \\
\hline$B^{(+, 0)} \rightarrow D_{s}^{+} \rightarrow \tau^{+} \rightarrow e^{+}$ & 0.0 & 0.04 & 0.0 & 0.0 & 0.04 & 0.0 & 0.016 & 0.0 & 0.0 & 0.0 & 0.0 & 0.0 \\
\hline$B(\rightarrow X) \rightarrow J / \Psi \rightarrow e^{+} e^{-}$, rightsign $e$ & 0.0 & 0.030 & 0.011 & 0.0 & 0.023 & 0.0 & 0.0 & 0.0 & 0.0 & 0.0 & 0.0 & 0.0 \\
\hline$B(\rightarrow X) \rightarrow \Psi(2 S) \rightarrow e^{+} e^{-}$, rightsign $e$ & 0.0 & 0.0 & 0.0 & 0.0 & 0.0 & 0.0 & 0.0 & 0.0 & 0.0 & 0.0 & 0.0 & 0.0 \\
\hline PID fake lepton bg. & 0.24 & 0.019 & 0.0 & 0.021 & 0.015 & 0.018 & 0.020 & 0.0 & 0.0 & 0.0 & 0.0 & 0.0 \\
\hline Trk. sel. eff. (killing) & 0.69 & 0.73 & 0.35 & 0.23 & 0.15 & 0.05 & 0.015 & 0.10 & 0.06 & 0.09 & 0.029 & 0.0 \\
\hline Neutral sel. eff. (killing) & 2.70 & 2.16 & 1.34 & 0.47 & 0.61 & 0.28 & 0.17 & 0.17 & 0.10 & 0.04 & 0.09 & 0.09 \\
\hline Bias corr. $1 / 2$ of corr. & 0.48 & 0.52 & 0.54 & 0.34 & 0.48 & 0.41 & 0.32 & 0.40 & 0.24 & 0.15 & 0.12 & 0.024 \\
\hline Sig. branching fractions & 0.34 & 0.24 & 0.20 & 0.14 & 0.18 & 0.20 & 0.12 & 0.05 & 0.03 & 0.03 & 0.03 & 0.024 \\
\hline Calib. curve selection (mult.) & 0.07 & 0.07 & 0.06 & 0.07 & 0.06 & 0.05 & 0.03 & 0.025 & 0.025 & 0.018 & 0.020 & 0.013 \\
\hline$\left|E_{\text {miss }}-c\right| \vec{p}_{\text {miss }}||$ mismodelling & 0.89 & 0.70 & 0.70 & 0.21 & 0.21 & 0.21 & 0.21 & 0.21 & 0.05 & 0.05 & 0.05 & 0.05 \\
\hline FSR by PHOTOS & 0.45 & 0.11 & 0.08 & 0.15 & 0.10 & 0.13 & 0.11 & 0.10 & 0.09 & 0.05 & 0.04 & 0.03 \\
\hline Sys. error total & 3.35 & 2.59 & 1.82 & 0.76 & 0.90 & 0.62 & 0.47 & 0.51 & 0.30 & 0.20 & 0.17 & 0.12 \\
\hline Error total & 5.21 & 3.84 & 2.91 & 1.56 & 1.48 & 1.06 & 0.78 & 0.72 & 0.45 & 0.41 & 0.30 & 0.21 \\
\hline
\end{tabular}




\subsection{Stability Studies}

To test the stability of the extracted results, the data sample is divided into independent parts and the moments are extracted in each of these parts. We consider the following divisions:

- $B_{\text {reco }}$ candidates reconstructed as neutral and charged $B$ mesons, respectively. As background contributions, e.g. arising from $B^{0} \bar{B}^{0}$ oscillations, are different for these subsamples, this test is sensitive to their description.

- Semileptonic decays reconstructed using an identified muon or electron, respectively. Again, background contributions are significantly different for these disjoined data samples, as discussed for the fake muon background.

- Semileptonic decays reconstructed using a positively or negatively charged lepton. We do not expect any difference here.

- Data taken during Run 1-3 and during Run 4, respectively. As the luminosity and thus background conditions have been different for these runperiods, this study may reveal problems arising from these differences, which have shown up in the neutral multiplicity for low energetic photons.

As differences in the calibration curves for events with eletrons or muons became obvious, it is checked whether a similar situation is valid for the other considered subsets. As no significant difference is found, the usual method to use the two different types of calibration curves (for events with electrons and muons) is applied to all subsets.

The extracted moments for the subsets are in agreement within statistical uncertainties, as shown in figure 7.7 and 7.8. Whenever differences larger than one standard deviation are found, they are caused by statistical fluctuations in one bin of $p_{\ell, \mathrm{BRF}}$. We do not assign any further uncertainty to the measured moments. 

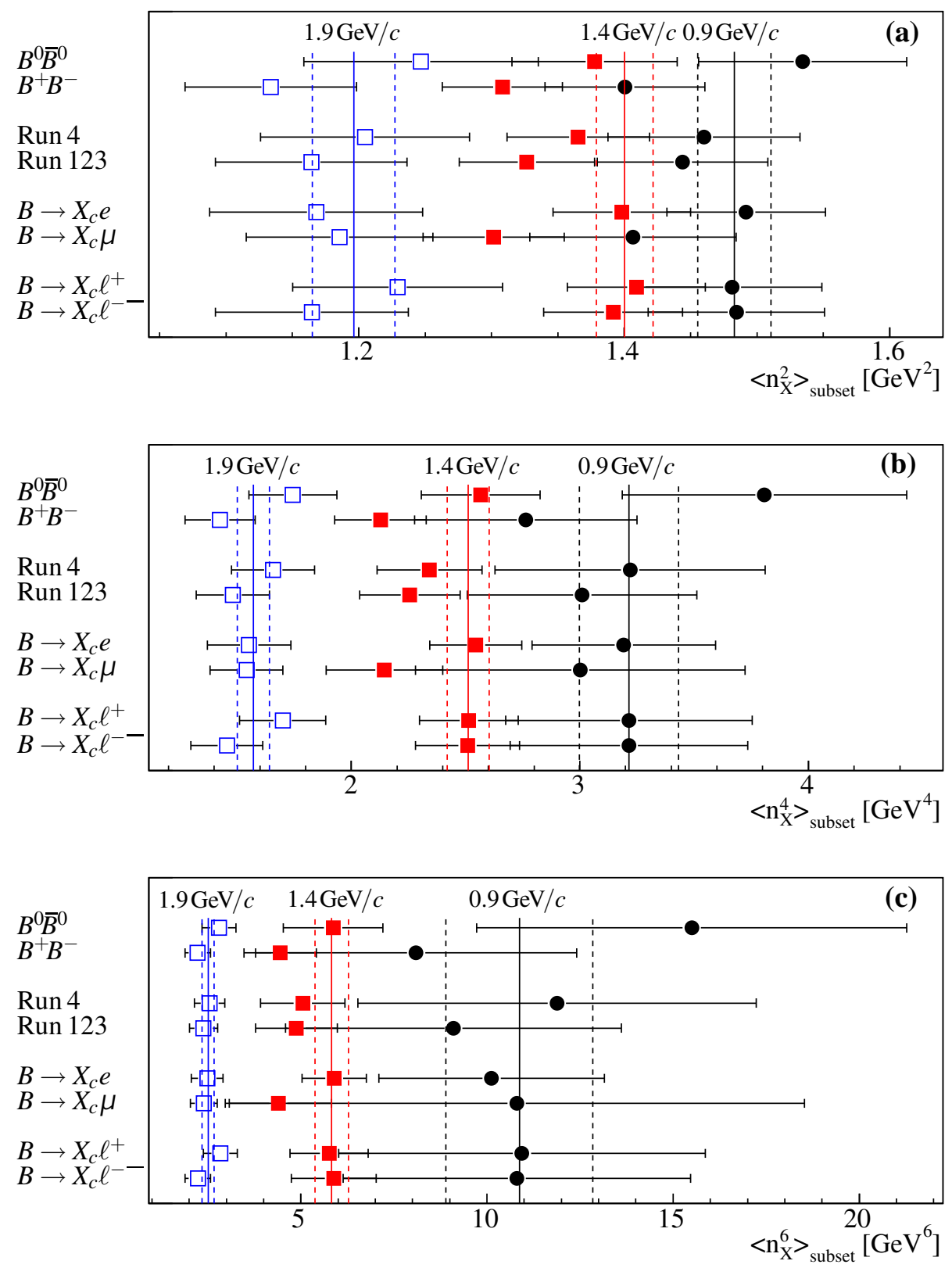

Figure 7.7: Moments of independent subsets on data. Plot (a) shows the moments $\left\langle n_{X}^{2}\right\rangle$, (b) $\left\langle n_{X}^{4}\right\rangle$, and (c) $\left\langle n_{X}^{6}\right\rangle$, all for three different cuts on $p_{\ell, \mathrm{BRF}}$, as indicated in the plots. The solid line marks the nominal moment with its statistical uncertainty (dashed lines). The error bars mark the statistical plus the systematic uncertainty arising from the background subtraction (combinatorial and residual). 

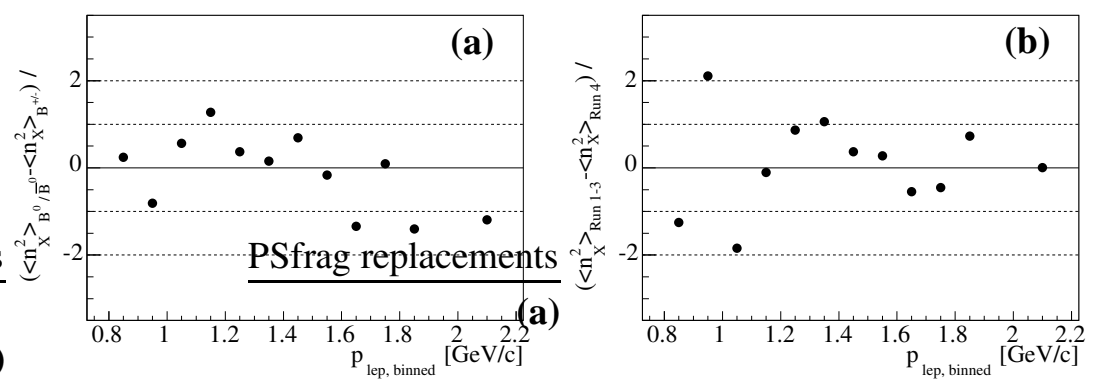

Figure 7.8: Normalized comparison of binned moments of independent subsets on data. (a) shows the comparison for charged and neutral $B$ mesons and (b) for the data recorded during Run 123 and Run 4, respectively. 


\section{Interpretation of the Measurement}

In this chapter the measured moments will be interpreted in the context of a Heavy Quark Expansion in the kinetic scheme. We extract results for the CKM-Matrix element $\left|V_{c b}\right|$, the quark masses $m_{b}$ and $m_{c}$, the semileptonic branching fraction $\mathcal{B}\left(B \rightarrow X_{c} \ell v\right)$, and the four non-perturbative HQE parameters $\mu_{\pi}^{2}, \mu_{G}^{2}, \rho_{\mathrm{LS}}^{3}$, and $\rho_{D}^{3}$ in a global fit combining the presented results with additional measurements of moments of the lepton-energy spectrum in decays $B \rightarrow X_{c} \ell v,\left\langle E_{\ell}^{k}\right\rangle$, and moments of the photon-energy spectrum in decays $B \rightarrow X_{s} \gamma$, $\left\langle E_{\gamma}^{k}\right\rangle$. Previous HQE fits used hadronic-mass moments instead of the moments of the $n_{X}^{2}$ distribution. Therefore, the effect of adding $\left\langle n_{X}^{k}\right\rangle$ moments and replacing hadronic-mass moments by the $\left\langle n_{X}^{k}\right\rangle$ moments is studied. For the final fit procedure we show verification studies and discuss the results.

\subsection{The Fit Idea}

At the parton level, the weak decay rate for $b \rightarrow c \ell v$ can be calculated accurately; it is proportional to $\left|V_{c b}\right|^{2}$ and depends on the quark masses $m_{b}$ and $m_{c}$. To relate measurements of the semileptonic $B$-meson decay rate to $\left|V_{c b}\right|$, the parton-level calculations have to be corrected for effects of strong interactions. This concept has been discussed in section 3.2.3.

In the kinetic-mass scheme $[6,32,100-103]$, these expansions in $1 / m_{b}$ and in the strong coupling constant $\alpha_{s}\left(m_{b}\right)$ to order $\mathcal{O}\left(1 / m_{b}^{3}\right)$ and $\mathcal{O}\left(\alpha_{s}^{2}\right)$ contain six parameters: the running kinetic masses of the $b$ - and $c$-quarks, $m_{b}(\mu)$ and $m_{c}(\mu)$, and four non-perturbative parameters, that are $\mu_{\pi}^{2}(\mu)$ and $\mu_{G}^{2}(\mu)$ appearing in the expansion up to order $1 / m_{b}^{2}$, and $\rho_{D}^{3}(\mu)$ and $\rho_{\mathrm{LS}}^{3}(\mu)$ appearing in the expansion up to order $1 / m_{b}^{3}$. The parameter $\mu$ denotes the renormalization scale that separates effects from long- and short-distance dynamics. The calculations are performed for $\mu=1 \mathrm{GeV}$ [104]. The expansion to $\mathcal{O}\left(1 / m_{b}^{3}\right)$ for the rate $\Gamma_{\mathrm{SL}}$ of decays $B \rightarrow X_{c} \ell v$ is given in eq. (3.36).

We determine these six parameters from a fit to moments of hadronic observables $\left(n_{X}^{2}\right.$ and $m_{X}$ ) and moments of the electron-energy spectrum in decays $B \rightarrow X_{c} \ell v$ [33]. Furthermore, we use moments of the photon-energy spectrum in decays $B \rightarrow X_{s} \gamma[105,106]$. The performed fit uses a linearized expression for the dependence of $\left|V_{c b}\right|$ on the values of heavy-quark parameters, expanded around a priori estimates of these parameters [32],

$$
\begin{aligned}
\frac{\left|V_{c b}\right|}{0.0417}= & \sqrt{\frac{\mathcal{B}_{c \ell v}}{0.1032} \frac{1.55}{\tau_{B}}} \\
& \times\left[1+0.30\left(\alpha_{s}\left(m_{b}\right)-0.22\right)\right] \\
& \times\left[1-0.66\left(m_{b}-4.60\right)+0.39\left(m_{c}-1.15\right)\right. \\
& +0.013\left(\mu_{\pi}^{2}-0.40\right)+0.09\left(\rho_{D}^{3}-0.20\right) \\
& \left.+0.05\left(\mu_{G}^{2}-0.35\right)-0.01\left(\rho_{\mathrm{LS}}^{3}+0.15\right)\right] .
\end{aligned}
$$


Here $m_{b}$ and $m_{c}$ are in units $\mathrm{GeV} / c^{2}$ and all other parameters of the expansion are in $\mathrm{GeV}^{k}$; $\tau_{B}$ refers to the average lifetime of $B$ mesons and is given in ps.

Expansions in terms of the same heavy-quark parameters are available for hadronicmass, hadronic- $n_{X}^{2}$, electron-energy, and photon-energy moments. Predictions for those moments are obtained from analytical calculations to compare them to the measurements, and thereby extract the best estimates for the parameters.

\subsection{Extraction Formalism}

The fit method designed to extract the HQE parameters from the moments measurements has been reported previously $[35,36]$. It is based on a $\chi^{2}$ minimization,

$$
\chi^{2}=\left(\vec{M}_{\mathrm{exp}}-\vec{M}_{\mathrm{HQE}}\right)^{T} \mathcal{C}_{\mathrm{tot}}^{-1}\left(\vec{M}_{\mathrm{exp}}-\vec{M}_{\mathrm{HQE}}\right) .
$$

The vectors $\vec{M}_{\text {exp }}$ and $\vec{M}_{\mathrm{HQE}}$ contain the measured moments included in the fit and the corresponding moments calculated by theory, respectively. Furthermore, the expression in eq. (8.2) contains the total covariance matrix $\mathcal{C}_{\text {tot }}$ defined as the sum of the experimental, $\mathcal{C}_{\text {exp }}$, and theoretical, $\mathcal{C}_{\mathrm{HQE}}$, covariance matrices (see section 8.3).

The semileptonic branching fraction, $\mathcal{B}\left(B \rightarrow X_{c} \ell v\right)$, is extracted in the fit by extrapolating measured partial branching fractions, $\mathcal{B}_{p_{\ell, \mathrm{BRF}, \min }}\left(B \rightarrow X_{c} \ell v\right)$, measured with a cut $p_{\ell, \mathrm{BRF}} \geq p_{\ell, \mathrm{BRF}, \mathrm{min}}$, to the full lepton energy spectrum. Using HQE predictions of the relative decay fraction

$$
R_{p_{\ell, \mathrm{BRF}, \min }}=\frac{\int_{p_{\ell, \mathrm{BRF}, \mathrm{min}}} \frac{\mathrm{d} \Gamma_{\mathrm{SL}}}{\mathrm{d} E_{\ell, \mathrm{BRF}}} \mathrm{d} E_{\ell, \mathrm{BRF}}}{\int_{0} \frac{\mathrm{d} \Gamma_{\mathrm{SL}}}{\mathrm{d} E_{\ell, \mathrm{BRF}}} \mathrm{d} E_{\ell, \mathrm{BRF}}},
$$

the total branching fraction can be introduced as a free parameter in the fit. It is given by

$$
\mathcal{B}\left(B \rightarrow X_{c} \ell v\right)=\frac{\mathcal{B}_{p_{\ell, \mathrm{BRF}, \min }}\left(B \rightarrow X_{c} \ell v\right)}{R_{p_{\ell, \mathrm{BRF}, \min }}} .
$$

The total branching fraction can be used together with the average $B$-meson lifetime $\tau_{B}$ to calculate the total semileptonic rate which is proportional to $\left|V_{c b}\right|^{2}$,

$$
\Gamma_{\mathrm{SL}}=\frac{\mathcal{B}\left(B \rightarrow X_{c} \ell v\right)}{\tau_{B}} \propto\left|V_{c b}\right|^{2}
$$

By adding $\tau_{B}$ to the vectors of measured and predicted quantities, $\vec{M}_{\text {exp }}$ and $\vec{M}_{\mathrm{HQE}},\left|V_{c b}\right|$ can be extracted from the fit as an additional free parameter using eq. (8.1).

While the non-perturbative parameters $\mu_{\pi}^{2}$ and $\rho_{D}^{3}$ are completely free in the fit, the parameters $\mu_{G}^{2}$ and $\rho_{\mathrm{LS}}^{3}$ have been estimated from $B-B^{*}$ mass splitting and heavy-quark sum rules to be $\mu_{G}^{2}=(0.35 \pm 0.07) \mathrm{GeV}^{2}$ and $\rho_{\mathrm{LS}}^{3}=(-0.15 \pm 0.10) \mathrm{GeV}^{3}$ [36], respectively. Both parameters are restricted in the fit by imposing Gaussian error constraints. 


\subsection{Theoretical Uncertainties}

As discussed in [36] and specified in [6] the following theoretical uncertainties are taken into account:

The uncertainty related to the uncalculated perturbative corrections to the Wilson coefficients of non-perturbative operators are estimated by varying the corresponding parameters $\mu_{\pi}^{2}$ and $\mu_{G}^{2}$ by $20 \%$ and $\rho_{D}^{3}$ and $\rho_{\mathrm{LS}}^{3}$ by $30 \%$ around their expected values.

Uncertainties for the perturbative corrections are estimated by varying $\alpha_{s}=0.22$ up and down by 0.1 for the hadronic mass moments and by 0.04 for the lepton energy moments around its nominal value.

Uncertainties in the perturbative corrections to the quark masses $m_{b}$ and $m_{c}$ are addressed by varying both by $20 \mathrm{MeV} / c^{2}$ up and down around their expected values.

All these uncertainties are evaluated dependent on the cut on the lepton momentum, resulting in theoretical uncertainties slightly increasing with this cut.

For the extracted value of $\left|V_{c b}\right|$ an additional error of $1.4 \%$ is added for the uncertainty in the expansion of the semileptonic rate $\Gamma_{\mathrm{SL}}[32,103]$. It accounts for remaining uncertainties in the perturbative corrections to the leading operator, uncalculated perturbative corrections to the chromomagnetic and Darwin operator, higher order power corrections, and possible non-perturbative effects in the operators with charm fields. This uncertainty is not included in the theoretical covariance matrix $\mathcal{C}_{\mathrm{HQE}}$ but is listed separately as a theoretical uncertainty on $\left|V_{c b}\right|$. Thereby, this uncertainty does not affect the fit result.

For the predicted photon energy moments $\left\langle E_{\gamma}^{k}\right\rangle$, additional uncertainties are taken into account. As outlined in [100], additional uncertainties of $30 \%$ of the applied bias correction to the photon-energy moments and half the difference in the moments derived from two different distribution-function ansätze have to be considered. Both contributions are added linearly.

The theoretical covariance matrix $\mathcal{C}_{\mathrm{HQE}}$ is constructed by assuming fully correlated theoretical uncertainties for a given moment with different lepton momentum or photon energy cutoff and assuming uncorrelated theoretical uncertainties for moments of different orders and types. The theoretical uncertainties of moments of the hadronic mass and of the $n_{X}^{2}$ spectrum of the same order, e. g. $\left\langle m_{X}^{2}\right\rangle$ and $\left\langle n_{X}^{2}\right\rangle$, are assumed to be $100 \%$ correlated. The additional uncertainties considered for the photon energy moments are assumed to be uncorrelated for different moments and photon energy cutoffs.

\subsection{Experimental Correlations between Mass and Mass-Energy Moments}

We investigate and discuss the impact of adding the moments of the $n_{X}^{2}$ spectrum to the fit or of replacing hadronic-mass moments with moments $\left\langle n_{X}^{k}\right\rangle$ as proposed by theorists [7]. For this inclusion of the measured moments $\left\langle n_{X}^{k}\right\rangle$ in the global HQE fit, correlations between the mass moments measured in [61] and the presented moments of the $n_{X}^{2}$ distribution are needed. As the mass enters directly into the calculation of $n_{X}^{2}$ according to eq. (1.1), the correlation is not negligible. Furthermore, the two analyses are performed on nearly the same dataset, where differences only arise due to a tighter requirement on $E_{\text {miss }}-c\left|\vec{p}_{\text {miss }}\right|$ in this analysis. We derive the correlations between two moments $\left.\left\langle n_{X}^{k}\right\rangle\right|_{p_{\ell, \mathrm{BRF}} \geq a \mathrm{GeV} / c}$ and $\left.\left\langle m_{X}^{l}\right\rangle\right|_{p_{\ell, \mathrm{BRF}} \geq b \mathrm{GeV} / c}$ from the correlation of the two-dimensional $n_{X}^{k}-m_{X}^{l}$ distribution mea- 
sured for the same cut on the lepton momentum and from the correlations derived for the $n_{X}^{k}$ and $m_{X}^{k}$ moments alone, which account for the overlapping data samples at different cuts on $p_{\ell, \mathrm{BRF}}$,

$$
\rho\left(\left.\left\langle n_{X}^{k}\right\rangle\right|_{a},\left.\left\langle m_{X}^{l}\right\rangle\right|_{b}\right)=\underbrace{\rho\left(\left.n_{X}^{k}\right|_{a},\left.m_{X}^{l}\right|_{a}\right)}_{\begin{array}{c}
\text { corr. of } n_{X}^{k} \text { and } m_{X}^{l} \\
\text { with same } p_{\ell, \mathrm{BRF}} \text { cut }
\end{array}} \cdot \underbrace{\rho\left(\left.\left\langle m_{X}^{l}\right\rangle\right|_{a},\left.\left\langle m_{X}^{l}\right\rangle\right|_{b}\right)}_{\begin{array}{c}
\text { corr. of moments } \\
\text { with diff. } p_{\ell, \mathrm{BRF}} \text { cut }
\end{array}} .
$$

Tables of all correlation coefficients determined with this approximation and needed for the fit are given in the appendix $\mathrm{C}$.

\subsection{Results}

All previous fits of $\mathrm{HQE}$ predictions to measured moments used moments of the leptonenergy spectrum and the hadronic-mass spectrum in decays $B \rightarrow X_{c} \ell v$ and moments of the photon-energy spectrum in decays $B \rightarrow X_{s} \gamma$. Either global fits to results from different experiments were performed, or only the data from one single experiment was used. The presented analysis is based on the results presented in [61], where only data measured by the $B A B A R$ experiment was fitted. A fit is performed replacing the hadronic mass moments by the moments of the $n_{X}^{2}$ distribution measured in this thesis up to dimension $\mathrm{GeV}^{6}$. The impact of this replacement is studied performing several fits to different sets of measured moments, combining hadronic-mass and $n_{X}^{2}$ moments. The final fit procedure is verified using Toy MC studies and the obtained results are compared to previous determinations.

\subsubsection{Experimental Input}

Selecting input moments for the fit from the large amount of available measurements is necessary due to the large correlations among the single data points. In $[35,36,61]$, fits are performed on a subset of available moments measurements with correlations below $95 \%$ to ensure the invertibility of the covariance matrix. Since the omitted measurements are characterized by high correlations to the considered ones, they do not contribute significant additional information and the overall sensitivity of the results is not affected. The final results presented in this thesis are based on the following set of moments measurements, 31 in total:

- The used lepton-energy moments are measured by BABAR [33]. The partial branching fraction $\mathcal{B}_{p_{\ell, \mathrm{BRF} \text {,min }}}$ measured for $p_{\ell, \mathrm{BRF}} \geq 0.6,1.0,1.5 \mathrm{GeV} / c$ and the moments $\left\langle E_{\ell}\right\rangle$ measured for $p_{\ell, \mathrm{BRF}} \geq 0.6,0.8,1.0,1.2,1.5 \mathrm{GeV} / c$ are used. The central leptonenergy moments $\left\langle\left(E_{\ell}-\left\langle E_{\ell}\right\rangle\right)^{2}\right\rangle$ are fitted at the minimal lepton momentum $p_{\ell, \mathrm{BRF}} \geq$ $0.6,1.0,1.5 \mathrm{GeV} / c$ and $\left\langle\left(E_{\ell}-\left\langle E_{\ell}\right\rangle\right)^{3}\right\rangle$ at $p_{\ell, \mathrm{BRF}} \geq 0.8,1.2 \mathrm{GeV} / c$. These moments are the same which are fitted in [61].

- Hadronic moments of the $n_{X}^{2}$ distribution are used as presented in this thesis. We select the following subset for the fit: $\left\langle n_{X}^{2}\right\rangle$ for $p_{\ell, \mathrm{BRF}} \geq 0.9,1.1,1.3,1.5 \mathrm{GeV} / c$, $\left\langle\left(n_{X}^{2}-\left\langle n_{X}^{2}\right\rangle\right)^{2}\right\rangle$ for $p_{\ell, \mathrm{BRF}} \geq 0.8,1.0,1.2,1.4 \mathrm{GeV} / c$, and $\left\langle\left(n_{X}^{2}-\left\langle n_{X}^{2}\right\rangle\right)^{3}\right\rangle$ for $p_{\ell, \mathrm{BRF}} \geq$ $0.8,1.0,1.2,1.4 \mathrm{GeV} / c$. Compared to [61], we replaced moments $\left\langle m_{X}^{2 / 4}\right\rangle$ by moments $\left\langle n_{X}^{2 / 4}\right\rangle$ and added moments of dimension $\mathrm{GeV}^{6}$. 
- Photon-energy moments measured in decays $B \rightarrow X_{s} \gamma$ are taken from [105] and [106]: $\left\langle E_{\gamma}\right\rangle$ for the minimal photon energy $E_{\gamma} \geq 1.9,2.0 \mathrm{GeV}$ and $\left\langle\left(E_{\gamma}-\left\langle E_{\gamma}\right\rangle\right)^{2}\right\rangle$ for $E_{\gamma} \geq 1.9 \mathrm{GeV}$. These moments are the same which are fitted in [61].

New calculations have become available since summer 2007 [107] that include a leptonmomentum dependence of the perturbative corrections for the moments of dimension $\mathrm{GeV}^{6}$. This dependence has already been available before for the moments of dimension $\mathrm{GeV}^{2}$ and $\mathrm{GeV}^{4}$. Due to the lack of these momentum-dependent corrections, the moments of dimension $\mathrm{GeV}^{6}$ could not be used in previous fits as they were not described properly by theory. We now include the measured moments $\left\langle\left(n_{X}^{2}-\left\langle n_{X}^{2}\right\rangle\right)^{3}\right\rangle$ in our investigation.

\subsubsection{Fit Results}

A comparison of the fit results for the hadronic-mass and the moments of the $n_{X}^{2}$ distribution with the measured moments is shown in figure 8.1. The mass moments are not included in the fit and thus provide an unbiased comparison with the fitted HQE prediction. We find an overall good agreement with $\chi^{2}=10.8$ for 24 degrees of freedom. The predictions for the non-central moments $\left\langle m_{X}^{k}\right\rangle(k=1,2,3)$ are below the measured moments but are still fully compatible. The measured moments continue to decrease with increasing $p_{\ell, \mathrm{BRF} \text {,min }}$ and extend beyond theoretical predictions available for $p_{\ell, \mathrm{BRF}, \min } \leq 1.5 \mathrm{GeV} / c$.

Comparing the measured moments $\left\langle n_{X}^{2}\right\rangle,\left\langle\left(n_{X}^{2}-\left\langle n_{X}^{2}\right\rangle\right)^{2}\right\rangle$ and $\left\langle\left(n_{X}^{2}-\left\langle n_{X}^{2}\right\rangle\right)^{3}\right\rangle$ with predictions resulting from the presented fit, a good agreement is found. The moments $\left\langle n_{X}^{2}\right\rangle$ are as well predicted below the measured moments.

The comparison of the measured lepton-energy moments with the fitted predictions is shown in figure 8.2. It shows a good agreement. Also the measured photon-energy moments agree well with the HQE prediction, as shown in figure 8.3.

The fit results for the standard model and HQE parameters are summarized in table 8.1. We find as results $\left|V_{c b}\right|=(41.65 \pm 0.82) \cdot 10^{-3}$ and $m_{b}=(4.570 \pm 0.055) \mathrm{GeV} / c^{2}$. The presented determination is much more sensitive to the mass difference $m_{b}-m_{c}$ than to the charm quark mass itself, which results as $m_{c}=(1.100 \pm 0.080) \mathrm{GeV} / c^{2}$. Both masses have a large correlation of $95 \%$. Therefore, we give in addition the result $m_{b}-m_{c}=$ $(3.470 \pm 0.033) \mathrm{GeV} / c^{2}$. The inclusion of the moments $\left\langle n_{X}^{6}\right\rangle$ decreases the uncertainty on the HQE parameters $\mu_{\pi}^{2}$ and $\rho_{D}^{3}$ compared to fits without them (cf. [61]). We obtain as results $\mu_{\pi}^{2}=(0.436 \pm 0.058) \mathrm{GeV}^{2}$ and $\rho_{D}^{3}=(0.179 \pm 0.034) \mathrm{GeV}^{3}$. All results are in good agreement with [61] and with earlier determinations which use experimental information from several experiments $[36,108]$. The uncertainties are slightly increased w. r. t. to the results from $[36,108]$ due to the limited experimental input used in this fit.

The impact of the moments $\left\langle n_{X}^{k}\right\rangle$ in the HQE fit is studied in section 8.5.3. We present systematic Toy MC studies concerning the fit results in subsection 8.5.4 and a detailed comparison of the fit results with previous determinations in subsection 8.5.5. 

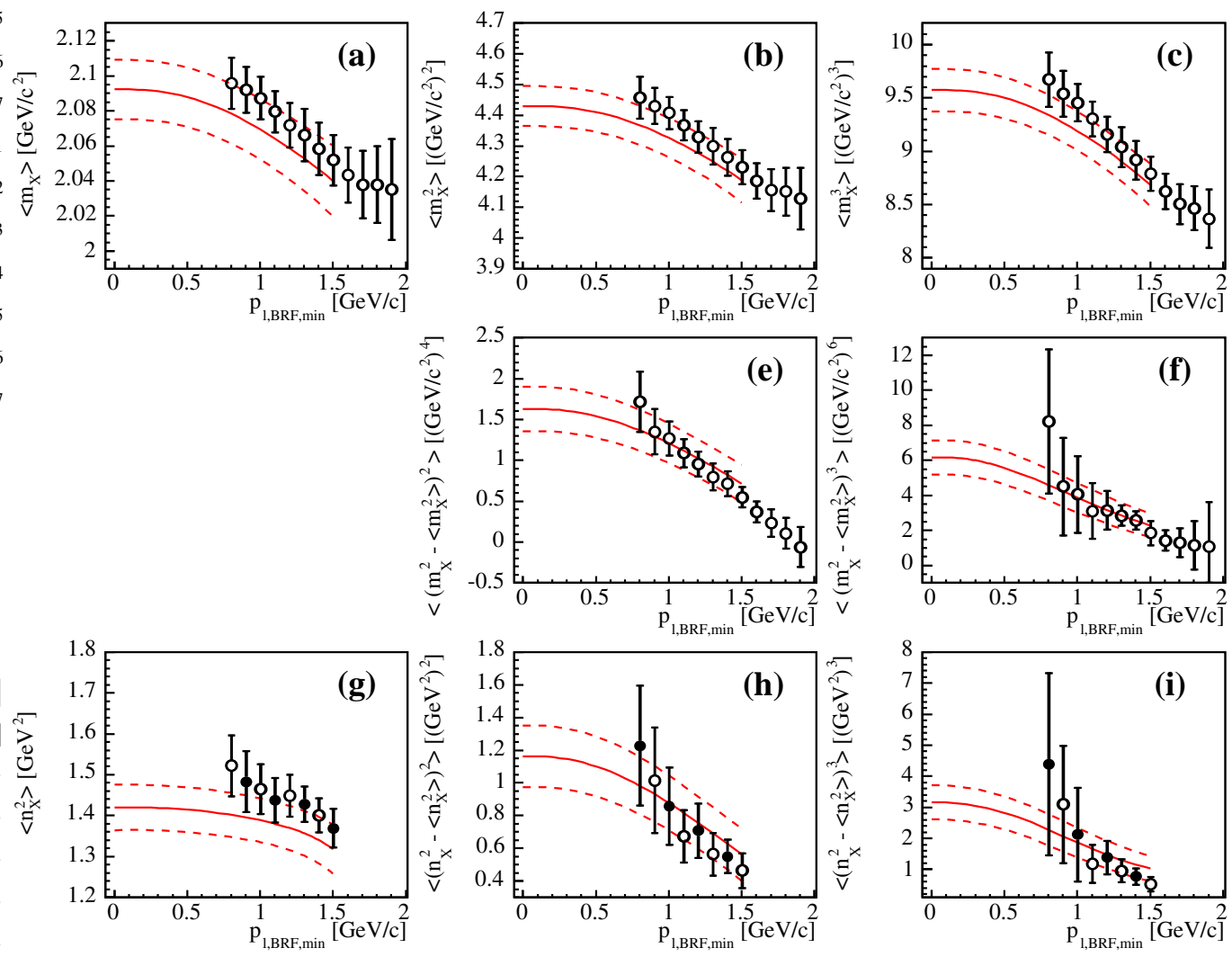

Figure 8.1: The measured hadronic-mass ((a)-(f)) and $n_{X}^{2}$ moments $((\mathrm{g})-(\mathrm{i}))(\bullet / \circ)$, as a function of the minimal lepton momentum $p_{\ell, \mathrm{BRF} \text {,min }}$ compared with the result of the simultaneous fit (solid line). The solid data points $(\bullet)$ mark the measurements included in the fit. The vertical bars indicate the experimental errors. The dashed lines correspond to the total fit uncertainty as obtained by converting the fit errors of each individual HQE parameter into an error for the individual moment. 

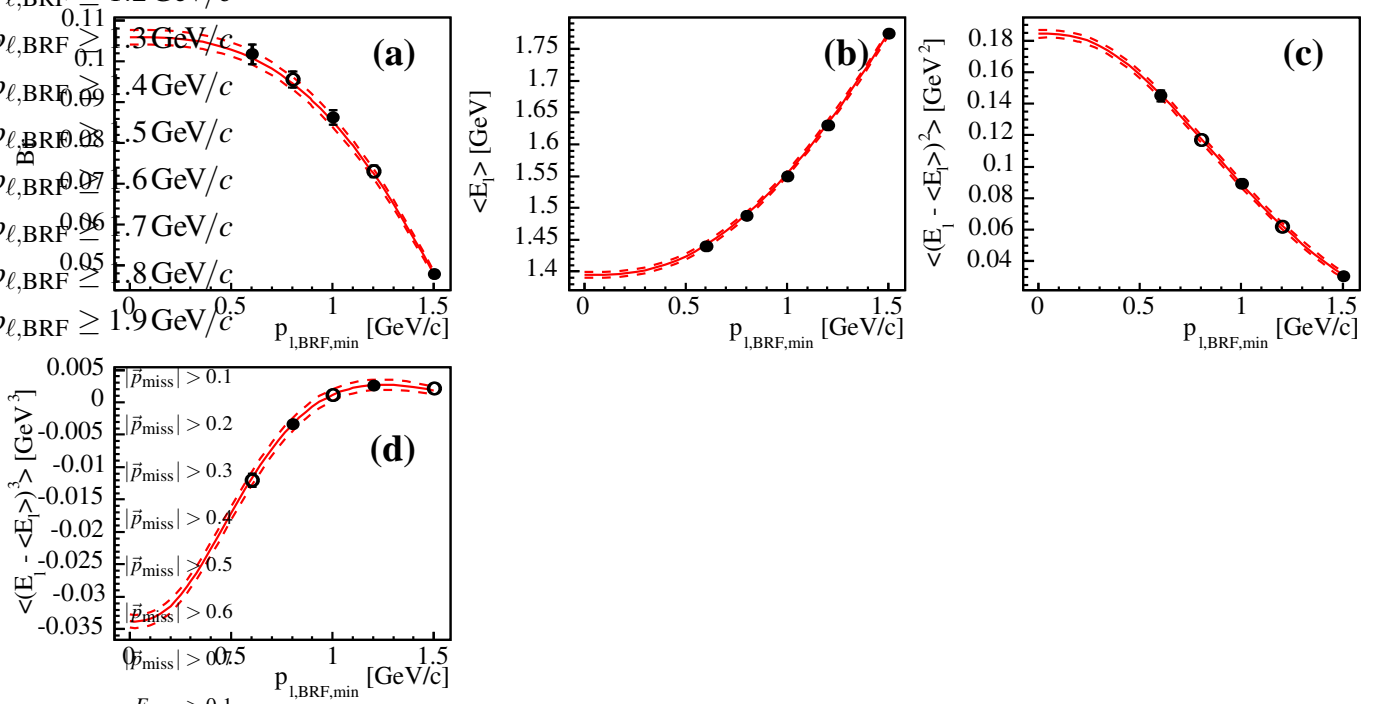

Figure 8.2: The measured lepton-energy moments $(\bullet / \circ)$, as a function of the minimal lepton momentum $p_{\ell, \mathrm{BRF}, \mathrm{min}}$ compared with the result of the simultaneous fit (solid line). The solid data points $(\bullet)$ mark the measurements included in the fit. The vertical bars indicate the experimental errors. The dashed lines correspond to the total fit uncertainty as obtained by converting the fit errors of each individual HQE parameter into an error for the individual moment.
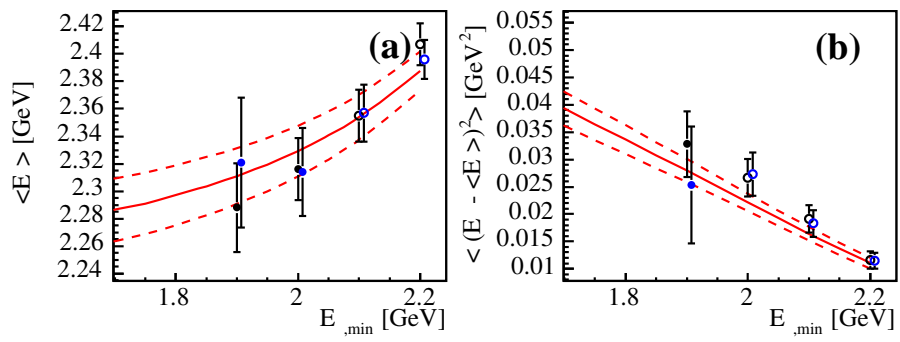

Figure 8.3: The measured photon-energy moments $(\bullet / \circ$ [106], $\bullet$ [105]) as a function of the minimal photon energy compared with the result of the simultaneous fit (solid line). The solid data points $(\bullet)$ mark the measurements included in the fit. The vertical bars indicate the experimental errors. The dashed lines correspond to the total fit uncertainty as obtained by converting the fit errors of each individual HQE parameter into an error for the individual moment. 
Table 8.1: Fit results with experimental and theoretical uncertainties. For $\left|V_{c b}\right|$ we take an additional theoretical uncertainty of $1.4 \%$ from the uncertainty in the expansion of $\Gamma_{\mathrm{SL}}$ into account. The experimental and theoretical contribution to the uncertainty of each parameter is evaluated by Toy MC studies which use only the experimental or the theoretical covariance matrix, respectively. Correlations coefficients for all parameters are summarized below the results. The parameters $m_{b}$ and $m_{c}$ are highly correlated. For the quark mass difference we find $m_{b}-m_{c}=(3.470 \pm 0.033) \mathrm{GeV} / c^{2}$.

\begin{tabular}{lrrrrrrrr}
\hline & $\begin{array}{l}\left|V_{c b}\right| \\
\times 10^{3}\end{array}$ & $\begin{array}{r}m_{b} \\
{\left[\mathrm{GeV} / c^{2}\right]}\end{array}$ & $\begin{array}{r}m_{c} \\
{\left[\mathrm{GeV} / c^{2}\right]}\end{array}$ & $\begin{array}{r}\mathcal{B} \\
{[\%]}\end{array}$ & $\begin{array}{r}\mu_{\pi}^{2} \\
{\left[\mathrm{GeV}^{2}\right]}\end{array}$ & $\begin{array}{r}\mu_{G}^{2} \\
{\left[\mathrm{GeV}^{2}\right]}\end{array}$ & $\begin{array}{r}\rho_{D}^{3} \\
{\left[\mathrm{GeV}^{3}\right]}\end{array}$ & $\begin{array}{r}\rho_{\mathrm{LS}}^{3} \\
{\left[\mathrm{GeV}^{3}\right]}\end{array}$ \\
\hline Results & 41.65 & 4.570 & 1.100 & 10.590 & 0.436 & 0.309 & 0.179 & -0.168 \\
$\Delta_{\text {exp }}$ & 0.43 & 0.033 & 0.047 & 0.172 & 0.023 & 0.040 & 0.012 & 0.073 \\
$\Delta_{\text {theo }}$ & 0.40 & 0.043 & 0.064 & 0.042 & 0.054 & 0.045 & 0.032 & 0.055 \\
$\Delta_{\Gamma_{\mathrm{SL}}}$ & 0.58 & & & & & & & \\
$\Delta_{\text {tot }}$ & 0.82 & 0.055 & 0.080 & 0.177 & 0.058 & 0.061 & 0.034 & 0.092 \\
\hline$\left|V_{c b}\right|$ & 1.00 & -0.42 & -0.22 & 0.72 & 0.29 & -0.42 & 0.18 & 0.14 \\
$m_{b}$ & & 1.00 & 0.95 & 0.08 & -0.50 & 0.15 & -0.12 & -0.19 \\
$m_{c}$ & & & 1.00 & 0.16 & -0.56 & -0.11 & -0.21 & -0.09 \\
$\mathcal{B}$ & & & & 1.00 & 0.09 & -0.10 & 0.02 & -0.04 \\
$\mu_{\pi}^{2}$ & & & & & 1.00 & 0.11 & 0.56 & -0.01 \\
$\mu_{G}^{2}$ & & & & & & 1.00 & 0.23 & -0.09 \\
$\rho_{D}^{3}$ & & & & & & & 1.00 & -0.26 \\
$\rho_{\mathrm{LS}}^{3}$ & & & & & & & & 1.00 \\
\hline
\end{tabular}




\subsubsection{Studies Using Different Input Moments}

Moments of the $n_{X}^{2}$ distribution have not been used in previous HQE fits. Following discussions with theorists [7], it is not useful perform fits to both, $n_{X}^{k}$ and $m_{X}^{l}$ moments, simultaneously. Rather, it is important to see the behavior when mass moments are completely replaced by $n_{X}^{k}$ moments, as this gives insight into the accuracy of higher order corrections which are needed for the expansion of the mass moments. This has been done as final fit result in this thesis.

To study the impact of the new moments $\left\langle n_{X}^{k}\right\rangle$ in further detail, several fits using different combinations of input moments are performed. All of them are based on the fit performed in [61], which has been updated for this thesis using the new calculations provided in fall 2007 [107]. There, the following moments are fitted:

- Lepton energy moments are used as in the final fit in this thesis and listed in subsection 8.5.1.

- Hadronic-mass moments are used as presented in [61]. The following subset is selected for the fit: $\left\langle m_{X}^{2}\right\rangle$ for $p_{\ell, \mathrm{BRF}} \geq 0.9,1.1,1.3,1.5 \mathrm{GeV} / c$ and $\left\langle\left(m_{X}^{2}-\left\langle m_{X}^{2}\right\rangle\right)^{2}\right\rangle$ for $p_{\ell, \mathrm{BRF}} \geq 0.8,1.0,1.2,1.4 \mathrm{GeV} / c$.

- Photon energy moments measured in decays $B \rightarrow X_{s} \gamma$ are used as in the final fit in this thesis and listed in subsection 8.5.1.

We investigate the influence on $\left|V_{c b}\right|, m_{b}, \mu_{\pi}^{2}$, and $\rho_{D}^{3}$ with different sets of measured moments, which are always listed w. r. t. the ones used in [61]. All fits are performed including and excluding the photon-energy moments from decays $B \rightarrow X_{s} \gamma$. We investigate the following sets of input moments:

(A) Adding moments $\left\langle\mathbf{n}_{\mathbf{X}}^{2}\right\rangle$ : Due to large correlations, only two additional data points of the moments $\left\langle n_{X}^{2}\right\rangle$ are added to the measurements listed above. We add the moments measured at $p_{\ell, \mathrm{BRF}} \geq 0.8 \mathrm{GeV} / c$ and $p_{\ell, \mathrm{BRF}} \geq 1.0 \mathrm{GeV} / c$.

(B) Replacing moments $\left\langle\left(\mathbf{m}_{\mathbf{X}}^{2}-\left\langle\mathbf{m}_{\mathbf{X}}^{2}\right\rangle\right)^{\mathbf{2}}\right\rangle$ by $\left\langle\left(\mathbf{n}_{\mathbf{X}}^{2}-\left\langle\mathbf{n}_{\mathbf{X}}^{2}\right\rangle\right)^{\mathbf{2}}\right\rangle$ : We replace all moments $\left\langle\left(m_{X}^{2}-\left\langle m_{X}^{2}\right\rangle\right)^{2}\right\rangle$ listed above by $\left\langle\left(n_{X}^{2}-\left\langle n_{X}^{2}\right\rangle\right)^{2}\right\rangle$ measured at the same cuts on the lepton momentum. All other moments are equal to [61], especially we exclude the moments $\left\langle n_{X}^{2}\right\rangle$ listed in (A).

(C) Adding moments $\left\langle\left(\mathbf{n}_{\mathbf{X}}^{\mathbf{2}}-\left\langle\mathbf{n}_{\mathbf{X}}^{\mathbf{2}}\right\rangle\right)^{\mathbf{3}}\right\rangle$ : We add moments $\left\langle\left(n_{X}^{2}-\left\langle n_{X}^{2}\right\rangle\right)^{3}\right\rangle$ measured at $p_{\ell, \mathrm{BRF}} \geq 0.9,1.1,1.3,1.5 \mathrm{GeV} / c$ to the set of moments fitted in [61].

(D) Using only $\mathbf{n}_{\mathbf{X}}^{\mathbf{k}}$ Moments : This set of moments corresponds to the final experimental input already listed in subsection 8.5.1.

Comparison plots for the results for $\left|V_{c b}\right|$ and $m_{b}$ can be found in figure 8.4. All values for $\left|V_{c b}\right|$ are compatible for all performed fits and also the uncertainty does not vary significantly. Excluding the moments from decays $B \rightarrow X_{s} \gamma$ yields a larger variation of the extracted values and uncertainties. These are, however, still fully compatible with each other. A similar behavior is found for the extracted value of $m_{b}$. While the values and uncertainties do not vary in the fits including the photon-energy moments, the uncertainty 
(D) use $\left\langle n_{X}^{2}\right\rangle,\left\langle\left(n_{X}^{2}-\left\langle n_{X}^{2}\right\rangle\right)^{2}\right\rangle,\left\langle\left(n_{X}^{2}-\left\langle n_{X}^{2}\right\rangle\right)^{3}\right\rangle$
(C) add $\left\langle\left(n_{X}^{2}-\left\langle n_{X}^{2}\right\rangle\right)^{3}\right\rangle$
(B) repl. $\left\langle\left(m_{X}^{2}-\left\langle m_{X}^{2}\right\rangle\right)^{2}\right\rangle$ by $\left\langle\left(n_{X}^{2}-\left\langle n_{X}^{2}\right\rangle\right)^{2}\right\rangle$

(A) add $\left\langle n_{X}^{2}\right\rangle$

Updated results from [61]

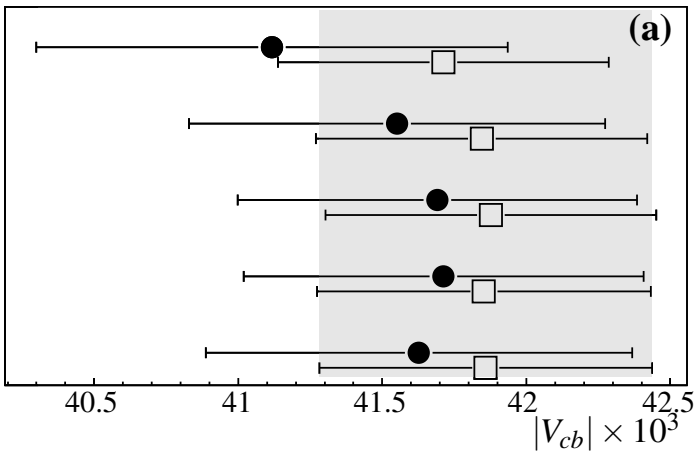

(D) use $\left\langle n_{X}^{2}\right\rangle,\left\langle\left(n_{X}^{2}-\left\langle n_{X}^{2}\right\rangle\right)^{2}\right\rangle,\left\langle\left(n_{X}^{2}-\left\langle n_{X}^{2}\right\rangle\right)^{3}\right\rangle$

(C) add $\left\langle\left(n_{X}^{2}-\left\langle n_{X}^{2}\right\rangle\right)^{3}\right\rangle$

(B) repl. $\left\langle\left(m_{X}^{2}-\left\langle m_{X}^{2}\right\rangle\right)^{2}\right\rangle$ by $\left\langle\left(n_{X}^{2}-\left\langle n_{X}^{2}\right\rangle\right)^{2}\right\rangle$

(A) add $\left\langle n_{X}^{2}\right\rangle$

Updated results from [61]

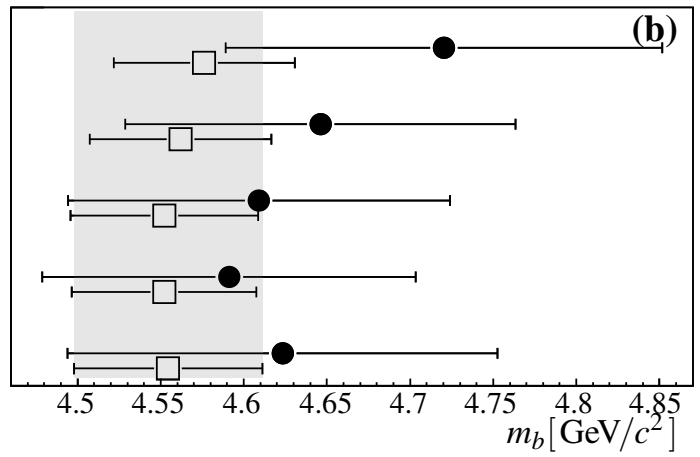

Figure 8.4: Comparison of results for $\left|V_{c b}\right|$ (a) and $m_{b}$ (b) for the different performed fits discussed above ((A)-(D)). Fits are performed including photon-energy moments from decays $B \rightarrow X_{s} \gamma(\square)$, and excluding them (•). For $\left|V_{c b}\right|$, the additional uncertainty of $1.4 \%$ from $\Gamma_{\mathrm{SL}}$ is not plotted.

gets larger by a factor of three when these moments are excluded from the fit. All values found by the various fits are compatible with each other.

In figure 8.5 similar comparison plots are shown for the extracted values of $\mu_{\pi}^{2}$ and $\rho_{D}^{3}$. The inclusion of the moments $\left\langle n_{X}^{2}\right\rangle$ (A) results in a decreased uncertainty on both parameters. An even larger effect is seen when the moments $\left\langle\left(n_{X}^{2}-\left\langle n_{X}^{2}\right\rangle\right)^{3}\right\rangle$ are included in the fit (D). The $\Delta \chi^{2}=1$ contours in the $\mu_{\pi}^{2}-m_{b},\left|V_{c b}\right|-m_{b}$, and $\mu_{\pi}^{2}-\rho_{D}^{3}$ planes are shown in figure 8.6 for the fit (D) compared to the results from [61]. The smaller ellipses also indicate the higher sensitivity of the moments $\left\langle\left(n_{X}^{2}-\left\langle n_{X}^{2}\right\rangle\right)^{3}\right\rangle$ to $\mu_{\pi}^{2}$ and $\rho_{D}^{3}$.

\subsubsection{Systematic Checks of the Fit Results}

We perform several tests to check the validity of the final fit result. The general procedure for the first so-called Toy MC test is the following:

- Taking the set of HQE parameters resulting from the final fit and presented in table 8.1, we calculate predictions for the moments included in the fit.

- These moments are smeared with the used covariance matrix, applying random factors generated according to a Gaussian distribution. 


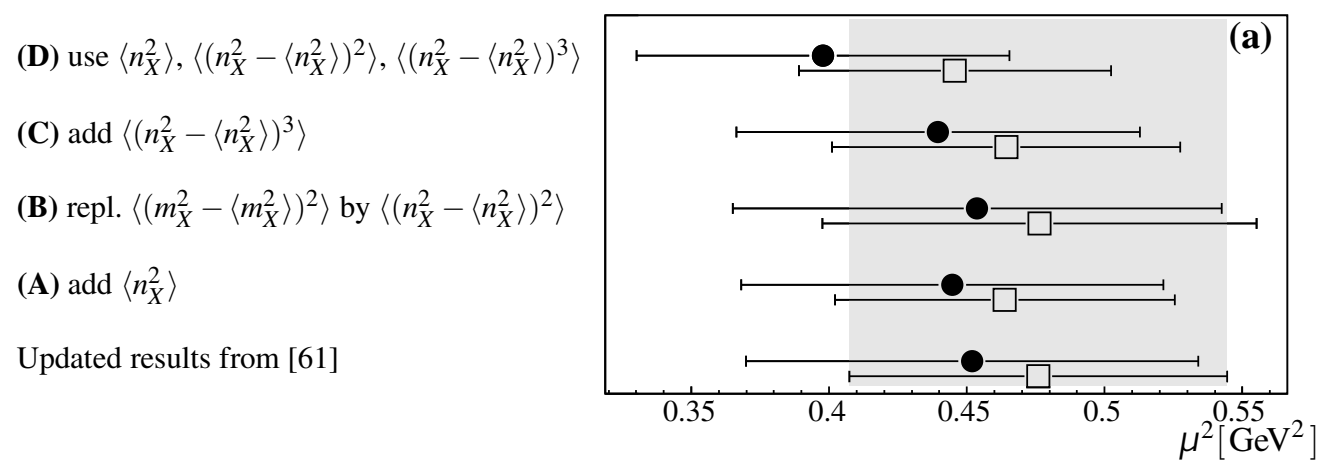

(D) use $\left\langle n_{X}^{2}\right\rangle,\left\langle\left(n_{X}^{2}-\left\langle n_{X}^{2}\right\rangle\right)^{2}\right\rangle,\left\langle\left(n_{X}^{2}-\left\langle n_{X}^{2}\right\rangle\right)^{3}\right\rangle$

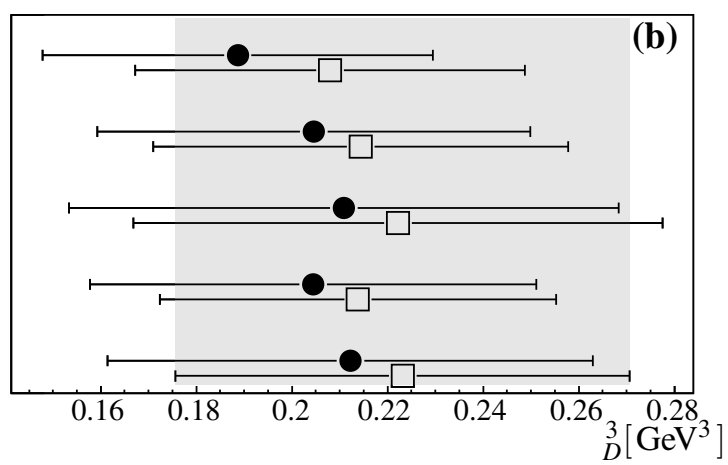

(C) add $\left\langle\left(n_{X}^{2}-\left\langle n_{X}^{2}\right\rangle\right)^{3}\right\rangle$

(B) repl. $\left\langle\left(m_{X}^{2}-\left\langle m_{X}^{2}\right\rangle\right)^{2}\right\rangle$ by $\left\langle\left(n_{X}^{2}-\left\langle n_{X}^{2}\right\rangle\right)^{2}\right\rangle$

(A) $\operatorname{add}\left\langle n_{X}^{2}\right\rangle$

Updated results from [61]

Figure 8.5: Comparison of results for $\mu_{\pi}^{2}$ (a) and $\rho_{D}^{3}$ (b) for the different performed fits discussed above ((A)-(D)). Fits are performed including photon-energy moments from decays $B \rightarrow X_{s} \gamma(\square)$, and excluding them $(\bullet)$.
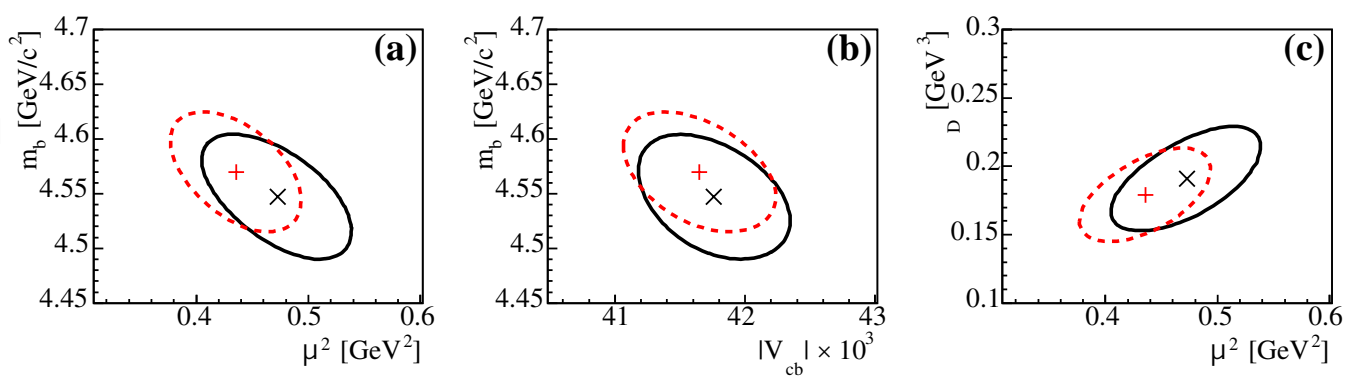

Figure 8.6: Comparison of two different fits in the $\mu_{\pi}^{2}-m_{b}$ (a), $\left|V_{c b}\right|-m_{b}$ (b), and $\mu_{\pi}^{2}-\rho_{D}^{3}$ (c) plane. Results from [61] ( $\times$, black solid line) are compared to the fit with only $n_{X}^{k}$ moments including moments $\left\langle\left(n_{X}^{2}-\left\langle n_{X}^{2}\right\rangle\right)^{3}\right\rangle$ (D) (+, red dashed ellipse). 

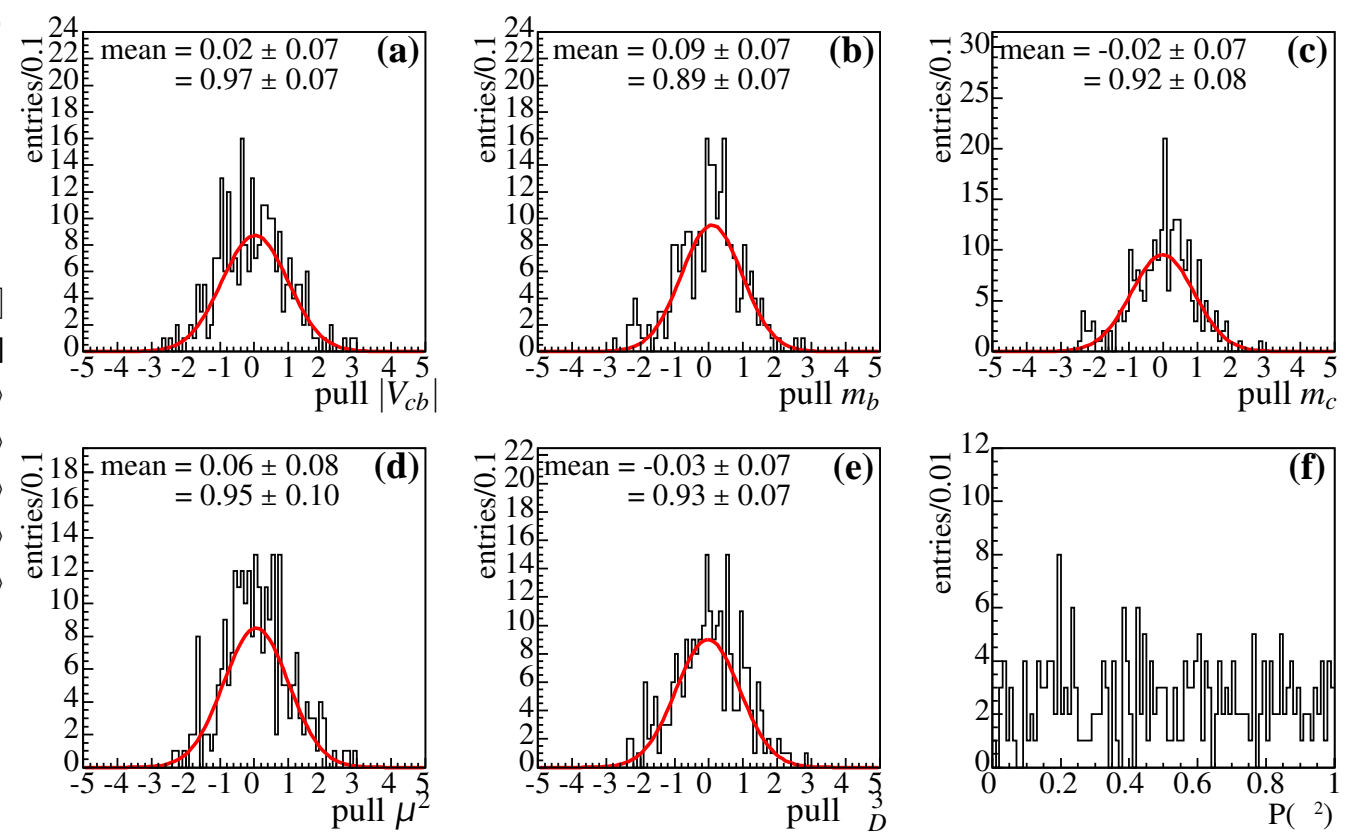

Figure 8.7: Pull distributions for the fit parameters $\left|V_{c b}\right|$ (a), $m_{b}(\mathrm{~b}), m_{c}(\mathrm{c}), \mu_{\pi}^{2}(\mathrm{~d})$, and $\rho_{D}^{3}$ (e) for Toy MC studies of the HQE fit. The pull distributions are fitted with a Gaussian function (red line), the mean and standard deviation $\sigma$ of which is given in the plots. The $\mathcal{P}\left(\chi^{2}\right)$ distribution for this study is shown in plot (f).

- We perform a fit to these random set of moments and investigate the obtained results for the eight fit parameters. This procedure is repeated 250 times.

- We investigate the pull, defined for any parameter $p$ as

$$
\text { pull }=\frac{p_{\text {fit }}-p_{\text {true }}}{\sigma_{\text {fit }}}
$$

The pull distribution is for Gaussian input error distributions expected to be of Gaussian shape with mean zero and standard deviation one. Any deviations from this shape indicate a wrong error assignment or other incorrect assumptions during the fitting procedure. Obtaining the expected shapes indicate a fitting procedure without bias.

- Furthermore we compare the errors on the parameters obtained in these Toy MC studies with the ones obtained from the original fit to data.

We find Gaussian pull distributions without significant bias and with a width of one for all fit parameters as shown in figure 8.7. The distribution of the $\chi^{2}$ probability for the Toy experiments, also shown in figure 8.7 (f), is flat as expected. The uncertainties for the fit parameters given in table 8.1 lie well within the expected error distribution obtained from the Toy experiments. 


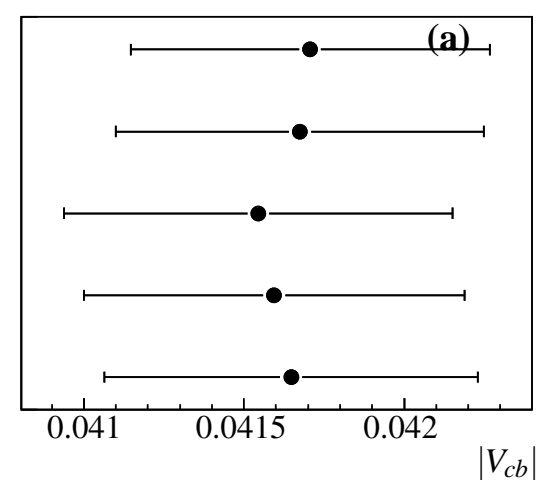

$$
\begin{aligned}
& s_{\sigma, \text { theo. }}=0.5 \\
& s_{\sigma, \text { theo. }}=0.75 \\
& s_{\sigma, \text { theo. }}=2.0 \\
& s_{\sigma, \text { theo. }}=1.5 \\
& \text { no scaling }
\end{aligned}
$$$$
V_{c b}
$$

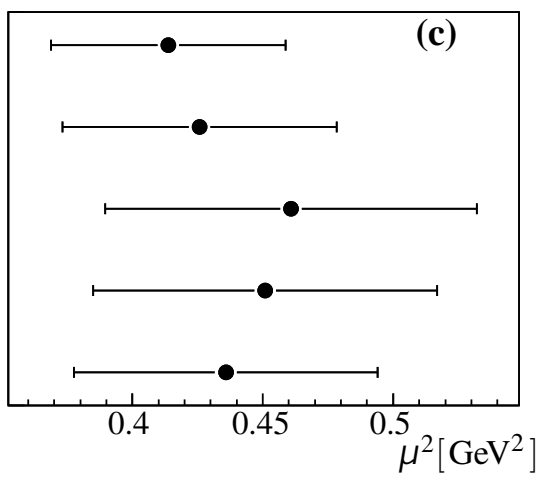

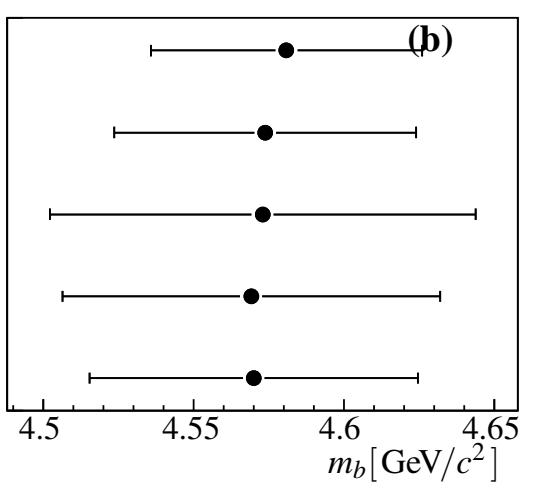

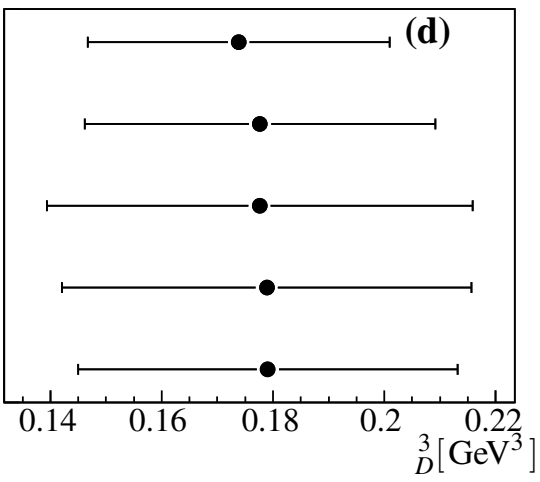

Figure 8.8: Impact of theory uncertainties of fit results. The theoretical uncertainties derived as described in section 8.3 are scaled with different scaling factors as shown in the plots. Plot (a) shows the impact on $\left|V_{c b}\right|$, (b) on $m_{b}$, (c) on $\mu_{\pi}^{2}$, and (d) on $\rho_{D}^{3}$.

Another test is performed by refitting the same set of measured moments with scaled theoretical uncertainties. The scaling factors range between 0.5 and 2.0. The results for the all fit parameters are stable within their uncertainties, as shown in figure 8.8 for the four parameters $\left|V_{c b}\right|, m_{b}, \mu_{\pi}^{2}$, and $\rho_{D}^{3}$. While the resulting uncertainty on $\left|V_{c b}\right|$ does not vary much with the theoretical uncertainties on the moment expansion, the uncertainties on the other parameters increase (decrease) with larger (smaller) theoretical uncertainties used in the fit.

\subsubsection{Comparison of Results with Previous Determinations}

Various previous results for the parameters extracted in this thesis exist. Some are even extracted in similar ways, that is from HQE fits to moments measurements. The uncertainties on the fit parameters in these HQE fits depend on the fitted input moments. Thus, uncertainties from global fits cannot directly be compared to the ones obtained in this analysis. Some parameters, such as $\left|V_{c b}\right|, m_{b}$ or $\mu_{\pi}^{2}$ can also be extracted from different measurements, e. g. exclusive decays $B \rightarrow D^{*} \ell v$, or fits to observables in $b \rightarrow u \ell v$ decays. In this section, the results of this thesis are compared to previous determinations. 
Result for $\left|\mathbf{V}_{\mathbf{c b}}\right|$ As discussed in chapter 2, the quark mixing parameter may not only be extracted from inclusive semileptonic decays of $B$ mesons, but also from the measured spectrum $\mathrm{d} \mathcal{B} / \mathrm{d} w$ measured in exclusive decays $B \rightarrow D^{*} \ell v$. Using this method, $\left|V_{c b}\right|$ is determined in combination with a form factor $\mathcal{F}(1)$. Averaging all exclusive measurements yields in summer 2007 the value $\left|V_{c b}\right| \cdot \mathcal{F}(1)=(35.89 \pm 0.56) \cdot 10^{-3}$ [24]. With a value of $\mathcal{F}(1)=0.919 \pm 0.033$ calculated by lattice QCD [109], this value corresponds to $\left|V_{c b}\right|=$ $(39.05 \pm 1.5) \cdot 10^{-3}$.

The result with the smallest uncertainty derived from inclusive decays in the kinetic scheme is obtained from a combined fit to moments measurements of many experiments [36]. An inclusion of hadronic-mass moments measured by the Belle collaboration [5] in this global fit in summer 2007 yields a value of $\left|V_{c b}\right|=(41.91 \pm 0.68) \cdot 10^{-3}$, which is in perfect agreement with our result $\left|V_{c b}\right|=(41.65 \pm 0.82) \cdot 10^{-3}$. A fit performed in the $1 \mathrm{~S}$ scheme [108] also yields compatible results.

The value of $\left|V_{c b}\right|$ extracted from inclusive decays is in agreement with the one extracted from exclusive decays on the $1.6 \sigma$ level. This discrepancy is not understood until now.

Result for the Semileptonic Rate The Heavy Flavor Averaging Group determines the total semileptonic rate for an admixture of neutral and charged $B$ mesons from different measurements. The full branching fraction is extracted as $\mathcal{B}\left(B^{+} / B^{0} \rightarrow X \ell v\right)=(10.75 \pm$ $0.16) \%$ [24]. Our result $\mathcal{B}\left(B \rightarrow X_{c} \ell v\right)=(10.59 \pm 0.18) \%$ is in good agreement with this number, taking the ratio $\left|V_{u b}\right|^{2} /\left|V_{c b}\right|^{2} \approx 0.01$ and phase space factors into account to extrapolate our result to the total semileptonic rate.

Results for the Bottom Quark Mass and the Heavy Quark Parameter $\boldsymbol{\mu}_{\boldsymbol{\pi}}^{2}$ The $b$ quark mass in the kinetic scheme can also be extracted in similar HQE fits of mass moments in decays $B \rightarrow X_{u} \ell v$ and, as already pointed out, from photon-energy moments in decays $B \rightarrow X_{s} \gamma$. Due to the smaller branching fractions of the decays $B \rightarrow X_{u} \ell v$ by a factor $10^{-2}$, the measured moments have much larger uncertainties, resulting in larger uncertainties of $m_{b}$ and $\mu_{\pi}^{2}$. A fit performed by the BABAR collaboration and presented in in [110] yields results for $m_{b}$ and $\mu_{\pi}^{2}$ as shown in figure 8.9. There, also the result from decays $B \rightarrow X_{s} \gamma$ alone, as presented in [106], is shown. Furthermore, the final fit presented in this thesis has been repeated without the photon-energy moments and the results are shown as well.

The results of all four determinations are in agreement. However, fits excluding the photon-energy moments result in a larger value of $m_{b}$. The fit to photon-energy moments alone yield a larger value of $\mu_{\pi}^{2}$. Our result for the HQE parameter $\mu_{\pi}^{2}=(0.436 \pm$ $0.058) \mathrm{GeV}^{2}$ is in good agreement with the one obtained in [36], which is $\mu_{\pi}^{2}=(0.401 \pm$ $0.040) \mathrm{GeV}^{2}$, and with the one presented in [61], $\mu_{\pi}^{2}=(0.471 \pm 0.070) \mathrm{GeV}^{2}$. Comparing our result to the latter, shows a decreased uncertainty, achieved by the inclusion of the moments $\left\langle n_{X}^{6}\right\rangle$. Comparing our result $m_{b}=(4.570 \pm 0.055) \mathrm{GeV} / c^{2}$ to the one obtained in [36], which is $m_{b}=(4.590 \pm 0.039) \mathrm{GeV} / c^{2}$, we find a good agreement.

As pointed out before, quark masses can be determined in different renormalization schemes. We translate our result in the kinetic scheme to the $\overline{\mathrm{MS}}$ scheme, using a calculation up to order $\alpha_{s}^{2}[32,107]$. The formula is given in eq. (D.1) in the appendix. We obtain $m_{b}\left(m_{b}\right)=(4.205 \pm 0.048 \pm 0.015) \mathrm{GeV} / c^{2}$, where the first error is the translated uncertainty from the kinetic scheme and the second is an estimate for the uncer- 


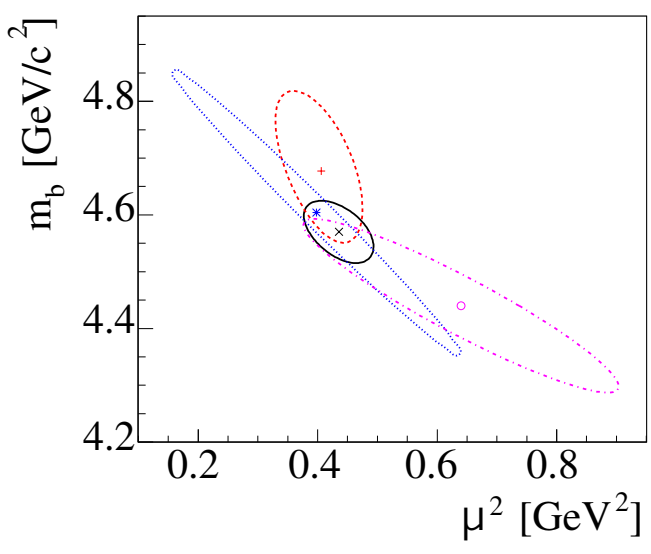

Figure 8.9: Comparison of four different fits in the $\mu_{\pi}^{2}-m_{b}$ plane. The $\Delta \chi^{2}=1$ contours in this plane are plotted. Results from this thesis $(\times$, black solid line $)$ are compared to the same fit excluding the photonenergy moments ( + , red dashed ellipse), to a fit to hadronic-mass moments in decays $B \rightarrow X_{u} \ell v$ (*,blue dotted ellipse) [110], and to the results from decays $B \rightarrow X_{s} \gamma$ alone [106] ( $\circ$, magenta dash-dotted ellipse).

tainty of the transformation itself. The value is in good agreement with the world average $m_{b}\left(m_{b}\right)=(4.20 \pm 0.07) \mathrm{GeV} / c^{2}[42]$.

Results for the Charm Quark Mass The charm quark mass obtained in this analysis $m_{c}=(1.100 \pm 0.080) \mathrm{GeV} / c^{2}$ is determined in the kinetic scheme and can be compared to the result from [36], $m_{c}=(1.142 \pm 0.058) \mathrm{GeV} / c^{2}$. These two values are in good agreement.

To compare our result for the mass of the charm quark to the results given in [42] the result obtained in the kinetic scheme has to be translated to the $\overline{\mathrm{MS}}$ scheme. Results for $m_{c}$ are obtained either from similar HQE fits, from calculations of the $D_{s}$ mass, or from the $e^{+} e^{-}$cross section to hadrons, to name only a few methods. All results are averaged in [42] and result in $1.25 \pm 0.09 \mathrm{GeV} / \mathrm{c}^{2}$. Our result converted to the $\overline{\mathrm{MS}}$ scheme, using a transformation at order $\alpha_{s}^{2}[32,107]$, is $m_{c}\left(m_{c}\right)=(1.214 \pm 0.063 \pm 0.050) \mathrm{GeV} / c^{2}$, where the first error is the translated uncertainty from the kinetic scheme and the second is an estimate for the uncertainty of the transformation itself [107]. The value is compatible with the world average.

Results for the Heavy Quark Parameters $\mu_{\mathrm{G}}^{2}, \boldsymbol{\rho}_{\mathrm{D}}^{\mathbf{3}}$, and $\boldsymbol{\rho}_{\mathrm{LS}}^{\mathbf{3}}$ Heavy quark parameters have been extracted from previous HQE fits to moments measurements. As discussed above, the two parameters $\mu_{G}^{2}$ and $\rho_{\mathrm{LS}}^{3}$ have been estimated from $B-B^{*}$ mass splitting and heavy-quark sum rules to be $\mu_{G}^{2}=(0.35 \pm 0.07) \mathrm{GeV}^{2}$ and $\rho_{\mathrm{LS}}^{3}=(-0.15 \pm 0.10) \mathrm{GeV}^{3}$ [36], respectively. They are fitted using Gaussian error constraints around these values. The obtained results lie well within these error regions and the obtained uncertainties are compatible.

The HQE parameter $\rho_{D}^{3}$ is one of the parameters, to which the measured moments of the $n_{X}^{2}$ distribution are expected to be especially sensitive. Our result $\rho_{D}^{3}=(0.179 \pm$ $0.034) \mathrm{GeV}^{3}$ is in good agreement with the numbers from [36,61], which are $\rho_{D}^{3}=(0.174 \pm$ $0.024) \mathrm{GeV}^{3}$ and $\rho_{D}^{3}=(0.220 \pm 0.047) \mathrm{GeV}^{3}$, respectively. In another determination by the Belle Collaboration [111] a number of $\tilde{\rho}_{D}^{3}=0.162 \pm 0.054 \mathrm{GeV}^{3}$ is given, where the socalled on-shell parameter $\tilde{\rho}_{D}^{3}$ is defined as $\rho_{D}^{3}-0.1 \mathrm{GeV}^{3}$. This value deviates from our measurement by 1.3 standard deviations and from the $\rho_{D}^{3}$ determined in [36] by 1.5 stan- 
dard deviations assuming uncorrelated uncertainties. As the different fits use the same theoretical calculations, the correlations of the uncertainties is probably larger than zero resulting in a larger discrepancy. However, this discrepancy of the measurement presented in [111] with other determinations is not understood [112].

Summary of the Comparison In summary, all fit results presented in this thesis are in agreement with previous determinations. Uncertainties are larger than obtained from global HQE fits due to the limited experimental input. The inclusion of moments of order $\mathrm{GeV}^{6}$ lead do decreased uncertainties on the parameters $\mu_{\pi}^{2}$ and $\rho_{D}^{3}$. The inclusion or replacement of the hadronic mass moments by moments of the $n_{X}^{2}$ distribution lead to stable results concerning all fit parameters. With further insight into the theoretical expansions [7], this can be interpreted as a solid hint that higher order corrections, which are needed for the expansion of the hadronic mass moments, but not for the $n_{X}^{2}$ moments, have been estimated correctly. A significant change in the uncertainties of the HQE and SM parameters by the tests performed in this thesis would instead have indicated a somehow too naive treatment of these corrections for the mass moments. Consequently, the presented results have given an extended insight into the validity of error estimates that have to be made for a reliable extraction of SM parameters. 


\section{Summary and Conclusion}

This thesis presents the first measurement of moments of the combined hadronic mass and energy spectrum in semileptonic decays to hadronic final states containing a charm quark, $B \rightarrow X_{c} \ell v$. The moments are determined from the $n_{X}^{2}$ distribution of the hadronic system, where $n_{X}^{2}$ is defined from the invariant mass, the energy in the $B$-meson rest frame and a constant $\tilde{\Lambda}=0.65 \mathrm{GeV}$ as

$$
n_{X}^{2}=m_{X}^{2} c^{4}-2 \tilde{\Lambda} E_{X, \mathrm{BRF}}+\tilde{\Lambda}^{2} .
$$

The analysis is based on a sample of 231.6 million $e^{+} e^{-} \rightarrow \Upsilon(4 S) \rightarrow B \bar{B}$ events recorded with the $B A B A R$ experiment. We employ a technique that fully reconstructs one of the $B$ mesons in the event in several hadronic decay modes, thereby separating the decay products of both $B$ meson decays. The semileptonic decay of the second $B$ meson in the event is identified by its associated lepton.

The reconstructed momentum and energy of the hadronic system are distorted by missing particles that emerge the detector acceptance and by the resolution of the detector. These effects are studied comprehensively in a MC simulation of the $B$ meson decays and the detector response. Correction methods are implemented to get an unbiased measurement of the moments of the $n_{X}^{2}$ distribution. The remaining background contribution of about $20 \%$ is subtracted using its shape from data itself if possible and from MC simulations. This allows the measurement of the moments $\left\langle n_{X}^{k}\right\rangle$ with $k=2,4,6$ for different selection criteria on the minimal lepton momentum between $p_{\ell} \geq 0.8 \mathrm{GeV} / c$ and $p_{\ell} \geq 1.9 \mathrm{GeV} / c$ determined in the rest frame of the $B$ meson.

Various sources of systematic uncertainties of this measurement are investigated, where the main contribution arises from the impact of the reconstruction efficiency of neutral particles on the applied technique of full event reconstruction.

Moments of the hadronic $n_{X}^{2}$ distribution are predicted in the framework of a Heavy Quark Expansion performed in the kinetic scheme. Similar calculations also exist for hadronic-mass moments and for lepton-energy moments, both previously measured in the same type of $B$ meson decays, $B \rightarrow X_{c} \ell v$. Furthermore, photon-energy moments in decays $B \rightarrow X_{s} \gamma$ are predicted by the same theory.

Performing a simultaneous fit to the measured moments $\left\langle n_{X}^{k}\right\rangle$ up to order $\mathrm{GeV}^{6}$ combined with other measurements of moments of the lepton-energy spectrum in decays $B \rightarrow$ $X_{c} \ell \nu$ and moments of the photon-energy spectrum in decays $B \rightarrow X_{s} \gamma$, we determine the quark-mixing parameter $\left|V_{c b}\right|$, the bottom and charm quark masses, the semileptonic branching fraction $\mathcal{B}\left(B \rightarrow X_{c} \ell v\right)$, and four non-perturbative heavy quark parameters. Using HQE calculations in the kinetic scheme up to order $1 / m_{b}^{3}$, we find

$$
\left|V_{c b}\right|=(41.65 \pm 0.43 \pm 0.40 \pm 0.58) \cdot 10^{-3} \text { and } m_{b}=(4.570 \pm 0.033 \pm 0.043) \mathrm{GeV} / c^{2}
$$

where the first uncertainty refers to experimental contributions, the second to uncertainties in the HQE, and the third ( $\left|V_{c b}\right|$ only) to theoretical uncertainties in the calculations of the 
semileptonic decay rate $\Gamma\left(B \rightarrow X_{c} \ell v\right)$. All obtained results are consistent with previous determinations and the theoretically predicted moments are in good consistency with the measured moments. The inclusion of the moments $\left\langle n_{X}^{6}\right\rangle$ decreases the uncertainty on the HQE parameters $\mu_{\pi}^{2}$ and $\rho_{D}^{3}$ compared to fits without them,

$$
\mu_{\pi}^{2}=(0.436 \pm 0.058) \mathrm{GeV}^{2} \text { and } \rho_{D}^{3}=(0.179 \pm 0.034) \mathrm{GeV}^{3} .
$$

Results from previous fits that used hadronic-mass moments instead of the moments of the $n_{X}^{2}$ distributions are confirmed. This fact verifies assumptions needed for the treatment of higher order corrections in the expansion of the mass moments, as these corrections are strongly suppressed in the case of the $n_{X}^{2}$ moments. This is an important and successful test for the theoretical framework of Heavy Quark Expansions. 


\section{A Calibration Curves for $\mathbf{n}_{\mathbf{X}}^{4}$ and $\mathbf{n}_{\mathbf{X}}^{6}$}

In this appendix, we give the calibration curves for the moments $\left\langle n_{X}^{4}\right\rangle$ and $\left\langle n_{X}^{6}\right\rangle$. Again they are measured separately for electrons and muons. Details on the calibration procedure can be found in section 6.8.2.
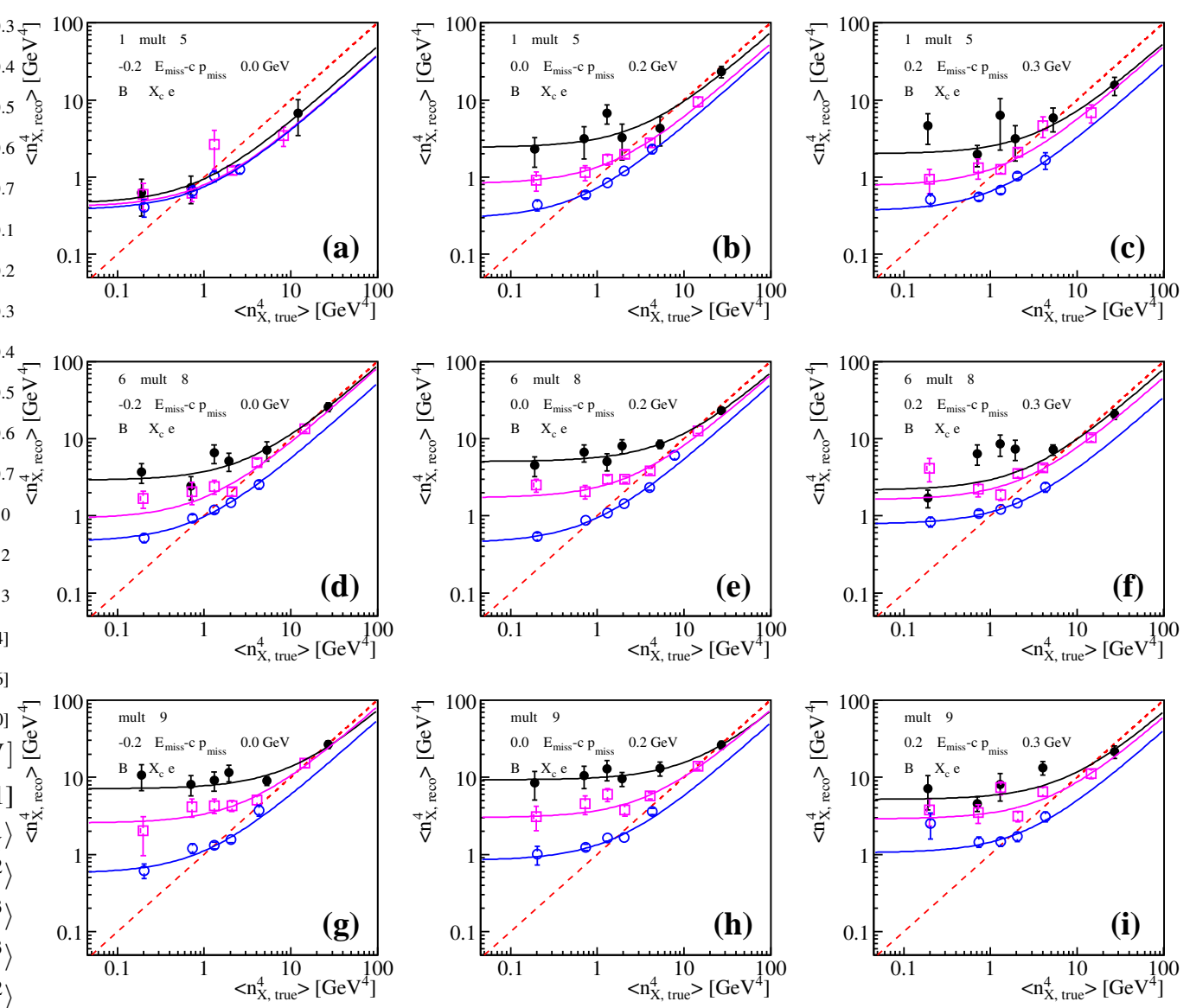

Figure A.1: Examples of calibration curves for $\left\langle n_{X}^{4}\right\rangle$ in bins of multiplicity, $E_{\text {miss }}-c\left|\vec{p}_{\text {miss }}\right|$ ((a)-(i)), and $p_{\ell, \mathrm{BRF}}$ (color code), extracted for events $B \rightarrow X_{c} e v$. Shown are the extracted $\left\langle n_{X, \text { reco }}^{4}\right\rangle$ versus $\left\langle n_{X \text {,true }}^{4}\right\rangle$ in bins of $n_{X \text { true }}^{2}$ for $0.9<p_{\ell, \mathrm{BRF}} \leq 1.0 \mathrm{GeV} / c(\bullet)$, $1.4<p_{\ell, \mathrm{BRF}} \leq 1.5 \mathrm{GeV} / c(\square)$, and $p_{\ell, \mathrm{BRF}} \geq 1.9 \mathrm{GeV} / c(\circ)$. The results of fits of linear functions are overlaid as solid lines. Reference lines with $\left\langle n_{X, \text { reco }}^{k}\right\rangle=\left\langle n_{X, \text { true }}^{k}\right\rangle$ are superimposed (dashed lines). 

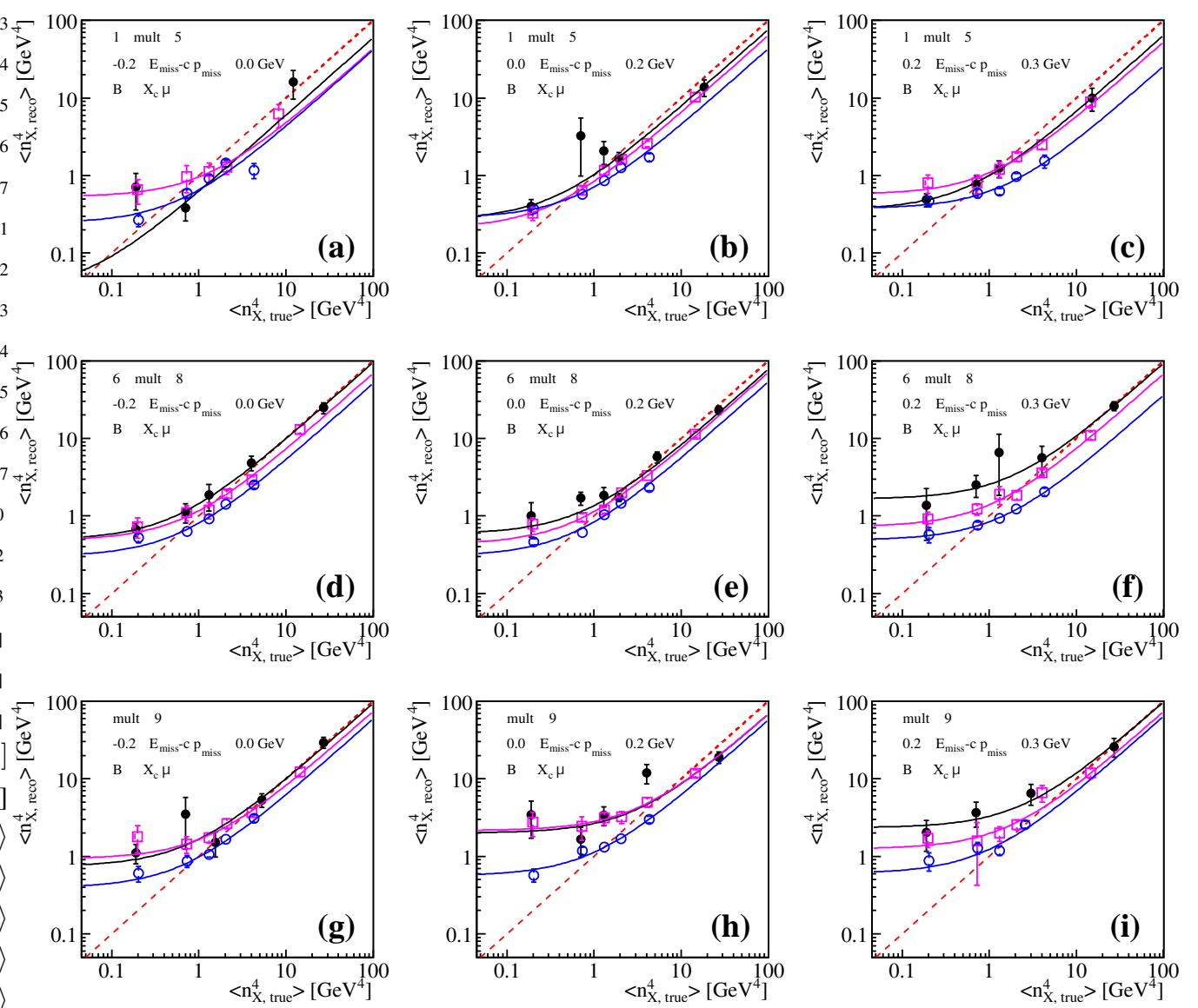

Figure A.2: Examples of calibration curves for $\left\langle n_{X}^{4}\right\rangle$ in bins of multiplicity, $E_{\text {miss }}-c\left|\vec{p}_{\text {miss }}\right|$ ((a)-(i)), and $p_{\ell, \text { BRF }}$ (color code), extracted for events $B \rightarrow X_{c} \mu v$. Shown are the extracted $\left\langle n_{X, \text { reco }}^{4}\right\rangle$ versus $\left\langle n_{X, \text { true }}^{4}\right\rangle$ in bins of $n_{X \text { true }}^{2}$ for $0.9<p_{\ell, \mathrm{BRF}} \leq 1.0 \mathrm{GeV} / c(\bullet)$, $1.4<p_{\ell, \mathrm{BRF}} \leq 1.5 \mathrm{GeV} / c(\square)$, and $p_{\ell, \mathrm{BRF}} \geq 1.9 \mathrm{GeV} / c$ (०). The results of fits of linear functions are overlaid as solid lines. Reference lines with $\left\langle n_{X, \text { reco }}^{k}\right\rangle=\left\langle n_{X, \text { true }}^{k}\right\rangle$ are superimposed (dashed lines). 

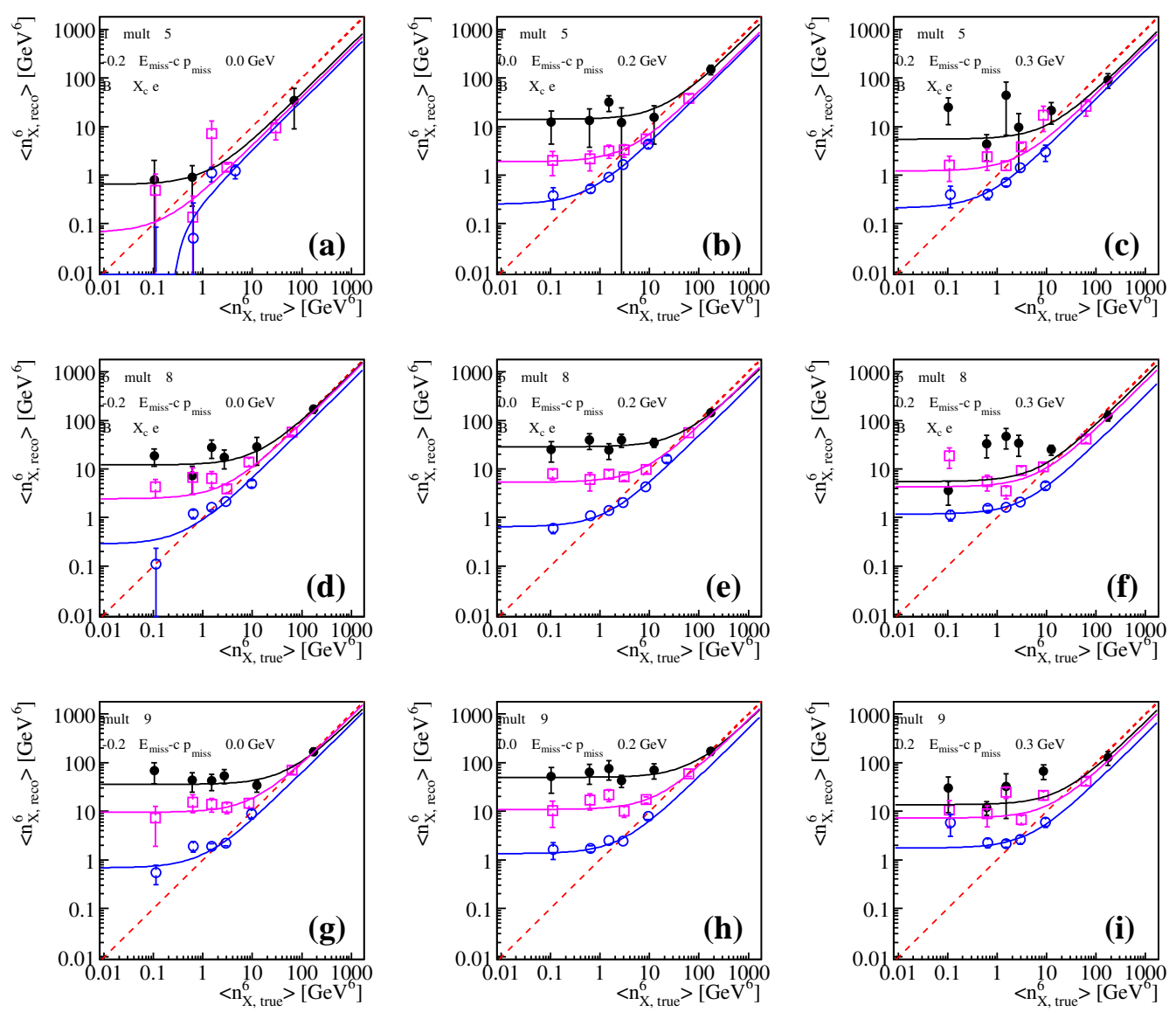

Figure A.3: Examples of calibration curves for $\left\langle n_{X}^{6}\right\rangle$ in bins of multiplicity, $E_{\text {miss }}-c\left|\vec{p}_{\text {miss }}\right|$ ((a)-(i)), and $p_{\ell, \mathrm{BRF}}$ (color code), extracted for events $B \rightarrow X_{c} e v$. Shown are the extracted $\left\langle n_{X, \text { reco }}^{6}\right\rangle$ versus $\left\langle n_{X, \text { true }}^{6}\right\rangle$ in bins of $n_{X \text { true }}^{2}$ for $0.9<p_{\ell, \mathrm{BRF}} \leq 1.0 \mathrm{GeV} / c(\bullet)$, $1.4<p_{\ell, \mathrm{BRF}} \leq 1.5 \mathrm{GeV} / c(\square)$, and $p_{\ell, \mathrm{BRF}} \geq 1.9 \mathrm{GeV} / c(\circ)$. The results of fits of linear functions are overlaid as solid lines. Reference lines with $\left\langle n_{X, \text { reco }}^{k}\right\rangle=\left\langle n_{X, \text { true }}^{k}\right\rangle$ are superimposed (dashed lines). 

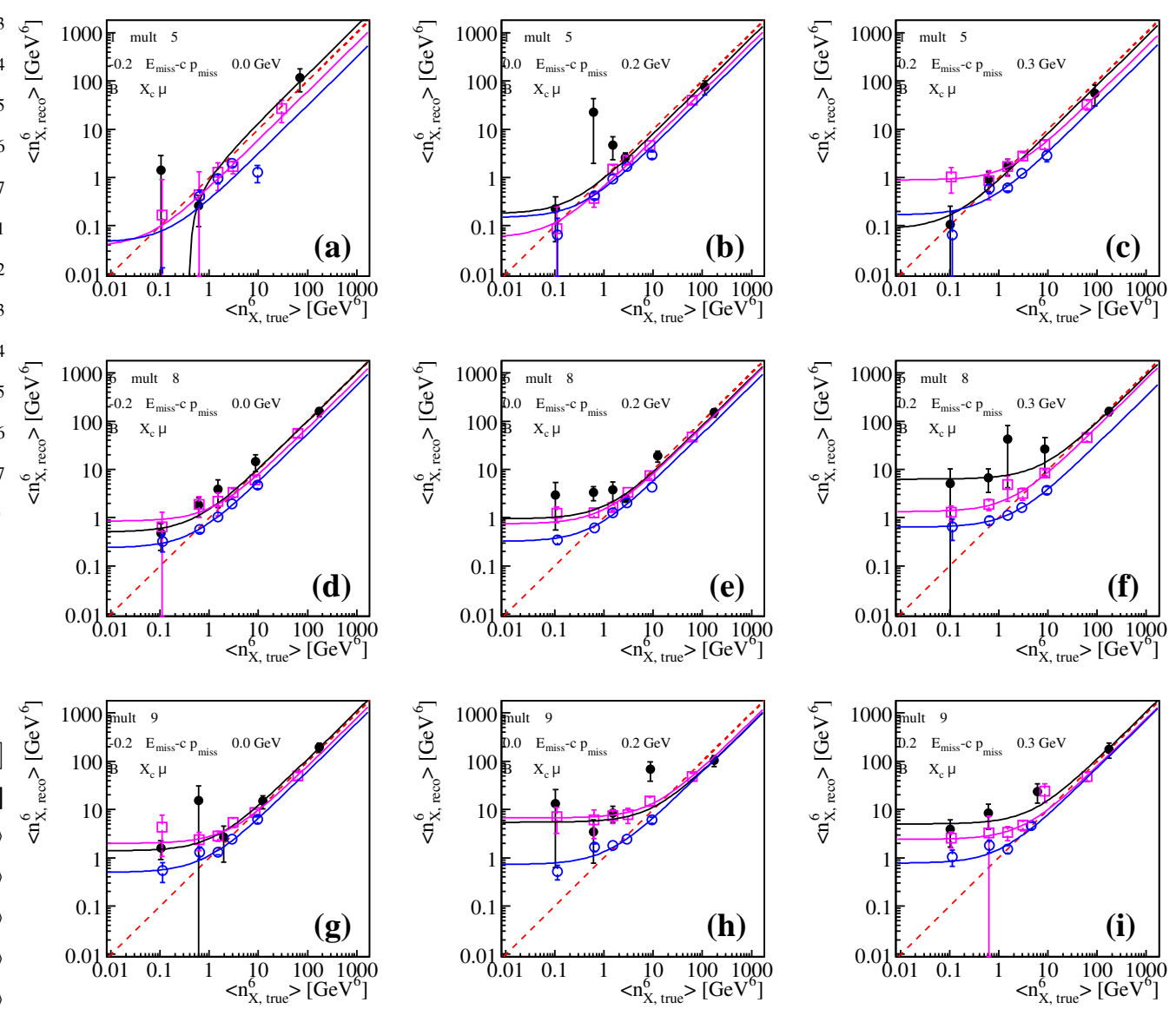

Figure A.4: Examples of calibration curves for $\left\langle n_{X}^{6}\right\rangle$ in bins of multiplicity, $E_{\text {miss }}-c\left|\vec{p}_{\text {miss }}\right|$ ((a)-(i)), and $p_{\ell, \mathrm{BRF}}$ (color code), extracted for events $B \rightarrow X_{c} \mu \nu$. Shown are the extracted $\left\langle n_{X, \text { reco }}^{6}\right\rangle$ versus $\left\langle n_{X, \text { true }}^{6}\right\rangle$ in bins of $n_{X \text { true }}^{2}$ for $0.9<p_{\ell, \mathrm{BRF}} \leq 1.0 \mathrm{GeV} / c(\bullet)$, $1.4<p_{\ell, \mathrm{BRF}} \leq 1.5 \mathrm{GeV} / c(\square)$, and $p_{\ell, \mathrm{BRF}} \geq 1.9 \mathrm{GeV} / c(\circ)$. The results of fits of linear functions are overlaid as solid lines. Reference lines with $\left\langle n_{X, \text { reco }}^{k}\right\rangle=\left\langle n_{X, \text { true }}^{k}\right\rangle$ are superimposed (dashed lines). 


\section{B Correlation Matrices for the Moments $\mathbf{n}_{X}^{2}, \mathbf{n}_{X}^{4}$, and $\mathbf{n}_{X}^{6}$}

The correlation matrices for the moments $\left\langle n_{X}^{2}\right\rangle,\left\langle n_{X}^{4}\right\rangle$, and $\left\langle n_{X}^{6}\right\rangle$ are presented in this appendix. The matrices use statistical correlations only. The formalism to determine these numbers is reported in section 6.8.4. 


\begin{tabular}{|c|c|c|c|c|c|c|c|c|c|c|c|c|c|c|c|c|c|c|c|c|c|c|c|c|c|}
\hline \multirow{2}{*}{\multicolumn{2}{|c|}{$p_{l, \mathrm{BRF}, \min }[\mathrm{GeV} / c]$}} & \multicolumn{12}{|c|}{$\left\langle n_{X}^{2}\right\rangle\left[\mathrm{GeV}^{2}\right]$} & \multicolumn{12}{|c|}{$\mid\left\langle n_{X}^{4}\right\rangle\left[\mathrm{GeV}^{4}\right]$} \\
\hline & & 0.8 & 0.9 & 1.0 & 1.1 & 1.2 & 1.3 & 1.4 & 1.5 & 1.6 & 1.7 & 1.8 & 1.9 & 0.8 & 0.9 & 1.0 & 1.1 & 1.2 & 1.3 & 1.4 & 1.5 & 1.6 & 1.7 & 1.8 & 1.9 \\
\hline \multirow[t]{12}{*}{$\left\langle n_{X}^{2}\right\rangle$} & 0.8 & 1.00 & 0.88 & 0.77 & 0.67 & 0.58 & 0.50 & 0.43 & 0.38 & 0.31 & 0.26 & 0.21 & 0.15 & $\mid 0.92$ & 0.81 & 0.69 & 0.59 & 0.50 & 0.42 & 0.37 & 0.32 & 0.26 & 0.21 & 0.17 & 0.11 \\
\hline & 0.9 & & 1.00 & 0.87 & 0.75 & 0.66 & 0.56 & 0.49 & 0.43 & 0.36 & 0.30 & 0.24 & 0.17 & 0.74 & 0.91 & 0.79 & 0.67 & 0.57 & 0.48 & 0.41 & 0.36 & 0.29 & 0.24 & 0.19 & 0.13 \\
\hline & 1.0 & & & 1.00 & 0.86 & 0.75 & 0.65 & 0.56 & 0.49 & 0.41 & 0.34 & 0.28 & 0.20 & 0.57 & 0.71 & 0.90 & 0.76 & 0.65 & 0.55 & 0.47 & 0.41 & 0.33 & 0.27 & 0.22 & 0.14 \\
\hline & 1.1 & & & & 1.00 & 0.87 & 0.75 & 0.65 & 0.57 & 0.47 & 0.40 & 0.32 & 0.23 & 0.43 & 0.53 & 0.68 & 0.88 & 0.76 & 0.64 & 0.55 & 0.47 & 0.38 & 0.32 & 0.25 & 0.17 \\
\hline & 1.2 & & & & & 1.00 & 0.86 & 0.75 & 0.66 & 0.54 & 0.46 & 0.37 & 0.27 & 0.33 & 0.41 & 0.52 & 0.68 & 0.87 & 0.73 & 0.63 & 0.54 & 0.44 & 0.36 & 0.29 & 0.19 \\
\hline & 1.3 & & & & & & 1.00 & 0.87 & 0.76 & 0.63 & 0.53 & 0.43 & 0.31 & 0.25 & 0.30 & 0.39 & 0.51 & 0.65 & 0.85 & 0.73 & 0.63 & 0.51 & 0.42 & 0.34 & 0.22 \\
\hline & 1.4 & & & & & & & 1.00 & 0.88 & 0.72 & 0.61 & 0.49 & 0.35 & 0.18 & 0.23 & 0.29 & 0.38 & 0.48 & 0.63 & 0.84 & 0.73 & 0.59 & 0.49 & 0.39 & 0.26 \\
\hline & 1.5 & & & & & & & & 1.00 & 0.82 & 0.69 & 0.56 & 0.40 & 0.14 & 0.18 & 0.23 & 0.30 & 0.38 & 0.50 & 0.66 & 0.83 & 0.67 & 0.55 & 0.44 & 0.29 \\
\hline & 1.6 & & & & & & & & & 1.00 & 0.84 & 0.68 & 0.49 & 0.10 & 0.12 & 0.15 & 0.20 & 0.26 & 0.33 & 0.44 & 0.56 & 0.81 & 0.67 & 0.53 & 0.36 \\
\hline & 1.7 & & & & & & & & & & 1.00 & 0.81 & 0.58 & 0.07 & 0.09 & 0.11 & 0.15 & 0.19 & 0.25 & 0.33 & 0.41 & 0.60 & 0.80 & 0.63 & 0.42 \\
\hline & 1.8 & & & & & & & & & & & 1.00 & 0.72 & 0.05 & 0.06 & 0.08 & 0.10 & 0.13 & 0.17 & 0.23 & 0.28 & 0.42 & 0.55 & 0.79 & 0.52 \\
\hline & 1.9 & & & & & & & & & & & & 1.00 & 0.03 & 0.04 & 0.04 & 0.06 & 0.08 & 0.10 & 0.13 & 0.16 & 0.24 & 0.32 & 0.45 & 0.72 \\
\hline \multirow[t]{12}{*}{$\left\langle n_{X}^{4}\right\rangle$} & 0.8 & & & & & & & & & & & & & 1.00 & 0.81 & 0.63 & 0.48 & 0.38 & 0.29 & 0.22 & 0.17 & 0.12 & 0.09 & 0.06 & 0.04 \\
\hline & 0.9 & & & & & & & & & & & & & & 1.00 & 0.78 & 0.60 & 0.47 & 0.36 & 0.27 & 0.22 & 0.15 & 0.11 & 0.08 & 0.05 \\
\hline & 1.0 & & & & & & & & & & & & & & & 1.00 & 0.77 & 0.60 & 0.46 & 0.34 & 0.28 & 0.19 & 0.14 & 0.10 & 0.06 \\
\hline & 1.1 & & & & & & & & & & & & & & & & 1.00 & 0.78 & 0.60 & 0.45 & 0.36 & 0.25 & 0.19 & 0.13 & 0.08 \\
\hline & 1.2 & & & & & & & & & & & & & & & & & 1.00 & 0.76 & 0.57 & 0.46 & 0.31 & 0.24 & 0.17 & 0.10 \\
\hline & 1.3 & & & & & & & & & & & & & & & & & & 1.00 & 0.75 & 0.60 & 0.41 & 0.31 & 0.22 & 0.14 \\
\hline & 1.4 & & & & & & & & & & & & & & & & & & & 1.00 & 0.80 & 0.55 & 0.41 & 0.29 & 0.18 \\
\hline & 1.5 & & & & & & & & & & & & & & & & & & & & 1.00 & 0.68 & 0.52 & 0.36 & 0.23 \\
\hline & 1.6 & & & & & & & & & & & & & & & & & & & & & 1.00 & 0.76 & 0.53 & 0.33 \\
\hline & 1.7 & & & & & & & & & & & & & & & & & & & & & & 1.00 & 0.70 & 0.44 \\
\hline & 1.8 & & & & & & & & & & & & & & & & & & & & & & & 1.00 & 0.63 \\
\hline & 1.9 & & & & & & & & & & & & & & & & & & & & & & & & 1.00 \\
\hline
\end{tabular}

Table B.1: Correlation matrix of the moments $\left\langle n_{X}^{2}\right\rangle$ and $\left\langle n_{X}^{4}\right\rangle$. 


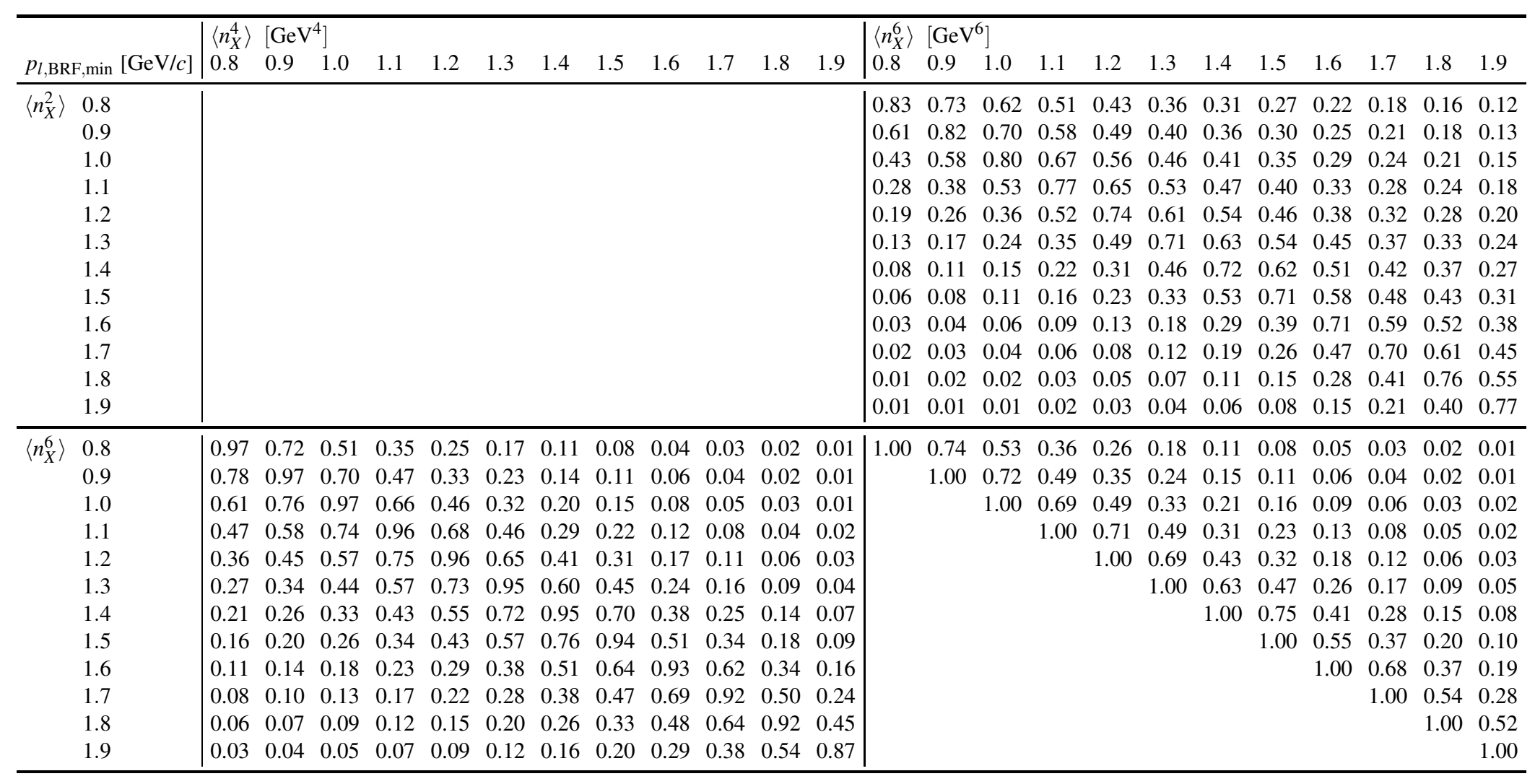

Table B.2: Correlation matrix of the moments $\left\langle n_{X}^{2}\right\rangle,\left\langle n_{X}^{4}\right\rangle$, and $\left\langle n_{X}^{6}\right\rangle$ 


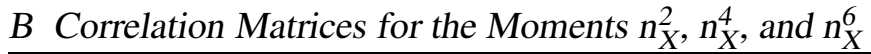




\section{Correlation Matrices for the Mass and Mass-Energy Moments}

The correlation matrices for the moments $\left\langle n_{X}^{k}\right\rangle(k=2,4,6)$ and the moments $\left\langle m_{X}^{l}\right\rangle(l=$ $1, \ldots, 6)$ are presented in this appendix. The matrices use statistical correlations only. The formalism to determine these numbers is reported in section 8.4 . 


\begin{tabular}{|c|c|c|c|c|c|c|c|c|c|c|c|c|c|c|c|c|c|c|c|c|c|c|c|c|c|c|}
\hline \multirow{2}{*}{\multicolumn{2}{|c|}{$p_{l, \mathrm{BRF}, \min }[\mathrm{GeV} / c]$}} & \multicolumn{13}{|c|}{$\left\langle m_{X}^{2}\right\rangle\left[\mathrm{GeV} / c^{2}\right]$} & \multicolumn{12}{|c|}{$\left\langle m_{X}^{4}\right\rangle\left[\mathrm{GeV} / c^{4}\right]$} \\
\hline & & 0.8 & 0.9 & 1. & & 1.1 & 1.2 & 1.3 & 1.4 & 1.5 & 1.6 & 1.7 & 1.8 & 1.9 & 0.8 & 0.9 & 1.0 & 1.1 & 1.2 & 1.3 & 1.4 & 1.5 & 1.6 & 1.7 & 1.8 & 1.9 \\
\hline \multirow[t]{12}{*}{$\left\langle n_{X}^{2}\right\rangle$} & 0.8 & 0.96 & $0.8^{\prime}$ & & & 0.72 & 0.66 & 0.61 & 0.56 & 0.51 & 0.45 & 0.39 & 0.34 & 0.26 & 0.93 & 0.88 & 0.82 & 0.78 & 0.74 & 0.69 & 0.65 & 0.60 & 0.55 & 0.49 & 0.42 & 0.33 \\
\hline & 0.9 & & 0.9 & 0.8 & & 0.79 & 0.74 & 0.67 & 0.62 & 0.56 & 0.50 & 0.44 & 0.37 & 0.29 & 0.83 & 0.93 & 0.88 & 0.83 & 0.79 & 0.74 & 0.69 & 0.64 & 0.58 & 0.52 & 0.45 & 0.36 \\
\hline & 1.0 & 0.74 & 0.8 & 0. & & 0.88 & 0.81 & 0.74 & 0.68 & 0.62 & 0.55 & 0.48 & 0.41 & 0.32 & 0.72 & 0.81 & 0.92 & 0.88 & 0.83 & 0.78 & 0.73 & 0.68 & 0.62 & 0.55 & 0.48 & 0.38 \\
\hline & 1.1 & 0. & 0.7 & 0.8 & & 0.95 & 0.88 & 0.80 & 0.74 & 0.67 & 0.59 & 0.52 & 0.45 & 0.35 & 0.62 & 0.70 & 0.80 & 0.92 & 0.88 & 0.82 & 0.77 & 0.72 & 0.65 & 0.58 & 0.51 & 0.40 \\
\hline & 1.2 & 0. & 0.6 & $0 .{ }^{\prime}$ & & 0.83 & 0.94 & 0.86 & 0.79 & 0.72 & 0.64 & 0.56 & 0.48 & 0.37 & 0.54 & 0.61 & 0.70 & 0.81 & 0.91 & 0.86 & 0.81 & 0.75 & 0.68 & 0.61 & 0.53 & 0.42 \\
\hline & 1.3 & & 0.5 & 0. & & 0.71 & 0.81 & 0.94 & 0.86 & 0.78 & 0.69 & 0.60 & 0.51 & 0.40 & 0.47 & 0.53 & 0.60 & 0.70 & 0.79 & 0.90 & 0.86 & 0.80 & 0.72 & 0.64 & 0.56 & 0.44 \\
\hline & 1.4 & & $0.4^{\prime}$ & 0 & & 0.62 & 0.71 & 0.81 & 0.93 & 0.84 & 0.75 & 0.65 & 0.56 & 0.43 & 0.41 & 0.46 & 0.52 & 0.60 & 0.69 & 0.80 & 0.90 & 0.84 & 0.77 & 0.68 & 0.59 & 0.47 \\
\hline & 1.5 & & 0.4 & 0. & & 0.54 & 0.62 & 0.72 & 82 & 0.92 & 0.82 & 0.71 & 0.61 & 0.47 & 0.36 & 0.40 & 0.46 & 0.53 & 0.61 & 0.70 & 0.80 & 0.89 & 0.82 & 0.73 & 0.63 & 0.50 \\
\hline & 1. & & 0.3 & 0. & & 0.45 & 0.51 & 0.59 & & 0.76 & 0.91 & & & & 0.29 & 0.33 & 0.38 & 0.44 & 0.50 & .58 & 0.66 & 0.74 & 0.88 & 0.79 & 0.69 & 0.55 \\
\hline & 1. & & 0.2 & 0. & & 0.38 & 0. & 0.50 & & 0.64 & 0.76 & & 0. & 60 & 0.25 & 0.28 & 0.32 & 0.37 & 42 & 0.49 & & 0.63 & 0.75 & 0.87 & 0.77 & 0.61 \\
\hline & 1.8 & 21 & 0.2 & 0.2 & & 0.30 & 0.35 & 0.40 & 46 & 0.51 & 0.62 & 0.72 & 0.89 & 0.69 & 0.20 & 0.23 & 0.26 & 0.30 & 0.34 & 0.39 & 0.45 & 0.50 & 0.61 & 0.71 & 0.86 & 0.70 \\
\hline & 1.9 & 0.15 & $0.1^{\prime}$ & 0. & & 0.22 & 0.25 & 0.29 & 0.33 & 0.37 & 0.44 & 0.52 & 0.64 & 0.87 & 0.14 & 0.16 & 0.19 & 0.21 & 0.24 & 0.28 & 0.32 & 0.36 & 0.44 & 0.52 & 0.64 & 0.84 \\
\hline \multirow[t]{12}{*}{$\left\langle n_{X}^{4}\right\rangle$} & 0.8 & .84 & 0.7 & & & 0.62 & 0.58 & 0.53 & 0.49 & 0.44 & 0.39 & 0.34 & 0.29 & 0.23 & 0.96 & 0.90 & 0.85 & 0.80 & 0.76 & 0.71 & 0.67 & 0.62 & 0.56 & 0.50 & 0.44 & 0.34 \\
\hline & 0.9 & & 0.8 & 0. & & 0.69 & 0.64 & 0.59 & 0.54 & 0.49 & 0.43 & 0.38 & 0.32 & 0.25 & 0.78 & 0.96 & 0.90 & 0.85 & 0.81 & 0.76 & 0.71 & 0.66 & 0.60 & 0.53 & 0.46 & 0.37 \\
\hline & 1.0 & & 0.6 & 0.8 & & 0.76 & 0.70 & 0.64 & 0. & 0.53 & 0.47 & 0. & 0.36 & 28 & 0.61 & 0.75 & 0.96 & 0.90 & 0.86 & 0.80 & 0.75 & 0.70 & 0.63 & 0.56 & 0.49 & 0.39 \\
\hline & 1. & & 0.5 & 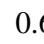 & & 0.82 & 0.76 & 0.69 & 0 & 0.58 & 0.5 & & 0.3 & 0.30 & 0.47 & 0.58 & 0.73 & 0.95 & 0.90 & 0.84 & 0.79 & 0.73 & 0.67 & 0.59 & 0.52 & 0.41 \\
\hline & 1.2 & & 0.3 & 0.6 & & 0.64 & 0.81 & 0.74 & 68 & 0.62 & 0.5 & & 0.41 & 0.32 & 0.37 & 0.45 & 0.57 & 0.74 & 0.94 & 0.88 & 3 & 0.77 & 0.70 & 0.62 & 0.54 & 0.43 \\
\hline & 1.3 & & 0.3 & 0. & & 0.49 & 0.62 & 0.80 & 0.73 & 0.66 & 0.59 & 0. & 0.44 & 0.34 & 0.28 & 0.34 & 0.44 & 0.57 & 0.72 & 0.93 & 8 & 0.81 & 0.74 & 0.65 & 0.57 & 0.45 \\
\hline & 1.4 & & 0.2 & 0.2 & & 0.37 & 0.47 & 0.60 & 0.78 & 0.71 & 0.63 & 0.55 & 0.47 & 0.37 & 0.21 & 0.26 & 0.33 & 0.43 & 0.54 & 0.70 & 0.92 & 0.85 & 0.77 & 0.69 & 0.60 & 0.47 \\
\hline & 1.5 & .15 & 0.1 & 0.2 & & 0.29 & 0.37 & 0.48 & 0.63 & 0.77 & 0.68 & 0.60 & 0.51 & 0.40 & 0.17 & 0.21 & 0.26 & 0.34 & 0.43 & 0.56 & 0.74 & 0.91 & 0.82 & 0.73 & 0.64 & 0.50 \\
\hline & 1.6 & 0.10 & 0.1 & 0. & & 0.20 & 0.25 & 0.33 & 0.43 & 0.53 & 0.75 & 0.66 & 0.56 & 0.44 & 0.11 & 0.14 & 0.18 & 0.23 & 0.30 & 0.38 & 0.50 & 0.62 & 0.89 & 0.79 & 0.69 & 0.54 \\
\hline & 1.7 & 0.08 & 0.0 & 0. & & 0.15 & 0.19 & 0.25 & 0.32 & 0.40 & 0.57 & 0.73 & 0.62 & 0.49 & 0.09 & 0.11 & 0.14 & 0.18 & 0.22 & 0.29 & 0.38 & 0.47 & 0.67 & 0.87 & 0.75 & 0.60 \\
\hline & 1.8 & 0.05 & 0.0 & 0. & & 0.11 & 0.13 & 0.17 & 0.23 & 0.28 & 0.40 & 0.51 & 0.70 & 0.54 & 0.06 & 0.07 & 0.09 & 0.12 & 0.16 & 0.20 & 0.27 & 0.33 & 0.47 & 0.60 & 0.84 & 0.66 \\
\hline & 1.9 & 0.03 & 0.0 & 0. & & 0.07 & 0.08 & 0.11 & 0.14 & 0.17 & 0.25 & 0.32 & 0.44 & 0.66 & 0.04 & 0.05 & 0.06 & 0.08 & 0.10 & 0.13 & 0.17 & 0.20 & 0.29 & 0.38 & 0.52 & 0.80 \\
\hline
\end{tabular}

Table C.1: Correlation matrix of the moments $\left\langle n_{X}^{2}\right\rangle,\left\langle n_{X}^{4}\right\rangle,\left\langle m_{X}^{2}\right\rangle$, and $\left\langle m_{X}^{4}\right\rangle$ 


\section{Translation Formula for Quark Masses from the Kinetic to the $\overline{\mathrm{MS}}$ Scheme}

We convert our results for the quark masses from the kinetic scheme to the $\overline{\mathrm{MS}}$ scheme, using a transformation at order $\alpha_{s}^{2}[32,107]$ as given in the following formula:

$$
\begin{aligned}
m_{q, \overline{\mathrm{MS}}}\left(m_{q}\right) & =m_{\mathrm{kin}}+ \\
& \alpha_{s} \cdot\left(\left(-4 \cdot m_{\mathrm{kin}} \cdot \pi^{-1}\right) / 3 .+\left(16 \cdot \mu \cdot \pi^{-1}\right) / 9 .+\left(2 \cdot m_{\mathrm{kin}}^{-1} \cdot \mu^{2} \cdot \pi^{-1}\right) / 3 .\right)+ \\
& \alpha_{s}^{2} \cdot\left(-\left(m_{\mathrm{kin}} \cdot \log (2.0)\right) / 9 .+\beta_{0} \cdot\left(m_{\mathrm{kin}} \cdot\left(-0.083-\left(71 \cdot \pi^{-2}\right) / 96 .\right)-\right.\right. \\
& \left.\left(m_{\mathrm{kin}} \cdot \log \left(m_{b, \mathrm{kin}}^{2} \cdot m_{\mathrm{kin}}^{-2}\right) \cdot \pi^{-2}\right) / 3 .\right)+ \\
& \mu \cdot\left(\beta _ { 0 } \cdot \left(\left(64 \cdot \pi^{-2}\right) / 27 .+\right.\right. \\
& \left.\left(8 \cdot \log \left(\left(m_{b, \mathrm{kin}} \cdot \mu^{-1}\right) / 2 .\right) \cdot \pi^{-2}\right) / 9 .\right)+ \\
& \left.m_{\mathrm{kin}} \cdot\left(\left(-8 \cdot m_{\mathrm{kin}}^{-1}\right) / 9 .+\left(92 \cdot m_{\mathrm{kin}}^{-1} \cdot \pi^{-2}\right) / 27 .\right)\right)+ \\
& \mu^{2} \cdot\left(m _ { \mathrm { kin } } \cdot \left(-m_{\mathrm{kin}}^{-2} / 3 .+\right.\right. \\
& \left.\left(23 \cdot m_{\mathrm{kin}}^{-2} \cdot \pi^{-2}\right) / 18 .\right)+ \\
& \beta_{0} \cdot\left(\left(13 \cdot m_{\mathrm{kin}}^{-1} \cdot \pi^{-2}\right) / 18 .+\right. \\
& \left.\left.\left(\log \left(\left(m_{b, \mathrm{kin}} \cdot \mu^{-1}\right) / 2 .\right) \cdot m_{\mathrm{kin}}^{-1} \cdot \pi^{-2}\right) / 3 .\right)\right)+ \\
& m_{\mathrm{kin}} \cdot\left(0.583+\left(23 \cdot \pi^{-2}\right) / 72 .+\right. \\
& \left.\left.\left(\pi^{-2} \cdot \zeta(3)\right) / 6 .\right)\right) .
\end{aligned}
$$

The occuring parameters are $\alpha_{s}=0.22, \beta_{0}=9, \mu=1 \mathrm{GeV}$, and $\zeta(3) \sim 1.202$. 



\section{Bibliography}

[1] Csorna, S. E. et al., (CLEO Collaboration), Moments of the B Meson Inclusive Semileptonic Decay Rate Using Neutrino Reconstruction, Phys. Rev. D 70(2004):032002, hep-ex/0403052.

[2] Aubert, B. et al., (BABAR Collaboration), Measurements of Moments of the Hadronic Mass Distribution in Semileptonic B Decays, Phys. Rev. D 69(2004):111103, hep-ex/0403031.

[3] Acosta, D. et al., (CDF Collaboration), Measurement of the Moments of the Hadronic Invariant Mass Distribution in Semileptonic B Decays, Phys. Rev. D 71(2005):051103, hep-ex/0502003.

[4] Abdallah, J. et al., (DELPHI Collaboration), Determination of Heavy Quark NonPerturbative Parameters from Spectral Moments in Semileptonic B Decays, Eur. Phys. J. C 45(2006):35-59, hep-ex/0510024.

[5] Schwanda, C. et al., (Belle Collaboration), Moments of the hadronic invariant mass spectrum in $B \rightarrow$ X/c l nu decays at Belle, Phys. Rev. D 75(2007):032005, hep-ex/0611044.

[6] Gambino, P. and Uraltsev, N., Moments of Semileptonic B Decay Distributions in the 1/mb Expansion, Eur. Phys. J. C 34(2004):181-189, hep-ph/0401063.

[7] Uraltsev, N., Private Communications (2007), Correspondence about HQE Calculations.

[8] Herb, S. W. et al., Observation of a dimuon resonance at $9.5 \mathrm{GeV}$ in 400-GeV proton - nucleus collisions, Phys. Rev. Lett. 39(1977):252-255.

[9] Bebek, C. et al., Evidence for New Flavor Production at the Upsilon (4S), Phys. Rev. Lett. 46(1981):84.

[10] Chadwick, K. et al., (CLEO Collaboration), Decay of b Flavored Hadrons to Single Muon and Dimuon Final States, Phys. Rev. Lett. 46(1981):88-91.

[11] Spencer, L. J. et al., Measurement Of B Meson Semileptonic Decay, Phys. Rev. Lett. 47(1981):771-774.

[12] Fernandez, E. et al., Lifetime of Particles Containing B Quarks, Phys. Rev. Lett. 51(1983):1022.

[13] Lockyer, N. et al., Measurement of the Lifetime of Bottom Hadrons, Phys. Rev. Lett. 51(1983):1316. 
[14] Chau, L.-L. and Keung, W.-Y., Implications from the b-decay measurements, Phys. Rev. D29(1984):592.

[15] Albrecht, H. et al., (ARGUS Collaboration), Measurement of the Decay $B^{0} \rightarrow$ $D^{*-} \ell^{+} v$, Phys. Lett. B197(1987):452.

[16] Albrecht, H. et al., (ARGUS Collaboration), Measurement of $D^{*+}$ Polarization in the Decay $\bar{B}^{0} \rightarrow D^{*+} \ell^{-} \bar{v}$, Phys. Lett. B219(1989):121.

[17] Schröder, H., Proc. XXIV Int. Conf. High Energy Physics (München 1988):79.

[18] Wirbel, M., Stech, B., and Bauer, M., Exclusive Semileptonic Decays of Heavy Mesons, Z. Phys. C29(1985):637.

[19] Körner, J. G. and Schuler, G. A., Exclusive Semileptonic Decays of Bottom Mesons in the Spectator Quark Model, Z. Phys. C38(1988):511.

[20] Körner, J. G. and Schuler, G. A., Aspects of the Chirality of Weak $b \rightarrow c$ Transitions in the Exclusive Semileptonic Decay $B(b) \rightarrow D^{*}(c)$ lepton anti-lepton-neutrino, Phys. Lett. B226(1989):185.

[21] Isgur, N. and Wise, M. B., Weak Decays of Heavy Mesons in the Static Quark Approximation, Phys. Lett. B232(1989):113.

[22] Isgur, N. and Wise, M. B., Weak Transition Form-Factors Between Heavy Mesons, Phys. Lett. B237(1990):527.

[23] Albrecht, H. et al., (ARGUS Collaboration), Investigation of the decays anti-BO $\rightarrow D^{*}+$ lepton- anti-neutrino and anti-B $\rightarrow D^{* *}$ lepton-anti-neutrino, Z. Phys. C57(1993):533-540.

[24] Barberio, E. et al., (Heavy Flavor Averaging Group (HFAG)), Updates of Semileptonic Results for Lepton Photon 2007 (Summer 2007). Available from: http:// www.slac.stanford.edu/xorg/hfag/semi/LP07/home.shtml.

[25] Caprini, I., Lellouch, L., and Neubert, M., Dispersive bounds on the shape of $\bar{B} \rightarrow$ $D^{(*)} \ell \bar{v}$ form factors, Nucl. Phys. B530(1998):153-181, hep-ph/9712417.

[26] Bigi, I. I. Y., Uraltsev, N. G., and Vainshtein, A. I., Nonperturbative corrections to inclusive beauty and charm decays: QCD versus phenomenological models, Phys. Lett. B293(1992):430-436, hep-ph/9207214.

[27] Bigi, I. I., et al., QCD predictions for lepton spectra in inclusive heavy flavor decays, Phys. Rev. Lett. 71(1993)(4):496-499.

[28] Gremm, M. and Kapustin, A., Order $1 / m_{b}^{3}$ corrections to inclusive semileptonic $B$ decay and their implication for the measurement of $\bar{\Lambda}$ and $\lambda_{1}$, Phys. Rev. D55(1997):6924-6932, hep-ph/9603448.

[29] Bauer, C. W. and Trott, M., Reducing theoretical uncertainties in $m_{b}$ and $\lambda_{1}$, Phys. Rev. D67(2003):014021, hep-ph/0205039. 
[30] Cronin-Hennessy, D. et al., (CLEO Collaboration), Hadronic mass moments in inclusive semileptonic B meson decays, Phys. Rev. Lett. 87(2001):251808, hep-ex/0108033.

[31] Mahmood, A. H. et al., (CLEO Collaboration), Measurement of lepton momentum moments in the decay $\bar{B} \rightarrow X \ell \bar{v}$ and determination of heavy quark expansion parameters and $\left|V_{c b}\right|$., Phys. Rev. D67(2003):072001, hep-ex/0212051.

[32] Benson, D., et al., Imprecated, Yet Impeccable: On the Theoretical Evaluation of $\Gamma\left(B \rightarrow X_{c} \ell v\right)$, Nucl. Phys. B665(2003):367-401, hep-ph/0302262.

[33] Aubert, B. et al., (BABAR Collaboration), Measurement of the Electron Energy Spectrum and its Moments in Inclusive $\bar{B} \rightarrow X \ell^{-} v$ Decays, Phys. Rev. D 69(2004):111104, hep-ex/0403030.

[34] Abe, K. et al., (Belle Collaboration), Moments of the electron energy spectrum in $B \rightarrow X_{c} \ell v$ decays at Belle, hep-ex/0508056 (2005), hep-ex/0508056.

[35] Aubert, B. et al., (BABAR Collaboration), Determination of the Branching Fraction for $B \rightarrow X_{c} \ell v$ Decays and of $\left|V_{c b}\right|$ from Hadronic-Mass and Lepton-Energy Moments, Phys. Rev. Lett. 93(2004):011803, hep-ex/0404017.

[36] Buchmüller, O. and Flächer, H., Fit to Moments of Inclusive $B \rightarrow X_{c} \ell v$ and $B \rightarrow X_{s} \gamma$ Decay Distributions using Heavy Quark Expansions in the Kinetic Scheme, Phys. Rev. D 73(2006):073008, hep-ph/0507253.

[37] Glashow, S. L., Partial Symmetries of Weak Interactions, Nucl. Phys. 22(1961):579_ 588.

[38] Weinberg, S., A Model of Leptons, Phys. Rev. Lett. 19(1967)(21):1264-1266.

[39] Salam, A., Weak and Electromagnetic Interactions (1968), Originally printed in *Svartholm: Elementary Particle Theory, Proceedings Of The Nobel Symposium Held 1968 At Lerum, Sweden*, Stockholm 1968, 367-377.

[40] Griffiths, D. J., Introduction to Elementary Particles (John Wiley \& Sons, Inc., New York, 1987), ISBN 3-05-501627-0.

[41] Schmüser, P., Feynman-Graphen und Eichtheorien für Experimentalphysiker (Springer-Verlag, Berlin, Germany / Heidelberg, Germany / London, UK / etc., 1988), ISBN 3-540-18797-9.

[42] Yao, W.-M. et al., (Particle Data Group (PDG)), Review of Particle Physics, Journal of Physics G 33(2006):1. Available from: http://pdg. lbl.gov.

[43] Higgs, P. W., Broken Symmetries, Massless Particles and Gauge Fields, Phys. Lett. 12(1964):132-133.

[44] Higgs, P. W., Broken Symmetries and the Masses of Gauge Bosons, Phys. Rev. Lett. 13(1964):508-509. 
[45] Higgs, P. W., Spontaneous Symmetry Breakdown Without Massless Bosons, Phys. Rev. 145(1966):1156-1163.

[46] Ali, A., B decays, flavour mixings and CP violation in the standard model (1996), hep-ph/9606324.

[47] Schubert, K. R., Quark Mixing and CP Violation (2004), Proceedings contributed to the XXXII International Meeting On Fundamental Physic, Alicante, http://iktp.tu-dresden.de/ schubert/talks/0403-alicante.ps.

[48] Wolfenstein, L., Parametrization of the Kobayashi-Maskawa Matrix, Phys. Rev. Lett. 51(1983): 1945.

[49] Harrison, P. F., e. and Quinn, Helen R., e., (BABAR Collaboration), The BABAR physics book: Physics at an asymmetric B factory, SLAC-R-504 (1998), Papers from Workshop on Physics at an Asymmetric B Factory (BaBar Collaboration Meeting), Rome, Italy, 11-14 Nov 1996, Princeton, NJ, 17-20 Mar 1997, Orsay, France, 16-19 Jun 1997 and Pasadena, CA, 22-24 Sep 1997.

[50] Charles, J. et al., (CKMfitter Group), CP Violation and the CKM Matrix: Assessing the Impact of the Asymmetric B Factories, Eur. Phys. J. C 41(2005):1-131, hep-ph/0406184. Available from: http://ckmfitter.in2p3.fr/.

[51] Manohar, A. V. and Wise, M. B., Heavy Quark Physics (Cambridge University Press, Cambridge, UK, 2000), ISBN 0-521-64241-8.

[52] Roschk, F., Effects of Higher Orders in $1 / m_{b}$ in Semileptonic B-meson Decays, Diplomarbeit, Technische Universität Dresden (2004).

[53] Bigi, I. I. Y., et al., Sum rules for heavy flavor transitions in the SV limit, Phys. Rev. D52(1995):196-235, hep-ph/9405410.

[54] Groom, D. et al., Review of Particle Physics, The European Physical Journal C15(2000):1+. Available from: http://pdg.1bl.gov.

[55] Battaglia, M. et al., The CKM matrix and the unitarity triangle (2003), Based on the workshop held at CERN, 13-16 February 2002, hep-ph/0304132.

[56] Aubert, B. et al., (BABAR Collaboration), Measurement of the inclusive charmless semileptonic branching ratio of $B$ mesons and determination of $\left|V_{u b}\right|$, Phys. Rev. Lett. 92(2004):071802, hep-ex/0307062.

[57] Aubert, B. et al., (BABAR Collaboration), A search for $B^{-} \rightarrow \tau^{-} \bar{v}$ recoiling against a fully reconstructed $B$ (2003), hep-ex/0304030.

[58] Mazur, M. A. and Richman, J., Study of Exclusive Decays $B \rightarrow D / D^{*} \tau \nu$ (2006), BABAR Analysis Document \#1111.

[59] Aubert, B. et al., (BABAR Collaboration), Measurement of the relative branching fractions of $\bar{B} \rightarrow D / D^{*} / D^{* *} \ell^{-} \bar{v}$ decays in events with a fully reconstructed $B$ meson (2007), hep-ex/0703027. 
[60] Sundermann, J. E., Improved Measurements of Hadronic Mass Moments in Decays $B \rightarrow X_{c} \ell v$ and Determination of $\left|V_{c b}\right|$, Dissertation, Technische Universität Dresden (2006).

[61] Aubert, B. et al., (BABAR Collaboration), Measurement of Moments of the HadronicMass and-Energy Spectrum in Inclusive Semileptonic $\bar{B} \rightarrow X_{c} \ell^{-} \bar{v}$ Decays (2007), arXiv:0707.2670 [hep-ex].

[62] PEP-II: An Asymmetric B Factory. Conceptual Design Report, SLAC-R-418 (1993).

[63] Aubert, B. et al., (BABAR Collaboration), The BaBar Detector, Nucl. Instrum. Meth. A 479(2002):1-116, hep-ex/0105044.

[64] Santonico, R. and Cardarelli, R., Development of Resistive Plate Counters, Nucl. Instrum. Meth. A 187(1981):377-380.

[65] BABAR Collaboration, Inventory of PidSelectors for r14a (2004). Available from: http://www.slac.stanford.edu/BFR0OT/www/Physics/Tools/Pid/ Selectors/r14a/selectors.html.

[66] Benelli, G., et al., The BABAR LST Detector High Voltage System: Design and Implementation, IEEE Nucl. Sci. Symp. Conf. Rec. 2(2006):1145-1148.

[67] Hearty, C., Measurement of the Number of $\Upsilon(4 S)$ Mesons Produced in Run 1 (B Counting) (2001), BABAR Analysis Document \#134.

[68] Lange, D. J., The EvtGen Particle Decay Simulation Package, Nucl. Instrum. Meth. A 462(2001):152-155.

[69] Agostinelli, S. et al., (GEANT4 Collaboration), GEANT4: A Simulation Toolkit, Nucl. Instrum. Meth. A 506(2003):250-303.

[70] Richter-Was, E., QED Bremsstrahlung In Semileptonic B and Leptonic $\tau$ Decays, Phys. Lett. B 303(1993):163-169.

[71] Lopes Pegna, D. and Lüth, V., (Semileptonic Analysis Working Group (BABAR)), $B \rightarrow X_{c} \ell v$ Reweighting 2007 (August 2007), Corrections made on the second url. Available from: http://www.slac.stanford.edu/BFROOT/www/Physics/ Analysis/AWG/Semileptonic/Reweighting2007.html, http://babar-hn . slac.stanford.edu:5090/HyperNews/get/semi_lept_decays/779.html.

[72] del Re, D., Semi-Exclusive B reconstruction (2001), BABAR Analysis Document \#271.

[73] Smith, J., Soffer, A., and Waldi, R., Recommendation for Exclusive B Reconstruction Analysis Variables (June 1991). Available from: http://www.slac. stanford.edu/BFROOT/www/doc/public/babar_notes/index.html. 
[74] Ulmer, K., Correcting COM Beam Energies (November 24, 2004), BABAR Collaboration, Presented at Wednesday Physics Meeting. Available from: http://www. slac.stanford.edu/BFRO0T/www/Physics/WednesdayMeetings/W112404/ Ulmer.pdf.

[75] Ulmer, K. (March 29, 2005), BABAR Hypernews Forum for Conditions Database Management. Available from: http://babar-hn.slac.stanford.edu:5090/ HyperNews/get/condmgmt/81/2/1.html.

[76] Fortin, D. et al., Particle Selection Criteria, Optimized for Visible Energy Measurement (2003), BABAR Analysis Document \#633.

[77] Drescher, A. et al., The Argus Electron - Photon Calorimeter. 3. Electron - Hadron Separation, Nucl. Instrum. Meth. A237(1985):464-474.

[78] Brandt, T., Likelihood Based Electron Identification (2002), BABAR Analysis Document \#396.

[79] Band, H. et al., Studies of A Neural Net Based Muon Selector for the BABAR Experiment (2004), BABAR Analysis Document \#474.

[80] Mancinelli, G. and Spanier, S., Kaon Selection at the BABAR Experiment (2001), BABAR Analysis Document \#116.

[81] BABAR Collaboration, Performing Particle ID on Monte Carlo (2006). Available from: http://www.slac.stanford.edu/BFROOT/www/Physics/Tools/ Pid/PidOnMc/pidonmc.html.

[82] Kowalewski, R. V., Mmiss2 versus Emiss-Pmiss; why it matters (February 2003), Posted in the BABAR Hypernews for Inclusive Semi-Leptonic Decays. Available from: http://babar-hn.slac.stanford.edu:5090/HyperNews/get/ ISLDecays/288.html.

[83] Blobel, V. and Lohrmann, E., Statistische und numerische Methoden der Datenanalyse (Teubner, 1998), ISBN 3519032430.

[84] Klose, V. and Sundermann, J. E., KinFitter - A Kinematic Fit with Constraints (2004), BABAR Analysis Document \#1061.

[85] Albrecht, H. et al., (ARGUS Collaboration), Reconstruction Of B Mesons, Phys. Lett. B 185(1987):218.

[86] Skwarnicki, T., (Crystal Ball Collaboration), A Study Of The Radiative Cascade Transitions Between The Yı And $\Upsilon$ Resonances, DESY F31-86-02 (1986).

[87] Aubert, B. et al., (BABAR Collaboration), Study of inclusive $B^{-}$and $\bar{B}^{0}$ decays to flavor-tagged $D, D_{s}$ and $\Lambda_{c}^{+}$, Phys. Rev. D75(2007):072002, hep-ex/0606026.

[88] Adam, N. E. et al., (CLEO Collaboration), Absolute branching fraction measurements for $D^{+}$and $D^{0}$ inclusive semileptonic decays, Phys. Rev. Lett. 97(2006):251801, hep-ex/0604044. 
[89] Albrecht, H. et al., (ARGUS Collaboration), A Model-Independent Determination of the Inclusive Semileptonic Decay Fraction of B Mesons, Phys. Lett. B 318(1993):397-404.

[90] Matyja, A. et al., (Belle Collaboration), Observation of $B^{0} \rightarrow D^{*-} \tau^{+} v_{\tau}$ decay at Belle (2007), arXiv:0706.4429 [hep-ex], submitted to Phys. Rev. Lett.

[91] Aubert, B. et al., (BABAR Collaboration), Observation of the Semileptonic Decays $B \rightarrow D^{*} \tau \bar{v}$ and Evidence for $B \rightarrow D \tau \bar{v}$ (2007), arXiv:0709.1698 [hep-ex], submitted to Phys. Rev. Lett.

[92] Barberio, E. et al., (Heavy Flavor Averaging Group (HFAG)), Updates of Semileptonic Results for Summer 2006 (Summer 2006). Available from: http://www . slac.stanford.edu/xorg/hfag/semi/summer06/home.shtml.

[93] Yao, W.-M. et al., (Particle Data Group (PDG)), Particle Listings in the 2007 Review of Particle Physics (partial update) (2007). Available from: http://pdg. Ibl .gov.

[94] Barberio, E. et al., (Heavy Flavor Averaging Group (HFAG)), Results for the PDG 2007 web update (March 2007). Available from: http://www.slac.stanford. edu/xorg/hfag/osc/PDG_2007/.

[95] Lange, B. O., Neubert, M., and Paz, G., Theory of Charmless Inclusive B Decays and the Extraction of $V_{u b}$, Phys. Rev. D 72(2005):073006, hep-ph/0504071.

[96] Klose, V., A Measurement of the $\pi^{0}$ Efficiency using $D^{0}$ decays into $K \pi \pi^{0}$ (2006), BABAR Analysis Document \#1194.

[97] Räder, C., Kobel, M., and Lacker, H., Determination of the Reconstruction Efficiency of Low Energy $\pi^{0}$ Mesons at the BABAR Experiment (2007), BABAR Analysis Document \#1823.

[98] Allen, M. et al., A Measurement of $\pi^{0}$ Efficiency Using $\tau \rightarrow \rho v$ and $\tau \rightarrow \pi \nu$ Decays (2004), BABAR Analysis Document \#870.

[99] Dingfelder, J. (July 13, 2007), BABAR Hypernews Forum for Review of SemiLep-03/02. Available from: http://babar-hn.slac.stanford.edu: 5090/HyperNews/get/rev-SemiLep-03-02/30/1.html.

[100] Benson, D., Bigi, I. I., and Uraltsev, N., On the Photon Energy Moments and their 'Bias' Corrections in $B \rightarrow X_{s} \gamma$, Nucl. Phys. B710(2005):371-401, hep-ph/0410080.

[101] Aquila, V., et al., Perturbative Corrections to Semileptonic b Decay Distributions, Nucl. Phys. B719(2005):77-102, hep-ph/0503083.

[102] Uraltsev, N., Perturbative Corrections to the Semileptonic b-decay Moments: $E_{c u t}^{\ell}$ Dependence and Running- $\alpha_{s}$ Effects in the OPE Approach, Int. J. Mod. Phys. A 20(2005):2099-2118, hep-ph/0403166.

[103] Bigi, I. I., Uraltsev, N., and Zwicky, R., On the nonperturbative charm effects in inclusive $B \rightarrow X_{c} \ell v$ decays, Eur. Phys. J. C 50(2007):539-556, hep-ph/0511158. 
[104] Bigi, I., et al., High power $n$ of $m_{b}$ in b-flavored widths and $n=5 \rightarrow \infty$ limit, Phys. Rev. D 56(1997)(7):4017-4030.

[105] Aubert, B. et al., (BABAR Collaboration), Measurements of the $B \rightarrow X_{s} \gamma$ Branching Fraction and Photon Spectrum from a Sum of Exclusive Final States, Phys. Rev. D 72(2005):052004, hep-ex/0508004.

[106] Aubert, B. et al., (BABAR Collaboration), Measurement of the branching fraction and photon energy moments of $B \rightarrow X_{s} \gamma$ and $A_{C P}\left(B \rightarrow X_{(s+d)} \gamma\right)$, Phys. Rev. Lett. 97(2006):171803, hep-ex/0607071.

[107] Gambino, P. and Giordano, P., Private Communications (2007), Correspondence about new HQE Calculations.

[108] Bauer, C. W. et al., Global Analysis of Inclusive B Decays, Phys. Rev. D 70(2004):094017, hep-ph/0408002.

[109] Hashimoto, S., et al., Lattice calculation of the zero-recoil form factor of $B \rightarrow D^{*} \ell v$ Toward a model independent determination of $\left|V_{c b}\right|$, Phys. Rev. D 66(2002)(1):014503.

[110] Tackmann, K., Determination of the b-quark mass and other non-perturbative parameters in semileptonic and radiative penguin decays at BABAR, Presented at HADRON07. Available from: https://agenda.infn.it/materialDisplay. py?contribId=73\&ampsessionId=15\&amp; materialId=slides\&amp; conf $I d=52$.

[111] Abe, K. et al., (Belle Collaboration), Determination of $\left|V_{c b}\right|$ and $m_{b}$ from inclusive $B \rightarrow X_{c} \ell v$ and $B \rightarrow X_{s} \gamma$ decays at Belle (2006), hep-ex/0611047.

[112] Schwanda, C., Private Communications (2007), Correspondence about the Belle HQE Fit. 


\section{Danksagung}

Am Ende dieser Arbeit möchte ich all denjenigen danken, die direkt und indirekt daran beteiligt waren.

Mein besonderer Dank gilt Herrn Schubert, der durch hervorragende Betreuung und interessante Diskussionen diese Arbeit begleitet hat. Die Möglichkeit, in der BABAR-Gruppe mitzuarbeiten und viele meiner eigenen Ideen zu verfolgen, hat mir viel bedeutet.

Heiko Lacker möchte ich ebenso herzlich dafür danken, dass er selbst unter großer Arbeitsbelastung immer ein offenes Ohr für Fragen hatte und sie vor allem auch immer beantworten konnte. Mit unermüdlichem Einsatz hat er in unserem Gruppenseminar für Teilchenphysik-Gehirnjogging gesorgt. Besonders freue ich mich, dass er bereit ist, als Gutachter dieser Dissertation zu fungieren.

Herrn Mannel bin ich sehr dankbar, dass er bereit ist, diese Arbeit zu begutachten, und ich freue mich auf folgende Diskussionen über HQE-Fits.

Michael Kobel danke ich für sein Engagement, die BABAR-Arbeitsgruppe in Dresden weiterzuführen und uns vollstens zu unterstützen.

Die ganze Arbeit wäre nicht möglich gewesen ohne die aufopfernde Arbeit von Rainer Schwierz und Andreas Petzold für unser Rechnersystem. Sätze wie „... aber das ist doch überhaupt kein Problem... “ oder ,... das kriegen wir schon hin...", sind bei euch keine Seltenheit, dafür mein Riesen-Dank.

Genauso wäre diese fulminante Zeit kaum vergleichbar gewesen ohne bedeutungsschwangere Diskussionen mit dem Rest der Belegschaft des IKTP, allen voran Denis Altenburg, Thomas Göpfert, Petzi, und Jan Erik Sundermann. Wir sind alle Meister in der Benutzung von Fachjargon und Experten auf dem Gebiet der corporate Problemlösung. Florian Bernlochner danke ich für mühevolles Korrekturlesen sowie großartige Kohlrouladen.

Meiner Basketballmannschaft sowie allen Mini-Basketballern in Dresden danke ich für die Mithilfe bei der Weiterentwicklung meiner Soft-Skills sowie für den nötigen Ausgleich. Ganz besonderer, persönlicher Dank geht nochmals an Thomas. 



\section{Versicherung}

Hiermit versichere ich, dass ich die vorliegende Arbeit ohne unzulässige Hilfe Dritter und ohne Benutzung anderer als der angegebenen Hilfsmittel angefertigt habe; die aus fremden Quellen direkt oder indirekt übernommenen Gedanken sind als solche kenntlich gemacht. Die Arbeit wurde bisher weder im Inland noch im Ausland in gleicher oder ähnlicher Form einer anderen Prüfungsbehörde vorgelegt.

Diese Arbeit wurde am Institut für Kern- und Teilchenphysik der Technischen Universität Dresden unter wissenschaftlicher Betreuung von Prof. Dr. Klaus R. Schubert angefertigt.

Es haben keine früheren erfolglosen Promotionsverfahren stattgefunden.

Ich erkenne die Promotionsordnung der Fakultät Mathematik und Naturwissenschaften an der Technischen Universität Dresden vom 20. März 2000, in der Fassung der vom Fakultätsrat am 19.06.2002 und 12.07.2002 beschlossenen und mit Erlass des Sächsischen Staatsministeriums für Wissenschaft und Kunst vom 18.03.2003 genehmigten Änderungen gemäß Satzung vom 16.04.2003, an.

Dresden, den 29.11.2007

Verena Klose 